\title{
Electron-Ion Collider: The next QCD frontier
}

\section{Understanding the glue that binds us all}

A. Accardi ${ }^{14,28}$, J.L. Albacete ${ }^{16}$, M. Anselmino ${ }^{29}$, N. Armesto ${ }^{37}$, E.C. Aschenauer ${ }^{3, a}$, A. Bacchetta ${ }^{36}$, D. Boer $^{33}$, W.K. Brooks ${ }^{38, a}$, T. Burton ${ }^{3}$, N.-B. Chang ${ }^{23}$, W.-T. Deng ${ }^{13,23}$, A. Deshpande ${ }^{25, a, b, c}$, M. Diehl ${ }^{11, a}$, A. Dumitru ${ }^{2}$, R. Dupré ${ }^{2}$, R. Ent ${ }^{28, d}$, S. Fazio ${ }^{3}$, H. Gao ${ }^{12, a}$, V. Guzey ${ }^{28}$, H. Hakobyan ${ }^{38}$, Y. Hao ${ }^{3}$, D. Hasch ${ }^{15}$, R. Holt ${ }^{1, a}$,

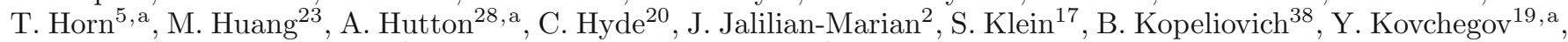

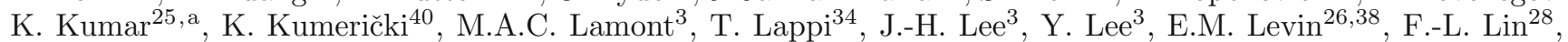
V. Litvinenko ${ }^{3}$, T.W. Ludlam ${ }^{3, d}$, C. Marquet ${ }^{8}$, Z.-E. Meziani ${ }^{27, a, b, e}$, R. McKeown ${ }^{28, d}$, A. Metz ${ }^{27}$, R. Milner ${ }^{18}$, V.S. Morozov ${ }^{28}$, A.H. Mueller ${ }^{9, a}$, B. Müller ${ }^{3,12, d}$, D. Müller ${ }^{22}$, P. Nadel-Turonski ${ }^{28}$, H. Paukkunen $^{34}$, A. Prokudin $^{28}$,

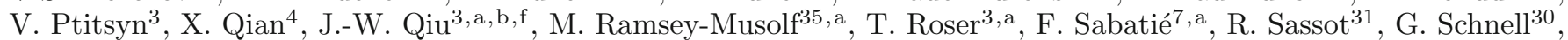
P. Schweitzer ${ }^{32}$, E. Sichtermann ${ }^{17, a}$, M. Stratmann ${ }^{39}$, M. Strikman ${ }^{21}$, M. Sullivan ${ }^{24}$, S. Taneja ${ }^{10,25}$, T. Toll ${ }^{3}$,

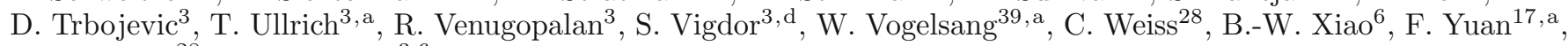
Y.-H. Zhang ${ }^{28}$, and L. Zheng 3,6

1 Argonne National Laboratory, Argonne, IL, USA

2 Baruch College, CUNY, New York, NY, USA

3 Brookhaven National Laboratory, Upton, NY, USA

4 California Institute of Technology, Pasadena, CA, USA

5 The Catholic University of America, N.E. Washington, DC, USA

${ }^{6}$ Central China Normal University, Wuhan, Hubei, China

7 CEA, Centre de Saclay, Gif-sur-Yvette, France

8 CERN, Geneva, Switzerland

9 Columbia University, New York, NY, USA

10 Dalhousie University, Halifax, Nova Scotia, Canada

11 DESY, Hamburg, Germany

${ }^{12}$ Duke University, Durham, NC, USA

13 Frankfurt University, FIAS, Frankfurt, Germany

${ }^{14}$ Hampton University, Hampton, VA, USA

15 INFN-LNF, Frascati, Italy

16 IPNO, Université Paris-Sud 11, CNRS/IN2P3, Orsay, France

17 Lawrence Berkeley National Laboratory, Berkeley, CA, USA

18 Massachusetts Institute of Technology, Cambridge, MA, USA

19 The Ohio State University, Columbus, OH, USA

${ }^{20}$ Old Dominion University, Norfolk, VA, USA

21 Pennsylvania State University, Philadelphia, PA, USA

${ }^{22}$ Ruhr-University Bochum, Bochum, Germany

23 Shandong University, Shandong, China

24 Stanford Linear Accelerator Center, Menlo Park, CA, USA

25 Stony Brook University, Stony Brook, NY, USA

26 Tel Aviv University, Tel Aviv, Israel

27 Temple University, Philadelphia, PA, USA

28 Thomas Jefferson National Accelerator Facility, Newport News, VA, USA

${ }^{29}$ Torino University \& INFN, Torino, Italy

30 University of Basque Country, Bilbao, Spain

31 University of Buenos Aires, Buenos Aires, Argentina

${ }^{32}$ University of Connecticut, Storrs, CT, USA

33 University of Groningen, Groningen, The Netherlands

34 University of Jyvaskyla, Jyvaskyla, Finland

35 University of Massachusetts at Amherst, Amherst, MA, USA

36 University of Pavia, Pavia, Italy

37 University of Santiago de Campostela, Santiago de Compostela, Spain

38 Universidad Técnica Federico Santa Maria, Valparaíso, Chile

39 University of Tübingen, Tübingen, Germany

40 University of Zagreb, Zagreb, Croatia 
Received: 21 March 2016

Published online: 8 September 2016

(C) The Author(s) 2016. This article is published with open access at Springerlink.com Communicated by N. Alamanos

\begin{abstract}
This White Paper presents the science case of an Electron-Ion Collider (EIC), focused on the structure and interactions of gluon-dominated matter, with the intent to articulate it to the broader nuclear science community. It was commissioned by the managements of Brookhaven National Laboratory (BNL) and Thomas Jefferson National Accelerator Facility (JLab) with the objective of presenting a summary of scientific opportunities and goals of the EIC as a follow-up to the 2007 NSAC Long Range plan. This document is a culmination of a community-wide effort in nuclear science following a series of workshops on EIC physics over the past decades and, in particular, the focused ten-week program on "Gluons and quark sea at high energies" at the Institute for Nuclear Theory in Fall 2010. It contains a brief description of a few golden physics measurements along with accelerator and detector concepts required to achieve them. It has been benefited profoundly from inputs by the users' communities of BNL and JLab. This White Paper offers the promise to propel the QCD science program in the US, established with the CEBAF accelerator at JLab and the RHIC collider at BNL, to the next QCD frontier.
\end{abstract}

\section{Preamble}

\section{Editors' note for the second edition}

The first edition of this White Paper was released in 2012. In the current (second) edition, the science case for the EIC is further sharpened in view of the recent data from BNL, CERN and JLab experiments and the lessons learnt from them. Additional improvements were made by taking into account suggestions from the larger nuclear physics community including those made at the EIC Users Group meeting at Stony Brook University in July 2014, and the QCD Town Meeting at Temple University in September 2014.

Abhay Deshpande, Zein-Eddine Meziani and Jian-Wei Qiu

November 2014

\section{Editors' note for the third edition}

Since the 2nd release of this White Paper, the NSAC's Long Range Plan (2015) was successfully completed. The $\mathrm{EIC}$ is a major recommendation of the US nuclear science community. In the current release (version 3) we have fixed some minor remaining errors in the text, and have added a few new references. While the core science case for the EIC remains the same, the machine designs of both options, the eRHIC at BNL and the JLEIC at JLab keep evolving. In this 3rd release of the EIC White Paper instead of making substantial changes to the machine design sections (5.1 and 5.2), we give references to the most recent machine design documents.

$$
\begin{array}{r}
\text { Abhay Deshpande, Zein-Eddine Meziani } \\
\text { and Jian-Wei Qiu }
\end{array}
$$
June 2016

\section{Contents}

Executive summary: exploring the glue that binds us all 3

1 Overview: science, machine and deliverables of the EIC 4

1.1 Scientific highlights . . . . . . . . . . . . . . 4

1.2 The EIC and its realization . . . . . . . . . 7

1.3 Physics deliverables of the EIC . . . . . . . 8

2 Spin and three-dimensional structure of the nucleon 9

2.1 Introduction . . . . . . . . . . . . . . 9

2.2 The longitudinal spin of the nucleon . . . . . . 13

2.3 Confined motion of partons in nucleons: TMDs 20

2.4 Spatial imaging of quarks and gluons . . . . 26

3 The nucleus: a laboratory for QCD . . . . . . . . 34

3.1 Introduction . . . . . . . . . . . . . . . . 34

3.2 Physics of high gluon densities in nuclei . . . 38

3.3 Quarks and gluons in the nucleus ..... 56

3.4 Connections to $p+A, \mathrm{~A}+\mathrm{A}$ and cosmic ray physics .................. 62

4 Possibilities at the luminosity frontier: physics beyond the Standard Model . . . . . . . . . . . . . . 71

4.1 Introduction . . . . . . . . . . . . . . . . 71

4.2 Specific opportunities in electroweak physics . . 72

4.3 EIC requirements for electroweak physics measurements . . . . . . . . . . . . . . . 74

5 The accelerator designs and challenges . . . . . . 74

5.1 eRHIC . . . . . . . . . . . . . . . . . . . . . . . . . . . .

5.2 JLEIC . . . . . . . . . . . . . . . . 78

6 The EIC detector requirements and design ideas . 83

6.1 Introduction . . . . . . . . . . . . . . . . . . 83

6.2 Kinematic coverage . . . . . . . . . . . . 83

6.3 Recoil baryon angles and $t$ resolution . . . . . . 87

6.4 Detector and interaction region (IR) layout . . 88

\footnotetext{
${ }^{a}$ Writing Committee Members.

b Editors.

c e-mail: abhay.deshpande@stonybrook.edu

d Laboratory Management Representatives.

e e-mail: meziani@temple.edu

f e-mail: jqiu@bnl.gov
} 


\section{Executive summary: exploring the glue that binds us all}

Nuclear science is concerned with the origin and structure of the core of the atom, the nucleus and the nucleons (protons and neutrons) within it, which account for essentially all of the mass of the visible universe. Half a century of investigations have revealed that nucleons are themselves composed of more basic constituents called quarks, bound together by the exchange of gluons, and have led to the development of the fundamental theory of strong interactions known as Quantum Chromo-Dynamics (QCD). Understanding these constituent interactions and the emergence of nucleons and nuclei from the properties and dynamics of quarks and gluons in QCD is a fundamental and compelling goal of nuclear science.

QCD attributes the forces among quarks and gluons to their "color charge". In contrast to the quantum electromagnetism, where the force carrying photons are electrically neutral, gluons carry color charge. This causes the gluons to interact with each other, generating a significant fraction of the nucleon mass and leading to a littleexplored regime of matter, where abundant gluons dominate its behavior. Hints of this regime become manifest when nucleons or nuclei collide at nearly the speed of light, as they do in colliders such as HERA, RHIC and the LHC. The quantitative study of matter in this new regime requires a new experimental facility: an Electron-Ion Collider (EIC).

In the last decade, nuclear physicists have developed new phenomenological tools to enable remarkable tomographic images of the quarks and gluons inside unpolarized as well as polarized protons and neutrons. These tools will be further developed and utilized to study predominantly the valence quarks in the nucleon at the upgraded $12 \mathrm{GeV}$ CEBAF at JLab and COMPASS at CERN. Applying these new tools to study the matter dominated by gluons and sea quarks originating from gluons will require the higher energy and beam polarization of an EIC.

As one increases the energy of the electron-nucleon collision, the process probes regions of progressively higher gluon density. However, the density of gluons inside a nucleon must eventually saturate to avoid untamed growth in the strength of the nucleon-nucleon interaction, which would violate the fundamental principle of unitarity. To date this saturated gluon density regime has not been clearly observed, but an EIC could enable detailed study of this remarkable aspect of matter. This pursuit will be facilitated by electron collisions with heavy nuclei, where coherent contributions from many nucleons effectively amplify the gluon density being probed.

The EIC was designated in the 2007 Nuclear Physics Long Range Plan as "embodying the vision for reaching the next QCD frontier" [1]. It would extend the QCD science programs in the US established at both the CEBAF accelerator at JLab and RHIC at BNL in dramatic and fundamentally important ways. The most intellectually pressing questions that an EIC will address that relate to our detailed and fundamental understanding of QCD in this frontier environment are:

- How are the sea quarks and gluons, and their spins, distributed in space and momentum inside the nucleon? How are these quark and gluon distributions correlated with overall nucleon properties, such as spin direction? What is the role of the orbital motion of sea quarks and gluons in building the nucleon spin?

- Where does the saturation of gluon densities set in? Is there a simple boundary that separates this region from that of more dilute quark-gluon matter? If so, how do the distributions of quarks and gluons change as one crosses the boundary? Does this saturation produce matter of universal properties in the nucleon and all nuclei viewed at nearly the speed of light?

- How does the nuclear environment affect the distribution of quarks and gluons and their interactions in nuclei? How does the transverse spatial distribution of gluons compare to that in the nucleon? How does nuclear matter respond to a fast moving color charge passing through it? Is this response different for light and heavy quarks?

Answers to these questions are essential for understanding the nature of visible matter. An EIC is the ultimate machine to provide answers to these questions for the following reasons:

- a collider is needed to provide kinematic reach well into the gluon-dominated regime;

- electron beams are needed to bring to bear the unmatched precision of the electromagnetic interaction as a probe;

- polarized nucleon beams are needed to determine the correlations of sea quark and gluon distributions with the nucleon spin;

- heavy-ion beams are needed to provide precocious access to the regime of saturated gluon densities and offer a precise dial in the study of propagation length for color charges in nuclear matter.

The EIC would be distinguished from all past, current, and contemplated facilities around the world by being at the intensity frontier with a versatile range of kinematics and beam polarizations, as well as beam species, allowing the above questions to be tackled at one facility. In particular, the EIC design exceeds the capabilities of HERA, the only electron-proton collider to date, by adding a) polarized proton and light-ion beams; b) a wide variety of heavy-ion beams; c) two to three orders of magnitude increase in luminosity to facilitate tomographic imaging; and d) wide energy variability to enhance the sensitivity to gluon distributions. Achieving these challenging technical improvements in a single facility will extend US leadership in accelerator science and in nuclear science.

The scientific goals and the machine parameters of the EIC were delineated in deliberations at a communitywide program held at the Institute for Nuclear Theory 


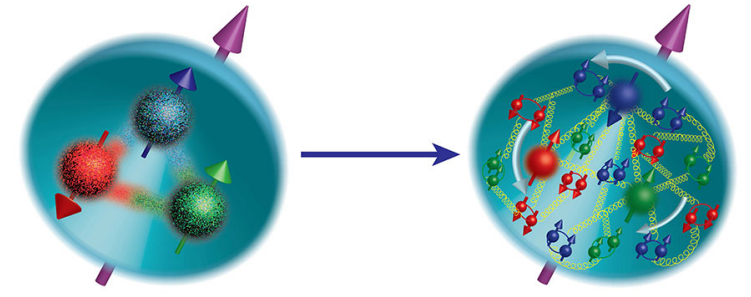

Fig. 1. Evolution of our understanding of nucleon spin structure. Left: in the 1980s, a nucleon's spin was naively explained by the alignment of the spins of its constituent quarks. Right: in the current picture, valence quarks, sea quarks and gluons, and their possible orbital motion are expected to contribute to the overall nucleon spin.

(INT) [2]. The physics goals were set by identifying critical questions in QCD that remain unanswered despite the significant experimental and theoretical progress made over the past decade. This White Paper is prepared for the broader nuclear science community, and presents a summary of those scientific goals with a brief description of the golden measurements and accelerator and detector technology advances required to achieve them.

\section{Overview: science, machine and deliverables of the EIC}

\subsection{Scientific highlights}

\subsubsection{Nucleon spin and its 3D structure and tomography}

Several decades of experiments on deep inelastic scattering (DIS) of electron or muon beams off nucleons have taught us about how quarks and gluons (collectively called partons) share the momentum of a fast-moving nucleon. They have not, however, resolved the question of how partons share the nucleon's spin and build up other nucleon intrinsic properties, such as its mass and magnetic moment. The earlier studies were limited to providing the longitudinal-momentum distribution of quarks and gluons, a one-dimensional view of nucleon structure. The EIC is designed to yield much greater insight into the nucleon structure (fig. 1, from left to right), by facilitating multidimensional maps of the distributions of partons in space, momentum (including momentum components transverse to the nucleon momentum), spin, and flavor.

The $12 \mathrm{GeV}$ upgrade of CEBAF at JLab and the COMPASS at CERN will initiate such studies in predominantly valence quark region. However, these programs will be dramatically extended at the EIC to explore the role of the gluons and sea quarks in determining the hadron structure and properties. This will resolve crucial questions, such as whether a substantial "missing" portion of nucleon spin resides in the gluons. By providing highenergy probes of partons' transverse momenta, the EIC should also illuminate the role of their orbital motion contributing to nucleon spin.

\section{The spin and flavor structure of the nucleon}

An intensive and worldwide experimental program over the past two decades has shown that the spin of quarks and antiquarks is only responsible for $\sim 30 \%$ of the proton spin. Recent RHIC results indicate that the gluons' spin contribution in the currently explored kinematic region is non-zero, but not yet sufficient to account for the missing $70 \%$. The partons' total helicity contribution to the proton spin is very sensitive to their minimum momentum fraction $x$ accessible by the experiments. With the unique capability to reach two orders of magnitude lower in $x$ and to span a wider range of momentum transfer $Q$ than previously achieved, the EIC would offer the most powerful tool to precisely quantify how the spin of gluons and that of quarks of various flavors contribute to the protons spin. The EIC would realize this by colliding longitudinally polarized electrons and nucleons, with both inclusive and semi-inclusive DIS measurements. In the former, only the scattered electron is detected, while in the latter, an additional hadron created in the collisions is to be detected and identified.

Figure 13 in sect. 2.2 shows the reduction in uncertainties of the contributions to the nucleon spin from the spin of the gluons, quarks and antiquarks, evaluated in the $x$ range from 0.001 to 1.0. This would be achieved by the EIC in its early operations. In future, the kinematic range could be further extended down to $x \sim 0.0001$ reducing significantly the uncertainty on the contributions from the unmeasured small- $x$ region. While the central values of the helicity contributions in fig. 13 are derived from existing data, they could change as new data become available in the low- $x$ region. The uncertainties calculated here are based on the state-of-the art theoretical treatment of all available data related to the nucleon spin puzzle. Clearly, the EIC will make a huge impact on our knowledge of these quantities, unmatched by any other existing or anticipated facility. The reduced uncertainties would definitively resolve the question of whether parton spin preferences alone can account for the overall proton spin, or whether additional contributions are needed from the orbital angular momentum of partons in the nucleon.

\section{The confined motion of partons inside the nucleon}

Semi-inclusive DIS (SIDIS) measurements have two natural momentum scales: the large momentum transfer from the electron beam needed to achieve the desired spatial resolution, and the momentum of the produced hadrons perpendicular to the direction of the momentum transfer, which prefers a small value sensitive to the motion of confined partons. Remarkable theoretical advances over the past decade have led to a rigorous framework where information on the confined motion of the partons inside a fast-moving nucleon is matched to transversemomentum-dependent parton distributions (TMDs). In particular, TMDs are sensitive to correlations between the motion of partons and their spin, as well as the spin of the parent nucleon. These correlations can arise from spin-orbit coupling among the partons, about which very 


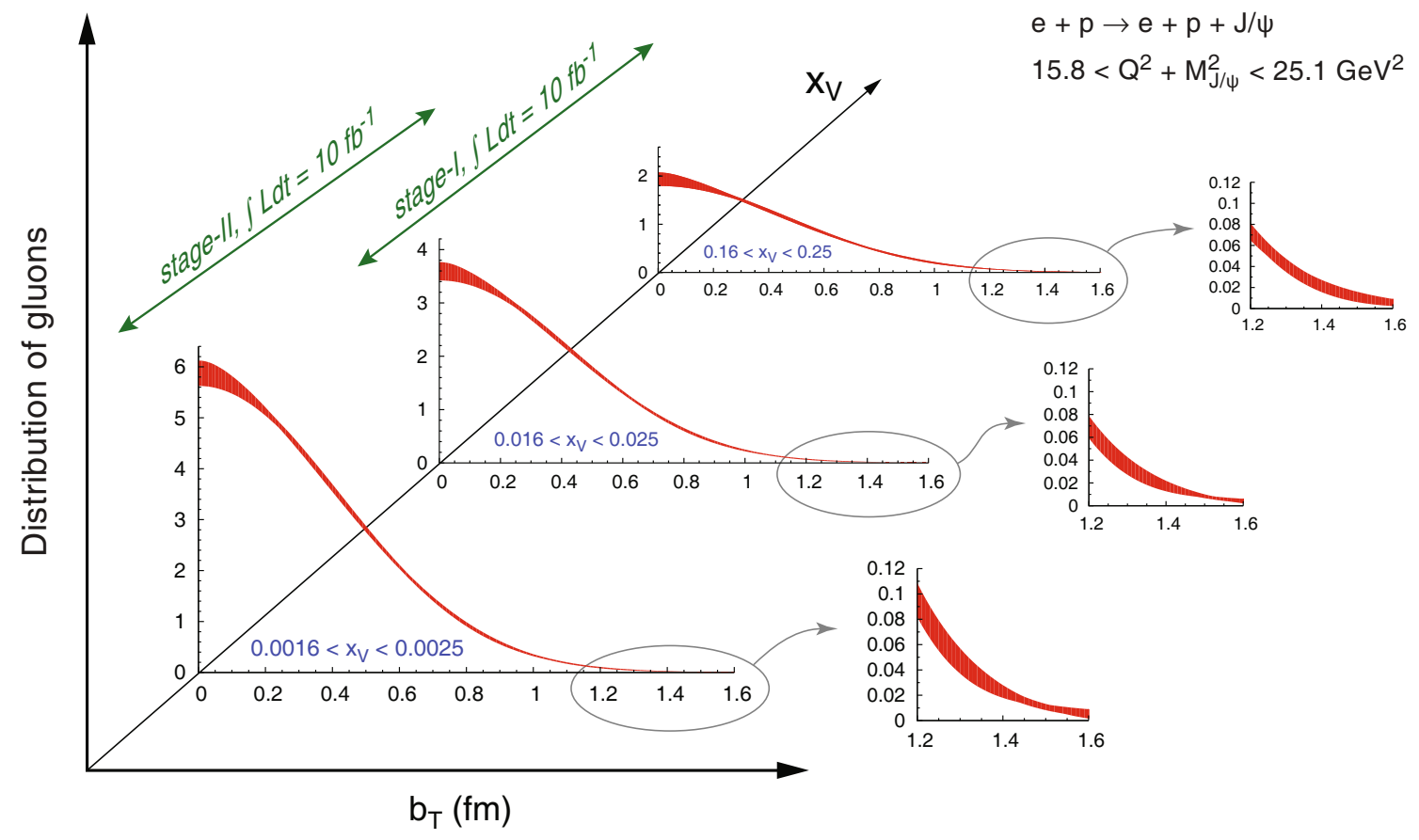

Fig. 2. The projected precision of the transverse spatial distribution of gluons as obtained from the cross-section of the exclusive $J / \psi$ production. It includes statistical and systematic uncertainties due to extrapolation into the unmeasured region of momentum transfer to the scattered proton. The distance of the gluon from the center of the proton is $b_{T}$ in femtometers, and the kinematic quantity $x_{V}=x_{B}\left(1+M_{J / \psi}^{2} / Q^{2}\right)$ determines the gluon's momentum fraction. The collision energies assumed for the top large $x_{V}$ plot and the lower $x_{V}$ plots are $E_{e}=5,20 \mathrm{GeV}$ and $E_{p}=100,250 \mathrm{GeV}$, respectively.

little is known to date. TMDs thus allow us to investigate the full three-dimensional dynamics of the proton, going well beyond the information about longitudional momentum contained in conventional parton distributions. With both electron and nucleon beams polarized at collider energies, the EIC will dramatically advance our knowledge of the motion of confined gluons and sea quarks in ways not achievable at any existing or proposed facility.

Figure 17 (left) in sect. 2.3 shows the transversemomentum distribution of up quarks inside a proton moving in the $z$-direction (out of the page) with its spin polarized in the $y$-direction. The color code indicates the probability of finding the up quarks. The anisotropy in transverse momentum is described by the Sivers distribution function, which is induced by the correlation between the proton's spin direction and the motion of its quarks and gluons. While the figure is based on a preliminary extraction of this distribution from current experimental data, nothing is known about the spin and momentum correlations of the gluons and sea quarks. The achievable statistical precision of the quark Sivers function from EIC kinematics is also shown in fig. 21 in sect. 2.3. Currently no data exist for extracting such a picture in the gluondominated region in the proton. The EIC will be crucial to initiate and realize such a program.

The tomography of the nucleon - spatial imaging of gluons and sea quarks

By choosing particular final states in electron+proton scattering, the EIC will probe the transverse spatial dis- tribution of sea quarks and gluons in the fast-moving proton as a function of the parton's longitudinal-momentum fraction, $x$. This spatial distribution yields a picture of the proton that is complementary to the one obtained from the transverse-momentum distribution of quarks and gluons, revealing aspects of proton structure that are intimately connected with the dynamics of QCD at large distances.

With its broad range of collision energies, its high luminosity and nearly hermetic detectors, the EIC could image the proton with unprecedented detail and precision from small to large transverse distances. The accessible parton momentum fractions $x$ extend from a region dominated by sea quarks and gluons to one where valence quarks become important, allowing a connection to the precise images expected from the $12 \mathrm{GeV}$ upgrade at JLab and COMPASS at CERN. This is illustrated in fig. 2, which shows the precision expected for the spatial distribution of gluons as measured in the exclusive process: electron + proton $\rightarrow$ electron + proton $+J / \psi$.

The tomographic images obtained from cross-sections and polarization asymmetries for exclusive processes are encoded in generalized parton distributions (GPDs) that unify the concepts of parton densities and of elastic form factors. They contain detailed information about spinorbit correlations and the angular momentum carried by partons, including their spin and their orbital motion. The combined kinematic coverage of the EIC and of the upgraded CEBAF as well as COMPASS is essential for extracting quark and gluon angular-momentum contributions to the proton's spin. 

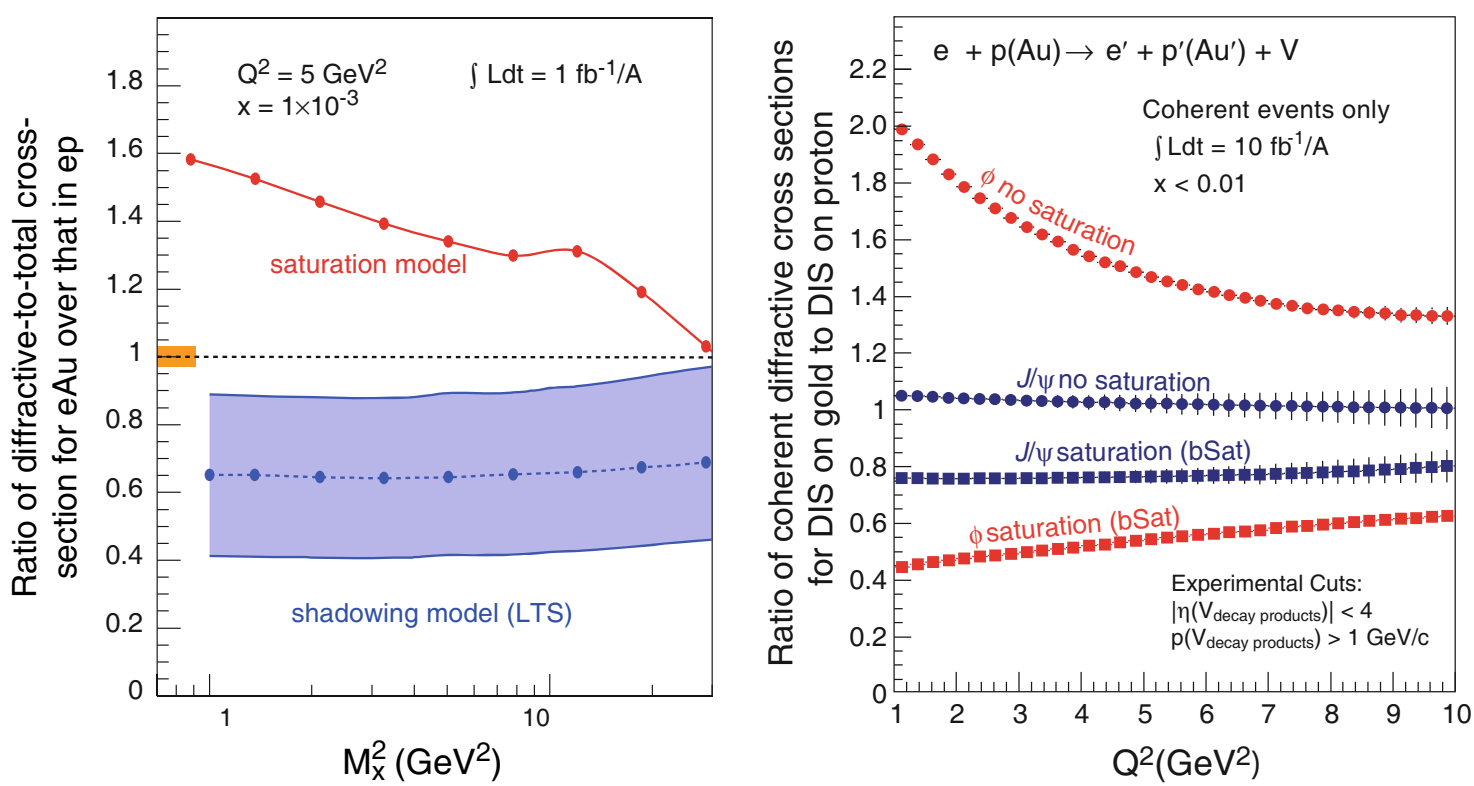

Fig. 3. Left: the ratio of diffractive over total cross-section for DIS on gold normalized to DIS on proton plotted for different values of $M_{X}^{2}$, the mass squared of hadrons produced in the collisions for models assuming saturation and non-saturation. The statistical error bars are too small to depict and the projected systematic uncertainty for the measurements is shown by the orange bar. The theoretical uncertainty for the predictions of the LTS model is shown by the grey band. Right: the ratio of the coherent diffractive cross-section in $e+\mathrm{Au}$ to $e+p$ collisions normalized by $A^{4 / 3}$ and plotted as a function of $Q^{2}$ for both saturation and non-saturation models. The $1 / Q$ is effectively the initial size of the quark-antiquark systems $(\phi$ and $J / \psi)$ produced in the medium.

\subsubsection{The nucleus, a QCD laboratory}

The nucleus is a QCD "molecule", with a complex structure corresponding to bound states of nucleons. Understanding the formation of nuclei in QCD is an ultimate long-term goal of nuclear physics. With its wide kinematic reach, as shown in fig. 32 (left) in sect. 3.1 , the capability to probe a variety of nuclei in both inclusive and semi-inclusive DIS measurements, the EIC will be the first experimental facility capable of exploring the internal 3-dimensional sea quark and gluon structure of a fastmoving nucleus. Furthermore, the nucleus itself is an unprecedented QCD laboratory for discovering the collective behavior of gluonic matter at an unprecedented occupation number of gluons, and for studying the propagation of fast-moving color charges in a nuclear medium.

\section{QCD at extreme parton densities}

In QCD, the large soft-gluon density enables the nonlinear process of gluon-gluon recombination to limit the density growth. Such a QCD self-regulation mechanism necessarily generates a dynamic scale from the interaction of high-density massless gluons, known as the saturation scale, $Q_{s}$, at which gluon splitting and recombination reach a balance. At this scale, the density of gluons is expected to saturate, producing new and universal properties of hadronic matter. The saturation scale $Q_{s}$ separates the condensed and saturated soft gluonic matter from the dilute, but confined, quarks and gluons in a hadron, as shown in fig. 39 in sect. 3.2 .
The existence of such a state of saturated, soft gluon matter, often referred to as the Color Glass Condensate (CGC), is a direct consequence of gluon self-interactions in $\mathrm{QCD}$. It has been conjectured that the $\mathrm{CGC}$ of $\mathrm{QCD}$ has universal properties common to nucleons and all nuclei, which could be systematically computed if the dynamic saturation scale $Q_{s}$ is sufficiently large. However, such a semi-hard $Q_{s}$ is difficult to reach unambiguously in electron-proton scattering without a multi- $\mathrm{TeV}$ proton beam. Heavy-ion beams at the EIC could provide precocious access to the saturation regime and the properties of the CGC because the virtual photon in forward lepton scattering probes matter coherently over a characteristic length proportional to $1 / x$, which can exceed the diameter of a Lorentz-contracted nucleus. Then, all gluons at the same impact parameter of the nucleus, enhanced by the nuclear diameter proportional to $A^{1 / 3}$ with the atomic weight $A$, contribute to the probed density, reaching saturation at far lower energies than would be needed in electron+proton collisions. While HERA, RHIC and the LHC have only found hints of saturated gluonic matter, the EIC would be in a position to seal the case, completing the process started at those facilities.

Figure 3 illustrates some of the dramatic predicted effects of gluon density saturation in electron+nucleus $v s$. electron+proton collisions at an EIC. The left frame considers coherent diffractive processes, defined to include all events in which the beam nucleus remains intact and there is a rapidity gap containing no produced particles. As shown in the figure, the fraction of such diffractive events are greatly enhanced by gluon saturation (the red 
points) in comparison with the predictions of shadowing model (the blue points). In all gluon saturation models, the coherent destructive multiple interaction among colored gluons suppresses both the coherent diffractive and total DIS cross-sections on nuclei compared to those on the proton, but, the suppression on the coherent diffractive events with the nucleus remained intact is much weaker than that of the total cross-section leading to a dramatic enhancement in the double ratio as shown in fig. 3 (left). An early measurement of coherent diffraction in $e+A$ collisions at the EIC would provide the first unambiguous evidence for gluon saturation.

Figure 3 (right) shows that gluon saturation is predicted to suppress vector meson production in $e+A$ relative to $e+p$ collisions at the EIC. The vector mesons result from quark-antiquark pair fluctuations of the virtual photon, which hadronize upon the exchange of gluons with the beam proton or nucleus. The magnitude of the suppression depends on the size (or color dipole moment) of the quark-antiquark pair, being significantly larger for produced $\phi$ (red points) than for $J / \psi$ (blue) mesons. An EIC measurement of the processes in fig. 3 (right) will provide a powerful probe to explore the properties of saturated gluon matter.

\section{The tomography of the nucleus}

With its capability to measure the diffractive and exclusive processes with a variety of ion beams, the EIC will also provide the first 3-dimensional images of sea quarks and gluons in a fast-moving nucleus with sub-femtometer resolution. For example, the EIC could obtain the spatial distribution of gluons in a nucleus by measuring the coherent diffractive production of $J / \psi$ in electron-nucleus scattering, similar to the case of electron-proton scattering shown in fig. 2 .

\section{Propagation of a color charge in QCD matter}

One of the key pieces of evidence for the discovery of the quark gluon plasma (QGP) at RHIC is jet quenching, manifested as a strong suppression of fast-moving hadrons produced in the very hot matter created in relativistic heavy-ion collisions. The suppression is believed to be due to the energy loss of colored partons traversing the QGP. It has been puzzling that the production is nearly as much suppressed for heavy as for light mesons, even though a heavy quark is much less likely to lose its energy via medium-induced radiation of gluons. Some of the remaining mysteries surrounding heavy vs. light quark interactions in hot matter can be illuminated by EIC studies of related phenomena in a better known cold nuclear matter. For example, the variety of ion beams available for electron-nucleus collisions at the EIC would provide a femtometer filter to test and to help determine the correct mechanism by which quarks and gluons lose energy and hadronize in nuclear matter (see schematic in fig. 57 (left)) in sect. 3.3 .

Figure 58 in sect. 3.3 shows the ratio of the number of produced mesons in electron+nucleus and electron+deuteron collisions for pions (light mesons) and $D^{0}$ - mesons (heavy mesons) at both low and high virtual photon energy $\nu$, as a function of $z$ - that is, the momentum fraction of the virtual photon taken by the observed meson. The calculation of the lines and blue circle symbols assumes that the mesons are formed outside of the nucleus, as shown in the top sketch of fig. 57 (left), while the square symbols are simulated according to a model where a color neutral pre-hadron was formed inside the nucleus, like in the bottom sketch of fig. 57 (left). The location of measurements within the shaded area would provide the first direct information on when the mesons are formed. Unlike the suppression expected for pion production at all $z$, the ratio of heavy-meson production could be larger than unity due to very different hadronization properties of heavy mesons. The discovery of such a dramatic difference in multiplicity ratios between light and heavy mesons at the EIC will shed light on the hadronization process and on what governs the transition from quarks to hadrons.

\section{The distribution of quarks and gluons in the nucleus}

The EMC experiment at CERN and experiments in the following two decades clearly revealed that the distribution of quarks in a fast-moving nucleus is not a simple superposition of their distributions within nucleons. Instead, the ratio of nuclear over nucleon structure functions follows a non-trivial function of Bjorken $x$, deviating significantly from unity, with a suppression as $x$ decreases (often referred to as nuclear shadowing). Amazingly, there is as of yet no knowledge whether the same holds true for gluons. With its much wider kinematic reach in both $x$ and $Q$, the EIC could measure the suppression of the structure functions to a much lower value of $x$, approaching the region of gluon saturation. In addition, the EIC could for the first time reliably quantify the nuclear gluon distribution over a wide range of momentum fraction $x$.

\subsubsection{Physics possibilities at the intensity frontier}

The subfield of Fundamental Symmetries in nuclear physics has an established history of key discoveries, enabled by either the introduction of new technologies or the increase in energy and luminosity of accelerator facilities. While the EIC is primarily being proposed for exploring new frontiers in QCD, it offers a unique new combination of experimental probes potentially interesting to the investigations in Fundamental Symmetries. For example, the availability of polarized beams at high energy and high luminosity, combined with a state-of-the-art hermetic detector, could extend Standard Model tests of the running of the weak-coupling constant far beyond the reach of the JLab12 parity violation program, namely toward the $Z$ pole scale previously probed at LEP and SLC.

\subsection{The EIC and its realization}

Two independent designs for a future EIC have evolved in the United States. Both use the existing infrastructure 


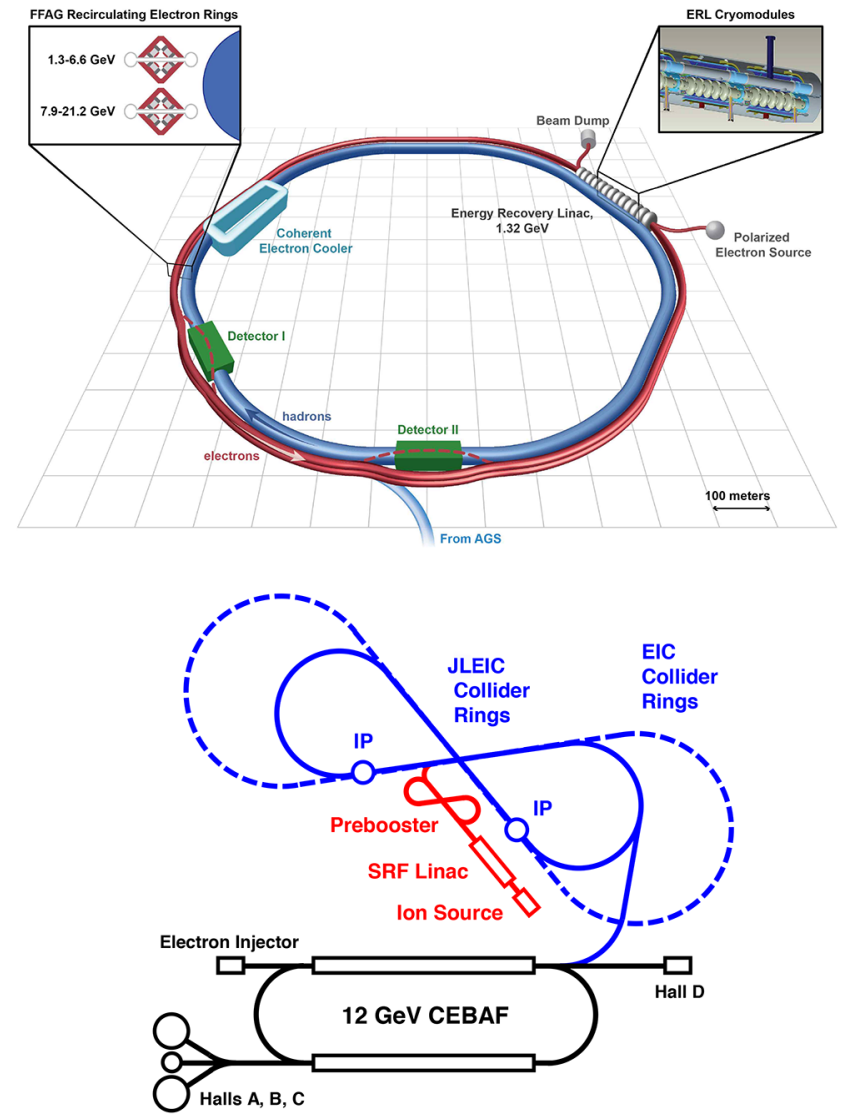

Fig. 4. Top: the schematic of eRHIC at BNL, which would require construction of an electron beam facility (red) to collide with the RHIC blue beam at up to three interaction points. Bottom: the schematic of JLEIC at JLab, which would require construction of an ion linac (red), and an electron-ion collider ring (blue) with at least two interaction points, around the $12 \mathrm{GeV}$ CEBAF.

and facilities available to the US nuclear science community. At Brookhaven National Laboratory (BNL), the eRHIC design (fig. 4, top) utilizes a new electron beam facility based on an Energy Recovery LINAC (ERL) to be built inside the RHIC tunnel to collide with RHICs existing high-energy polarized proton and nuclear beams. At Jefferson Laboratory (JLab), its Electron Ion Collider (JLEIC) design (fig. 4, bottom) employs a new electron and ion collider ring complex together with the $12 \mathrm{GeV}$ upgraded CEBAF, now under construction, to achieve similar collision parameters.

The EIC machine designs are aimed at achieving

- highly polarized ( 70\%) electron and nucleon beams;

- ion beams from deuteron to the heaviest nuclei (uranium or lead);

- variable center-of-mass energies from $\sim 20-\sim 100 \mathrm{GeV}$, upgradable to $\sim 140 \mathrm{GeV}$;

- high collision luminosity $\sim 10^{33-34} \mathrm{~cm}^{-2} \mathrm{~s}^{-1}$;

- possibilities of having more than one interaction region.
The EIC requirements will push accelerator designs to the limits of the current technology, and will therefore need significant R\&D. Cooling of the hadron beam is essential to attain the luminosities demanded by the science. The development of coherent electron cooling is now underway at BNL, while the JLab design is based on conventional electron cooling techniques, but proposes to extend them to significantly higher energy and to use bunched electron beams for the first time.

An energy recovery linac at the highest possible energy and intensity is key to the realization of eRHIC at BNL, and this technology is also important for electron cooling in JLEIC at JLab. The eRHIC design at BNL also requires a high-intensity polarized electron source that would be an order of magnitude higher in intensity than the current state of the art, while the JLEIC design at JLab will utilize a novel figure- 8 storage ring design for both electrons and ions.

The physics-driven requirements on the EIC accelerator parameters and extreme demands on the kinematic coverage for measurements makes integration of the detector into the accelerator a particularly challenging feature of the design. Lessons learned from past experience at HERA have been considered while designing the EIC interaction region. Driven by the demand for high precision on particle detection and identification of final-state particles in both $e+p$ and $e+\mathrm{A}$ programs, modern particle detector systems will be at the heart of the EIC. In order to keep the detector costs manageable, R\&D efforts are under way on various novel ideas for: compact (fiber sampling and crystal) calorimetry, tracking (NaI coated GEMs, GEM size and geometries), particle identification (compact DIRC, dual radiator RICH and novel TPC) and high radiation tolerance for electronics. Meeting these R\&D challenges will keep the US nuclear science community at the cutting edge in both accelerator and detector technology.

\subsection{Physics deliverables of the EIC}

Both realizations of the EIC, the eRHIC and the JLEIC, are expected to evolve over time from $\sim 20-100 \mathrm{GeV}$ in center-of-mass energy to $\sim 140 \mathrm{GeV}$ with polarized nucleon and electron beams, a wide range of heavy-ion beams for nuclear DIS, and a luminosity for electron+proton collisions approaching $10^{34} \mathrm{~cm}^{-2} \mathrm{~s}^{-1}$. With such a facility, the EIC physics program would have an excellent start toward addressing the following fundamental questions with key measurements:

- Proton spin: Within just a few months of operation, the EIC would be able to deliver decisive measurements, which no other facility in the world could achieve, on how much the intrinsic spin of quarks and gluons contribute to the proton spin as shown in fig. 13.

- The motion of quarks and gluons in the proton: Semiinclusive measurements with polarized beams would enable us to selectively probe with precision the correlation between the spin of a fast-moving proton and 
the confined transverse motion of both quarks and gluons within. Images and profile in momentum space as shown in figs. 17 and 21 are simply unattainable without the polarized electron and proton beams of the proposed EIC.

- The tomographic images of the proton: By measuring exclusive processes, the EIC, with its unprecedented luminosity and detector coverage, would create detailed images of the proton gluonic matter distribution, as shown in fig. 2, as well as images of sea quarks. Such measurements would reveal aspects of proton structure that are intimately connected with QCD dynamics at large distances.

- QCD matter at an extreme gluon density: By measuring the diffractive cross-sections together with the total DIS cross-sections in electron+proton and electron+ nucleus collisions as shown in fig. 3, the EIC would provide the first unambiguous evidence for the novel QCD matter of saturated gluons. The EIC is poised to explore with precision the new field of the collective dynamics of saturated gluons at high energies.

- Quark hadronization: By measuring pion and $D^{0}$ meson production in both electron+proton and electron+nucleus collisions, the EIC would provide the first measurement of the quark mass dependence of the hadronization along with the response of nuclear matter to a fast-moving quark.

The Relativistic Heavy-Ion Collider (RHIC) at BNL has revolutionized our understanding of hot and dense QCD matter through its discovery of the strongly-coupled quark gluon plasma that existed a few microseconds after the birth of the universe. Unprecedented studies of the nucleon and nuclear structure -including the nucleon spin, and the nucleon's tomographic images in the valence quark region - have been and will be possible with the high luminosity fixed target experiments at Jefferson Laboratory using the 6 and $12 \mathrm{GeV}$ CEBAF, respectively. The EIC promises to propel both programs to the next QCD frontier, by unraveling the three-dimensional sea quark and gluon structure of visible matter. Furthermore, the EIC will probe the existence of a universal state of saturated gluon matter and has the capability to explore it in detail. The EIC will thus enable the US to continue its leadership role in nuclear science research through the quest for understanding the unique gluon-dominated nature of visible matter in the universe.

\section{Spin and three-dimensional structure of the nucleon}

\subsection{Introduction}

Among the most intriguing aspects of quantum chromodynamics (QCD) is the relation between its basic degrees of freedom, quarks and gluons, and the observable physical states, i.e. hadrons such as the proton. Parton distributions are the most prominent quantities that describe this relationship. They are relevant in connection with several key issues of the strong interaction:

- What is the dynamical origin of sea quarks and gluons inside the proton?

Parton distributions describe the proton as a system of many quarks, anti-quarks and gluons. At high resolution, the presence of partons with small momentum fraction $x$ can largely be understood as the result of parton radiation, similar to the appearance of electrons, positrons and photons generated from a single electron in an electromagnetic cascade. This parton radiation can be computed using perturbation theory in the small coupling $\left(\alpha_{s}\right)$ limit. However, comparison with experimental data shows that even at low resolution, the proton does not only consist of quarks carrying about a third of the proton momentum, as one might naively expect from the familiar constituent quark picture, where the proton is made up of two $u$ quarks and one $d$ quark. Instead, even at low resolution, the proton contains both gluons and low-momentum quarks and anti-quarks (termed sea quarks) $[3,4]$. These must be generated by dynamics beyond the reach of perturbation theory, and their origin remains to be understood. Note that calculations in lattice QCD tell us that even the proton mass is largely due to the binding energy of the gluons that keep the quarks together.

- How does the proton spin originate at the microscopic level?

The fact that quarks have spin $1 / 2$ and gluons spin 1 plays an essential role in their interactions among themselves. An outstanding question is how the total spin of the proton is built up from the polarization and the orbital angular momentum of quarks, anti-quarks and gluons. Starting with the seminal results of the EMC experiment [5], a series of increasingly precise measurements in the last decades revealed that the polarization of the quarks and anti-quarks combined, only provides about $30 \%$ of the nucleon spin. Present lattice calculations [6] suggest that the missing $70 \%$ is not provided by the orbital angular momentum of quarks alone, and recent results from RHIC point towards a significant contribution from the polarization of gluons [7]. This highlights again the importance of gluons for the basic properties of the nucleon.

- How is hadron structure influenced by chiral symmetry and its breaking?

QCD has an approximate chiral symmetry, which is dynamically broken. As a consequence the strong interaction generates Goldstone bosons - the pionswhose mass is remarkably small compared with that of other hadrons. These almost massless bound states propagate over distances significantly larger than the typical hadronic scale. They are critical in generating the force that binds neutrons and protons within nuclei, but also appear to greatly influence the properties of isolated nucleons. No understanding of matter is complete without a detailed explanation of the role 
of pions. It is thus crucial to expose the role played by pions in nucleon structure.

- How does confinement manifest itself in the structure of hadrons?

At distances around 1 femtometer (fm) the strong force becomes so strong that quarks and gluons are confined in hadrons and cannot exist as free particles. As a consequence, the structure of the proton differs profoundly from that of weakly bound systems such as atoms (whose overall size is proportional to the inverse electron mass). The spatial distribution of partons in the proton and their distribution in transverse momentum is characterized by scales of the order of a fm or a few hundred $\mathrm{MeV}$, which are similar to the confinement scale and very different from the $u$ and $d$ quark masses. Experimental mapping and theoretical computation of these distributions should further our understanding of confinement.

The EIC will be unique in mapping out the quark-gluon structure of the proton in several ways that will take our knowledge to a new level. Specifically, the EIC will enable us to investigate:

- the distribution of sea quarks and gluons in momentum and in position space, in order to better understand their dynamical interplay;

- their polarization and their orbital angular momentum, the latter being closely connected with their transverse position and transverse motion since it is a cross product $(\boldsymbol{L}=\boldsymbol{r} \times \boldsymbol{p})$;

- correlations between polarization and the distribution of partons in momentum or position space, which may be regarded as the QCD analog of spin-orbit correlations in atomic or nuclear physics;

- the change of distributions when going from small to large $x$, to compare the characteristics of sea and valence quarks and to understand their relation to each other;

- the dependence of the above characteristics on the quark flavor. This is of particular interest when comparing distributions, i.e. $\bar{u}$ with $\bar{d}, \bar{s}$ with $(\bar{u}+\bar{d}) / 2$ or $s$ with $\bar{s}$. Significant differences between those distributions are a direct imprint of non-perturbative dynamics because perturbative parton radiation is not able to generate them. This imparts special interest to the polarization carried by sea quarks of different flavors, above and beyond its contribution to the overall spin of the proton.

To quantify these properties and to connect them with experimental data, we have a powerful formalism at our disposal, which has seen significant progress in the last one and a half decades. Parton distributions come in different varieties, with an increasing level of complexity:

- The familiar parton distribution functions (PDFs) $f(x)$ give the number density of partons with longitudinal momentum fraction $x$ in a fast-moving proton, where the longitudinal direction is given by the proton momentum. They are measured in inclusive

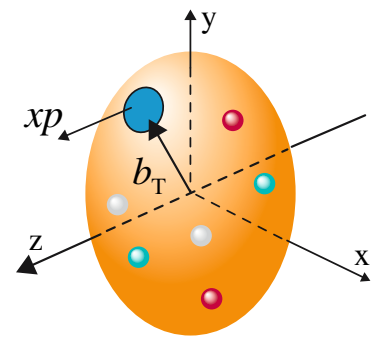

Fig. 5. Schematic view of a parton with longitudinal momentum fraction $x$ and transverse position $\boldsymbol{b}_{T}$ in the proton.

or semi-inclusive processes, the first and foremost being inclusive deep inelastic lepton-proton scattering (DIS). PDFs form the backbone of our knowledge about hadron structure, and for most cases their determination is an enterprise at the precision frontier. A powerful tool for disentangling the distributions for different quark and anti-quark flavors is semi-inclusive DIS (SIDIS), where a specified hadron is detected in the final state. SIDIS involves fragmentation functions, which describe another key phenomenon of the strong interaction, namely the fragmentation of a parton into a hadron. Fragmentation functions and parton distributions provide two different settings to investigate the consequences of confinement. The possibilities to study the fragmentation process in nuclei will be discussed in sect. 3.3 .

- Transverse-momentum-dependent parton densities (TMDs) $f\left(x, \boldsymbol{k}_{T}\right)$ describe the joint distribution of partons in their longitudinal-momentum fraction $x$ and their momentum $\boldsymbol{k}_{T}$ transverse to the proton direction. To measure TMDs requires more detailed information about the kinematics of a scattering process. In the appropriate kinematics of SIDIS, the transverse momentum of the detected final-state hadron can be computed from a $k_{T}$-dependent parton density and from a $k_{T}$-dependent fragmentation function, which describes the transverse momentum transferred during the hadronization process.

- Generalized parton distributions (GPDs) $H(x, \xi, t)$ appear in exclusive scattering processes such as deeply virtual Compton scattering (DVCS: $\gamma^{*} p \rightarrow \gamma p$ ), in which all final-state particles are detected. They depend on two longitudinal momentum fractions $x$ and $\xi$ (see Sidebar IV) and on the squared momentum transfer $t$ to the proton or equivalently, on its transverse component $\boldsymbol{\Delta}_{T}$. Setting $\xi=0$ and performing a Fourier transform with respect to $\boldsymbol{\Delta}_{T}$ one obtains an impact parameter distribution $f\left(x, \boldsymbol{b}_{T}\right)$, which describes the joint distribution of partons in their longitudinal momentum and their transverse position $\boldsymbol{b}_{T}$ inside the proton, as sketched in fig. 5 .

Integrating the generalized quark distribution $H(x$, $0, t)$ over $x$ and taking an appropriate sum over quark flavors, one obtains the electromagnetic Dirac form factor $F_{1}(t)$ of the proton. This provides a connection between parton distributions and form factors, which have played a major role in exploring the proton struc- 


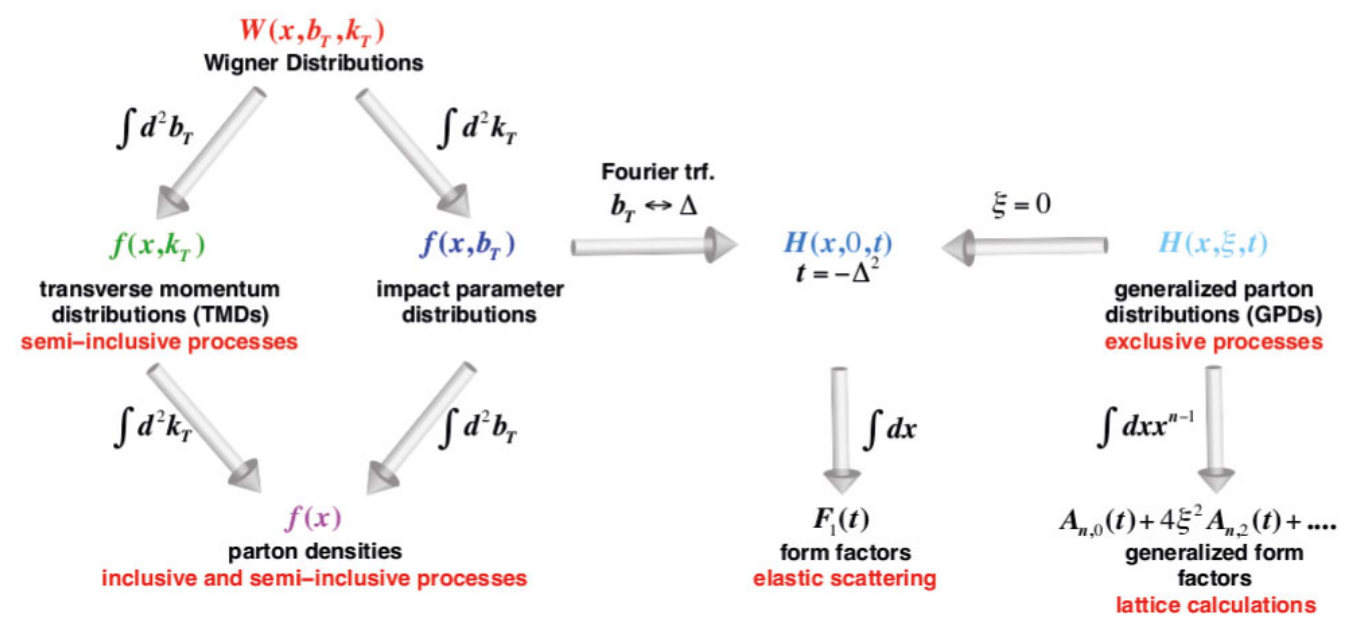

Fig. 6. Connections between different quantities describing the distribution of partons inside the proton. The functions given here are for unpolarized partons in an unpolarized proton; analogous relations hold for polarized quantities.

ture ever since the seminal experiment of Hofstadter. More generally, the integral $\int \mathrm{d} x x^{n-1} H(x, \xi, t)$ gives generalized form factors for a large set of local operators that cannot be directly measured but can be computed on the lattice. This provides a connection with one of the main tools for calculations in the nonperturbative sector of QCD.

Indeed, measurements at the EIC and lattice calculations will have a high degree of complementarity. For some quantities, notably the $x$ moments of unpolarized and polarized quark distributions, a precise determination will be possible both in experiment and on the lattice. Using this to validate the methods used in lattice calculations, one will gain confidence in computing quantities whose experimental determination is very hard, such as generalized form factors. Furthermore, one can gain insight into the underlying dynamics by computing the same quantities with values of the quark masses that are not realized in nature, so as to reveal the importance of these masses for specific properties of the nucleon. On the other hand, there are many aspects of hadron structure beyond the reach of lattice computations, in particular, the distribution and polarization of quarks and gluons at small $x$, for which collider measurements are our only source of information.

Both impact parameter distributions $f\left(x, \boldsymbol{b}_{T}\right)$ and transverse-momentum distributions $f\left(x, \boldsymbol{k}_{T}\right)$ describe proton structure in three dimensions, or more accurately in $2+1$ dimensions (two transverse dimensions in either configuration or momentum space, along with one longitudinal dimension in momentum space). Note that in a fastmoving proton, the transverse variables play very different roles than the longitudinal momentum.

It is important to realize that $f\left(x, \boldsymbol{b}_{T}\right)$ and $f\left(x, \boldsymbol{k}_{T}\right)$ are not related to each other by a Fourier transform (nevertheless it is common to denote both functions by the same symbol $f)$. Instead, $f\left(x, \boldsymbol{b}_{T}\right)$ and $f\left(x, \boldsymbol{k}_{T}\right)$ give complementary information about partons, and both types of quantities can be thought of as descendants of Wigner distributions $W\left(x, \boldsymbol{b}_{T}, \boldsymbol{k}_{T}\right)$ [8], which are used extensively in other branches of physics [9]. Although there is no known way to measure Wigner distributions for quarks and gluons, they provide a unifying theoretical framework for the different aspects of hadron structure we have discussed. Figure 6 shows the connection between these different aspects and the experimental possibilities to explore them.

All parton distributions depend on a scale which specifies the resolution at which partons are resolved, and which in a given scattering process is provided by a large momentum transfer. For many processes in $e+p$ collisions, the relevant hard scale is $Q^{2}$ (see Sidebar I). The evolution equations that describe the scale dependence of parton distributions provide an essential tool, both for the validation of the theory and for the extraction of parton distributions from cross section data. They also allow one to convert the distributions seen at high resolution to lower resolution scales, where contact can be made with non-perturbative descriptions of the proton.

An essential property of any particle is its spin, and parton distributions can depend on the polarization of both the parton and the parent proton. The spin structure is particularly rich for TMDs and GPDs because they single out a direction in the transverse plane, thus opening the way for studying correlations between spin and $\boldsymbol{k}_{T}$ or $\boldsymbol{b}_{T}$. Information about transverse degrees of freedom is essential to access orbital angular momentum, and specific TMDs and GPDs quantify the orbital angular momentum carried by partons in different ways.

The theoretical framework we have sketched is valid over a wide range of momentum fractions $x$, connecting in particular the region of valence quarks with the one of gluons and the quark sea. While the present section is focused on the nucleon, the concept of parton distributions is well adapted to study the dynamics of partons in nuclei, as we will see in sect. 3.3. For the regime of small $x$, which is probed in collisions at the highest energies, a different theoretical description is at our disposal. Rather than parton distributions, a basic quantity in this approach is the amplitude for the scattering of a color dipole on a proton or a nucleus. The joint distribution of gluons in $x$ and 


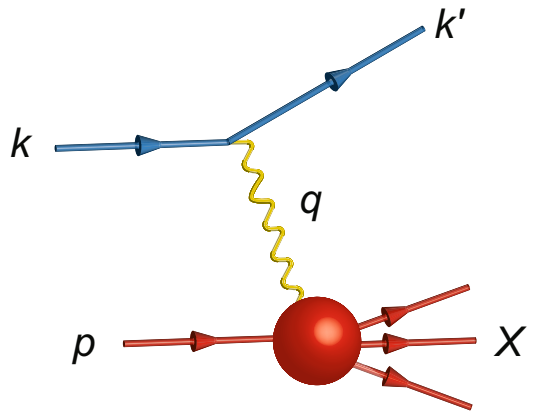

Fig. 7. A schematic diagram of the Deep Inelastic Scattering (DIS) process.

in $\boldsymbol{k}_{T}$ or $\boldsymbol{b}_{T}$ can be derived from this dipole amplitude. This high-energy approach is essential for addressing the physics of high parton densities and of parton saturation, as discussed in sect. 3.2. On the other hand, in a regime of moderate $x$, around $10^{-3}$ for the proton and higher for heavy nuclei, the theoretical descriptions based on either parton distributions or color dipoles are both applicable and can be related to each other. This will provide us with valuable flexibility for interpreting data in a wide kinematic regime.

The following sections highlight the physics opportunities in measuring PDFs, TMDs and GPDs to map out the quark-gluon structure of the proton at the EIC. An essential feature throughout will be the broad reach of the $\mathrm{EIC}$ in the kinematic plane of the Bjorken variable $x$ (see Sidebar I) and the invariant momentum transfer $Q^{2}$ to the electron. While $x$ determines the momentum fraction of the partons probed, $Q^{2}$ specifies the scale at which the partons are resolved. Wide coverage in $x$ is hence essential for going from the valence quark regime deep into the region of gluons and sea quarks, whereas a large lever arm in $Q^{2}$ is the key for unraveling the information contained in the scale evolution of parton distributions.

\section{Sidebar I. Deep Inelastic Scattering: kinematics}

\section{Deep Inelastic Scattering}

$e(k)+p \longrightarrow e\left(k^{\prime}\right)+X$, as sketched in fig. 7 , proceeds through the exchange of a virtual photon between the electron and the proton. The kinematic description remains the same for the exchange of a $Z$ or $W$ boson, which becomes important at high momentum transfer.

Depending on the physics situation, the process is discussed in different reference frames:

- the collider frame, where a proton with energy $E_{p}$ and an electron with energy $E_{e}$ collide head-on;

- the rest frame of the hadronic system $X$, i.e. the center-of-mass of the $\gamma^{*} p$ collision;

- the rest frame of the proton.

\section{Kinematic variables}

In the following, we neglect the proton mass, $M$, where appropriate and the electron mass throughout.

$k, k^{\prime}$ are the four-momenta of the incoming and outgoing lepton;

$p$ is the four-momentum of a nucleon.

Lorentz invariants

- the squared $e+p$ collision energy $s=(p+k)^{2}=4 E_{p} E_{e}$

- the squared momentum transfer to the lepton $Q^{2}=$ $-q^{2}=-\left(k-k^{\prime}\right)^{2}$, equal to the virtuality of the exchanged photon. Large values of $Q^{2}$ provide a hard scale to the process, which allows one to resolve quarks and gluons in the proton.

- the Bjorken variable $x_{B}=Q^{2} /(2 p \cdot q)$, often simply denoted by $x$. It determines the momentum fraction of the parton on which the photon scatters. Note that $0<x<1$ for $e+p$ collisions.

- the inelasticity $y=(q \cdot p) /(k \cdot p)$ is limited to values $0<$ $y<1$ and determines in particular the polarization of the virtual photon. In the collider frame, the energy of the scattered electron is $E_{e}^{\prime}=E_{e}(1-y)+Q^{2} /\left(4 E_{e}\right)$; detection of the scattered electron thus typically requires a cut on $y<y_{\max }$.

These invariants are related by $Q^{2}=x y s$. The available phase space is often represented in the plane of $x$ and $Q^{2}$. For a given $e+p$ collision energy, lines of constant $y$ are then lines with a slope of 45 degrees in a double logarithmic $x-Q^{2}$-plot.

\section{Two more important variables}

$W^{2}=(p+q)^{2}=Q^{2}(1-1 / x)$ is the squared invariant mass of the produced hadronic system $X$.

DIS is characterized by the Bjorken limit, where $Q^{2}$ and $W^{2}$ become large at a fixed value of $x$. Note: for a given $Q^{2}$, small $x$ corresponds to a high $\gamma^{*} p$ collision energy.

$\nu=q \cdot p / M=y s /(2 M)$ is the energy lost by the lepton (i.e. the energy carried away by the virtual photon) in the proton rest frame.

For scattering on a nucleus of atomic number $A$, replace the proton momentum $p$ by $P / A$ in the definitions, where $P$ is the momentum of the nucleus. Note that for the Bjorken variable one then has $0<x<A$.

\section{Sidebar II. Deep Inelastic Scattering: structure functions}

The cross-sections for neutral-current deep inelastic scattering $\left(e+N \longrightarrow e^{\prime}+X\right)$ on unpolarized nucleons and nuclei can be written in the one-photon exchange approximation (neglecting electroweak effects) in terms of two structure functions $F_{2}$ and $F_{L}$ :

$\frac{\mathrm{d}^{2} \sigma}{\mathrm{d} x \mathrm{~d} Q^{2}}=\frac{4 \pi \alpha^{2}}{x Q^{4}}\left[\left(1-y+\frac{y^{2}}{2}\right) F_{2}\left(x, Q^{2}\right)-\frac{y^{2}}{2} F_{L}\left(x, Q^{2}\right)\right]$. 
For practical purposes, often the reduced cross-section, $\sigma_{r}$, is used:

$$
\begin{aligned}
\sigma_{r} & =\left(\frac{\mathrm{d}^{2} \sigma}{\mathrm{d} x \mathrm{~d} Q^{2}}\right) \frac{x Q^{4}}{2 \pi \alpha^{2}\left[1+(1-y)^{2}\right]} \\
& =F_{2}\left(x, Q^{2}\right)-\frac{y^{2}}{1+(1-y)^{2}} F_{L}\left(x, Q^{2}\right) .
\end{aligned}
$$

For longitudinally polarized proton and electron beams, the neutral current cross-section for deep inelastic scattering can be written in terms of one structure function $g_{1}$ :

$$
\frac{1}{2}\left[\frac{\mathrm{d}^{2} \sigma^{\rightleftarrows}}{\mathrm{d} x \mathrm{~d} Q^{2}}-\frac{\mathrm{d}^{2} \sigma^{\rightrightarrows}}{\mathrm{d} x \mathrm{~d} Q^{2}}\right] \simeq \frac{4 \pi \alpha^{2}}{Q^{4}} y(2-y) g_{1}\left(x, Q^{2}\right),
$$

where the superscript arrows represent electron and proton longitudinal spin directions and the terms suppressed by $x^{2} M^{2} / Q^{2}$ have been neglected.

Experimentally $F_{2}, F_{L}$ and $g_{1}$ can be measured in inclusive scattering, i.e., the final hadronic state, $X$, does not need to be analyzed. The relevant kinematic variables $x, Q^{2}$, and $y$, can be reconstructed from the measured scattered lepton alone.

$F_{2}, F_{L}$ and $g_{1}$ are proportional to the cross-section for the hadronic subprocess $\gamma^{*}+p \rightarrow X$, which gets contributions from the different polarization states of the virtual photon. $F_{2}$ corresponds to the sum over transverse and longitudinal polarizations and the structure function $F_{L}$ to longitudinal polarization of the virtual photon (i.e., helicity $=0)$. The $g_{1}$ structure function is sensitive to the transverse polarization of the virtual photon (i.e., helicity $= \pm 1)$.

Equation (2) shows that the longitudinal structure function $F_{L}$ starts to contribute to the cross-section at larger values of $y$ but is negligible at very small values of $y$. To separate the structure functions $F_{L}$ and $F_{2}$ for a given $x$ and $Q^{2}$, one needs to measure the cross-section for different values of $y$ and hence different $e+p$ collision energies.

At large $Q^{2}$ and to leading order (LO) in the strong coupling $\alpha_{s}$, the structure functions $F_{2}$ and $g_{1}$ are respectively sensitive to the sum over unpolarized and longitudinally polarized quark and anti-quark distributions in the nucleon,

$$
\begin{aligned}
& F_{2}\left(x, Q^{2}\right)=x \sum e_{q}^{2}\left[q\left(x, Q^{2}\right)+\bar{q}\left(x, Q^{2}\right)\right], \\
& g_{1}\left(x, Q^{2}\right)=\frac{1}{2} \sum e_{q}^{2}\left[\Delta q\left(x, Q^{2}\right)+\Delta \bar{q}\left(x, Q^{2}\right)\right],
\end{aligned}
$$

where $e_{q}$ denotes a quark's electric charge.

At large $Q^{2}$, one has $F_{L}=0$ at LO, i.e., this structure function receives its first contributions at order $\alpha_{s}$. It is thus particularly sensitive to gluons, especially at low $x$ where the gluon densitiy is much larger than the densities for quarks and anti-quarks.

Figure 8 (left) shows the world data of the reduced cross-section, $\sigma_{r} \propto F_{2}$, as a function of $Q^{2}$ for a wide range of fixed values of $x$ for scattering on a proton [10].
The apparent scaling of the data with $Q^{2}$ at large $x$ in early DIS data from SLAC was termed "Bjorken scaling" and motivated the parton model. Violations of this scaling are predicted by the QCD evolution equations for parton densities. They are especially strong at small $x$. We note that our experimental knowledge of $F_{L}$ is considerably less precise than that of $F_{2}$.

Figure 8 (right) shows the world data of the polarised structure function $g_{1}$ as a function of $Q^{2}$ for fixed values of $x$ for scattering on a proton [11]. The covered $x-Q^{2}$ range is significantly smaller than that for the unpolarized measurements, which is due to the fact that there has been no collider with both polarized lepton and hadron beams.

\subsection{The longitudinal spin of the nucleon ${ }^{1}$}

\subsubsection{Introduction}

Deep inelastic processes, when carried out with longitudinally polarized nucleons, probe the helicity parton distribution functions of the nucleon. For each flavor $f=$ $u, d, s, \bar{u}, \bar{d}, \bar{s}, g$ these are defined by

$$
\Delta f\left(x, Q^{2}\right) \equiv f^{+}\left(x, Q^{2}\right)-f^{-}\left(x, Q^{2}\right),
$$

with $f^{+}\left(f^{-}\right)$denoting the number density of partons with the same (opposite) helicity as the nucleons, as a function of the momentum fraction $x$ and the resolution scale $Q$. Similar to the unpolarized quark and gluon densities, the $Q^{2}$-dependences of $\Delta q\left(x, Q^{2}\right), \Delta \bar{q}\left(x, Q^{2}\right)$ and the gluon helicity distribution $\Delta g\left(x, Q^{2}\right)$ are related by QCD radiative processes that are calculable [12-18].

When integrated over all momentum fractions and appropriately summed over flavors, the $\Delta f$ distributions give the quark and gluon spin contributions $S_{q}, S_{g}$ to the proton spin which appear in the fundamental proton helicity sum rule [19-22] (see [23] for a brief review and additional references):

$$
\frac{1}{2}=S_{q}+L_{q}+S_{g}+L_{g}
$$

Here, we have

$$
\begin{aligned}
S_{q}\left(Q^{2}\right)= & \frac{1}{2} \int_{0}^{1} \Delta \Sigma\left(x, Q^{2}\right) \mathrm{d} x \\
\equiv & \frac{1}{2} \int_{0}^{1}(\Delta u+\Delta \bar{u}+\Delta d+\Delta \bar{d} \\
& +\Delta s+\Delta \bar{s})\left(x, Q^{2}\right) \mathrm{d} x \\
S_{g}\left(Q^{2}\right)= & \int_{0}^{1} \Delta g\left(x, Q^{2}\right) \mathrm{d} x,
\end{aligned}
$$

where the factor $1 / 2$ in the first equation is the spin of each quark and anti-quark. The $\Delta f$ distributions are thus key ingredients to solving the proton spin problem.

As discussed in Sidebar II, experimental access to the $\Delta f$ in lepton-scattering is obtained through the spindependent structure function $g_{1}\left(x, Q^{2}\right)$, which appears in

${ }^{1}$ Conveners: Ernst Sichtermann and Werner Vogelsang. 

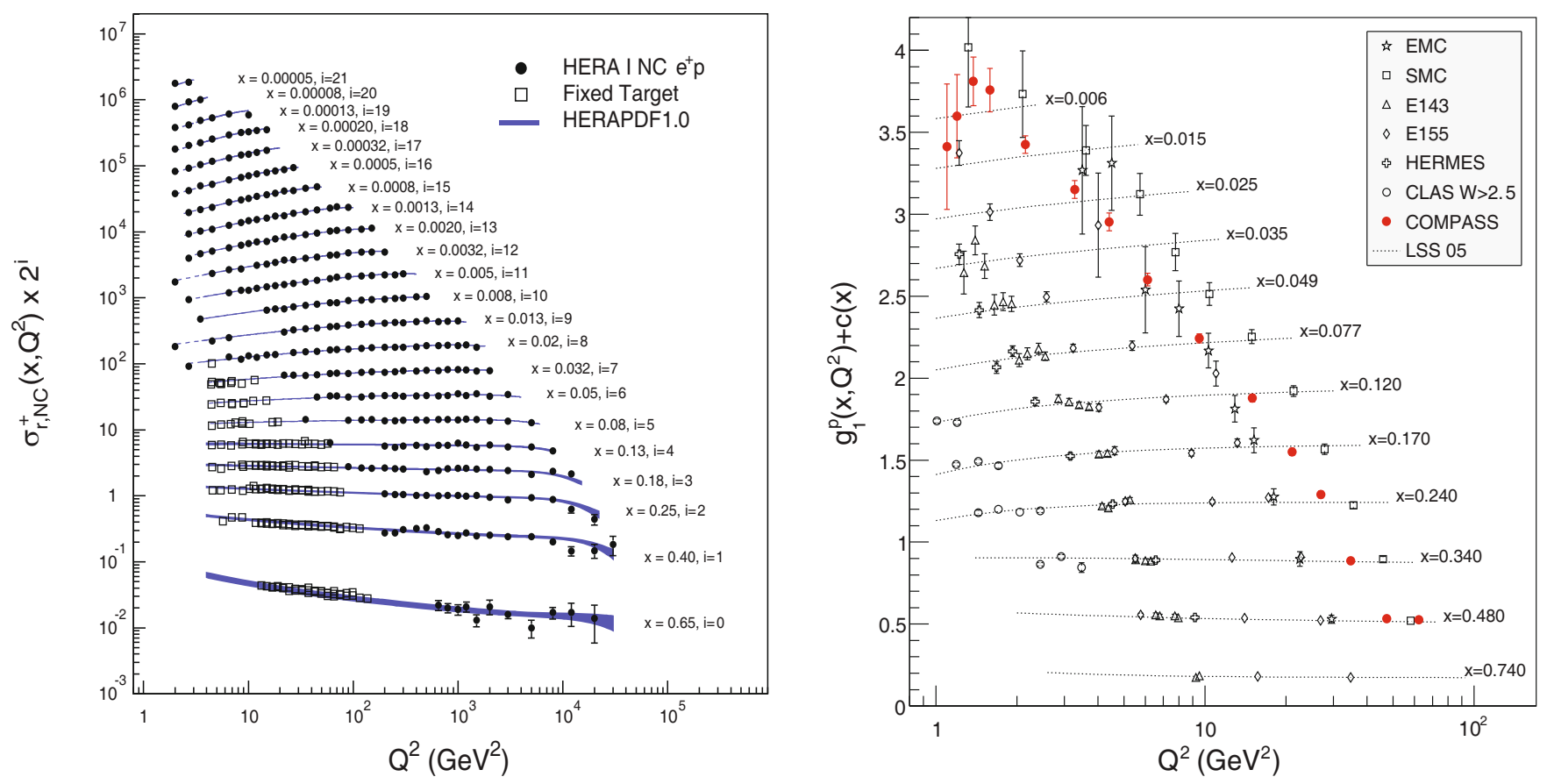

Fig. 8. Left: the ep reduced cross-section as measured at HERA and from fixed-target experiments as a function of $Q^{2}$ for fixed values of $x$ [10]. The data are compared to a pQCD fit. Right: the spin-dependent structure function $g_{1}\left(x, Q^{2}\right)$ as a function of $x$ and $Q^{2}[11]$. The world data are compared to a pQCD fit.

the polarization difference of cross-sections when the lepton and the nucleon collide with their spins anti-aligned or aligned:

$$
\frac{1}{2}\left[\frac{\mathrm{d}^{2} \sigma^{\leftrightarrows}}{\mathrm{d} x \mathrm{~d} Q^{2}}-\frac{\mathrm{d}^{2} \sigma^{\rightrightarrows}}{\mathrm{d} x \mathrm{~d} Q^{2}}\right] \simeq \frac{4 \pi \alpha^{2}}{Q^{4}} y(2-y) g_{1}\left(x, Q^{2}\right) .
$$

The expression above assumes photon exchange between the lepton and the nucleon. At high energies, also $W$ or $Z$ exchange contribute and lead to additional structure functions. These have thus far not been accessible in polarized deep inelastic scattering experiments and would be a unique opportunity at an EIC. We will briefly address them below.

In leading order in the strong coupling constant, the structure function $g_{1}\left(x, Q^{2}\right)$ of the proton can be written as (see Sidebar II)

$$
g_{1}\left(x, Q^{2}\right)=\frac{1}{2} \sum e_{q}^{2}\left[\Delta q\left(x, Q^{2}\right)+\Delta \bar{q}\left(x, Q^{2}\right)\right]
$$

where $e_{q}$ denotes a quark's electric charge. Higherorder expansions contain calculable QCD coefficient functions $[12-14]$. The structure function $g_{1}\left(x, Q^{2}\right)$ is thus directly sensitive to the nucleon spin structure in terms of the combined quark and anti-quark spin degrees of freedom. The gluon distribution $\Delta g$ enters the expression for $g_{1}$ only at higher order in perturbation theory; however, it drives the scaling violations (i.e. the $Q^{2}$-dependence) of $g_{1}\left(x, Q^{2}\right)$. Deep inelastic measurements hence can also give insight into gluon polarization, provided a large lever arm in $Q^{2}$ is available at fixed $x$.

\subsubsection{Status and near term prospects}

The EMC experiment [5, 24], using a longitudinally polarized muon beam and a stationary target that contained polarized protons, was the first experiment to explore $g_{1}\left(x, Q^{2}\right)$ down to momentum fractions $x$ as low as 0.01 . When extrapolated over unmeasured $x<0.01$ and combined with the couplings in leptonic hyperon decays and the assumption of $S U(3)$ flavor symmetry [25, 26], this led to the famous conclusion that the quark and anti-quark spins constitute only a small fraction of the proton spin. In addition, with these assumptions, the polarization of the strange quark sea in the polarized proton is found to be negative. Significant progress has been made since the EMC observations on the proton's spin composition. One main focus has been on measurements with longitudinally polarized lepton beams scattering off longitudinally polarized nucleons in stationary targets. Inclusive data have been obtained in experiments at CERN [27-29], DESY [30,31], Jefferson Laboratory $[32,33]$, and SLAC [34-38] in scattering off targets with polarized protons and neutrons. The kinematic reach and precision of the data on $g_{1}\left(x, Q^{2}\right)$ so far is similar to that of the unpolarized structure function $F_{2}\left(x, Q^{2}\right)$ just prior to the experimental program at the HERA electronproton collider (cf. Sidebar II).

Figure 9 provides a survey of the regions in $x$ and $Q^{2}$ covered by the world polarized-DIS data, which is roughly $0.004<x<0.8$ for $Q^{2}>1 \mathrm{GeV}^{2}$. For a representative value of $x \simeq 0.03$, the $g_{1}\left(x, Q^{2}\right)$ data are in the range $1 \mathrm{GeV}^{2}<Q^{2}<10 \mathrm{GeV}^{2}$. This is to be compared to $1 \mathrm{GeV}^{2}<Q^{2}<2000 \mathrm{GeV}^{2}$ for the unpolarized data on 


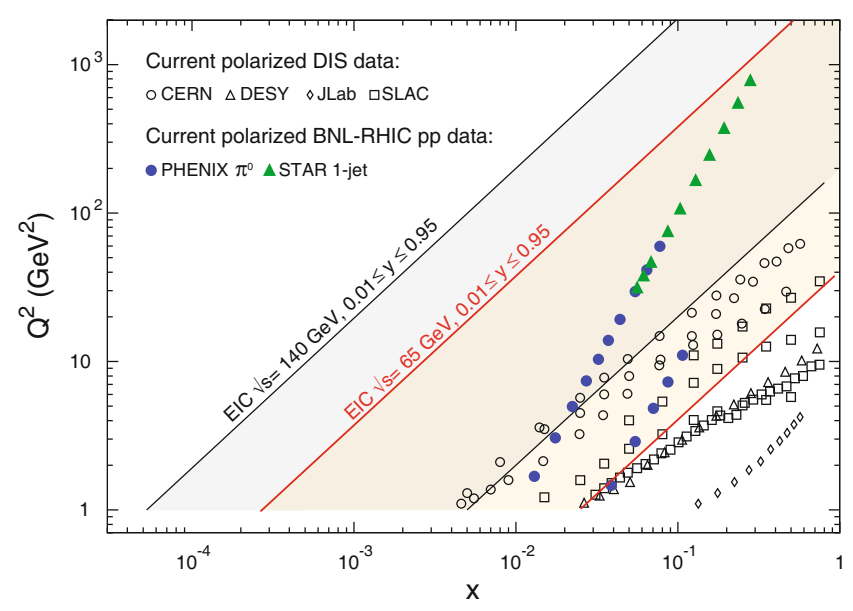

Fig. 9. Regions in $x, Q^{2}$ covered by previous spin experiments and anticipated to be accessible at an EIC. The values for the existing fixed-target DIS experiments are shown as data points. The RHIC data are shown at a scale $Q^{2}=p_{T}^{2}$, where $p_{T}$ is the observed jet (pion) transverse momentum, and an $x$ value that is representative for the measurement at that scale. The $x$ ranges probed at different scales are wide and have considerable overlap. The shaded regions show the $x, Q^{2}$ reach of an EIC for center-of-mass energy $\sqrt{s}=65 \mathrm{GeV}$ and $\sqrt{s}=140 \mathrm{GeV}$, respectively.

$F_{2}\left(x, Q^{2}\right)$ at the same $x$. The figure also shows the vast expansion in $x, Q^{2}$ reach that an EIC would provide, as will be discussed below. Over the past 15 years, an additional powerful line of experimental study of nucleon spin structure has emerged: semi-inclusive deep inelastic scattering. In these measurements, a charged or identified hadron $h$ is observed in addition to the scattered lepton. The relevant spin-dependent structure function,

$$
\begin{aligned}
g_{1}^{h}\left(x, Q^{2}, z\right)= & \frac{1}{2} \sum_{q} \cdot e_{q}^{2}\left[\Delta q\left(x, Q^{2}\right) D_{q}^{h}\left(z, Q^{2}\right)\right. \\
& \left.+\Delta \bar{q}\left(x, Q^{2}\right) D_{\bar{q}}^{h}\left(z, Q^{2}\right)\right]
\end{aligned}
$$

depends on fragmentation functions $D_{q, \bar{q}}^{h}\left(z, Q^{2}\right)$, where $z$ is the momentum fraction that is transferred from the outgoing quark or anti-quark to the observed hadron $h$. The non-perturbative fragmentation functions are at present determined primarily from precision data on hadron production in $e^{+} e^{-}$annihilation through perturbative QCD analysis [39-43]. Data from the $B$-factories and the LHC are helping to further improve their determination [43]. Also measurements of hadron multiplicities at an EIC would contribute to a better knowledge of fragmentation functions. Insights from the semi-inclusive measurements are complementary to those from the inclusive measurements. Specifically, they make it possible to delineate the quark and anti-quark spin contributions by flavor, since $\Delta q$ and $\Delta \bar{q}$ appear with different weights in eq. (11). A large body of semi-inclusive data sensitive to nucleon helicity structure has been collected by the experiments at CERN [44-46] and DESY [47].
A further milestone in the study of the nucleon was the advent of RHIC, the world's first polarized proton+ proton collider. In the context of the exploration of nucleon spin structure, the RHIC spin program is a logical continuation. Very much in the spirit of the unpolarized hadron colliders in the 1980 's, RHIC entered the scene to provide complementary information on the nucleon that is not readily available in fixed-target lepton scattering. The measurement of the spin-dependent gluon distribution $\Delta g\left(x, Q^{2}\right)$ in the proton is a major focus and strength of RHIC. Here the main tools are spin asymmetries in the production of inclusive pions [48-52] and jets [53-57] at large transverse momentum perpendicular to the beam axis, which sets the hard scale $Q$ in these reactions. Their reach in $x$ and $Q^{2}$ is also indicated in fig. 9. Unlike DIS, the processes used at RHIC do not probe the partons locally in $x$, but rather sample over a region in $x$. RHIC also provides complementary information on $\Delta u, \Delta \bar{u}, \Delta d, \Delta \bar{d}$ for $0.05<x<0.5$ [58-61], with a beautiful technique that exploits the violation of parity (mirror symmetry) in nature and does not rely on knowledge of fragmentation. The carriers of the charged-current weak interactions, the $W$ bosons, naturally select left-handed quarks and righthanded anti-quarks, and their production in $p+p$ collisions at RHIC and calculable leptonic decay hence provide an elegant probe of nucleon helicity structure.

Combined next-to-leading order QCD analyses [62-65] of the published data from inclusive and semi-inclusive deep inelastic scattering and from $p+p$ scattering at RHIC have been performed, which provide the best presently available information of the nucleon's helicity structure. The main results of the first such analysis $[62,63]$ are displayed in fig. 12. Here we describe the main qualitative features found in the latest studies:

- The combination of the large body of inclusive deep inelastic scattering data off targets containing polarized protons and neutrons has established that the up quarks and anti-quarks combine to have net polarization along the proton spin, whereas the down quarks and anti-quarks combine to carry negative polarization. The "total" $\Delta u+\Delta \bar{u}$ and $\Delta d+\Delta \bar{d}$ helicity distributions are very well constrained by now at medium to large $x$.

- The light sea quark and anti-quark distributions still carry large uncertainties, even though there are some constraints by the semi-inclusive data and, most recently, from measurements of spin-dependence in leptonic $W$ decay in $\sqrt{s}=500 \mathrm{GeV}$ polarized proton+proton collisions at RHIC $[60,61]$. RHIC probes the $\Delta u, \Delta d, \Delta \bar{u}$ and $\Delta \bar{d}$ densities for $0.05<x<0.5$ at a scale set by the $W$ mass [66]. The sea shows hints of not being $S U(2)$-flavor symmetric: the $\Delta \bar{u}$ distribution has a tendency to be mainly positive, while the $\Delta \bar{d}$ anti-quarks carry opposite polarization. This pattern has been predicted at least qualitatively by a number of models of the nucleon (for a review, see [67]). More sensitive constraints on $\Delta u, \Delta d, \Delta \bar{u}$ and $\Delta \bar{d}$ are anticipated [68] from additional RHIC measurements with higher integrated luminosity. The large luminosi- 
ties and high resolution available at the Jefferson Laboratory after an upgrade to $12 \mathrm{GeV}$ electron beam energy will extend the kinematic reach of the existing Jefferson Laboratory inclusive and semi-inclusive deep inelastic scattering data to twice smaller $x$ as well as to larger $x$ than have thus far been measured.

- Strange quarks appear to be deeply involved in nucleon spin structure. As we mentioned earlier, from the inclusive deep inelastic data, along with $S U(3)$ flavor symmetry considerations, one derives a strong negative value of the integrated strange helicity distribution. Strange quarks and anti-quarks would thus be polarized opposite to the nucleon. This would need to be viewed as part of the reason why the total quark and anti-quark spin contribution $S_{q}$ is so much smaller than expected in simple models. On the other hand, significant $S U(3)$ flavor symmetry breaking effects have been discussed in the literature [69-73]. The semiinclusive measurements with identified kaons $[46,47]$ are hence of particular interest since they yield the most direct and best sensitivity thus far to the polarization of strange quarks and anti-quarks, albeit with considerable dependence on the kaon fragmentation functions [74]. No evidence for sizable $\Delta s\left(x, Q^{2}\right)$ or $\Delta \bar{s}\left(x, Q^{2}\right)$ has been found in polarized semi-inclusive measurements with fixed targets. As a consequence, $\Delta s$ would need to obtain its negative integral purely from the contribution from the thus far unmeasured small- $x$ region. This exemplifies the need for simultaneous measurements of the kaon production crosssections and their spin-dependence in semi-inclusive deep inelastic scattering at smaller $x$.

- Constraints on the spin-dependent gluon distribution $\Delta g$ predominantly come from RHIC, with some information also entering from scaling violations of the deep inelastic structure function $g_{1}\left(x, Q^{2}\right)$. The production cross sections for inclusive hadrons and jets at RHIC receive contributions from gluon-gluon and quark-gluon scattering and probe $\Delta g\left(x, Q^{2}\right)$ over the range $0.02<x<0.4$. Note that the $x$ is not explicitly resolved in measurements of inclusive pion and jet probes. Initial results from RHIC saw small doublespin asymmetries for inclusive jets and hadrons. As a result, the global analysis $[62,63]$ concluded that there were no indications of a sizable contribution of gluon spins to the proton spin. This has changed recently: The latest much more precise STAR results for the double-spin asymmetry in jet production [57] provide, for the first time, evidence of a non-vanishing polarization of gluons in the nucleon in the RHIC kinematic regime [64]. This is a major breakthrough for this field. The limited $x$-range and unresolved $x$-dependence preclude definitive conclusions on the total gluon spin contribution to the proton spin, $S_{g}$, although it appears likely now that gluons are an important player for the proton spin. Continued measurements at $\sqrt{s}=$ $200 \mathrm{GeV}$ will enhance the sensitivity primarily at large $x$, and measurements of correlated probes are anticipated to yield insights in $x$-dependence. Forthcoming measurements at $\sqrt{s}=500 \mathrm{GeV}$ are expected to ex- tend the small- $x$ reach to $2-3$ times smaller values and modest further gains may be possible with new instruments at larger pseudorapidity. Extrapolation over the unmeasured $x \lesssim 0.01$ region is precarious, and definitive resolution of the gluon spin contribution to the nucleon spin thus relies on a new generation of experiments.

\subsubsection{Open questions and the role of an EIC}

The overarching scientific question - How is the spin of the proton distributed among its quark and gluon constituents? - will remain only partially answered even after the completion of the present programs and their upgrades. Concerning the helicity parton distributions, the remaining key open issues will be:

- What is the gluon spin contribution to the proton spin? As we saw, there is now initial knowledge about $\Delta g$ in a relatively narrow region of $x$. Clearly, more extended coverage is required to determine this intrinsic property of the proton and constrain the integral of the distribution.

- What polarization is carried by the proton's light sea? Previous and present experiments give a hint at the interesting flavor structure of sea quark polarization. Still, even after the completion of the RHIC program with $W$ bosons, we will likely have little precision on, for example $\Delta \bar{u}-\Delta \bar{d}$, a quantity that features prominently in virtually all models of the nucleon in ways that are complementary to the unpolarized light sea. Exploring in detail the proton's sea quark "landscape" would provide unprecedented insight into nonperturbative QCD.

- What role do strange quarks play in nucleon spin structure? Strange quarks play a special role for understanding QCD as their mass is of the order of $\Lambda_{\mathrm{QCD}}$ and they are hence to be considered neither light (as the up and down quarks), nor heavy (as the charm and heavier quarks). The present experimental information on their role in nucleon spin structure is quite puzzling, as we described above. There is clearly a strong need to determine $\Delta s$ and $\Delta \bar{s}$ over a wide range in $x$. This will also probe important aspects of $S U(3)$ flavor symmetry and its breaking in QCD.

In order to fully solve the proton spin problem one evidently also needs to obtain information on quark and gluon orbital angular momenta in the nucleon. This requires a new suite of measurements, using exclusive processes such as deeply virtual Compton scattering and transverse-spin asymmetries. The associated physics and the prospects of measurements at an EIC will be described in sects. 2.3 and 2.4 .

The envisioned polarized electron ion collider brings unique capabilities to the study of nucleon spin. Its high center-of-mass energy of up to $\sqrt{s}=173 \mathrm{GeV}$ affords access to a vast region in $x$ and $Q^{2}$ that will probe $1-2$ orders of magnitude smaller values in $x$ than the body of existing and forthcoming data and comparably harder scales $Q^{2}$, 
Table 1. Key measurements to determine the quark and gluon helicity distributions in the polarized nucleon.

\begin{tabular}{|c|c|c|c|}
\hline Deliverables & Observables & What we learn & Requirements \\
\hline \hline $\begin{array}{c}\text { polarized gluon } \\
\text { distribution } \Delta g\end{array}$ & $\begin{array}{c}\text { scaling violations } \\
\text { in inclusive DIS }\end{array}$ & $\begin{array}{c}\text { gluon contribution } \\
\text { to proton spin }\end{array}$ & $\begin{array}{c}\text { coverage down to } x \simeq 10^{-4} ; \\
\mathcal{L} \text { of about } 10 \mathrm{fb}^{-1}\end{array}$ \\
\hline $\begin{array}{c}\text { polarized quark and } \\
\text { antiquark densities }\end{array}$ & $\begin{array}{c}\text { semi-incl. DIS for } \\
\text { pions and kaons }\end{array}$ & $\begin{array}{c}\text { quark contr. to proton spin; } \\
\text { asym. like } \Delta \bar{u}-\Delta \bar{d} ; \Delta s\end{array}$ & $\begin{array}{c}\text { similar to DIS; } \\
\text { good particle ID }\end{array}$ \\
\hline $\begin{array}{c}\text { novel electroweak } \\
\text { spin structure functions }\end{array}$ & inclusive DIS & flavor separation & $\sqrt{s} \geq 100 \mathrm{GeV} ; \mathcal{L} \geq 10 \mathrm{fb}^{-1}$ \\
positrons; polarized $d$ or $^{3} \mathrm{He}$ beam
\end{tabular}

as is clearly visible from fig. 9. The high luminosity and polarization will allow one to do so with precision using a suite of probes (see table 1). In this way, the EIC aims to provide answers to the questions raised above.

We will now discuss the scientific highlights of an EIC, insofar as they pertain to nucleon helicity structure.

Arguably the golden "flagship" measurement of nucleon spin structure at the EIC will be a precision study of the proton's spin structure function $g_{1}\left(x, Q^{2}\right)$ and its scaling violations, over wide ranges in $x$ and $Q^{2}$. The methods to measure $g_{1}\left(x, Q^{2}\right)$ are well known experimentally and $g_{1}\left(x, Q^{2}\right)$ is also understood very well theoretically. The small- $x$ region is key to determining and understanding the role of sea quarks and gluons in the spin decomposition of the nucleon. The structure function $g_{1}\left(x, Q^{2}\right)$ presently is terra incognita for $x<0.004$ and $Q^{2}>1 \mathrm{GeV}^{2}$ (see fig. 9). Low- $x$ measurements of $g_{1}$ reduce the present uncertainty associated with the required extrapolation when computing the quark and anti-quark spin contribution $S_{q}$ to the proton spin. The $Q^{2}$-dependence of $g_{1}\left(x, Q^{2}\right)$ will give unprecedented insight into gluon polarization. The EIC will also vastly expand our knowledge of the quark flavor structure, a key element in mapping out the proton "landscape". A powerful measurement available to achieve this is semi-inclusive deep inleastic scattering which at the EIC would extend to much higher $Q^{2}$ than in fixed-target scattering, where the interpretation becomes significantly cleaner, less afflicted by corrections suppressed by $1 / Q^{2}$, and better tractable theoretically. The kinematic coverage in $x$ and $Q^{2}$ will be similar to what can be achieved in inclusive scattering.

To illustrate the tremendous impact of EIC measurements of inclusive and semi-inclusive polarized deep inelastic scattering on our knowledge of helicity parton distributions, a series of perturbative QCD analyses was performed [75] with realistic pseudo-data for various centerof-mass energies. The data simulations were based on the PEPSI Monte Carlo generator [76]. The precision of the data sets corresponds to an accumulated integrated luminosity of $10 \mathrm{fb}^{-1}$ (or one to two months of running for most energies at the anticipated luminosities) and an assumed operations efficiency of $50 \%$. A minimum $Q^{2}$ of $1 \mathrm{GeV}^{2}$ was imposed, as well as $W^{2}>10 \mathrm{GeV}^{2}$, a depolarization factor of the virtual photon of $D(y)>0.1$, and $0.01 \leq y \leq 0.95$. Figure 10 shows the pseudo-data for the inclusive structure function $g_{1}\left(x, Q^{2}\right)$ of the proton versus $Q^{2}$ at fixed $x$.

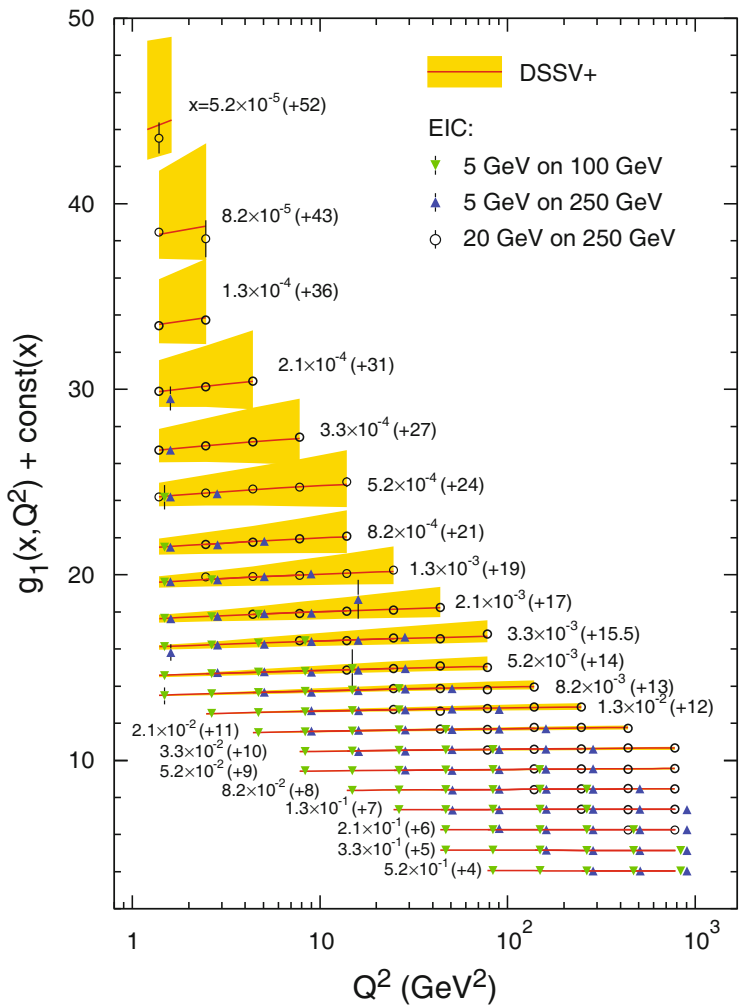

Fig. 10. EIC pseudo-data on the inclusive spin structure function $g_{1}\left(x, Q^{2}\right)$ versus $Q^{2}$ at fixed $x$ for $5 \mathrm{GeV}$ and $20 \mathrm{GeV}$ electron beams colliding with $100 \mathrm{GeV}$ and $250 \mathrm{GeV}$ proton beam energies at an EIC, as indicated. The error bars indicate the size of the statistical uncertainties. The data set for each $x$ is offset by a constant $c(x)$ for better visibility. The bands indicate the current uncertainty as estimated in the "DSSV+" analysis (see text).

Collisions at $\sqrt{s} \simeq 70 \mathrm{GeV}$ at an EIC are seen to provide access to $x$ values down to about $2 \times 10^{-4}$. The anticipated size of the asymmetry $A_{1}\left(x, Q^{2}\right) \simeq$ $g_{1}\left(x, Q^{2}\right) / F_{1}\left(x, Q^{2}\right)$ at these $x$ values is $\mathcal{O}\left(10^{-3}\right)$, which sets the scale for the required data samples and control of experiment systematics. These and other aspects are discussed further in sect. 6. Data from a higher-energy EIC, shown for electron beam energies up to $20 \mathrm{GeV}$, is seen to provide access to significantly smaller $x$ and larger $Q^{2}$. As demonstrated in fig. 11, the combination of measurements with a wide range of center-of-mass energies 


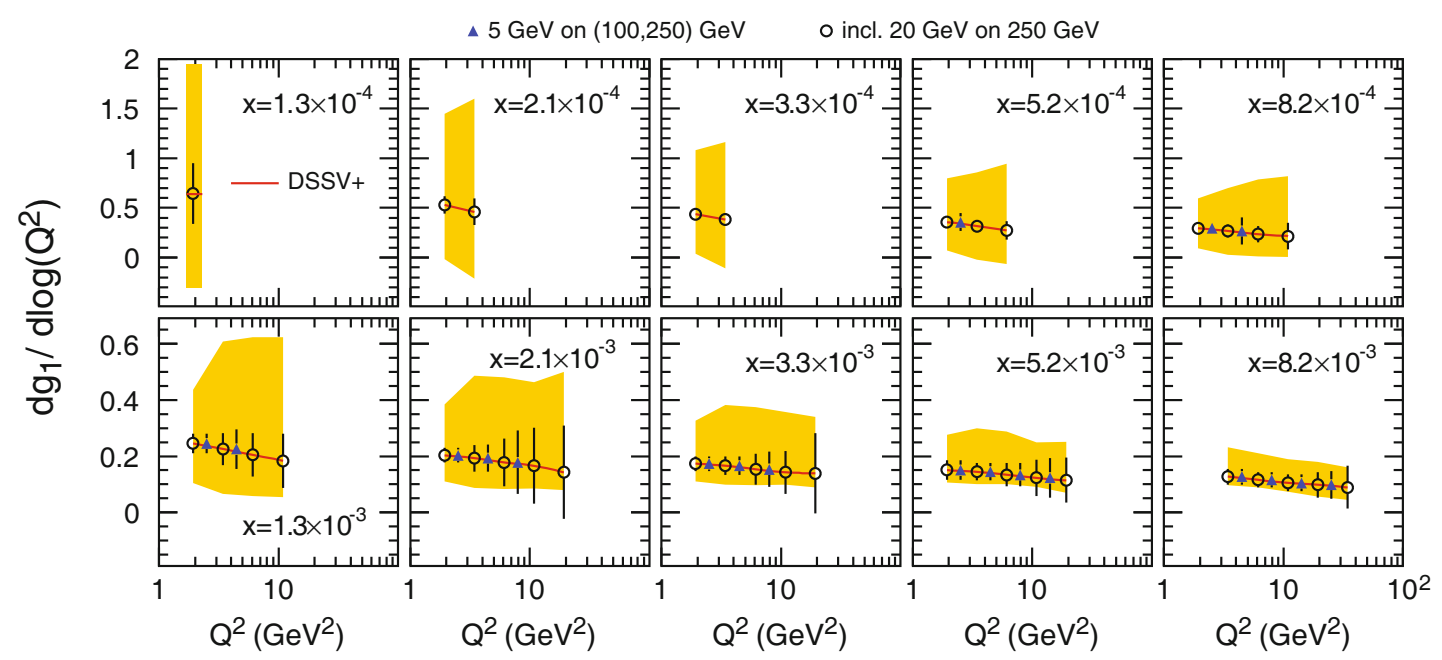

Fig. 11. The derivative of $g_{1}\left(x, Q^{2}\right)$ with $\log \left(Q^{2}\right)$ for different $x$ values for the same combination of electron and proton beam energies as used in fig. 10, together with the "DSSV+" uncertainty bands.
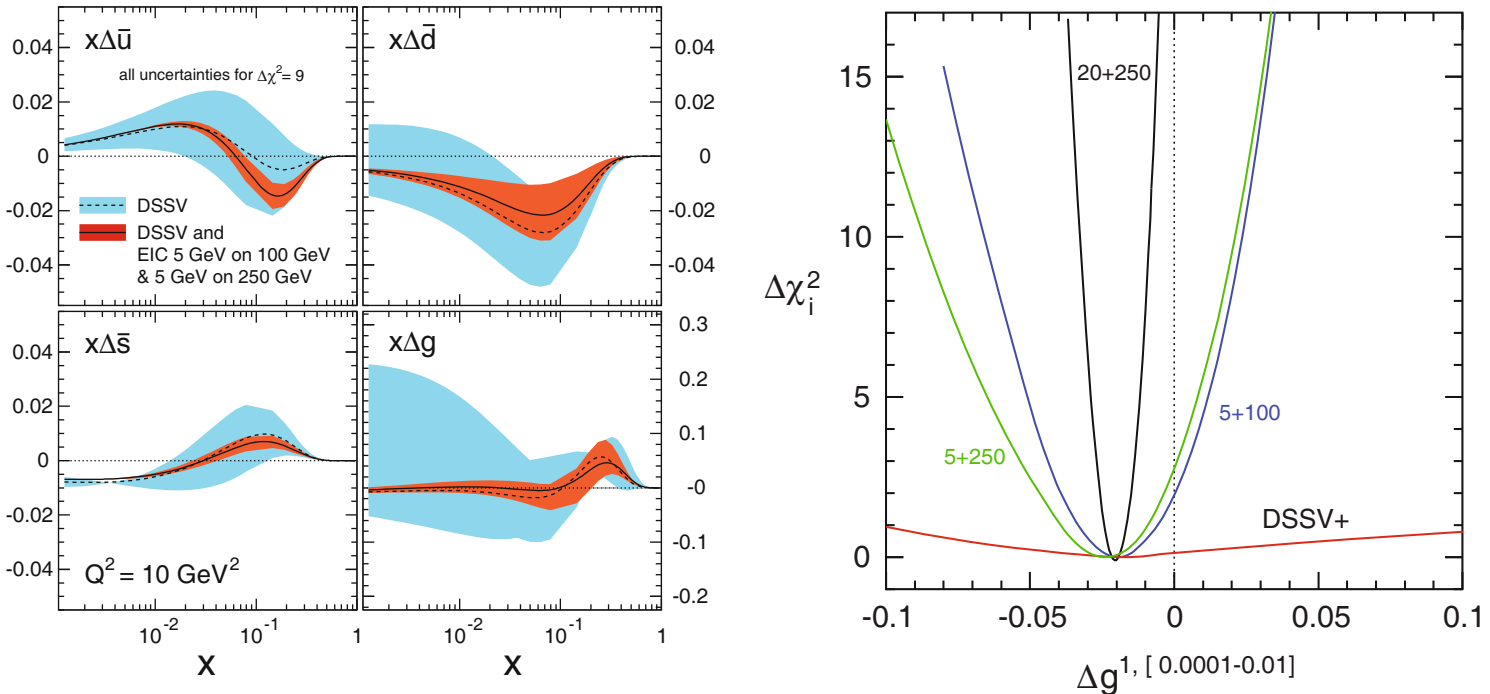

Fig. 12. Left: uncertainty bands on helicity parton distributions, in the first DSSV analysis [62, 63] (light bands) and with EIC data (darker bands), using projected inclusive and semi-inclusive EIC data sets (see text). Note that for this analysis only data with $x \geq 10^{-3}$ were used, for which $Q^{2} \geq 2.5 \mathrm{GeV}^{2}$. Right: $\chi^{2}$ profiles for the truncated $x$ integral of $\Delta g$ over the region $10^{-4} \leq x \leq 10^{-2}$ with and without including the generated EIC pseudo-data in the fit. Results are shown for three different EIC center-of-mass energies.

at an EIC will make it possible to directly determine $\mathrm{d} g_{1}\left(x, Q^{2}\right) / \mathrm{d} \log \left(Q^{2}\right)$ with good sensitivity, which directly probes the gluon distribution $\Delta g$.

The pseudo-data for $g_{1}$ and for semi-inclusive spin asymmetries were included [75] in the global analysis of helicity-dependent parton distribution functions based on the DSSV framework $[62,63]^{2}$. Figure 12 (left) shows the results of this analysis in terms of the sea quark and gluon

\footnotetext{
2 As described earlier, these first DSSV papers do not yet contain the latest information from RHIC on $\Delta g$, which were not yet available at the time of [75]. However, this is not an issue here as the figures below are merely meant to demonstrate the improvements an EIC would provide on the knowledge of the helicity distributions. We note that for the studies presented here the analysis of $[62,63]$ has been complemented
}

helicity distributions. For comparison, the present uncertainty bands are also displayed. As one can see, an impressive reduction in the width of the bands would be expected from EIC data, in particular, towards lower values of $x$. Evidently, extractions of $\Delta g$ from scaling violations, and of the light-flavor helicity distributions $\Delta u$, $\Delta d$ and their anti-quark distributions from semi-inclusive scattering will be possible with exquisite precision. With dedicated studies of kaon production, the strange and antistrange distributions will also be accessible. All this is anticipated to yield new insights into the question of why it is that the combined quark and anti-quark spin contribution to the proton spin turns out to be so small.

with recent lepton scattering data $[29,46]$ from CERN. It will henceforth be referred to as "DSSV+" analysis. 


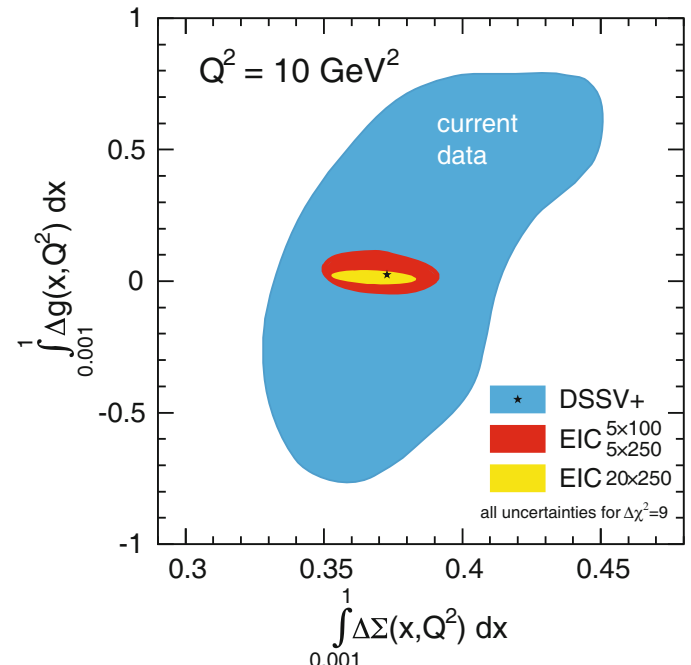

Fig. 13. Accuracies for the correlated truncated integrals of $\Delta \Sigma$ and $\Delta g$ over $0.001 \leq x \leq 1$, on the basis of the "DSSV+" analysis (outer area) and projected for an EIC (inner areas) [75].

The right part of the figure shows the $\chi^{2}$ profile of the truncated first moment of the gluon helicity distribution, $\int_{0.0001}^{0.01} \mathrm{~d} x \Delta g\left(x, Q^{2}\right)$, at $Q^{2}=10 \mathrm{GeV}^{2}$, again compared to the "DSSV+" estimate. Also here, the impact of EIC data is evident. One also observes the importance of high energies. For instance, running at the highest energy clearly constrains the small- $x$ region much better. Overall, the EIC data greatly improves the $\chi^{2}$ profile, even more so when all data in fig. 10 are included.

The light shaded area in fig. 13 displays the present accuracies of the integrals of $\Delta \Sigma$ and $\Delta g$ over $0.001 \leq x \leq 1$, along with their correlations. The inner areas represent the improvement to be obtained from the EIC, based on the global analysis studies with pseudo-data described above. We stress that similar relative improvements would occur for any other benchmark set of polarized parton distribution functions, such as the latest DSSV [64] set. The results shown in the figure clearly highlight the power of an EIC in mapping out nucleon helicity structure. The anticipated kinematic range and precision of EIC data will give unprecedented insight into the spin contributions $S_{q}$ and $S_{g}$. Their measurements, by subtracting from the total proton spin $1 / 2$, will provide stringent and independent constraints on the total contribution of quark and gluon orbital momenta, $L_{q}+L_{g}$.

Besides polarized proton beams, the EIC design envisions beams of polarized deuterons or helium-3. The neutron's $g_{1}\left(x, Q^{2}\right)$ can thus be determined, potentially with a precision that is comparable to the data on $g_{1}\left(x, Q^{2}\right)$ of the proton. The difference of the moments of proton and neutron $g_{1}\left(x, Q^{2}\right)$ allows a test of the fundamental sum rule by Bjorken [77]. The data from polarized fixed-target experiments have verified the sum rule to a precision of about $10 \%$ of its value. The extended kinematic range and improved precision of EIC data allow for more stringent tests of this sum rule, as well as its corrections, to an ac-

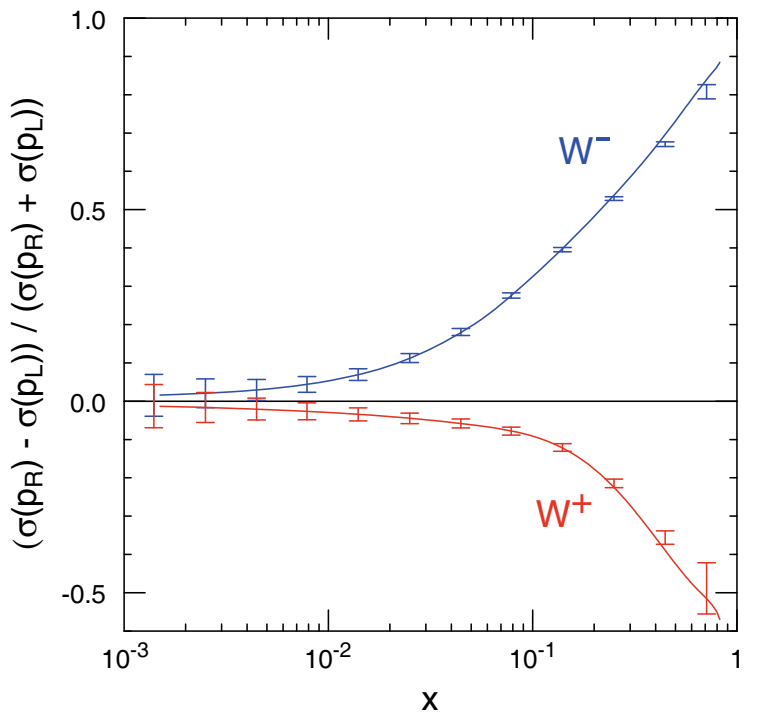

Fig. 14. Single-longitudinal-spin asymmetries for $W^{-}$and $W^{+}$exchange at an EIC, using polarized protons. A collision energy of $\sqrt{s}=141 \mathrm{GeV}$ was assumed and cuts $Q^{2}>1 \mathrm{GeV}^{2}$ and $0.1<y<0.9$ were applied. The uncertainties shown are statistical, for $10 \mathrm{fb}^{-1}$ integrated luminosity.

curacy that is currently anticipated to be driven mostly by advances in hadron beam polarimetry (cf. sect. 6.3.2).

An additional, and unique, avenue for delineating the flavor structure of the quark and anti-quark spin contribution to the proton spin at the EIC is electroweak deep inelastic scattering. At high $Q^{2}$, the deep inelastic process also proceeds significantly via exchange of $Z$ and $W^{ \pm}$ bosons. This gives rise to novel structure functions that are sensitive to different combinations of the proton's helicity distributions. For instance, in the case of charged-current interactions through $W^{-}$, the inclusive structure functions contribute,

$$
\begin{aligned}
& g_{1}^{W^{-}}\left(x, Q^{2}\right)=[\Delta u+\Delta \bar{d}+\Delta c+\Delta \bar{s}]\left(x, Q^{2}\right), \\
& g_{5}^{W^{-}}\left(x, Q^{2}\right)=[-\Delta u+\Delta \bar{d}-\Delta c+\Delta \bar{s}]\left(x, Q^{2}\right),
\end{aligned}
$$

where $\Delta c$ denotes the proton's polarized charm quark distribution. The analysis of these structure functions does not rely on knowledge of fragmentation. Studies show that both neutral-current and charged-current interactions would be observable at the EIC, even with relatively modest integrated luminosities. To fully exploit the potential of the EIC for such measurements, positron beams are required, albeit not necessarily polarized. Besides the new insights into nucleon structure this would provide, studies of spin-dependent electroweak scattering at short distances with an EIC would be beautiful physics in itself, much in the line of past and ongoing electroweak measurements at HERA, Jefferson Laboratory, RHIC, and the LHC.

As an illustration of the EIC's potential in this area, fig. 14 shows production level estimates for chargedcurrent interactions through $W^{-}$and $W^{+}$exchange at collision energy $\sqrt{s}=141 \mathrm{GeV}$. Cuts of $Q^{2}>1 \mathrm{GeV}^{2}$ 


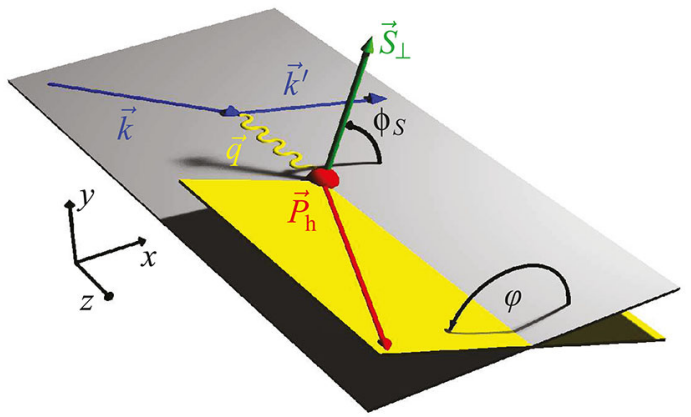

Fig. 15. Semi-inclusive hadron production in DIS processes: $e+N \rightarrow e^{\prime}+h+X$, in the target rest frame. $\boldsymbol{P}_{h T}$ and $\boldsymbol{S}_{\perp}$ are the transverse components of $\boldsymbol{P}_{h}$ and $\boldsymbol{S}$ with respect to the virtual photon momentum $\boldsymbol{q}=\boldsymbol{k}-\boldsymbol{k}^{\prime}$.

and $0.1<y<0.9$ have been applied. The figure shows the parity-violating single-longitudinal-spin asymmetry $\left(\sigma\left(p_{R}\right)-\sigma\left(p_{L}\right)\right) /\left(\sigma\left(p_{R}\right)+\sigma\left(p_{L}\right)\right)$ obtained from the crosssections for positive $\left(p_{R}\right)$ or negative $\left(p_{L}\right)$ proton helicity. The figure also shows production level statistical uncertainties for measurements at an EIC with $10 \mathrm{fb}^{-1}$ integrated luminosity. As one can see, large asymmetries are expected in the region of moderate-to-large $x$, where the energies of the observed jet are typically large. Their measurement provides unique insights into the flavor composition of the proton spin. A more detailed study has recently been published [78].

\section{Sidebar III. Semi-inclusive Deep Inelastic Scattering}

Semi-inclusive hadron production in deep inelastic scattering (SIDIS) (fig. 15) provides a powerful probe of the transverse-momentum-dependent (TMD) quark distributions of nucleons. Common kinematic variables have been described in the DIS section (see Sidebar I). In SIDIS, the kinematics of the final-state hadrons can be specified as follows:

$\phi_{h}, \phi_{s}$ : azimuthal angles of the final-state hadron and the transverse polarization vector of the nucleon with respect to the lepton plane.

$P_{h T}$ : transverse momentum of the final-state hadron with respect to the virtual photon in the center-ofmass of the virtual photon and the nucleon.

$z=P_{h} \cdot P / q \cdot P$ gives the momentum fraction of the final-state hadron with respect to the virtual photon.

The differential SIDIS cross section can be written as a convolution of the transverse-momentum-dependent quark distributions $f\left(x, k_{T}\right)$, fragmentation functions $D\left(z, p_{T}\right)$, and a factor for a quark or antiquark to scatter off the photon. At the leading power of $1 / Q$, we can probe eight different TMD quark distributions as listed in fig. 16. These distributions represent various correlations between the transverse momentum of the quark $\boldsymbol{k}_{T}$, the nucleon momentum $\boldsymbol{P}$, the nucleon spin $\boldsymbol{S}$, and the quark $\operatorname{spin} \boldsymbol{s}_{q}$.

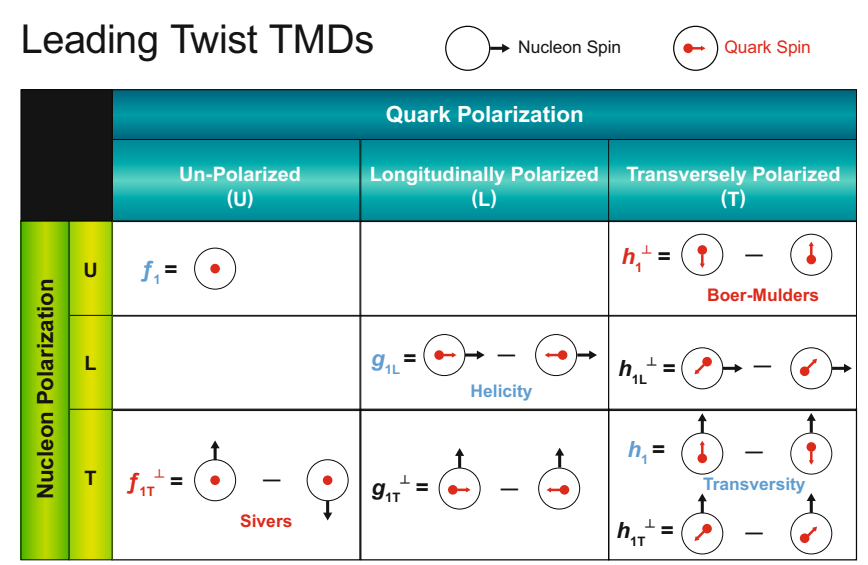

Fig. 16. Leading twist TMDs classified according to the polarizations of the quark $(f, g, h)$ and nucleon $(U, L, T)$. The distributions $f_{1 T}^{\perp, q}$ and $h_{1}^{\perp, q}$ are called naive-time-reversal-odd TMDs. For gluons a similar classification of TMDs exists.

\subsection{Confined motion of partons in nucleons: TMDs ${ }^{3}$}

\subsubsection{Introduction}

DIS is a powerful way to probe the internal structure of nucleons. After four decades of experiments scattering high-energy leptons off nucleons, our knowledge of the nucleon structure has made impressive progress. However, our understanding of the nucleon structure from inclusive DIS experiments is basically one-dimensional. From inclusive DIS we "only" learn about the longitudinal motion of partons in a fast-moving nucleon, whose transverse momenta are not resolved. Meanwhile, the past decade has witnessed tremendous experimental achievements which led to fascinating new insights into the structure of the nucleon through semi-inclusive hadron production in DIS (SIDIS) and hard exclusive processes in DIS. These less inclusive methods enable us to investigate the partonic structure of the nucleon beyond one-dimensional space. As discussed at the beginning of this section, these developments have stimulated theoretical advances from a simple parton model description of nucleon structure to multi-dimensional distributions of partons, including the generalized parton distributions (GPDs), the transversemomentum-dependent parton distributions (TMDs), and the quantum phase space Wigner distributions. The focus of this section is on the TMDs, their theoretical properties and phenomenological implications, and the experimental access to them. TMDs open a new window to understand some of the most fundamental aspects of QCD. Several fascinating topics are related to the study of TMDs:

- 3D-imaging. The TMDs represent the intrinsic motion of partons inside the nucleon (confined motion!) and allow reconstruction of the nucleon structure in momentum space. Such information, when combined with the analogous information on the parton spatial distribution from GPDs, leads to a 3-dimensional imaging of the nucleon.

\footnotetext{
${ }^{3}$ Conveners: Haiyan Gao and Feng Yuan.
} 
- Orbital motion. Most TMDs would vanish in the absence of parton orbital angular momentum, and thus enable us to quantify the amount of orbital motion.

- Spin-orbit correlations. Most TMDs and related observables are due to couplings of the transverse momentum of quarks with the spin of the nucleon (or the quark). Spin-orbit correlations in QCD, akin to those in hydrogen atoms and topological insulators, can therefore be studied.

- Gauge invariance and universality. The origin of some TMDs and related spin asymmetries, at the partonic level, depend on fundamental properties of QCD, such as its color gauge invariance. This leads to clear differences between TMDs in different processes, which can be experimentally tested.

The "simplest" TMD is the unpolarized function $f_{1}^{q}\left(x, k_{T}\right)$, which describes, in a fast moving nucleon, the probability of finding a quark carrying the longitudinal momentum fraction $x$ of the nucleon momentum, and a transverse momentum $k_{T}=\left|\boldsymbol{k}_{T}\right|$. It is related to the collinear ("integrated") PDF by $\int \mathrm{d}^{2} \boldsymbol{k}_{T} f_{1}^{q}\left(x, k_{T}\right)=$ $f_{1}^{q}(x)$. In addition to $f_{1}^{q}\left(x, k_{T}\right)$, there are two other TMDs: $g_{1 L}^{q}\left(x, k_{T}\right)$ and $h_{1}^{q}\left(x, k_{T}\right)$, whose integrals correspond to the collinear PDFs: the longitudinal polarized structure function discussed in the previous section and the quark transversity distribution. The latter is related to the tensor charge of the nucleon. These three distributions can be regarded as a simple transverse-momentum extension of the associated integrated quark distributions. More importantly, the power and rich possibilities of the TMD approach arise from the simple fact that $\boldsymbol{k}_{T}$ is a vector, which allows for various correlations with the other vectors involved: the nucleon momentum $\boldsymbol{P}$, the nucleon spin $\boldsymbol{S}$, and the parton spin (say a quark, $\boldsymbol{s}_{q}$ ). Accordingly, there are eight independent TMD quark distributions as shown in fig. 16. Apart from the straightforward extension of the normal PDFs to the TMDs, there are five TMD quark distributions, which are sensitive to the direction of $k_{T}$, and will vanish with a simple $k_{T}$ integral.

Because of the correlations between the quark transverse momentum and the nucleon spin, the TMDs naturally provide important information on the dynamics of partons in the transverse plane in momentum space, as compared to the GPDs which describe the dynamics of partons in the transverse plane in position space. Measurements of the TMD quark distributions provide information about the correlation between the quark orbital angular momentum and the nucleon/quark spin because they require wave function components with nonzero orbital angular momentum. Combining the wealth of information from all of these functions could thus be invaluable for disentangling spin-orbit correlations in the nucleon wave function, and providing important information about the quark orbital angular momentum. One particular example is the quark Sivers function $f_{1 T}^{\perp q}$ which describes the transverse-momentum distribution correlated with the transverse polarization vector of the nucleon. As a result, the quark distribution will be azimuthally asymmetric in the transverse-momentum space in a trans-

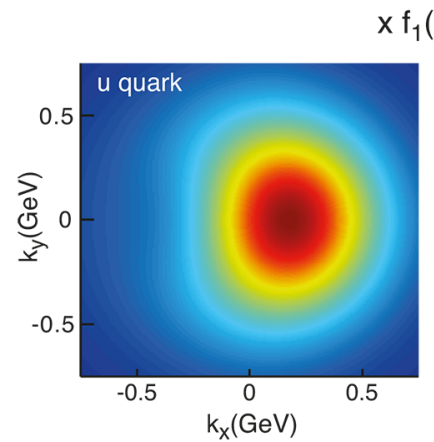

$$
x f_{1}\left(x, k_{T}, S_{T}\right)
$$

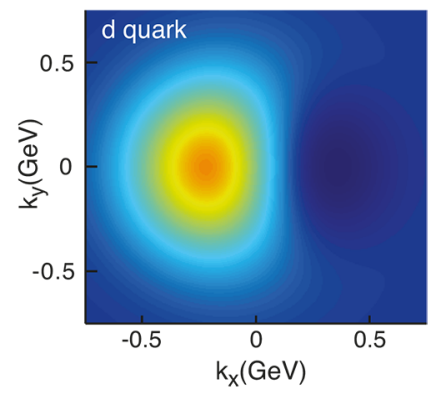

Fig. 17. The density in the transverse-momentum plane for unpolarized quarks with $x=0.1$ in a nucleon polarized along the $\hat{y}$ direction. The anisotropy due to the proton polarization is described by the Sivers function, for which the model of [79] is used. The deep red (blue) indicates large negative (positive) values for the Sivers function.

versely polarized nucleon. Figure 17 demonstrates the deformations of the up and down quark distributions. There is strong evidence of the Sivers effect in the DIS experiments observed by the HERMES, COMPASS, and JLab Hall A collaborations [80-82]. An important aspect of the Sivers functions that has been revealed theoretically in last few years is the process dependence and the color gauge invariance [83-86]. Together with the Boer-Mulders function, they are denoted as naive time-reversal odd ( $T$-odd) functions. In SIDIS, where a leading hadron is detected in coincidence with the scattered lepton, the quark Sivers function arises due to the exchange of (infinitely many) gluons between the active struck quark and the remnants of the target, which is referred to as final-state interaction effects in DIS. On the other hand, for the Drell-Yan lepton pair production process, it is due to the initial-state interaction effects. As a consequence, the quark Sivers and Boer-Mulders functions differ by a sign in these two processes. This non-universality is a fundamental prediction from the gauge invariance of QCD [84]. The experimental check of this sign change is currently one of the outstanding topics in hadronic physics, and Sivers functions from the Drell-Yan process can be measured at RHIC.

\subsubsection{Opportunities for measurements of TMDs at the EIC}

To study the transverse-momentum-dependent parton distributions in high-energy hadronic processes, an additional hard momentum scale is essential, besides the transverse momentum, for proper interpretation of results. This hard momentum scale needs to be much larger than the transverse momentum. At the EIC, DIS processes naturally provide a hard momentum scale: $Q$, the virtuality of the photon. More importantly, the wide range of $Q^{2}$ values presents a unique opportunity to systematically investigate the strong interaction dynamics associated with the TMDs. Although there has been tremendous progress in understanding TMDs, without a new lepton-hadron collider, many aspects of TMDs will remain unexplored - or 
at best be explored only on a qualitative level. Existing facilities either suffer from a much too restricted kinematic coverage or from low luminosity or from both.

The SIDIS measurement discussed below is the necessary method to access TMDs. We define two planes in SIDIS: the lepton plane and the hadron plane, as shown in fig. 15, which allows us to study different angular dependences in the hadron production cross-sections. These angular distributions are important to extract the TMDs since each of them has a unique angular dependence. Precision measurements of the various angular modulations are only possible with a comprehensive and hermetic detector. With such a detector and the EIC's ability to provide a wide kinematic range and high luminosity, we see the following opportunities for measurements at an EIC that would be impossible in current experiments:

- high precision quantitative measurements of all the quark TMDs in the valence region, with the ability to go to sufficiently large values of $Q^{2}$ in order to suppress potential higher twist contaminations;

- first-ever measurements of the TMDs for anti-quarks and gluons;

- multi-dimensional representations of the observables leading to TMDs;

- systematic studies of perturbative QCD techniques (for polarization observables) and studies of QCD evolution properties of TMDs;

- the transition between the non-perturbative low transverse-momentum region and perturbative high transverse-momentum region for both polarized and unpolarized collisions due to a wide range of kinematic coverage.

The above discussions apply to all of the eight TMD quark distributions listed in fig. 16. The rich physics covered by the TMD quark and gluon distribution functions can be thoroughly investigated at the EIC with a dedicated detector. In the following subsections, we will take semi-inclusive DIS as an example for the quark Sivers function and di-hadron production for the gluon Sivers function and highlight the impact the EIC could have on these measurements.

\section{Semi-inclusive Deep Inelastic Scattering}

The TMDs are measured using SIDIS processes. In such reactions, the hadron, which results from the fragmentation of a scattered quark, "remembers" the original motion of the quark, including its transverse momentum. SIDIS depends on six kinematic variables. In addition to the variables for inclusive DIS, $x, y=(P \cdot q) /(P \cdot l)$, and the azimuthal angle $\phi_{S}$ describing the orientation of the target spin vector for transverse polarization, one has three variables for the final state hadron, which we denote by $z=\left(P \cdot P_{h}\right) /(P \cdot q)$ (longitudinal hadron momentum fraction), $P_{h T}$ (magnitude of transverse hadron momentum), and the angle $\phi_{h}$ for the orientation of $\boldsymbol{P}_{h T}$ (see fig. 15). In the one-photon exchange approximation, the SIDIS crosssection can be decomposed in terms of structure functions.

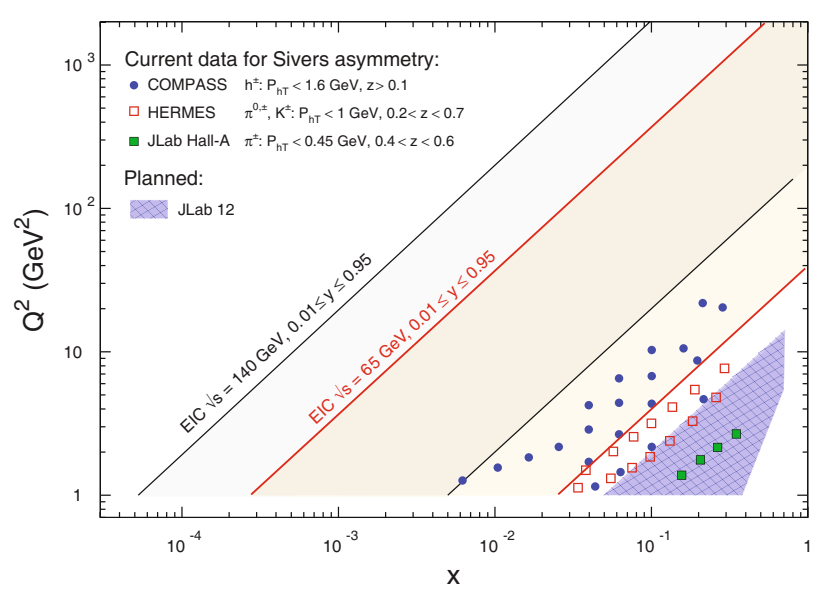

Fig. 18. Kinematic coverage in $x$ and $Q^{2}$ for the EIC compared to the coverage of the planned JLab12 experiment. The kinematics of the existing experimental measurements are also shown for comparison.

Each of them is characterized by the unique azimuthal angular modulation in the differential cross-sections. The extraction of these structure functions will give access to all of the leading TMD quark distributions listed in fig. 16.

For example, for the spin-average and single-spindependent contributions, we have

$$
\begin{aligned}
& \frac{\mathrm{d} \sigma}{\mathrm{d} x \mathrm{~d} y \mathrm{~d} \phi_{S} \mathrm{~d} z \mathrm{~d} \phi_{h} \mathrm{~d} P_{h T}^{2}} \propto \\
& F_{U U, T}+\left|\boldsymbol{S}_{\perp}\right| \sin \left(\phi_{h}-\phi_{S}\right) F_{U T, T}^{\sin \left(\phi_{h}-\phi_{S}\right)}+\ldots,
\end{aligned}
$$

where $F_{U U}$ represents the spin-average structure function depending on the unpolarized quark distribution $f_{1}^{q}\left(x, k_{T}\right)$, and $F_{U T}$ depends on the quark Sivers function $f_{1 T}^{\perp q}\left(x, k_{T}\right)$. For TMD studies, one is interested in the kinematic region defined by $P_{h T} \ll Q$, for which the structure functions can be written as certain convolutions of TMDs. To extract the quark Sivers function, we measure the $\sin \left(\phi_{h}-\phi_{s}\right)$ modulation of the single-transverse-spin asymmetry (SSA), which is defined by the ratio of the two cross-section terms in eq. (13). This asymmetry depends on four kinematics: $Q^{2}, x_{B}, z_{h}, P_{h T}$. A systematic and detailed study of the Sivers function, and TMDs in general, can only be performed on the basis of precise spin and azimuthal asymmetry amplitude measurements in SIDIS over a wide kinematic range. In fig. 18, we compare the $x-Q^{2}$ coverage of the HERMES, COMPASS, and JLab $12 \mathrm{GeV}$ upgrade with the coverage of an EIC. The wide kinematic coverage puts the EIC in the unique position of accessing the valence region at much larger $Q^{2}$ than current and near-future experiments while also accessing low- $x$ down to values of about $10^{-5}$, where sea quarks and gluons could be studied in detail. The expected high luminosity will also allow for a fully differential analysis over almost the entire kinematic range of $x, Q^{2}, z$ and $P_{h T}$, which is vital for phenomenological analyses. 


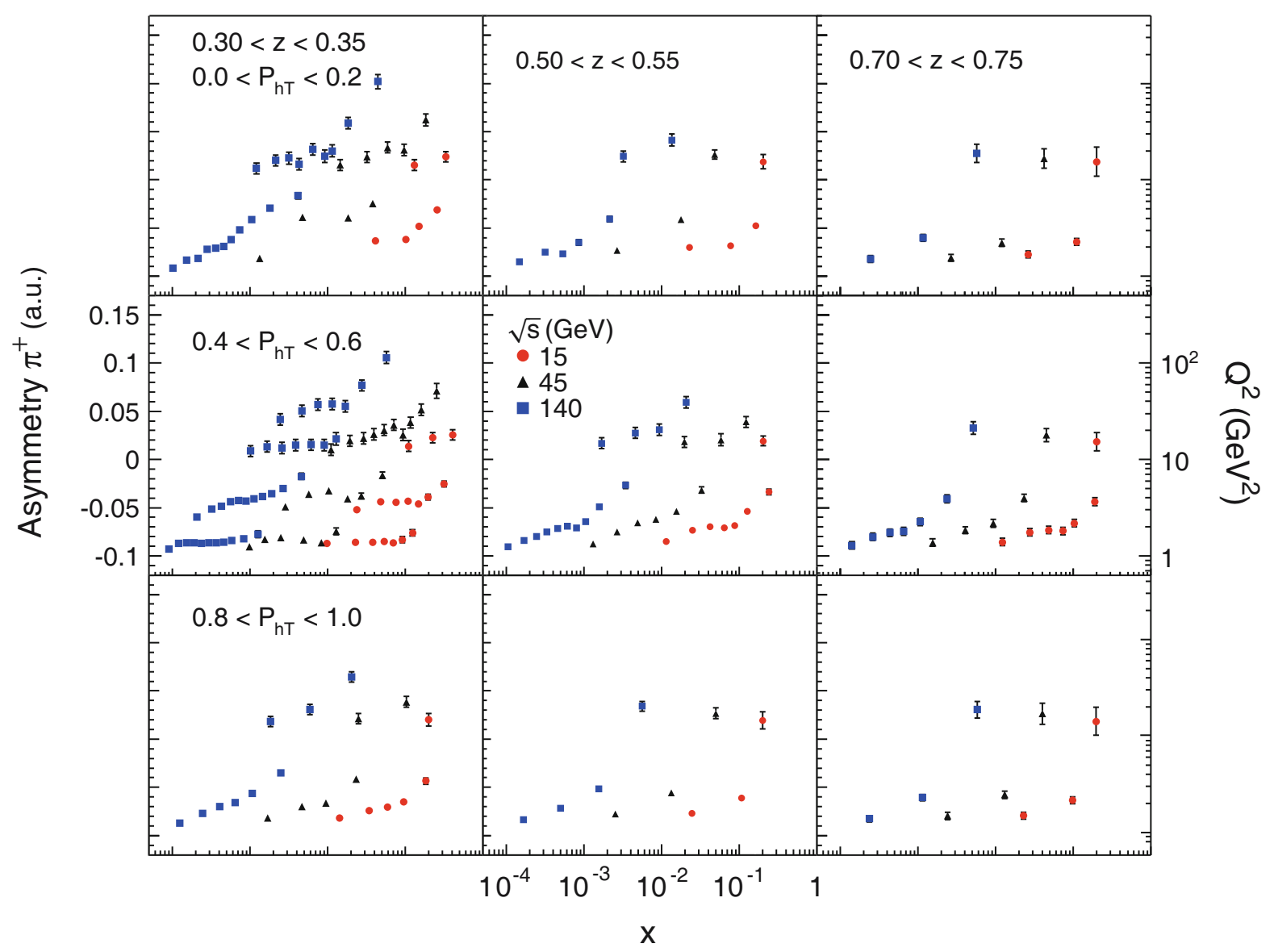

Fig. 19. Four-dimensional representation of the projected accuracy for $\pi^{+}$production in semi-inclusive DIS off the proton. Each panel corresponds to a specific $z$ bin with increasing value from left to right and a specific $P_{h T}$ bin with increasing value from top to bottom, with values given in the figure. The position of each point is according to its $Q^{2}$ and $x$ value, within the range $0.05<y<0.9$. The projected event rate, represented by the error bar, is scaled to the (arbitrarily chosen) asymmetry value at the right axis. Blue squares, black triangles and red dots represent the $\sqrt{s}=140 \mathrm{GeV}, \sqrt{s}=45 \mathrm{GeV}$ and $\sqrt{s}=15 \mathrm{GeV}$ EIC configurations, respectively. Event counts correspond to an integrated luminosity of $10 \mathrm{fb}^{-1}$ for each of the three configurations.

In the following, we illustrate the expected impact of data from the EIC using the parameterization from ref. [79] as an arbitrarily chosen model of the Sivers function. This parameterization, denoted theor ${ }_{i}=F\left(x_{i}, z_{i}\right.$, $\left.P_{h T}^{i}, Q_{i}^{2} ; \mathbf{a}_{0}\right)$ with the $M$ parameters $\mathbf{a}_{0}=\left\{a_{1}^{0}, \ldots, a_{M}^{0}\right\}$ fitted to existing data, serves to generate a set of pseudodata in each kinematic bin $i$. In each $x_{i}, Q_{i}^{2}, z_{i}$ and $P_{h T}^{i}$ bin, the obtained values, value $_{i}$, for the Sivers function are distributed using a Gaussian smearing with a width $\sigma_{i}$ corresponding to the simulated event rate at the center-ofmass energy of $\sqrt{s}=45 \mathrm{GeV}$ obtained with an integrated luminosity of $10 \mathrm{fb}^{-1}$. To illustrate the achievable statistical precision, the event rate for the production of $\pi^{ \pm}$in semi-inclusive DIS was used, see, for example, fig. 19.

This new set of pseudo-data was then analysed like the real data in ref. [79]. Figure 20 shows the result for the extraction of the Sivers function for the valence and sea up quarks. Similar results are obtained for the down quarks as well. The central value of $f_{1 T}^{\perp u}$, represented by the red line, follows by construction the underlying model. The 2-sigma uncertainty of this extraction, valid for the specifically chosen functional form, is indicated by the pur- ple band. This precision, obtainable with an integrated luminosity of $10 \mathrm{fb}^{-1}$, is compared with the uncertainty of the extraction from existing data, represented by the light grey band. It should be emphasized that our current knowledge is restricted to only a qualitative picture of the Sivers function and the above analysis did not take into account the model dependence and the associated theoretical uncertainties. With the anticipated large amount of data (see fig. 19 for a modest integrated luminosity $10 \mathrm{fb}^{-1}$ ), we can clearly see that the EIC will be a powerful facility enabling access to TMDs with unprecedented precision, and particularly in the currently unexplored sea quark region. This precision is not only crucial for the fundamental QCD test of the sign change between the Sivers asymmetries in the DIS and Drell-Yan processes, but also important to investigate the QCD dynamics in the hard processes in SIDIS, such as the QCD evolution and resummation, matching between the TMD factorization and collinear factorization approaches, etc. Meanwhile, an exploration of the sea quark Sivers function will provide, for the first time, unique information on the spin-orbital correlation in the small- $x$ region. 

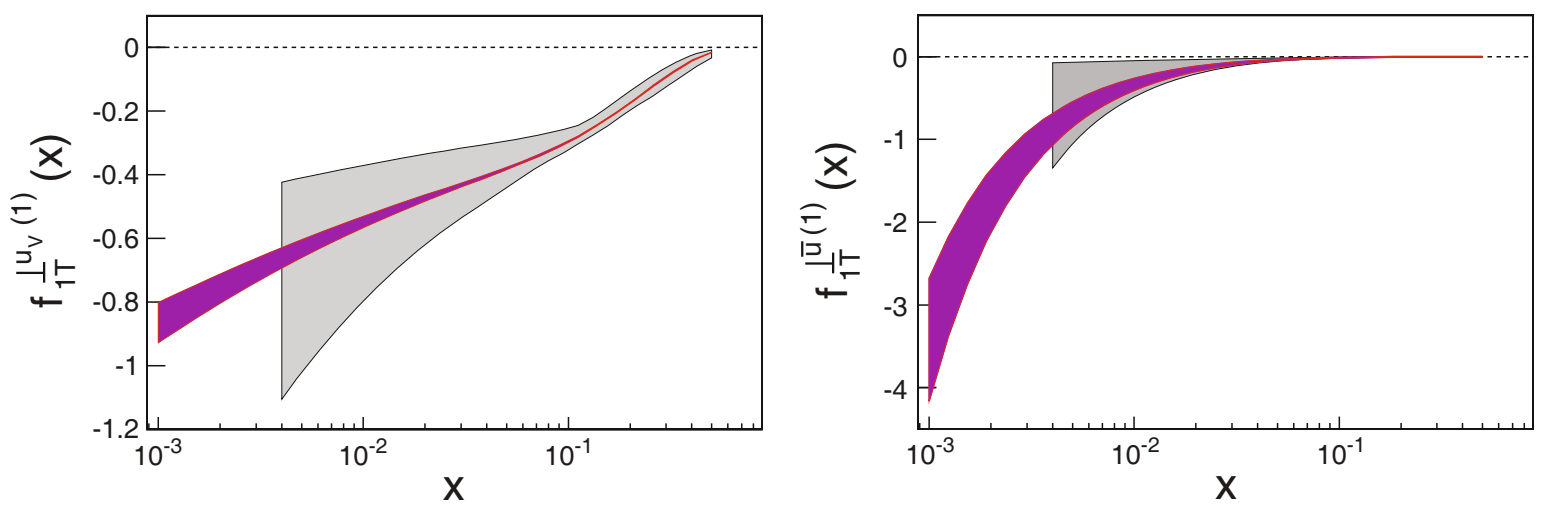

Fig. 20. Comparison of the precision ( $2 \sigma$ uncertainty) of extractions of the Sivers function for the valence (left) $u_{v}=u-\bar{u}$ and sea (right) $\bar{u}$ quarks from currently available data [79] (grey band) and from pseudo-data generated for the EIC with energy setting of $\sqrt{s}=45 \mathrm{GeV}$ and an integrated luminosity of $10 \mathrm{fb}^{-1}$ (purple band with a red contour). The uncertainty estimates are for the specifically chosen underlying functional form.

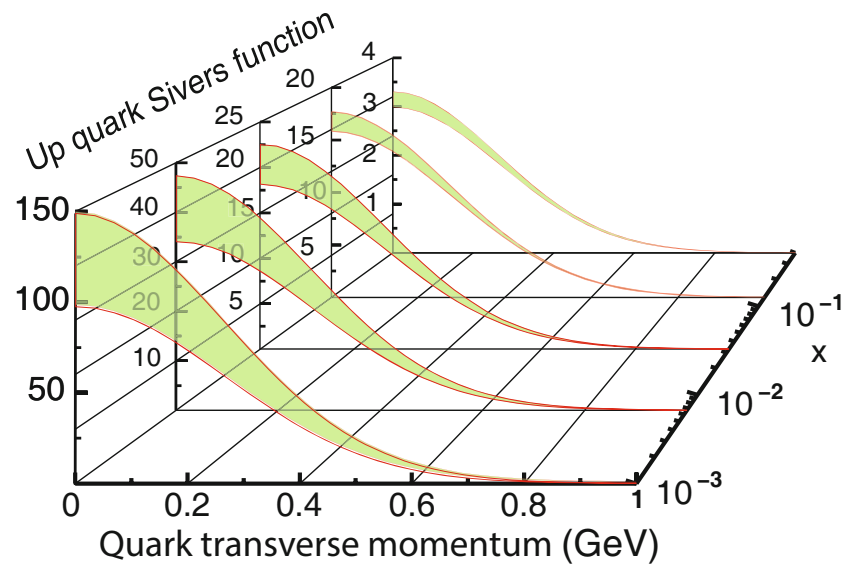

Fig. 21. The transverse-momentum profile of the up quark Sivers function at five $x$ values accessible to the EIC, and corresponding statistical uncertainties.

Figure 21 showed the kinematic reach of the EIC which would enable a measurement of the transverse-momentum profile of the quark Sivers function over a wide range in $x$, e.g. from the valence to the sea quark region. Note that fig. 21 showed the total up quark Sivers function, while fig. 20 shows the valence and the sea quarks separately.

Here, we emphasize the importance of the high $Q^{2}$ reach of the EIC for SIDIS measurements. Most of the existing experiments focus on the $Q^{2}$ range of a few $\mathrm{GeV}^{2}$. The EIC will, for the first time, reach $Q^{2}$ values up to hundreds and more $\mathrm{GeV}^{2}$. This will provide an unique opportunity to investigate the scale evolution of the Sivers asymmetries, which has attracted strong theoretical interests in the last few years [87-92]. As a leading power contribution in the spin asymmetries, the associated energy evolution unveils the underlying strong interaction dynamics in the hard scattering processes. The embedded universality and factorization property of the TMDs can only be fully investigated at the EIC with the planned kinematic coverage in $Q^{2}$. In particular, the theory calculations including evolution effects agree with the cur- rent constraints on the quark Sivers function presented in fig. 21, while they do differ at higher values of $Q^{2}$ [87-92]. Moreover, a recent study has shown that at the kinematics of HERMES and COMPASS, the leading-order SIDIS suffers significant power corrections, which however will diminish at higher $Q^{2}$ [92]. This makes the EIC the only machine to be able to establish the leading partonic picture of the TMDs in SIDIS.

The kinematic reach of the EIC also allows the measurement of physical observables over a wide transversemomentum range. This is particularly important to understand the underlying mechanism that results in singlespin asymmetries. Recent theoretical developments have revealed that both the transverse-momentum-dependent Sivers mechanism and the quark-gluon-quark correlation collinear mechanism describe the same physics in the kinematic regions where both approaches apply [93,94]. The only way to distinguish between the two and understand the underlying physics is to measure them over wide $p_{T}$ ranges. The high luminosities at the EIC machine could provide a golden opportunity to explore and understand the mechanism of the transverse-spin asymmetries. In addition, with precision data in a large range of transverse momentum, we shall be able to study the strong interaction dynamics in the description of large-transversemomentum observables and investigate the transition between the non-perturbative low-transverse-momentum region and the perturbative high-transverse-momentum region.

\section{Access to the gluon TMDs}

Beyond the gluon helicity measurements described in sect. 2.2, the gluonic orbital angular momentum contribution would be studied in hard exclusive meson production processes at the EIC. The transverse-momentumdependent gluon distribution can provide complementary information on the spin-orbital correlation for the gluons in the nucleon. Just as there are eight TMDs for quarks, there exist eight TMDs for gluons [95]. Experimentally, the gluon TMDs -in particular, the gluon 


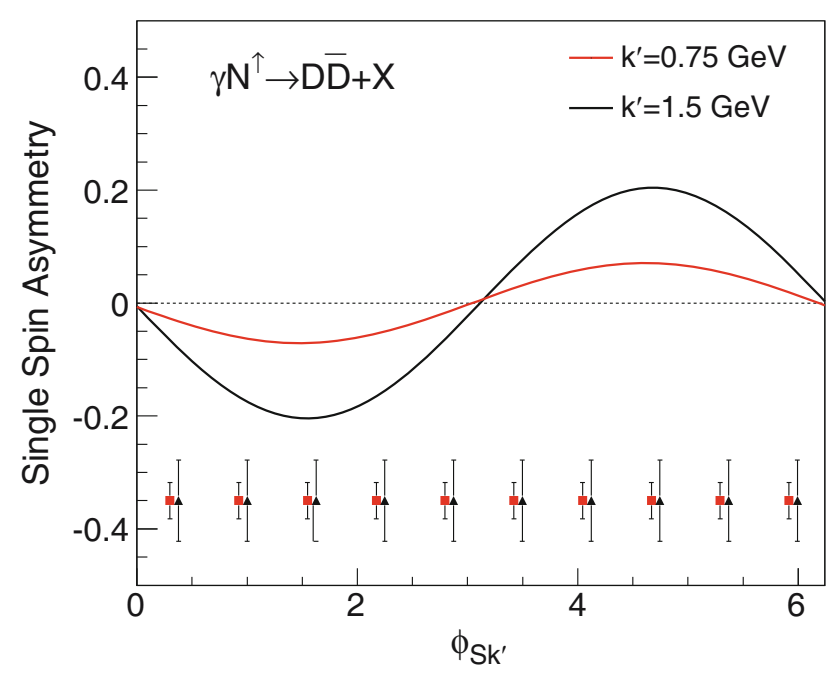

Fig. 22. The single-transverse-spin asymmetry for $\gamma^{*} N^{\uparrow} \rightarrow$ $D^{0} \bar{D}^{0}+X$, where $\phi$ is the azimuthal angle between the total transverse momentum $k_{\perp}^{\prime}$ of the $D-\bar{D}$ pair and the transverse polarization vector $S_{\perp}$ of the nucleon. The asymmetries and the experimental projections are calculated for two different $k_{\perp}^{\prime}=0.75,1.5 \mathrm{GeV}$ as examples. The kinematics are specified by $\langle W\rangle=60 \mathrm{GeV},\left\langle Q^{2}\right\rangle=4 \mathrm{GeV}^{2}$.

Sivers function - are completely unexplored so far and will likely not be probed at existing facilities. In addition, toward the small- $x$ region, the TMD gluon distributions have intimate connections to the saturation phenomena discussed in sect. 3.2, where the gluon distributions are fundamental objects as well. Explorations of the TMD gluon distributions (experimentally and theoretically) shall offer deep insight into the QCD dynamics evolving from the valence region to the sea region.

Many processes in DIS can be used to probe the transverse-momentum-dependent gluon distributions, for example, di-jet/di-hadron production, heavy-quark, and quarkonium production. We take one particular example: heavy-meson pair $(D-\bar{D})$ production in DIS. In this process, $D$ and $\bar{D}$ are produced in the current fragmentation region: $\gamma^{*} N^{\uparrow} \rightarrow D\left(k_{1}\right)+\bar{D}\left(k_{2}\right)+X$, where $N$ represents the transversely polarized nucleon, $D$ and $\bar{D}$ are the two mesons with momenta $k_{1}$ and $k_{2}$, respectively. Similar to the Sivers effect in semi-inclusive hadron production in DIS discussed above, the gluon Sivers function will introduce an azimuthal asymmetry correlating the total transverse momentum $k_{\perp}^{\prime}=k_{1 \perp}+k_{2 \perp}$ of the $D-\bar{D}$ pair with the transverse polarization vector $S_{\perp}$ of the nucleon. In experiment, this results in a single spin azimuthal asymmetry depending on the azimuthal angle between $k_{\perp}^{\prime}$ and $S_{\perp}$. In fig. 22, we show the sensitivity of the measurement of the asymmetry in a typical kinematic configuration of the EIC machine [96]. The two theory curves are based on a model calculation from ref. [2]. The estimate of the projected error bars comes from a simulation of the integrated luminosity of $100 \mathrm{fb}^{-1}$. Since the gluon Sivers effects has never been measured, this will be the first measurement of such an effect. Beside the $D-\bar{D}$ correlation, the di-hadron/di-jet correlations in DIS can also give us an independent handle on the study of the gluon Sivers function.

\subsubsection{Summary}

The EIC will be a unique facility to systematically investigate the transverse-momentum-dependent parton distributions comprehensively. While the measurements of quark TMDs have begun in fixed target experiments, the gluon TMDs can only be studied at an EIC, and such studies would be unprecedented. The QCD dynamics associated with the transverse-momentum dependence in hard processes can be rigorously studied at the EIC because of its wide kinematic coverage. The comparison of the Sivers single-spin asymmetry and Boer-Mulders asymmetry between DIS and Drell-Yan processes can provide an important test of the fundamental prediction of QCD. In summary, we list these important science questions to be addressed at the EIC in table 2.

\section{Sidebar IV. Exclusive processes and general- ized parton distributions}

Generalized parton distributions (GPDs) can be extracted from suitable exclusive scattering processes in $e+p$ collisions. Examples are deeply virtual Compton scattering (DVCS: $\gamma^{*}+p \rightarrow \gamma+p$ ) and the production of a vector meson $\left(\gamma^{*}+p \rightarrow V+p\right)$. The virtual photon is provided by the electron beam, as usual in deep inelastic scattering processes (see Sidebar I). GDPs depend on three kinematical variables and a resolution scale:

$-x+\xi$ and $x-\xi$ are longitudinal parton momentum fractions with respect to the average proton momentum $\left(p+p^{\prime}\right) / 2$ before and after the scattering, as shown in fig. 23 .

Whereas $x$ is integrated over in the scattering amplitude, $\xi$ is fixed by the process kinematics. For DVCS one has $\xi=x_{B} /\left(2-x_{B}\right)$ in terms of the usual Bjorken variable $x_{B}=Q^{2} /(2 p \cdot q)$. For the production of a meson with mass $M_{V}$ one finds instead $\xi=x_{V} /\left(2-x_{V}\right)$ with $x_{V}=\left(Q^{2}+M_{V}^{2}\right) /(2 p \cdot q)$.

- The crucial kinematic variable for parton imaging is the transverse momentum transfer $\boldsymbol{\Delta}_{T}=\boldsymbol{p}_{T}^{\prime}-\boldsymbol{p}_{T}$ to the proton. It is related to the invariant square $t=$ $\left(p^{\prime}-p\right)^{2}$ of the momentum transfer by $t=-\left(\Delta_{T}^{2}+\right.$ $\left.4 \xi^{2} M^{2}\right) /\left(1-\xi^{2}\right)$, where $M$ is the proton mass.

- The resolution scale is given by $Q^{2}$ in DVCS and lightmeson production, whereas for the production of a heavy meson such as the $J / \psi$ it is $M_{J / \psi}^{2}+Q^{2}$.

Even for unpolarized partons, one has a nontrivial spin structure, parameterized by two functions for each parton type. $H(x, \xi, t)$ is relevant for the case where the helicity of the proton is the same before and after the scattering, whereas $E(x, \xi, t)$ describes a proton helicity flip. For equal proton four-momenta, $p=p^{\prime}$, the distributions $H(x, 0,0)$ reduce to the familiar quark, anti-quark and gluon densities measured in inclusive processes, whereas the forward limit $E(x, 0,0)$ is unknown. 
Table 2. Science Matrix for TMD: 3D structure in transverse-momentum space: the golden measurements (upper part); the silver measurements (lower part).

\begin{tabular}{|c|c|c|}
\hline Deliverables & Observables & What we learn \\
\hline \hline Sivers \& & SIDIS with & Quantum interference \& spin-orbital correlations \\
unpolarized & transverse & 3D Imaging of quark's motion: valence + sea \\
TMD quarks & polarization; & 3D Imaging of gluon's motion \\
and gluon & di-hadron (di-jet) & QCD dynamics in an unprecedented $Q^{2}\left(P_{h T}\right)$ range \\
\hline \hline Chiral-odd & SIDIS with & 3rd basic quark PDF: valence + sea, tensor charge \\
functions: & transverse & Novel spin-dependent hadronization effect \\
transversity; & polarization & QCD dynamics in a chiral-odd sector \\
Boer-Mulders & & with a wide $Q^{2}\left(P_{h T}\right)$ coverage \\
\hline
\end{tabular}
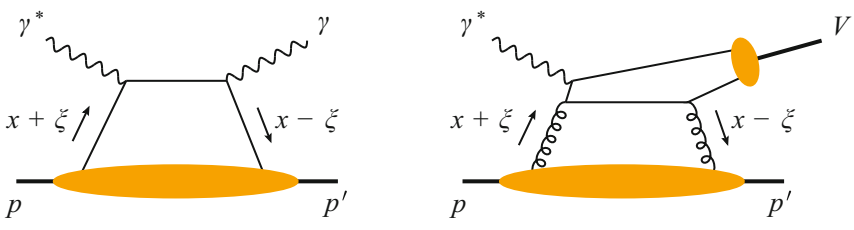

Fig. 23. Graphs for deeply virtual Compton scattering (left) and for exclusive vector meson production (right) in terms of generalized parton distributions, which are represented by the lower blobs. The upper filled oval in the right figure represents the meson wave function.

Weighting with the fractional quark charges $e_{q}$ and integrating over $x$, one obtains a relation with the electromagnetic Dirac and Pauli form factors of the proton:

$$
\begin{aligned}
& \sum_{q} e_{q} \int \mathrm{d} x H^{q}(x, \xi, t)=F_{1}^{p}(t), \\
& \sum_{q} e_{q} \int \mathrm{d} x E^{q}(x, \xi, t)=F_{2}^{p}(t)
\end{aligned}
$$

and an analogous relation to the neutron form factors. At small $t$ the Pauli form factors of the proton and the neutron are both large, so that the distributions $E$ for up and down quarks cannot be small everywhere.

\subsection{Spatial imaging of quarks and gluons ${ }^{4}$}

\subsubsection{Physics motivations and measurement principle}

\section{Spatial imaging}

Elastic electron-nucleon scattering has played a major role in our understanding of strong interactions ever since the Hofstadter experiment showed that protons and neutrons are not point-like particles. Measurements of the electromagnetic nucleon form factors have become ever more precise [97] and give detailed information about the spatial distribution of electric charge and magnetization

\footnotetext{
${ }^{4}$ Conveners: Markus Diehl and Franck Sabatié.
}

in the nucleon. Further information (albeit with less accuracy) can be obtained from neutral and charged weak currents. However, elastic scattering does not reveal the distribution of gluons, which carry only color charge, and it is not selectively sensitive to sea quarks.

Hard exclusive scattering processes bring the idea of imaging to a new qualitative level by probing the transverse distribution of quarks, anti-quarks and gluons as a function of their longitudinal momentum in the nucleon. One may regard this as a tomography of the nucleon, with two-dimensional spatial images being taken for different "slices" of the parton momentum fraction, $x$. In different terms, one maps out in this way the $(2+1)$-dimensional structure of the nucleon, with two dimensions in space and one in momentum.

Such spatial images of partons can provide insight into the fundamental questions about QCD dynamics inside hadrons spelled out in sect. 2.1. In particular, quantifying the difference in the distributions of quarks and gluons will shed light on their dynamical interplay, and the dependence of the transverse distribution of quarks on $x$ will reveal to what extent sea and valence quarks have different or similar characteristics. As the size of effects that can be expected is not huge, measurements with high precision are crucial to uncover them.

We will show that with a suitable setup of detectors and the interaction region, the EIC will be able to probe partons at transverse distances $b_{T}$ up to about $1.5 \mathrm{fm}$ or even higher. In this region, there are definite predictions $[98,99]$ for the impact parameter distribution $f\left(x, \boldsymbol{b}_{T}\right)$ of partons, namely an exponential falloff in $b_{T}$ (akin to the one produced by a Yukawa potential) with a characteristic length that depends on $x$ and is of order $1 /\left(2 m_{\pi}\right) \approx 0.7 \mathrm{fm}$. This behavior results from quantum fluctuations with virtual pions at large $b_{T}$, sometimes referred to as the "pion cloud" of the nucleon. The characteristics of these fluctuations are a direct consequence of the breakdown of chiral symmetry in QCD and can be computed using effective field theory methods. From a different point of view, one may hope that the structure of the proton of distances on the femtometer scale will eventually help us to better understand the mechanism of confinement. 
Although the spatial imaging of partons puts highest demands on experiment, the underlying physical principle is quite simple. In suitable exclusive processes one can measure the difference $\boldsymbol{\Delta}_{T}$ between the transverse momentum of the proton in the initial and the final state. A two-dimensional Fourier transform converts the distribution of $\boldsymbol{\Delta}_{T}$ into the spatial distribution of partons in the transverse plane $[100,101]$. This bears some similarity with X-ray diffraction, where a spatial image of a crystal is obtained by a Fourier transform from the deflection of $\mathrm{X}$-rays.

To reconstruct the longitudinal-momentum information in nucleon tomography is less easy. In exclusive processes suitable for parton imaging, the longitudinal momentum of the parton before and after the scattering is in fact not the same. The generalized parton distributions that describe the nucleon structure in these processes thus depend on two momentum fractions, $x+\xi$ and $x-\xi$ as shown in Sidebar IV. Whereas $\xi$ can be directly measured via the longitudinal momentum transferred to the proton, $x$ is integrated over in the expression of the scattering amplitude. However, one finds that the typical values of $x$ in this integral are of order $\xi$. In the first instance, exclusive measurements thus yield integrals over GPDs that can be turned into the distribution of partons with a transverse position $\boldsymbol{b}_{T}$ in the proton and with momentum fractions smeared around $\xi$.

Information about the separate dependence on $x$ and $\xi$ is contained in the dependence of GPDs on the resolution scale $Q^{2}$, given that a change in resolution scale changes their $x$-dependence in a calculable way while leaving $\xi$ and $\boldsymbol{\Delta}_{T}$ untouched. To reconstruct the $x$-dependence of GPDs by measuring the $Q^{2}$-dependence of exclusive processes at given $\xi$ is challenging because the relevant variation in $Q^{2}$ is only logarithmic. To be successful, such a program requires precise data in as wide a range of $Q^{2}$ and $\xi$ as possible.

\section{Orbital motion and angular momentum}

Exclusive processes with polarized beams open up unique possibilities to study spin-orbit correlations of quarks and gluons in the nucleon. A correlation of particular interest is the shift in the transverse distribution of partons induced by transverse polarization $\boldsymbol{S}_{T}$ of the proton, which has the form [100]

$$
f^{\Uparrow}\left(x, \boldsymbol{b}_{T}\right)=f\left(x, \boldsymbol{b}_{T}^{2}\right)+\frac{\left(\boldsymbol{S}_{T} \times \boldsymbol{b}_{T}\right)^{z}}{M} \frac{\partial}{\partial \boldsymbol{b}_{T}^{2}} e\left(x, \boldsymbol{b}_{T}^{2}\right),
$$

where $M$ is the proton mass. The distributions $f\left(x, \boldsymbol{b}_{T}^{2}\right)$ and $e\left(x, \boldsymbol{b}_{T}^{2}\right)$, which give the impact parameter distribution of unpolarized partons and its polarization-induced shift, are respectively obtained by a two-dimensional Fourier transform from the generalized parton distributions $H(x, \xi, t)$ and $E(x, \xi, t)$ at $\xi=0$ (see Sidebar IV). This shift is the position space analog of the Sivers effect discussed in sect. 2.3, where transverse proton polarization induces an anisotropy in the transverse momentum of a parton. The shifts in transverse position and in transverse momentum give independent information about spin-orbit correlations at the parton level.

A dynamical connection between the two phenomena, called chromodynamic lensing, has been formulated in [102]. As explained in sect. 2.3, the Sivers effect arises from the interaction of the scattered parton with the proton remnant. The shift in the spatial distribution of the parton described by eq. (15) goes along with a shift in the spatial distribution of the remnant, which leads to an anisotropy in the transverse momentum of the scattered parton. This connection is explicitly seen in simple model calculations where the proton is represented as a bound state of a quark and a di-quark, with their interaction via gluon exchange being treated in perturbation theory $[95,103]$. At the EIC, it will be possible to measure both the Sivers effect and the GPDs $H$ and $E$ that enter in eq. (15). The comparison of their size, sign and $x$-dependence will yield information about the non-perturbative interactions between active and spectator partons in the nucleon.

The spin-orbit correlation described by eq. (15) is intimately connected with the orbital angular momentum carried by partons in the nucleon and thus with the proton spin puzzle, i.e., with the question of how the spin of the proton is distributed at the microscopic level. Writing the densities in eq. (15) or the associated GPDs in terms of nucleon wave functions, one indeed finds that $E$ originates from the interference of wave functions whose orbital angular momentum differs by one unit [104]. A different way to quantify this connection is Ji's sum rule $[20,105]$

$$
J^{q}=\frac{1}{2} \int \mathrm{d} x x\left[H^{q}(x, \xi, t=0)+E^{q}(x, \xi, t=0)\right],
$$

which represents the total angular momentum $J^{q}$ (including both helicity and orbital contributions) carried by quarks and anti-quarks of flavor $q$ as an integral over GPDs. An analogous relation holds for gluons. There is a close connection between Ji's sum rule and the shift in $b$-space (see eq. (15)) [106]. Let us mention that the very definition of angular momentum for quarks and gluons is non-trivial and involves several conceptual aspects at the core of non-Abelian gauge theories, see e.g. $[21,22]$ and references therein.

$J^{q}$ is a generalized form factor at $t=0$ that can be computed in lattice QCD [6], and we foresee that such computations will have reached maturity by the time the EIC is operational. In turn, a precise determination of eq. (16) with GPDs extracted from exclusive scattering processes is extremely challenging, especially because it requires knowledge of $H$ and $E$ for all $x$ at fixed $\xi$. A reliable estimate of the associated theoretical uncertainties will only be possible when high-precision data enable us to gain a better understanding of the dependence of GPDs on their different kinematic arguments. On the other hand, exclusive scattering experiments can investigate the dependence of $H$ and $E$ on the longitudinal-momentum fractions in a wide kinematic range. Measurements at the EIC will in particular probe the region of sea quarks, whose contribution to the angular momentum sum rule is sup- 
Table 3. Key measurements for imaging partons in the transverse plane. With an EIC running at lower energies, one can investigate the transition from the valence to the sea quark regime and measure the processes in the lower block, while an EIC with higher energies provides access to a wide region dominated by sea quarks and gluons.

\begin{tabular}{|c|c|c|c|}
\hline Deliverables & Observables & What we learn & Requirements \\
\hline \hline GPDs of & DVCS and $J / \psi, \rho^{0}, \phi$ & transverse spatial distrib. & $\int \mathrm{d} t L \sim 10$ to $100 \mathrm{fb}^{-1} ;$ \\
sea quarks & production cross-section & of sea quarks and gluons; & leading proton detection; \\
and gluons & and polarization & total angular momentum & polarized $e^{-}$and $p$ beams; \\
& asymmetries & wide range of $x$ and $Q^{2} ;$ \\
GPDs of & electro-production of & dependence on & range of beam energies; \\
valence and & $\pi^{+}, K$ and $\rho^{+}, K^{*}$ & quark flavor and & $e^{+}$beam \\
sea quarks & & polarization & valuable for DVCS \\
\hline \hline
\end{tabular}

pressed compared to valence quarks because of the factor $x$ in the integral given in eq. (16). In this sense, computations in lattice QCD and measurements of exclusive reactions are highly complementary.

\subsubsection{Processes and observables}

A large number of exclusive channels can be experimentally investigated at the EIC, and each of them will give specific physics information. An overview of key measurements is given in table 3 .

For most processes, we have formal proofs of factorization $[107,108]$, which provide a solid ground for their interpretation in terms of GPDs (akin to the factorization proofs that enable us to extract conventional parton densities from inclusive processes, see sect. 2.2). For these proofs to apply, the photon virtuality $Q^{2}$ must be large, in particular much larger than the invariant momentum transfer $t$ to the hadron. In terms of imaging, the precision $\sim 1 / Q$ with which partons are resolved is then much finer than the precision $\sim 1 / \sqrt{|t|}$ with which their position in the hadron is determined [101]. This permits a clean separation between the object that is being imaged and the probe used to obtain the image.

Deeply virtual Compton scattering (DVCS) is measured in the reaction $e p \rightarrow e p \gamma$ and plays a privileged role in several respects:

- Its theoretical description is most advanced, with radiative corrections being available up to order $\alpha_{s}^{2}[109-$ 111] and corrections of order $1 / Q$ to the limit of large $Q^{2}$ being well understood their structure [112]. Recently, results have even been obtained for corrections of order $1 / Q^{2}$ due to the finite target mass and to nonzero $t$ [113-115].

- It has a large number of angular and polarization observables that can be calculated using the factorization theorem and thus constrain GPDs [116,117]. With longitudinal electron polarization and both longitudinal and transverse polarization of the proton, one has enough observables to disentangle the distributions $H$ and $E$ discussed above, as well as their counterparts $\tilde{H}$ and $\tilde{E}$ for longitudinally polarized partons.
- Several contributions that are suppressed by $1 / Q$ can be extracted from suitable observables and be calculated in terms of twist-three distributions, which are closely connected to those accessible in semiinclusive processes at high transverse momentum (see sect. 2.3.2).

- Compton scattering interferes with the Bethe-Heitler process, which is calculable in QED. This allows one to extract the complex phase of the Compton scattering amplitude, which in turn gives more detailed information about GPDs.

- Further information about the phase of the Compton amplitude can be extracted if both $e^{-}$and $e^{+}$beams are available (even if the latter are unpolarized). In the absence of a positron beam, some of this information may be obtained by running at different beam energies (using a Rosenbluth-type separation of different contributions to the cross-section).

Closely related to DVCS is time-like Compton scattering, $\gamma p \rightarrow \ell^{+} \ell^{-} p$, i.e. photo-production of a lepton pair with large invariant mass $[110,111,118]$. An advantage of this process is that the analog of the DVCS beam charge asymmetry is an asymmetry in the angular distribution of the produced lepton pair, which can be measured without positron beams.

Compton scattering thus has the potential to yield detailed and precise information about GPDs for different polarizations of the partons and the proton. A limitation it shares with inclusive DIS is that it is sensitive only to the sum of quark and anti-quark distributions in a particular flavor combination and that it involves gluon distributions only via a logarithmic dependence on $Q^{2}$. Exclusive meson production offers substantial help in the separation of different quark and anti-quark flavors and of gluons, which is of special interest as discussed in sect. 2.1. The extraction of the flavor dependence of GPDs will only be possible if GPDs are truly universal. Hints of this universality have been unveiled recently by a common analysis of all DVCS and exclusive meson production data with a common GPD set [119]. The theoretical description of these processes is more involved: it requires knowledge of the relevant meson wave functions, and theoretical progress is still needed to achieve control over radiative corrections $[120,121]$ and over corrections to the large $Q^{2}$ limit [122]. Measuring at 
$Q^{2}$ well above $10 \mathrm{GeV}^{2}$ can substantially decrease the theoretical uncertainties. This holds in particular for parton imaging, given that at lower $Q^{2}$ the measured $t$ dependence receives contributions from the finite meson size as well as from the structure of the proton target. Let us highlight specific features of different production channels.

- $J / \psi$ production provides selective access to unpolarized gluons. In this case, the hard scale of the process is $Q^{2}+M_{J / \psi}^{2}$ rather than $Q^{2}$, so that both photoand electro-production can be used to probe GPDs. Electro-production has smaller rates but reduced theoretical uncertainties. Furthermore, the cross-sections $\sigma_{L}$ and $\sigma_{T}$ for longitudinal and transverse photon polarization, which can be separated experimentally from the angular distribution in the decay $J / \psi \rightarrow \ell^{+} \ell^{-}$, provide two independent observables to validate the theory description.

- The production of the neutral vector mesons $\rho^{0}, \phi, \omega$ involves unpolarized gluons and sea quarks in particular flavor combinations. $\rho^{+}$production provides direct information about the difference of $u$ and $d$ distributions, whereas the production of $K^{*}(829)$ is sensitive to strange quarks in the proton [123].

The factorization theorem allows us to compute the cross-section $\sigma_{L}$ for longitudinal photon polarization and the associated transverse proton spin asymmetry, whereas other observables require a model for effects suppressed by $1 / Q$ [124]. An experimental separation of $\sigma_{L}$ and $\sigma_{T}$ can be performed using the vector meson decay, i.e., a Rosenbluth separation with different beam energies is not required.

- Production of the pseudoscalar mesons $\pi, K, \eta$ and $\eta^{\prime}$ provides information about different flavor combinations for longitudinally polarized quarks and antiquarks, encoded in distributions $\tilde{H}$ and $\tilde{E}$. Again, only $\sigma_{L}$ can be computed from the factorization theorem. To separate $\sigma_{L}$ and $\sigma_{T}$ one has to apply the Rosenbluth method and hence needs data for different beam energies.

The calculations of $1 / Q$ suppressed terms in $[125,126]$ found that $\sigma_{T}$ can be of substantial size due to contributions from GPDs for transversely polarized quarks, which are closely related to the transversity distribution $h_{1}(x)$ introduced in Sidebar III.

- The production of $\pi^{+} \pi^{-}$pairs in the continuum or on the $f_{2}(1270)$ resonance is one of the very few processes sensitive to the difference of quark and antiquark distributions [127], thus providing access to the $x$-dependence of the distributions whose integrals over $x$ give the electromagnetic nucleon form factors.

- The production of two mesons with a large rapidity gap between them is again sensitive to GPDs for transversely polarized quarks [128].

Finally there is the possibility to study the generalized parton distributions in the pion using DVCS or meson production on a virtual pion emitted from the proton beam $[98,99,129]$. The experimental signature is a recoil neutron as well as a recoil $\pi^{+}$in the final state. For a clear theoretical interpretation of such a measurement, the emitted pion must have only a small virtuality, i.e., it must be almost real. As shown in [129], this requires both high energy and high luminosity, which will be available at the EIC for the first time.

\subsubsection{Parton imaging now and in the next decade}

Pioneering measurements for imaging low- $x$ partons have been performed in the last decade at the HERA collider, where the experiments H1 and ZEUS measured DVCS and exclusive vector meson production with up to $28 \mathrm{GeV}$ electrons or positrons scattering on $920 \mathrm{GeV}$ unpolarized protons. Most precise information about the spatial gluon distribution comes from $J / \psi$ photo-production (with the smallest statistical errors among all relevant final states), and DVCS has provided us with first information about sea quarks at momentum fractions $x$ around $10^{-3}$. These measurements provide evidence for differences between the spatial distribution of small- $x$ gluons, small- $x$ quarks and the distribution of valence quarks one can infer from the electromagnetic nucleon form factors. For gluons they also show a weak dependence of the average impact parameter on $x$. With an integrated luminosity of $500 \mathrm{pb}^{-1}$ many of the HERA results on imaging are however limited by statistical errors and leave open many important questions, in particular regarding sea quarks and the dependence of impact parameter distributions on the resolution scale $Q^{2}$.

Possibilities to extend the HERA measurements of $\gamma p \rightarrow J / \psi p$ and $\gamma p \rightarrow \Upsilon p$ to higher energies are offered by ultraperipheral proton-proton or proton-nucleus collisions at the LHC. The quasi-real incident photon is radiated off a beam proton or nucleus in this case, the beam particle being scattered with a very low momentum transfer.

Groundbreaking measurements in the region of moderate-to-large $x$ have been made by fixed-target experiments with $28 \mathrm{GeV}$ electrons and positrons at HERMES and with up to $6 \mathrm{GeV}$ electrons at JLab, proving in particular that angular and polarization asymmetries can be measured in DVCS and interpreted in terms of GPDs. However, most of these measurements are at rather small $Q^{2}$ or have sizeable statistical uncertainties, which puts serious limitations on the precision of extracted GPDs and precludes the use of $Q^{2}$ evolution as a tool.

The precise measurements of electromagnetic nucleon form factors, as well as the calculation of generalized form factors in lattice QCD [6], are already providing valuable information about the spatial distribution of partons in regions of $x$ typically above 0.1 or so. Both research areas are anticipated to make significant progress in the future and will constitute an important complement of imaging through exclusive processes, as discussed in sect. 2.1.

First measurements for imaging partons with $x$ between $10^{-1}$ and $10^{-2}$, i.e., in the transition region between valence and sea quarks, will be possible with the COMPASS experiment at CERN, which will have the benefit of both $\mu^{+}$and $\mu^{-}$beams to measure the charge asymmetry in DVCS. The anticipated integrated luminosity around 


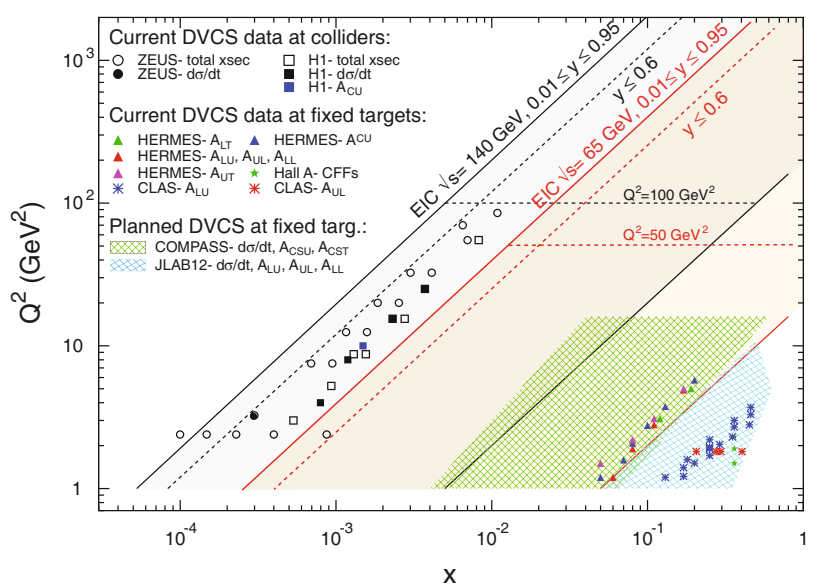

Fig. 24. An overview of existing and planned measurements of DVCS in the $\left(x, Q^{2}\right)$-plane.

$100 \mathrm{pb}^{-1}$ will, however, limit the accuracy of measurements at $Q^{2}$ above $5 \mathrm{GeV}^{2}$ and the possibilities to explore simultaneously the dependence on $x, Q^{2}$ and $t$. At present it is not clear whether polarized protons will be available.

A first era of precise parton imaging will begin with the $12 \mathrm{GeV}$ upgrade at JLab, with very high statistics and sufficiently high $Q^{2}$ to probe partons at high $x$, including the effects of polarization. Figure 24 gives an overview of existing and anticipated measurements of DVCS in the $\left(x, Q^{2}\right)$-plane.

To realize the full physics potential of parton imaging that we have discussed in the previous section will require the EIC. Such a machine will, for the first time, make it possible to image partons with high statistics and with polarization in a wide range of small- to moderate $x$. At high $x$ it will complement the JLab 12 program with measurements at large $Q^{2}$, thus opening up the possibility to extract physics from scaling violations for high-momentum partons.

Let us finally mention that it is very difficult to obtain information on GPDs from exclusive processes in $p+p$ collisions. This is due to the effect of soft gluon exchange between spectator partons in the two protons, which precludes a simple theoretical interpretation of such reactions. Lepton-proton scattering thus provides a privileged way to quantify the spatial structure of the proton via GPDs. On the other hand, the information gained in lepton-proton scattering can help to better understand important features of proton-proton collisions, in particular the dynamics of multi-parton interactions [130,131].

\subsubsection{Accelerator and detector requirements}

The experimental study of DVCS and meson electroproduction requires high luminosity: cross-sections are at best a few percent of the inclusive DIS cross-section, and the data need to be kinematically binned in up to five variables $\left(x, Q^{2}, t, \phi, \phi_{S}\right)$, where $\phi\left(\phi_{S}\right)$ is the angle between the hadron production (proton beam polarization) plane and the electron scattering plane. Luminosities as high as $10^{34} \mathrm{~cm}^{-2} \mathrm{~s}^{-1}$ are crucial for the measurement of DVCS spin asymmetries and for the exploration of the high- $t$ region, as well as for certain meson production channels, especially at low $x$. A large lever arm in $Q^{2}$ at fixed $x$ is required for testing the power behavior predicted by factorization theorems, and beyond this for the use of evolution effects to disentangle gluons from quarks in Compton scattering. If several collision energies and hence several beam configurations are needed to achieve this, one needs accurate measurements of integrated luminosities in order to cross-normalize data sets. A significant lever arm in $y$ at fixed $x$ and $Q^{2}$ is mandatory for the separation of $\sigma_{L}$ and $\sigma_{T}$, which is essential for pseudoscalar mesons and helpful for DVCS in case a positron beam is not available, as explained in sect. 2.4.2.

To measure truly exclusive processes, it is essential to detect all final state particles. Hermeticity of the EIC detector is therefore a crucial requirement. The most critical aspect is the ability to detect the recoil baryon, which in the region of interest has a transverse momentum up to a few $\mathrm{GeV}$. This corresponds to very small scattering angles with respect to the proton beam. At large proton beam energies, the detection of the recoil proton may require Roman Pots integrated in the machine lattice, whereas at lower proton beam energies, or high proton transverse momenta, it should be possible to detect the proton in the main EIC detector. Note that the transverse momentum acceptance is directly related to the region in $b_{T}$ space where reliable images can be obtained. The emittance of the proton beam at the location of the detectors needs to be kept reasonably low so that the detectors can be placed as close to the proton beam as possible. Near perfect hermeticity is also essential in the case of low- $y$ events, which are needed to explore high $x$ at a given $Q^{2}$. Indeed, in this case, $y$ is measured using a hadronic method and depends on the sum over the energy minus the longitudinal momentum of all the hadronic final-state particles.

Specifically for DVCS, but also for $\pi^{0}$ production, the photon detection coverage is particularly important over the full rapidity range. Note that for DVCS, both the photon and the electron tend to be emitted backward in the same hemisphere when the electron energy increases.

As far as particle identification is concerned, the situation varies depending on the beam energies. In the most general case, the separation of electrons and pions requires particular care in the momentum range between about 4 and $10 \mathrm{GeV}$. For the identification of light mesons, mostly in the barrel section, the same care will be necessary in the same momentum range. A ring imaging Cherenkov counter (RICH) or a DIRC complementing a time-of-flight system will likely be needed in the barrel section of the detector (see sect. 6.4). Note that in addition to standard particle identification, the missing-mass method might be used at low collision energies to discriminate between particle types, depending on the kinematics and the resolution that can be achieved. 

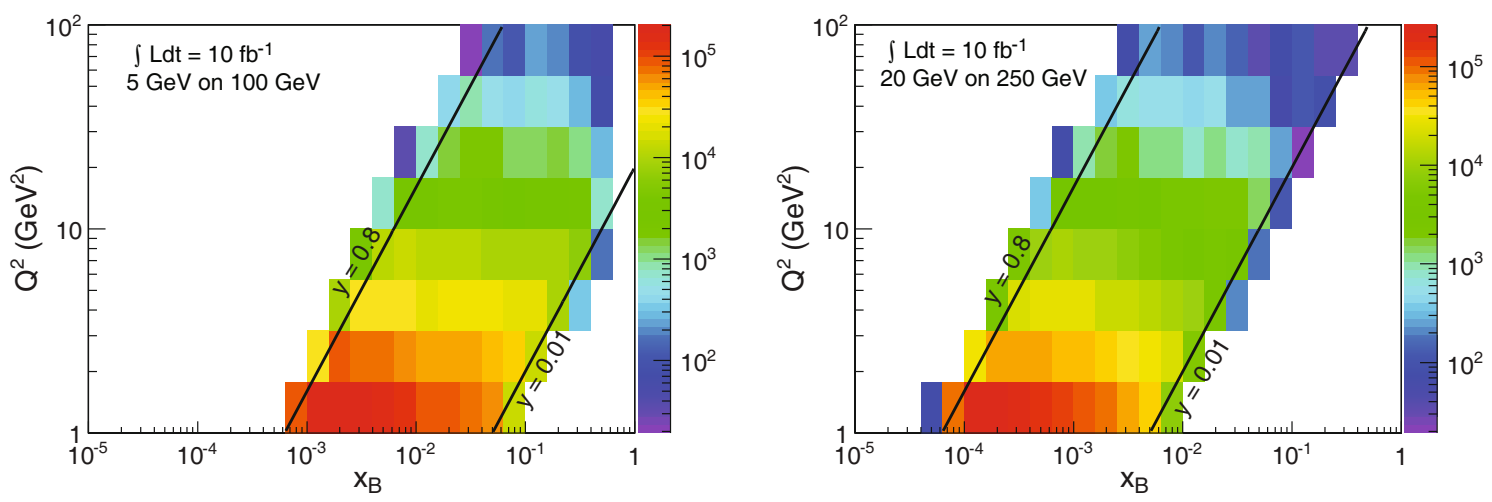

Fig. 25. Expected distribution of DVCS events in bins of $x$ and $Q^{2}$. Event numbers correspond to Compton scattering, i.e. the contribution of the Bethe-Heitler process to the process $e p \rightarrow e p \gamma$ has been subtracted.

To measure $J / \psi$ production, one would use ideally both the decays into $\mu^{+} \mu^{-}$and $e^{+} e^{-}$. In both cases, the momentum resolution needs to be sufficiently good to avoid contamination from the non-resonant background as well as from the exclusive and semi-inclusive $\psi(2 \mathrm{~S})$ production channels, which have the same decay modes.

As pointed out in sect. 2.4.2, polarization is critically important in order to disentangle the different GPDs entering DVCS and other processes. Specifically, transverse proton polarization is essential to access the information about orbital angular momentum encoded in the distribution $E$. High values of electron and especially proton polarization are ideal for precise measurements. The electron and proton polarizations should be measured with sufficient accuracy, so as not to become significant sources of systematic error.

\subsubsection{Parton imaging with the EIC}

Let us show the potential of an EIC for imaging partons using the DVCS process, which plays a privileged role as we discussed in sect. 2.4.2. The following projections are based on events simulated according to GPD models that give a good description of the existing DVCS data $[109,132]$. Acceptance cuts for the detected electron, photon and proton corresponding to the detector layout in sect. 6.2 and sect. 6.4 have been applied. Figure 25 shows that a fine binning of DVCS events in both $x$ and $Q^{2}$ is possible in a wide kinematic range.

With the lower set of beam energies, one finds ample statistics in bins with large $Q^{2}$ for $x$ as high as 0.2 . The combination of such data with fixed-target results will give a substantial lever arm in $Q^{2}$ and permit the study of evolution effects in the kinematic regime where valence quarks are important.

The top panels of fig. 26 show the $t$-dependence of the DVCS cross-section in two bins of $x$ and $Q^{2}$, accessible with $E_{e}=5 \mathrm{GeV}, E_{p}=100 \mathrm{GeV}$ and with $E_{e}=20 \mathrm{GeV}$ $E_{p}=250 \mathrm{GeV}$, respectively. The simulated data have been smeared for resolution, and the error bars include both statistics and an estimate of systematic uncertainties. The scattered proton is assumed to be detected in
Roman pots for $|t|$ above $(175 \mathrm{MeV})^{2}$, see sect. 6.3 and chapt. 7.3 of [2]. More detail on the simulation is given elsewhere [133-135]. From the DVCS cross-section, one can reconstruct the scattering amplitude, which can then be Fourier-transformed into $b_{T}$ space. The resulting images correspond to the particular combination of quarks, anti-quarks and gluons "observed" in Compton scattering. We explained earlier that the momentum fraction of those partons is "smeared" around the measured value of $\xi=x /(2-x)$, whereas the variable $\boldsymbol{b}_{T}$ is legitimately interpreted as a transverse parton position [101]. The bottom panels of fig. 26 show that precise images are obtained in a wide range of $b_{T}$, including the large- $b_{T}$ region where a characteristic dependence on $b_{T}$ and $x$ due to virtual pion fluctuations is predicted as discussed in sect. 2.4.1. We emphasize that a broad acceptance in $t$ is essential to achieve this accuracy. If, for instance, the measured region of $|t|$ starts at $(300 \mathrm{MeV})^{2}$ instead of $(175 \mathrm{MeV})^{2}$, the associated extrapolation uncertainty exceeds $50 \%$ for $b_{T}>1.5 \mathrm{fm}$ with the model used here.

The simulations presented here assume an exponential $t$-dependence of the GPDs and hence of the DVCS cross-section. As shown in sect. 3.6 of [2], GPDs that have a dipole form in $t$ lead to larger uncertainty bands in $b_{T}$ space, with uncertainties becoming significant below $0.2 \mathrm{fm}$. This reflects a larger uncertainty from the extrapolation of the cross-section to the unmeasured large- $t$ region, where a dipole form decreases much less quickly than an exponential law. In such a scenario, measurement up to the largest possible $t$ values is crucial for the accuracy of imaging at small impact parameters.

Figure 27 shows that the quality of EIC measurements allows one to resolve the correlation of the average impact parameter $\left\langle\boldsymbol{b}_{T}^{2}\right\rangle$ with $x$ and with $Q^{2}$. The change of $\left\langle\boldsymbol{b}_{T}^{2}\right\rangle$ with $Q^{2}$ reflects the dynamics of perturbative parton radiation embodied in evolution equations. By contrast, the logarithmic broadening of $\left\langle\boldsymbol{b}_{T}^{2}\right\rangle$ with decreasing $x$ (taken as an input in the GPD model used for the simulation) reflects non-perturbative dynamics, which has been linked to the physics of confinement [136]. To exhibit and separate these effects requires simultaneous binning in $Q^{2}, x$ and $t$ and high precision, which will only be possible at the EIC. 


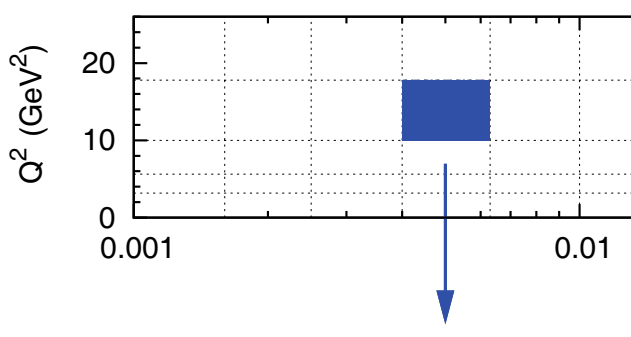

$\gamma^{*}+p \rightarrow \gamma+p$
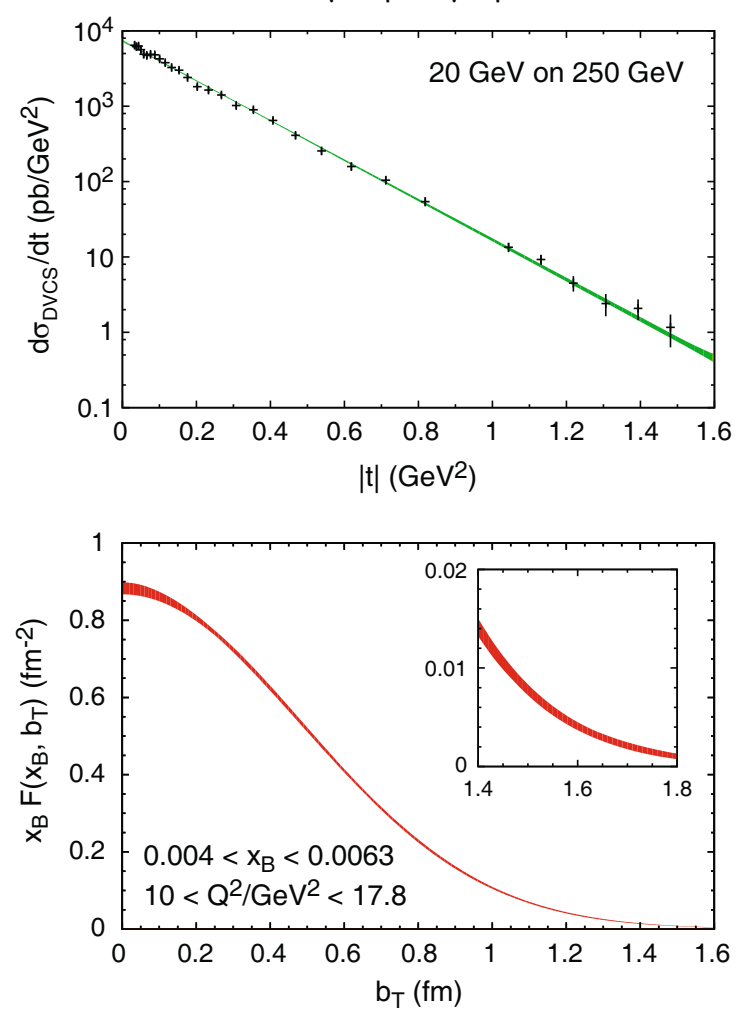

\section{8}
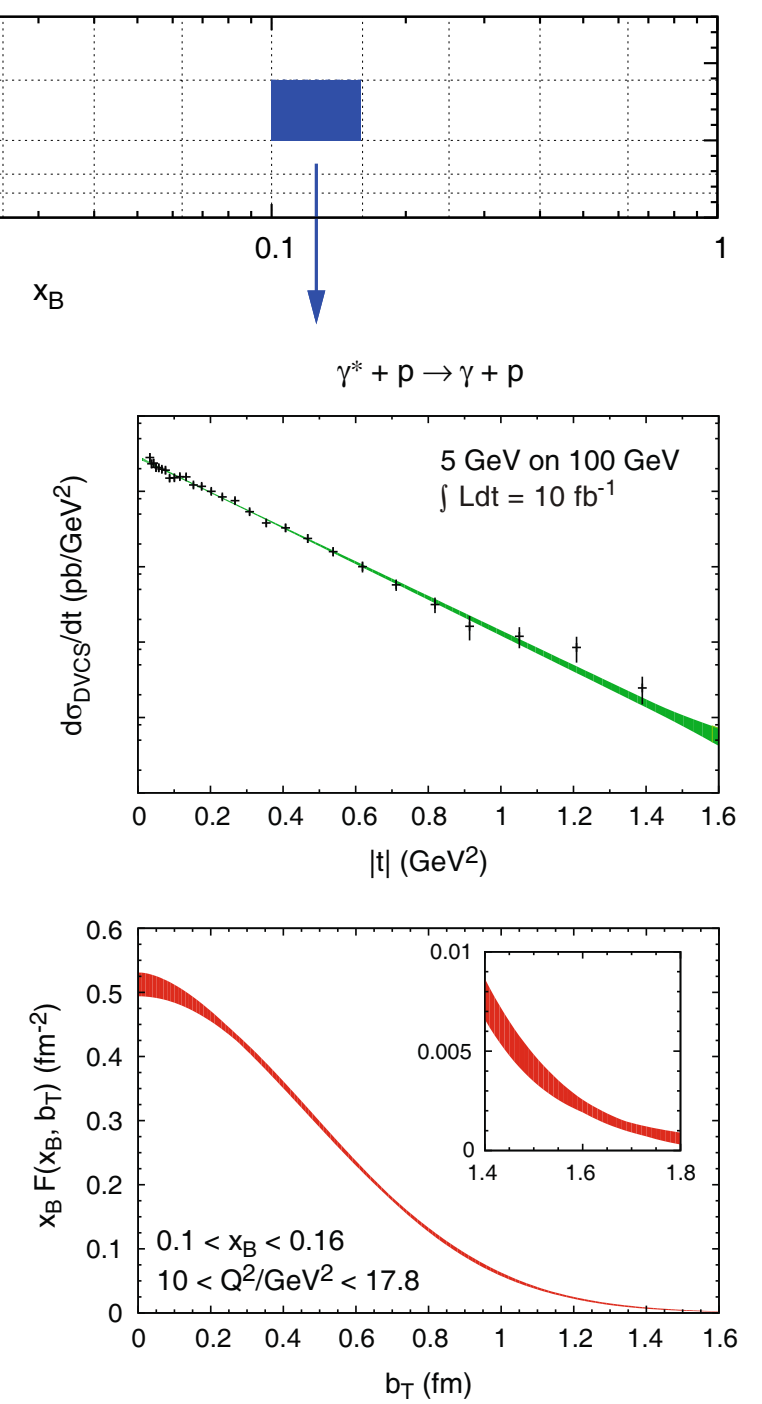

Fig. 26. Top: the DVCS cross-section in two bins of $x$ and $Q^{2}$. The error bars reflect statistical and assumed systematic uncertainties, but not the overall normalization uncertainty from the luminosity measurement. For the left panels the assumed luminosity is $10 \mathrm{fb}^{-1}$ for $|t|<1 \mathrm{GeV}^{2}$ and $100 \mathrm{fb}^{-1}$ for $|t|>1 \mathrm{GeV}^{2}$. Bottom: the distribution of partons in impact parameter $b_{T}$ obtained from the DVCS cross-section. The bands represent the parametric errors in the fit of $\mathrm{d} \sigma_{\mathrm{DVCS}} / \mathrm{d} t$ and the uncertainty from different extrapolations to the regions of unmeasured (very low and very high) $t$, as specified in sect. 3.6 of [2].

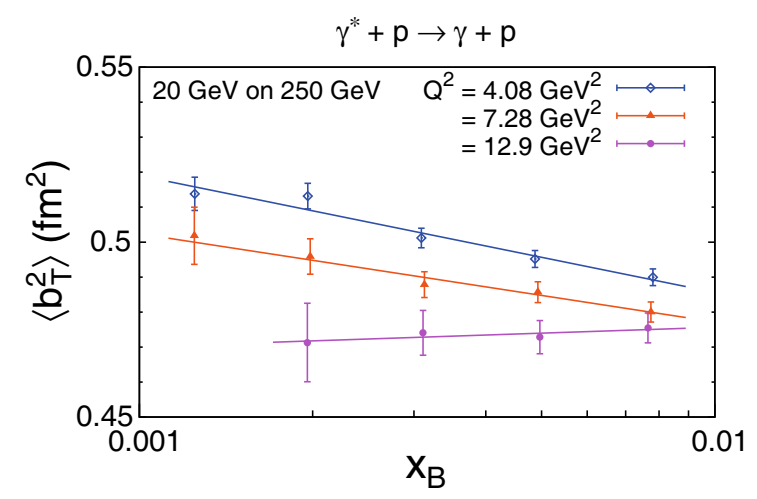

Fig. 27. Average values of $\boldsymbol{b}_{T}^{2}$ obtained from the DVCS crosssection in different bins of $x$ and $Q^{2}$. The assumed luminosity is as for the left panels of fig. 26. The lines indicate linear fits of $\left\langle\boldsymbol{b}_{T}^{2}\right\rangle$ vs. $\log x$ at fixed $Q^{2}$. Within errors, the fit for $Q^{2}=$ $12.9 \mathrm{GeV}^{2}$ is consistent with a vanishing or a small negative slope.
The unpolarized DVCS cross-section is mainly sensitive to the distribution $H$, i.e. to unpolarized partons in an unpolarized proton. Information about the phase of the corresponding amplitude can be extracted from the longitudinal spin asymmetry of the electron beam (not shown here). Sizeable values of this asymmetry are expected for $y$ not too small and not too large (say between 0.2 and 0.8 ). This method can in particular give good constraints in regions where $\mathrm{d} \sigma_{\text {DVCS }} / \mathrm{d} t$ has large uncertainties due to the subtraction of the Bethe-Heitler cross-section.

Information about the other distributions, $E, \tilde{H}$ and $\tilde{E}$, can be extracted from a number of polarization asymmetries. For the sake of simplicity, we focus in the following on the region of small $x$, where $\tilde{H}$ and $\tilde{E}$ are expected to be small and can be neglected in a first approximation. Access to $E$, and thus to orbital angular momentum, can then be obtained from a particular angular asymmetry measurable with transverse proton polarization. The top panel of fig. 28 shows simulated data 

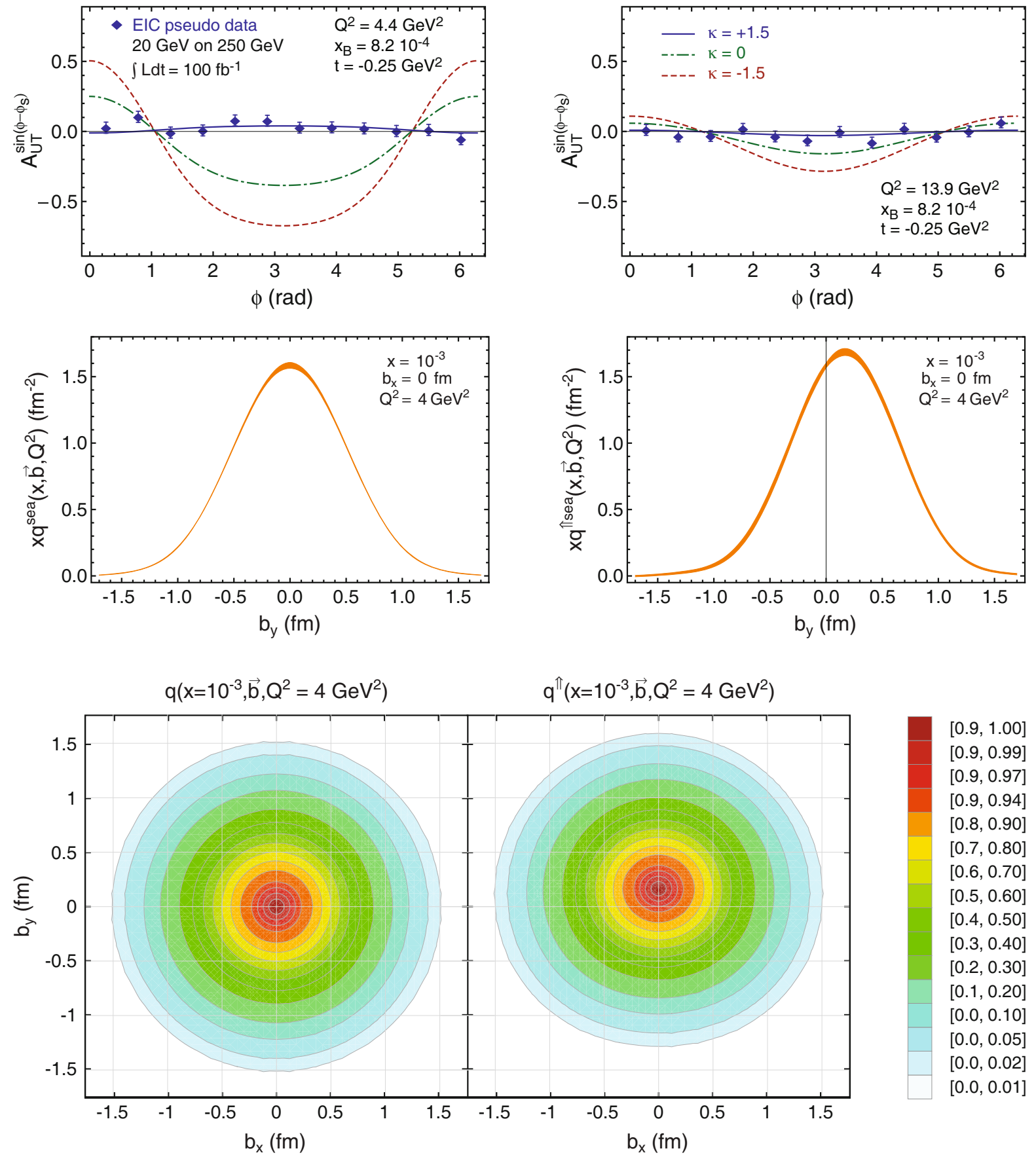

Fig. 28. Top: the DVCS polarization asymmetry $A_{U T}^{\sin \left(\phi-\phi_{S}\right)}$ for a transversely polarized proton (see [138] for a precise definition). Middle: the spatial distribution of sea quarks in an unpolarized proton (left) and in a proton polarized along the positive $x$-axis (right) obtained from a GPD fit to simulated data for $\mathrm{d} \sigma_{\mathrm{DVCS}} / \mathrm{d} t$ and $A_{U T}^{\sin \left(\phi-\phi_{S}\right)}$. The bands represent the parametric errors of the fit and the uncertainty from extrapolating the $t$ spectrum outside the measured region. Bottom: the corresponding density of partons in the transverse plane.

for this asymmetry calculated with a specific model of $E$ and $H$. The curves have been obtained for different values of $\kappa=E(x, \xi, 0) / H(x, \xi, 0)$, which determines the size of the transverse shift in the density (see eq. (15)), and the data points correspond to $\kappa=+1.5$ for sea quarks.
Since the asymmetry receives contributions from both $H$ and $E$ it would be nonzero even for vanishing $E$. The projected errors are for a polarization of $80 \%$ and include estimated systematic uncertainties. We see that the EIC could clearly distinguish between different scenarios. 


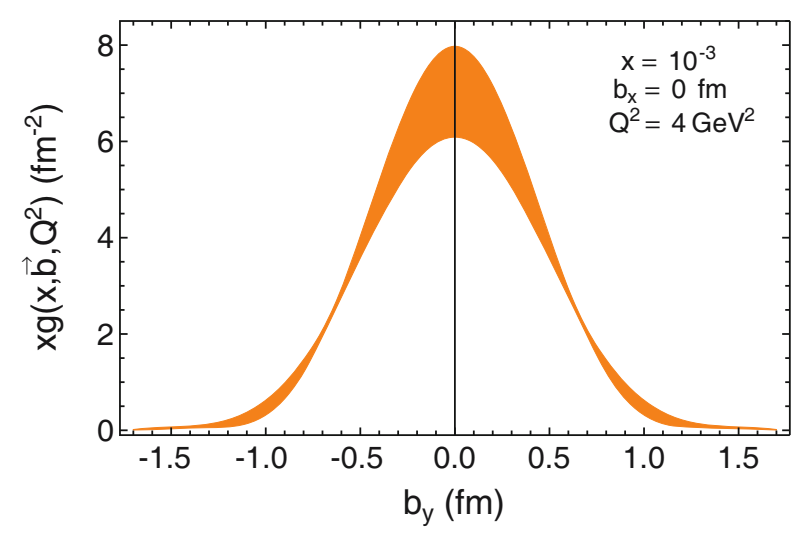

Fig. 29. The $b_{T}$ space density for gluons obtained in the same fit as the densities in fig. 28.

Assuming a functional form of the GPDs, one can extract both $H$ and $E$ in a fit to the DVCS cross-section and the transverse proton spin asymmetry. The middle and lower panels of fig. 28 show the $b_{T}$ space densities obtained from a fit to simulated data for $20 \mathrm{GeV}$ electrons scattering on $250 \mathrm{GeV}$ protons in the kinematic region with $3.2 \mathrm{GeV}^{2}<Q^{2}<17.8 \mathrm{GeV}^{2}$ and $10^{-4}<x<10^{-2}$. Details of this study are given in $[133,137]$. We see that the parametric uncertainty of the results is very small and allows one to resolve the transverse shift of the distribution in a polarized proton (about $0.15 \mathrm{fm}$ in the example). Given its lever arm in $Q^{2}$, the fit also permits a determination of the distribution $H$ for gluons from evolution effects, with the resulting density profile shown in fig. 29.

As discussed in sect. 2.4.2, exclusive $J / \psi$ production offers direct access to the distribution of unpolarized gluons. The scaling variable for this process is $x_{V}$ and the hard scale is $Q^{2}+M_{J / \psi}^{2}$ (see Sidebar IV). The expected distribution of events in $x_{\mathrm{V}}$ and $Q^{2}$ in fig. 30 shows that high-statistics studies will be possible not only for photobut also for electro-production, with the additional benefits mentioned earlier.

Examples for the expected spectrum in $t$ are shown in fig. 31, with details given in [133]. Also shown are the $b_{T}$ space images obtained from the $\gamma^{*} p \rightarrow J / \psi p$ scattering amplitude by a Fourier transform. The distributions thus contain a contribution from the small but finite size of the $J / \psi$ meson, which needs to be disentangled in a full GPD analysis. We see from the figure that with data from the low- and high-energy coverage of an EIC, this process will enable us to accurately probe the spatial distribution of gluons over two orders of magnitude in $x$, up to the region where the dominant partons are valence quarks. The transverse proton spin asymmetry [139] will in addition give constraints on the distribution $E$ for gluons and thus strongly complement what can be achieved with DVCS.

\subsubsection{Opportunities with nuclei}

Although the focus of this section is on imaging the proton, let us briefly point out that exclusive reactions with nuclear beams offer a variety of physics opportunities. Light nuclei such as ${ }^{3} \mathrm{He}$ or the deuteron can provide an ef- fective neutron target, which can be used for disentangling $u$ and $d$ distributions, just as for the usual parton densities measured in inclusive processes. Such measurements are even more powerful if the nuclei can be polarized.

Coherent exclusive processes, in which the nucleus stays intact, give new handles for the understanding of collective dynamics such as shadowing, anti-shadowing or the EMC effect. An overview and references can be found in sect. 5.9.1 of [2]. Coherent exclusive reactions such as $J / \psi$ production on heavy nuclear targets have the potential to map out the geometry of the nucleus in high-energy processes and thus to quantify the initial conditions of heavy-ion collisions. As discussed in sect. 3.2.2, they may offer detailed information about parton saturation by exhibiting the $\boldsymbol{b}_{T}$ dependence of the amplitude $N\left(x, \boldsymbol{r}_{T}, \boldsymbol{b}_{T}\right)$ for scattering a color dipole of size $\boldsymbol{r}_{T}$ at a transverse distance $\boldsymbol{b}_{T}$ from the center of the nucleus.

Scattering processes at high $Q^{2}$ in which two or more nucleons are simultaneously knocked out of a nucleus provide an opportunity to study short-range correlations between nucleons in a nucleus. Fixed-target experiments $[140,141]$ have obtained intriguing results, which not only provide detailed insight into the nucleon-nucleon interaction at short distances but also have astrophysical implications [142]. At the EIC, one will have the unique opportunity to study the role of gluon degrees of freedom in these short-range correlations. For instance, in exclusive $J / \psi$ production off light nuclei accompanied by knockout nucleons, see sect. 5.12 of [2]. Such studies have the potential to greatly increase our understanding of nuclear forces in the transition region between hadronic and partonic degrees of freedom.

\section{The nucleus: a laboratory for QCD}

\subsection{Introduction}

QCD, the accepted theory of strong interactions, is in general very successful in describing a broad range of hadronic and nuclear phenomena. One of the main achievements in our understanding of QCD is the variation of the strong coupling constant and asymptotic freedom, which is the name for the theoretically predicted and experimentally established fact that quarks and gluons are almost free at very short (asymptotic) distances inside the hadrons $[143,144]$. QCD is often studied in deep inelastic scattering (DIS) experiments, in which one probes the inner structure of the proton or nucleus by scattering a small probe (a lepton) on it. The lepton probes the quark distribution in the proton or nucleus by exchanging a photon with it. Past DIS experiments were very successful in determining the quark structure of the proton and of some light and intermediate-size nuclei.

Despite the many successes in our understanding of QCD, some profound mysteries remain. One of them is quark confinement: quarks can not be free (for a long time) in nature and are always confined inside bound states - the hadrons. Another one is the mass of the proton (and other hadrons), which, at $938 \mathrm{MeV}$, is much larger than the sum of the valence quark masses (about $10 \mathrm{MeV}$ ). 

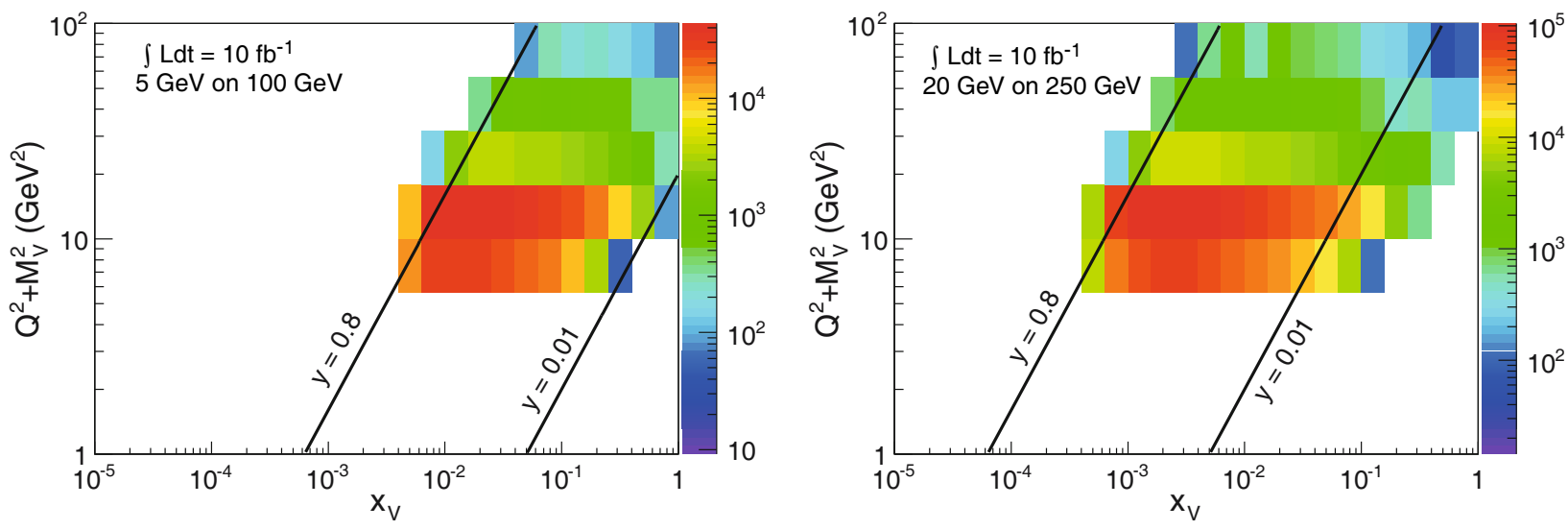

Fig. 30. Expected number of events for exclusive $J / \psi$ production in bins of $x_{V}$ and $Q^{2}$.
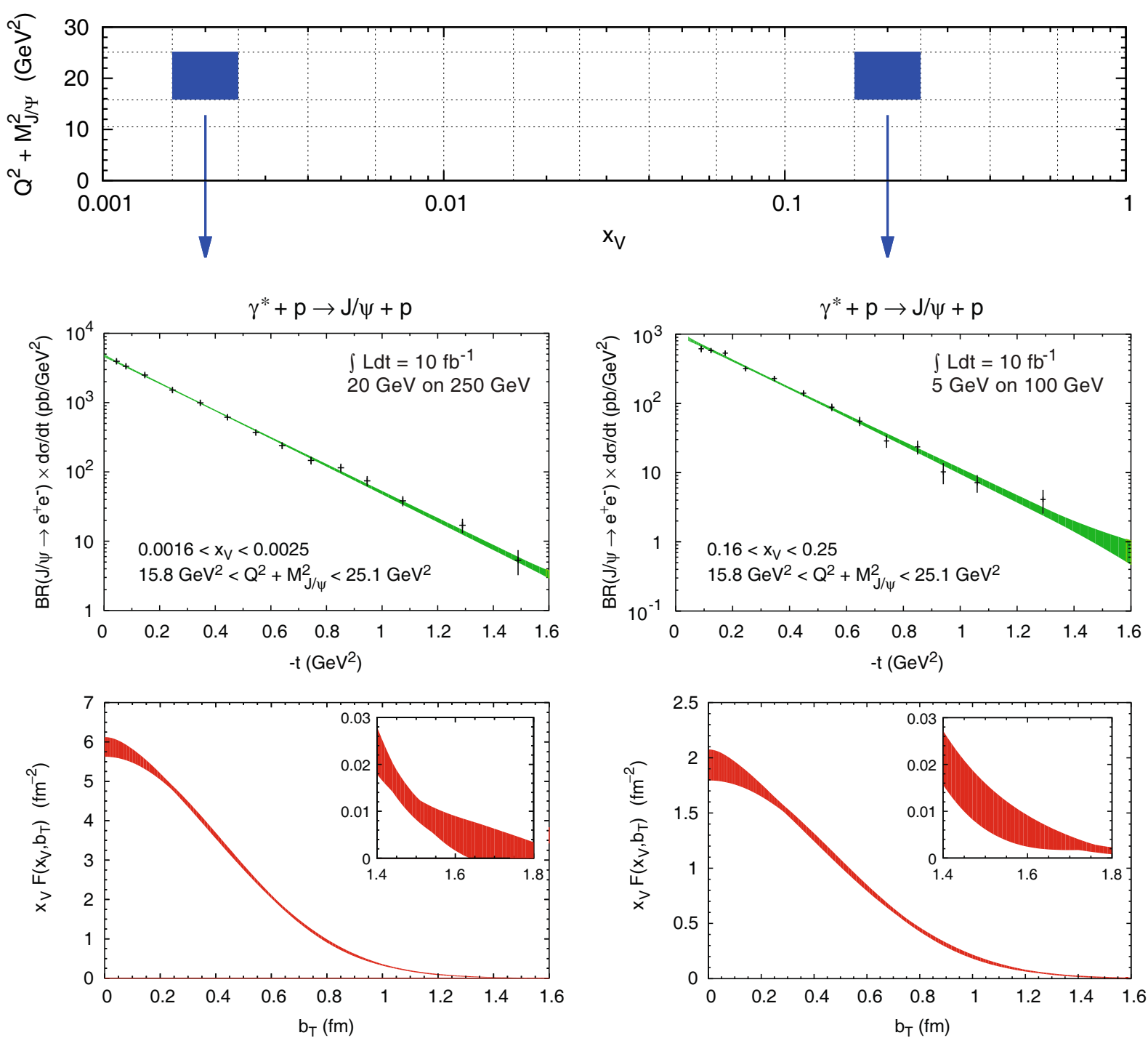

Fig. 31. Top: cross-section for $\gamma^{*} p \rightarrow J / \psi p$ in two bins of $x_{V}$ and $Q^{2}$. Bottom: the distribution of gluons in impact parameter $b_{T}$ obtained from the $J / \psi$ production cross-section. The bands have the same meaning as in fig. 26 . 
Both of these problems at the moment can only be tackled by numerical QCD simulations on the lattice. The current consensus is that the gluons are responsible for both the quark confinement and much of the hadronic mass. The gluons, which bind quarks together into mesons (bound states of a quark and an anti-quark) and baryons (bound states of three quarks), significantly contribute to the masses of hadrons. At the same time, gluons are significantly less well-understood than quarks. Unlike photons, the carriers of the electromagnetic force, gluons interact with each other. The underlying non-linear dynamics of this self-interaction is hard to put under theoretical control. Gluons are quite little-studied for particles providing over $98 \%$ of the proton and neutron masses, generating much of the visible matter mass in the Universe ${ }^{5}$. In addition, it is known that gluons play a dominant role in high-energy DIS, hadronic and nuclear collisions, being responsible for much of the particle production and total cross-sections in these processes. In high-energy heavy-ion collisions it is the gluons that are likely to be responsible for production and thermalization of the medium made out of deconfined quarks and gluons, known as the quarkgluon plasma (QGP). Clearly any progress in our understanding of gluon dynamics would profoundly improve our knowledge of the strong force, allowing us to better control and more deeply understand this fundamental interaction.

In this section, we illustrate that DIS experiments on large nuclei (heavy ions) at high energies are the best way to study gluon dynamics. We show that a large number of nucleons in a heavy ion likely results in strong gluon fields in its wave function probed at high energy, possibly leading to the phenomenon of parton (gluon) saturation, also known as the Color Glass Condensate (CGC). The transition to this non-linear regime is characterized by the saturation momentum $Q_{s}$, which can be large for heavy ions. Our current theoretical understanding suggests that this strong gluon field combines complex non-linear QCD dynamics with a perturbatively large momentum scale $Q_{s}$, allowing one to perform small-coupling theoretical calculations due to the asymptotic freedom property of QCD. An electron-ion collider (EIC) would allow us to probe the wave functions of high-energy nuclei with an energetic electron: by studying these interactions one may probe the strong gluon fields of the CGC. While experiments at HERA, RHIC, and LHC found evidence consistent with saturation, an EIC would have the potential to seal the case, completing the discovery process started at those accelerators.

Nuclei are made out of nucleons, which in turn, are bound states of the fundamental constituents probed in high-energy scattering or at short distance, namely quarks and gluons. The binding of nucleons into a nucleus must be sensitive to how these quarks and gluons are confined into

\footnotetext{
${ }^{5}$ One may compare the gluons to the Higgs boson, the search for which received a lot of attention in recent decades. While the recently discovered Standard Model Higgs accounts for the masses of all the known quarks along with the $W^{ \pm}$and $Z$ bosons, this would still add up to only about $5 \%$ of the mass in the visible universe.
}

nucleons, and must influence how they distribute inside the bound nucleons. The European Muon Collaboration (EMC) discovery at CERN that revealed a peculiar pattern of nuclear modification of the DIS cross-section as a function of Bjorken $x$, confirmed by measurements at several facilities in the following two decades, shows clear evidence that the momentum distributions of quarks in a fast-moving nucleus are strongly affected by the binding and the nuclear environment. With much wider kinematic reach in both $x$ and $Q$, and unprecedented high luminosity, the EIC not only can explore the influence of the binding on the momentum distribution of sea quarks and gluons, but also, for the first time, determine the spatial distribution of quarks and gluons in a nucleus by diffractive or exclusive processes.

The EIC is capable of exploring the emergence of hadrons from almost massless quarks and gluons, or heavy quarks. This is a necessary and critical process in the formation of our visible universe shortly after its birth. Color neutralization is key to the formation of hadrons, and is still not understood within QCD. In electron-nucleus $(e+\mathrm{A})$ collisions at the EIC, the nucleus could serve as an effective femtometer size detector to probe the color neutralization of a fast moving color charge. With the span of available collision energies, the wealth of semi-inclusive probes and the control of kinematics, the EIC is able to explore the response of nuclear medium to the motion of the color charge, and to probe the strength and spatial distributions of quarks and gluons inside the colliding nucleus.

The EIC would be the world's first dedicated electronnucleus $(e+\mathrm{A})$ collider. It would be an excellent laboratory for exploring QCD dynamics. The experimental program of the machine is targeted to answer the following fundamental questions concerning the dynamics of quarks and gluons in a nuclear environment:

- Can we experimentally find evidence of a novel universal regime of non-linear QCD dynamics in nuclei? The large number of partons in a nucleus may result in strong gluon fields leading to the phenomenon of gluon saturation, known as the Color Glass Condensate. This universal regime of high-energy QCD is described by non-linear evolution equations. Discovery of the saturation regime would not be complete without unambiguous experimental evidence in favor of this non-linear behavior that stands in strong contrast to the linear DGLAP evolution, which describes QCD at large- $x$ and $Q^{2}$ so successfully. An EIC can complete the discovery of the gluon saturation/CGC regime, tantalizing hints of which may have been seen at HERA, RHIC, and the LHC. Accomplishing the discovery of a new regime of QCD would have a profound impact on our understanding of strong interactions.

- What is the role of saturated strong gluon fields, and what are the degrees of freedom in this high gluon density regime? An EIC will allow us to probe the wave functions of high-energy nuclei. By studying these interactions, one may probe the strong gluon fields of the CGC, possibly the strongest fields in nature. In 
this regime, multi-parton correlations dominate and the picture of hadronic matter described by individual parton distributions loses its validity. If quarks and gluons are not the relevant degrees of freedom any more, than what are the correct degrees of freedom? With its broad kinematic range, an EIC will allow us to explore this small- $x$ regime and gain insight into the dynamic of saturation expanding our understanding of QCD.

- What is the fundamental quark-gluon structure of light and heavy nuclei? The measurement of momentum and spatial (impact parameter) distributions of gluons and sea quarks in nuclei over an unprecedented kinematic range in $x$ and $Q^{2}$ would provide groundbreaking insight into the new regime of saturation and the fundamental structure of nuclei. These measured distributions at the EIC, together with the understanding of quark and gluon correlations, could expand our knowledge of nuclear structure into the realm of fundamental interaction described by QCD.

- Can the nucleus, serving as a color filter, provide novel insight into the propagation, attenuation and hadronization of colored quarks and gluons? The emergence of colorless hadrons from colored quarks and gluons is a rich and still mysterious process in QCD. Multiple interactions between a moving color charge and the color field of a nucleus it is colliding with, could alter the color evolution of this charge and its hadronization. Hence, it is a valuable probe of color neutralization. By using the nucleus as a space-time analyzer the EIC will shed light on answers to the questions such as the following: How does the nucleus respond to the propagation of a color charge through it? What are the fluctuations in the spatial distributions of quarks and gluons inside the nucleus? What governs the transition from quarks and gluons to hadrons?

The big questions listed above can be answered by performing a set of measurements using DIS on heavy ions at the EIC. The measurements relevant for the small- $x e+\mathrm{A}$ physics are described in sect. 3.2, while those pertaining to the large- $x e+\mathrm{A}$ physics are discussed in sect. 3.3. Some of these measurements have analogs in $e+p$ collisions but have never been performed in nuclei; for these, $e+p$ collisions will allow us to understand universal features of the physics of the nucleon and the physics of nuclei. Other measurements have no analog in $e+p$ collisions and nuclei provide a completely unique environment to explore these. The EIC would have a capability of colliding many ion species at a wide range of collision energies. With its high luminosity and detector coverage, as well as its high collision energies, the EIC could probe the confined motion as well as spatial distributions of quarks and gluons inside a nucleus at unprecedented resolution - one tenth of a femtometer or better - and could detect soft gluons whose energy in the rest frame of the nucleus is less than one tenth of the averaged binding energy needed to hold the nucleons together to form the nucleus. With large nuclei, the EIC could reach the saturation regime that may only be reached by electron-proton collisions with a multi-

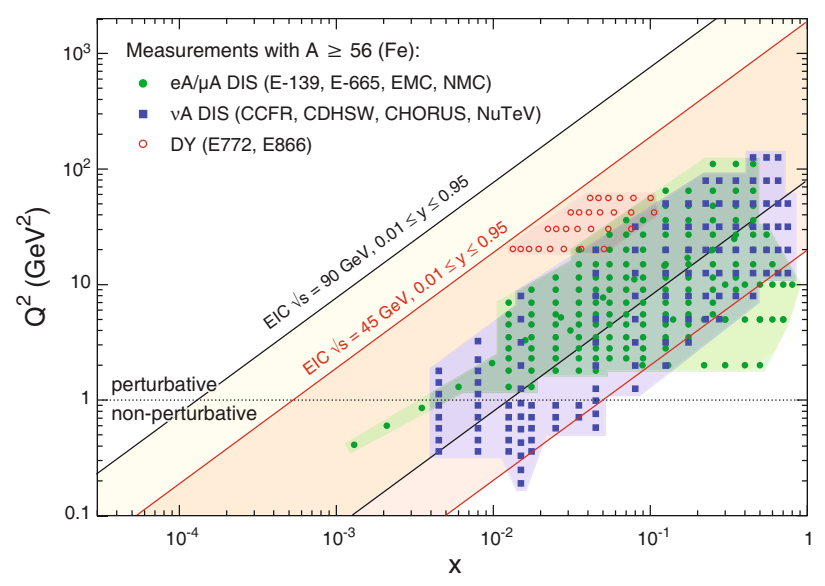

Fig. 32. The kinematic acceptance in $x$ and $Q^{2}$ of completed lepton-nucleus (DIS) and Drell-Yan (DY) experiments (all fixed target) compared to two EIC energy options. The acceptance bands for the EIC are defined by $Q^{2}=x y s$ with $0.01 \leq y \leq 0.95$ and values of $s$ shown.

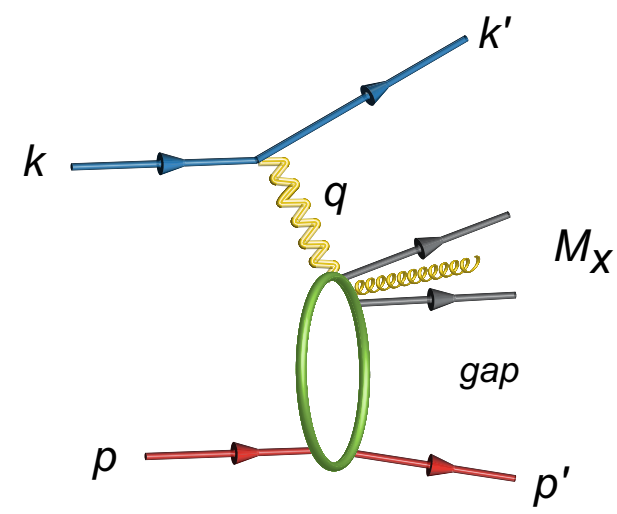

Fig. 33. Kinematic quantities for the description of a diffractive event.

$\mathrm{TeV}$ proton beam. The kinematic acceptance of an EIC compared to all other data collected in DIS on nuclei and in Drell-Yan (DY) experiments is shown in fig. 32. Clearly an EIC would greatly extend our knowledge of strong interactions in a nuclear environment.

\section{Sidebar V. Diffractive scattering}

Diffractive scattering has made a spectacular comeback with the observation of an unexpectedly large crosssection for diffractive events at the HERA $e+p$ collider. At HERA, hard diffractive events, $e(k)+N(p) \rightarrow$ $e^{\prime}\left(k^{\prime}\right)+N\left(p^{\prime}\right)+X$, were observed where the proton remained intact and the highly virtual photon fragmented into a final state $X$ that was separated from the scattered proton by a large rapidity gap without any particles. These events are indicative of a color neutral exchange in the $t$ channel between the virtual photon and the proton over several units in rapidity. This color singlet exchange has historically been called the pomeron, which had a specific interpretation in Regge theory. An illustration of a hard diffractive event is shown in fig. 33 . 
The kinematic variables are similar to those for DIS with the following additions:

$t=\left(p-p^{\prime}\right)^{2}$ is the square of the momentum transfer at the hadronic vertex. The variable $t$ here is identical to the one used in exclusive processes and generalised parton distributions (see Sidebar IV).

$M_{X}^{2}=\left(p-p^{\prime}+k-k^{\prime}\right)^{2}$ is the squared mass of the diffractive final state.

$\eta=\ln (\tan (\theta / 2))$ is the pseudorapidity of a particle whose momentum has a relative angle $\theta$ to the proton beam axis. For ultra-relativistic particles the pseudorapidity is equal to the rapidity, $\eta \sim y=1 / 2 \ln \left(\left(E+p_{L}\right) /(E-\right.$ $\left.\left.p_{L}\right)\right)$.

At HERA, gaps of several units in rapidity have been observed. One finds that roughly $15 \%$ of the deep inelastic cross-section corresponds to hard diffractive events with invariant masses $M_{X}>3 \mathrm{GeV}$. The remarkable nature of this result is transparent in the proton rest frame: a $50 \mathrm{TeV}$ electron slams into the proton and $\approx 15 \%$ of the time, the proton is unaffected, even though the virtual photon imparts a high momentum transfer on a quark or anti-quark in the target. A crucial question in diffraction is the nature of the color neutral exchange between the proton and the virtual photon. This interaction probes, in a novel fashion, the nature of confining interactions within hadrons.

The cross-section can be formulated analogously to inclusive DIS by defining the diffractive structure functions $F_{2}^{D}$ and $F_{L}^{D}$ as

$$
\begin{aligned}
& \frac{\mathrm{d}^{4} \sigma}{\mathrm{d} x_{B} \mathrm{~d} Q^{2} \mathrm{~d} M_{X}^{2} \mathrm{~d} t}= \\
& \frac{4 \pi \alpha^{2}}{Q^{6}}\left[\left(1-y+\frac{y^{2}}{2}\right) F_{2}^{D, 4}\left(x, Q^{2}, M_{X}^{2}, t\right)\right. \\
& \left.-\frac{y^{2}}{2} F_{L}^{D, 4}\left(x, Q^{2}, M_{X}^{2}, t\right)\right] .
\end{aligned}
$$

In practice, detector specifics may limit the measurements of diffractive events to those where the outgoing proton (nucleus) is not tagged, requiring instead a large rapidity gap $\Delta \eta$ in the detector. $t$ can then only be measured for particular final states $X$, e.g. for $J / \Psi$ mesons, whose momentum can be reconstructed very precisely.

\subsection{Physics of high gluon densities in nuclei ${ }^{6}$}

In this section we present a description of the physics one would like to access with the small- $x$ EIC program, along with the measurements needed to answer the related fundamental questions from the beginning of this section. One needs to measure the nuclear structure functions $F_{2}$ and $F_{L}$ (see Sidebar II) as functions of the Bjorken- $x$ variable and photon virtuality $Q^{2}$ (see Sidebar I), which allows us to extract quark and gluon distribution functions of the nuclei, along with the experimental evidence for the

${ }^{6}$ Conveners: Yuri Kovchegov and Thomas Ullrich. non-linear QCD effects. One needs to determine the saturation scale $Q_{s}$ characterizing the CGC wave function by measuring two-particle correlations. The distribution of gluons, both in position and momentum spaces, can be pinpointed by the measurement of the cross-section of elastic vector meson production. The cross-sections for diffractive (quasi-elastic) events are most sensitive to the onset of the non-linear QCD dynamics.

\subsubsection{Gluon saturation: a new regime of $Q C D$}

\section{Non-linear evolution}

The proton is a bound state of three "valence" quarks: two up quarks and one down quark. The simplest view of a proton reveals three quarks interacting via the exchanges of gluons, which "glue" the quarks together. But experiments probing proton structure at the HERA collider at Germany's DESY laboratory, and the increasing body of evidence from RHIC and the LHC, suggest that this picture is far too simple. Countless other gluons and a "sea" of quarks and anti-quarks pop in and out of existence within each hadron. These fluctuations can be probed in high-energy scattering experiments. Due to Lorentz time dilation, the more we accelerate a proton and the closer it gets to the speed of light, the longer are the lifetimes of the gluons that arise from the quantum fluctuations. An outside "observer" viewing a fast moving proton would see the cascading of gluons last longer and longer, the larger the velocity of the proton. So, in effect, by speeding the proton up, one can slow down the gluon fluctuations enough to "take snapshots" of them with a probe particle sent to interact with the high-energy proton.

In DIS experiments, one probes the proton wavefunction with a lepton, which interacts with the proton by exchanging a (virtual) photon with it (see Sidebar I). The virtuality of the photon, $Q^{2}$, determines the size of the region in the plane transverse to the beam axis probed by the photon. By the uncertainty principle, the region's width is $\Delta r_{T} \sim 1 / Q$. Another relevant variable is Bjorken $x$, which is the fraction of the proton momentum carried by the struck quark. At high energy, $x \approx Q^{2} / W^{2}$ is small $\left(W^{2}\right.$ is the center-of-mass energy squared of the photonproton system). Therefore, small $x$ corresponds to highenergy scattering.

The proton wave function depends on both $x$ and $Q^{2}$. An example of such a dependence is shown in fig. 34, extracted from the data measured at HERA for DIS on a proton. Here we plot the $x$-dependence of the parton (quark or gluon) distribution functions (PDFs). At the leading order PDFs can be interpreted as providing the number of quarks and gluons with a certain fraction $x$ of the proton's momentum. In fig. 34, one can see the PDFs of the valence quarks in the proton, $x u_{v}$ and $x d_{v}$ which decrease with decreasing $x$. The PDFs of the "sea" quarks and gluons, denoted by $x G$ and $x S$ in fig. 34, appear to grow very strongly towards the low $x$. (Please note the logarithmic scale of the vertical axis.) One can also observe that the gluon distribution dominates over those of the 


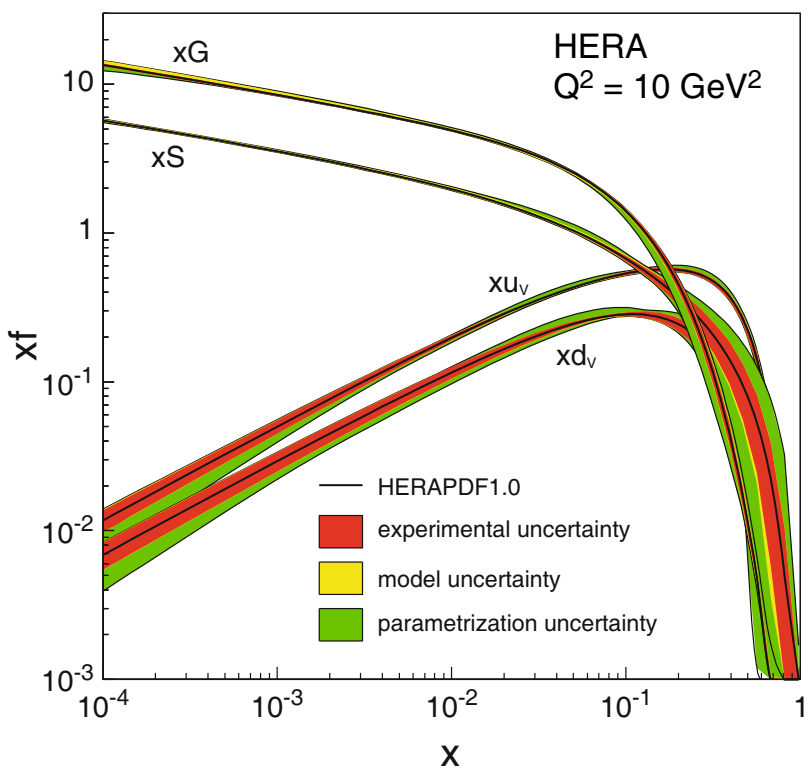

Fig. 34. Proton parton distribution functions plotted as functions of Bjorken $x$. Clearly gluons dominate at small- $x$.

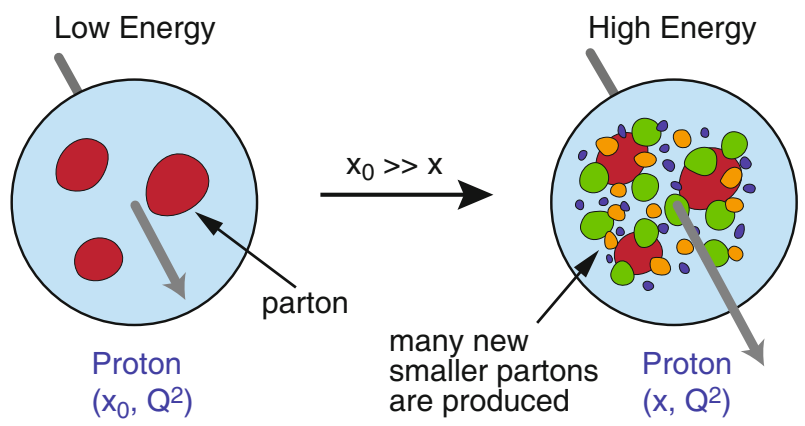

"Color Glass Condensate"

Fig. 35. The proton wave function at small $x$ (shown on the right) contains a large number of gluons (and quarks) as compared to the same wave function at a larger $x=x_{0}$ (shown on the left). The figure is a projection on the plane transverse to the beam axis (the latter is shown by arrows coming "out of the page," with the length of the arrows reflecting the momentum of the proton).

valence and "sea" quarks at a moderate $x$ below $x=0.1$. Remembering that low $x$ means high energy, we conclude that the part of the proton wave function responsible for the interactions in high energy scattering consists mainly of gluons.

The small- $x$ proton wave function is dominated by gluons, which are likely to populate the transverse area of the proton, creating a high density of gluons. This is shown in fig. 35, which illustrates how at lower $x$ (right panel), the partons (mainly gluons) are much more numerous inside the proton than at larger $x$ (left panel), in agreement with fig. 34 . This dense small- $x$ wave function of an ultrarelativistic proton or nucleus is referred to as the Color Glass Condensate (CGC) [145].
To understand the onset of the dense regime, one usually employs $Q C D$ evolution equations. The main principle is as follows: While the current state of the QCD theory does not allow for a first-principles calculation of the quark and gluon distributions, the evolution equations, looselyspeaking, allow one to determine these distributions at some values of $\left(x, Q^{2}\right)$ if they are initially known at some other $\left(x_{0}, Q_{0}^{2}\right)$. The most widely used evolution equation is the Dokshitzer-Gribov-Lipatov-Altarelli-Parisi (DGLAP) equation [12-14]. If the PDFs are specified at some initial virtuality $Q_{0}^{2}$, the DGLAP equation allows one to find the parton distributions at $Q^{2}>Q_{0}^{2}$ at all $x$ where DGLAP evolution is applicable. The evolution equation that allows one to construct the parton distributions at low $x$, given the value of it at some $x_{0}>x$ and all $Q^{2}$, is the Balitsky-Fadin-Kuraev-Lipatov (BFKL) evolution equation $[146,147]$. This is a linear evolution equation, which is illustrated by the first term on the right-hand side of fig. 36. The wave function of a high-energy proton or nucleus containing many small- $x$ partons is shown on the left of fig. 36. As we make one step of evolution by boosting the nucleus/proton to higher energy in order to probe its smaller- $x$ wave function, either one of the partons can split into two partons, leading to an increase in the number of partons proportional to the number of partons $N$ at the previous step,

$$
\frac{\partial N\left(x, r_{T}\right)}{\partial \ln (1 / x)}=\alpha_{s} K_{\mathrm{BFKL}} \otimes N\left(x, r_{T}\right),
$$

with $K_{\mathrm{BFKL}}$ an integral kernel and $\alpha_{s}$ the strong coupling constant. In DIS at high energy, the virtual photon splits into a quark-antiquark dipole which interacts with the proton. The dipole scattering amplitude $N\left(x, r_{T}\right)$ probes the gluon distribution in the proton at the transverse distance $r_{T} \sim 1 / Q$ (see footnote $\left.{ }^{7}\right)$. Note that a Fourier transform of $N\left(x, r_{T}\right)$ is related to the gluon transverse momentum distribution (TMD) $f\left(x, k_{T}\right)$ from sect. 2 . The BFKL evolution leads to the power-law growth of the parton distributions with decreasing $x$, such that $N \sim(1 / x)^{\lambda}$ with $\lambda$ a positive number [146]. This behavior may account for the increase of the gluon density at small $x$ in the HERA data of fig. 34 .

The question arises whether the gluon and quark densities can grow without limit at small $x$. While there is no strict bound on the number density of gluons in QCD, there is a bound on the scattering cross-sections stemming from unitarity. Indeed, a proton (or nucleus) with a lot of "sea" gluons is more likely to interact in high energy scattering, which leads to larger scattering cross-sections. Therefore, the bound on cross-sections should have implications for the gluon density. The cross-section bound arises due to the black disk limit known from quantum mechanics. The high-energy total scattering cross section of a particle on a sphere of radius $R$ is bounded by

$$
\sigma_{\text {tot }} \leq 2 \pi R^{2}
$$

${ }^{7}$ In general, the dipole amplitude also depends on the impact parameter $b_{T}$ of the dipole (cf. sect. 2.4.6): for simplicity we suppress this dependence in $N\left(x, r_{T}\right)$. 


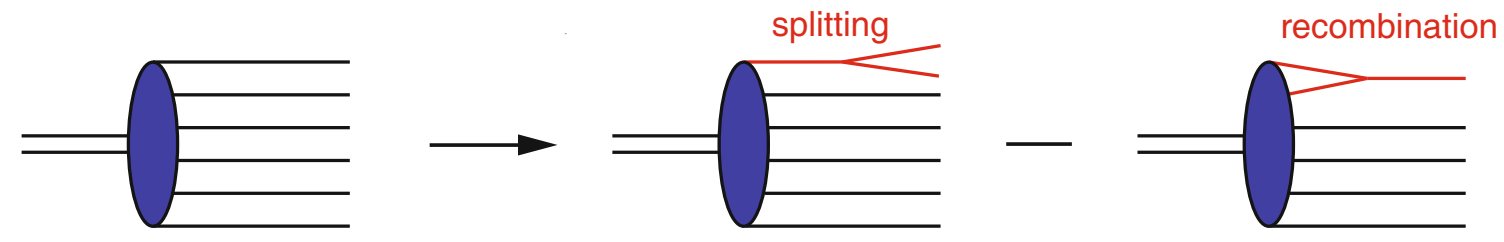

Fig. 36. The non-linear small- $x$ evolution of a hadronic or nuclear wave functions. All partons (quarks and gluons) are denoted by straight solid lines for simplicity.

In QCD, the black disk limit translates into the FroissartMartin unitarity bound, which states that the total hadronic cross-section can not grow faster than $\ln ^{2} s$ at very high energies with $s$ the center-of-mass energy squared [148]. The cross section resulting from the BFKL growth of the gluon density in the proton or nucleus wavefunction grows as a power of energy, $\sigma_{\text {tot }} \sim s^{\lambda}$, and clearly violates both the black disk limit and the Froissart-Martin bound at very high energy.

We see that something has to modify the BFKL evolution at high energy to prevent it from becoming unphysically large. The modification is illustrated on the far right of fig. 36. At very high energies (leading to high gluon densities), partons may start to recombine with each other on top of the splitting. The recombination of two partons into one is proportional to the number of pairs of partons, which in turn scales as $N^{2}$. We end up with the following non-linear evolution equation:

$$
\frac{\partial N\left(x, r_{T}\right)}{\partial \ln (1 / x)}=\alpha_{s} K_{\mathrm{BFKL}} \otimes N\left(x, r_{T}\right)-\alpha_{s}\left[N\left(x, r_{T}\right)\right]^{2} .
$$

This is the Balitsky-Kovchegov (BK) evolution equation [149-151], which is valid for QCD in the limit of the large number of colors $N_{c}$ (see footnote ${ }^{8}$ ). A generalization of eq. (19) beyond the large- $N_{c}$ limit is accomplished by the Jalilian-Marian-Iancu-McLerranWeigert-Leonidov-Kovner (JIMWLK) [145, 154-157] evolution equation, which is a functional differential equation.

The physical impact of the quadratic term on the right of eq. (19) is clear: it slows down the small- $x$ evolution, leading to parton saturation, when the number density of partons stops growing with decreasing $x$. The corresponding total cross-sections satisfy the black disk limit of eq. (18). The effect of gluon mergers becomes important when the quadratic term in eq. (19) becomes comparable to the linear term on the right-hand side. This gives rise to the saturation scale $Q_{s}$, which grows as $Q_{s}^{2} \sim(1 / x)^{\lambda}$ with decreasing $x[152,158,159]$.

\section{Classical gluon fields and the nuclear "Oomph" factor}

\footnotetext{
8 An equation of this type was originally suggested by Gribov, Levin and Ryskin in [152] and by Mueller and Qiu in [153], though at the time it was assumed that the quadratic term was only the first non-linear correction with higher order terms expected to be present as well. In $[149,150]$, the exact form of the equation was found, and it was shown that in the large- $N_{c}$ limit eq. (19) does not have any higher-order terms in $N$.
}

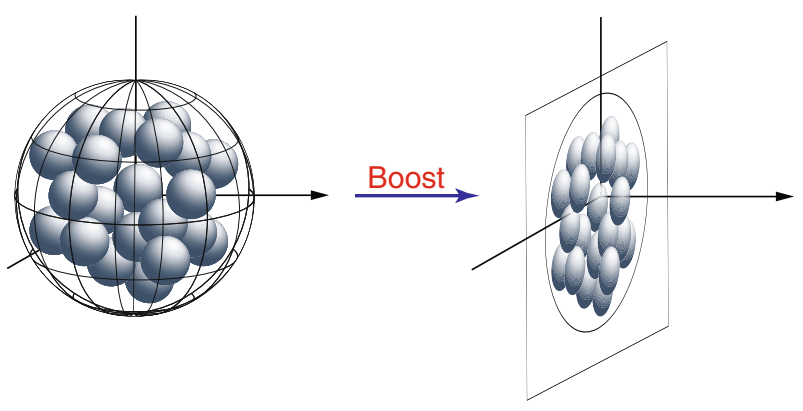

Fig. 37. A large nucleus before and after an ultra-relativistic boost.

We have argued above that parton saturation is a universal phenomenon, valid both for scattering on a proton or a nucleus. Here we demonstrate that nuclei provide an extra enhancement of the saturation phenomenon, making it easier to observe and study experimentally.

Imagine a large nucleus (a heavy ion), which was boosted to some ultra-relativistic velocity, as shown in fig. 37. We are interested in the dynamics of small- $x$ gluons in the wave function of this relativistic nucleus. One can show that due to the Heisenberg uncertainty principle, the small- $x$ gluons interact with the whole nucleus coherently in the longitudinal (beam) direction, Therefore, only the transverse plane distribution of nucleons is important for the small- $x$ wave function. As one can see from fig. 37, after the boost, the nucleons, as "seen" by the small- $x$ gluons with large longitudinal wavelength, appear to overlap with each other in the transverse plane, leading to high parton density. A large occupation number of color charges (partons) leads to a classical gluon field dominating the small- $x$ wave-function of the nucleus. This is the essence of the McLerran-Venugopalan (MV) model [160]. According to the MV model, the dominant gluon field is given by the solution of the classical YangMills equations, which are the QCD analogue of Maxwell equations of electrodynamics.

The Yang-Mills equations were solved for a single nucleus exactly $[161,162]$; their solution was used to construct an unintegrated gluon distribution (gluon TMD) $\phi\left(x, k_{T}^{2}\right)$ shown in fig. 38 (multiplied by the phase space factor of the gluon's transverse momentum $k_{T}$ ) as a function of $k_{T}$ (see footnote ${ }^{9}$ ). Figure 38 demonstrates the emergence of the saturation scale $Q_{s}$. The majority of

\footnotetext{
${ }^{9}$ Note that in the MV model $\phi\left(x, k_{T}^{2}\right)$ is independent of Bjorken $x$. Its $x$-dependence comes in through the BK/ JIMWLK evolution equations described above.
} 


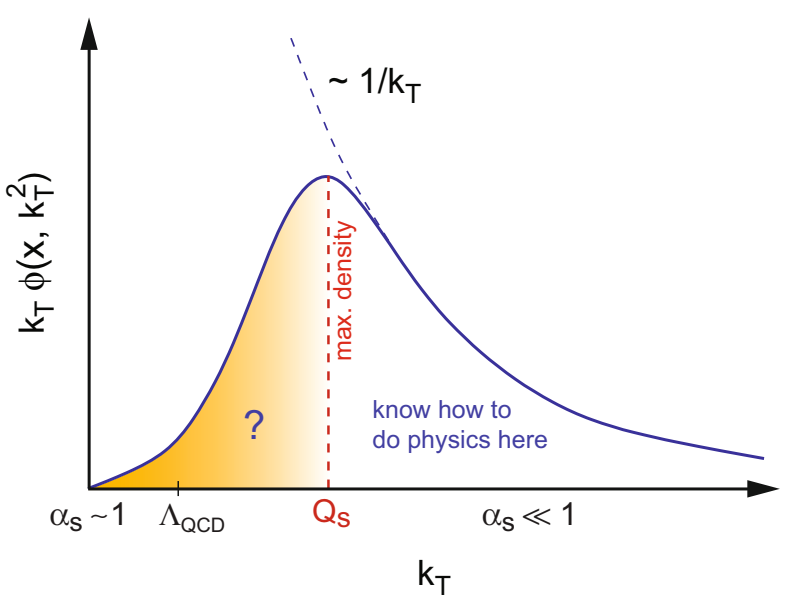

Fig. 38. The unintegrated gluon distribution (gluon TMD) $\phi\left(x, k_{T}^{2}\right)$ of a large nucleus due to classical gluon fields (solid line). The dashed curve denotes the lowest-order perturbative result.

gluons in this classical distribution have transverse momentum $k_{T} \approx Q_{s}$. Note that the gluon distribution slows down its growth with decreasing $k_{T}$ for $k_{T}<Q_{s}$ (from a power-law of $k_{T}$ to a logarithm, as can be shown by explicit calculations). The distribution saturates, justifying the name of the saturation scale.

The gluon field arises from all the nucleons in the nucleus at a given location in the transverse plane (impact parameter). Away from the edges, the nucleon density in the nucleus is approximately constant. Therefore, the number of nucleons at a fixed impact parameter is simply proportional to the thickness of the nucleus in the longitudinal (beam) direction.

For a large nucleus, that thickness, in turn, is proportional to the nuclear radius $R \sim A^{1 / 3}$ with the nuclear mass number $A$. The transverse momentum of the gluon can be thought of as arising from many transverse momentum "kicks" acquired from interactions with the partons in all the nucleons at a given impact parameter. Neglecting the correlations between nucleons, which is justified for a large nucleus in the leading power of $A$ approximation, once can think of the "kicks" as being random. Just like in the random walk problem, after $A^{1 / 3}$ random kicks the typical transverse momentum - and hence the saturation scale- becomes $Q_{s} \sim \sqrt{A^{1 / 3}}$, such that $Q_{s}^{2}, \sim A^{1 / 3}$. We see that the saturation scale for heavy ions, $Q_{s}^{A}$ is much larger than the saturation scale of the proton, $Q_{s}^{p}$, (at the same $x$ ), since $\left(Q_{s}^{A}\right)^{2} \approx A^{1 / 3}\left(Q_{s}^{p}\right)^{2}[152,153,160,163]$. This enhancement factor $A^{1 / 3}$ of the saturation scale squared is often referred to as the nuclear "oomph" factor, since it reflects the enhancement of saturation effects in the nucleus as compared to the proton. For the gold nucleus with $A=197$, the nuclear "oomph" factor is $A^{1 / 3} \approx 6$.

\section{Map of high energy QCD and the saturation scale}

We summarize our theoretical knowledge of high energy QCD discussed above in fig. 39, in which different regimes are plotted in the $\left(Q^{2}, Y=\ln 1 / x\right)$ plane. On

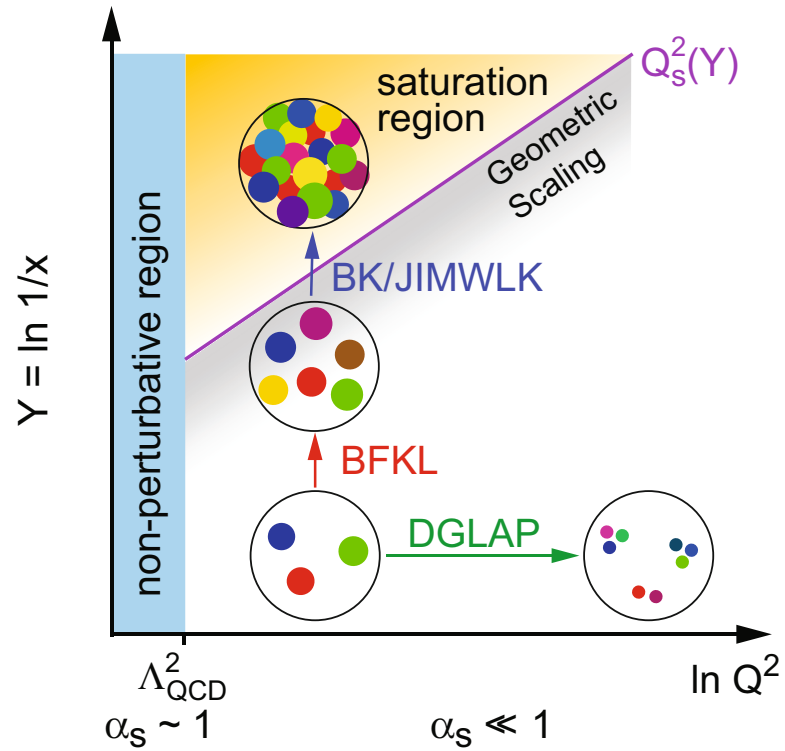

Fig. 39. The map of high energy QCD in the $\left(Q^{2}, Y=\ln 1 / x\right)$ plane.

the left of fig. 39 we see the region with $Q^{2} \leq \Lambda_{Q C D}^{2}$ in which the strong coupling is large, $\alpha_{s} \sim 1$, and smallcoupling approaches do not work $\left(\Lambda_{Q C D}\right.$ is the QCD confinement scale). In the perturbative region, $Q^{2} \gg \Lambda_{Q C D}^{2}$, where the coupling is small, $\alpha_{s} \ll 1$, we see the standard DGLAP evolution and the linear small- $x$ BFKL evolution, denoted by the horizontal and vertical arrows correspondingly. The BFKL equation evolves the gluon distribution towards small- $x$, where the parton density becomes large and parton saturation sets in. The transition to saturation is described by the non-linear BK and JIMWLK evolution equations. Most importantly, this transition happens at $Q_{s}^{2} \gg \Lambda_{Q C D}^{2}$ where the small-coupling approach is valid.

Saturation/CGC physics provides a new way of tackling the problem of calculating hadronic and nuclear scattering cross-sections. It is based on the theoretical observation that small- $x$ hadronic and nuclear wave-functions - and, therefore, the scattering cross-sections - are described by an internal momentum scale, the saturation scale $Q_{s}$ [152]. As we argued above, the saturation scale grows with decreasing $x$ (and, conversely, with the increasing center-of-mass energy $\sqrt{s}$ ) and with the increasing mass number of a nucleus $A$ (in the case of a nuclear wave function) approximately as

$$
Q_{s}^{2}(x) \sim A^{1 / 3}\left(\frac{1}{x}\right)^{\lambda}
$$

where the best current theoretical estimates of $\lambda$ give $\lambda=0.2-0.3$ [164], in agreement with the experimental data collected at HERA [165-168] and at RHIC [164]. Therefore, for hadronic collisions at high energy and/or for collisions of large ultra-relativistic nuclei, the saturation scale becomes large, $Q_{s}^{2} \gg \Lambda_{Q C D}^{2}$. For the total (and particle production) cross-sections, $Q_{s}$ is usually the largest momentum scale in the problem. We therefore expect it 

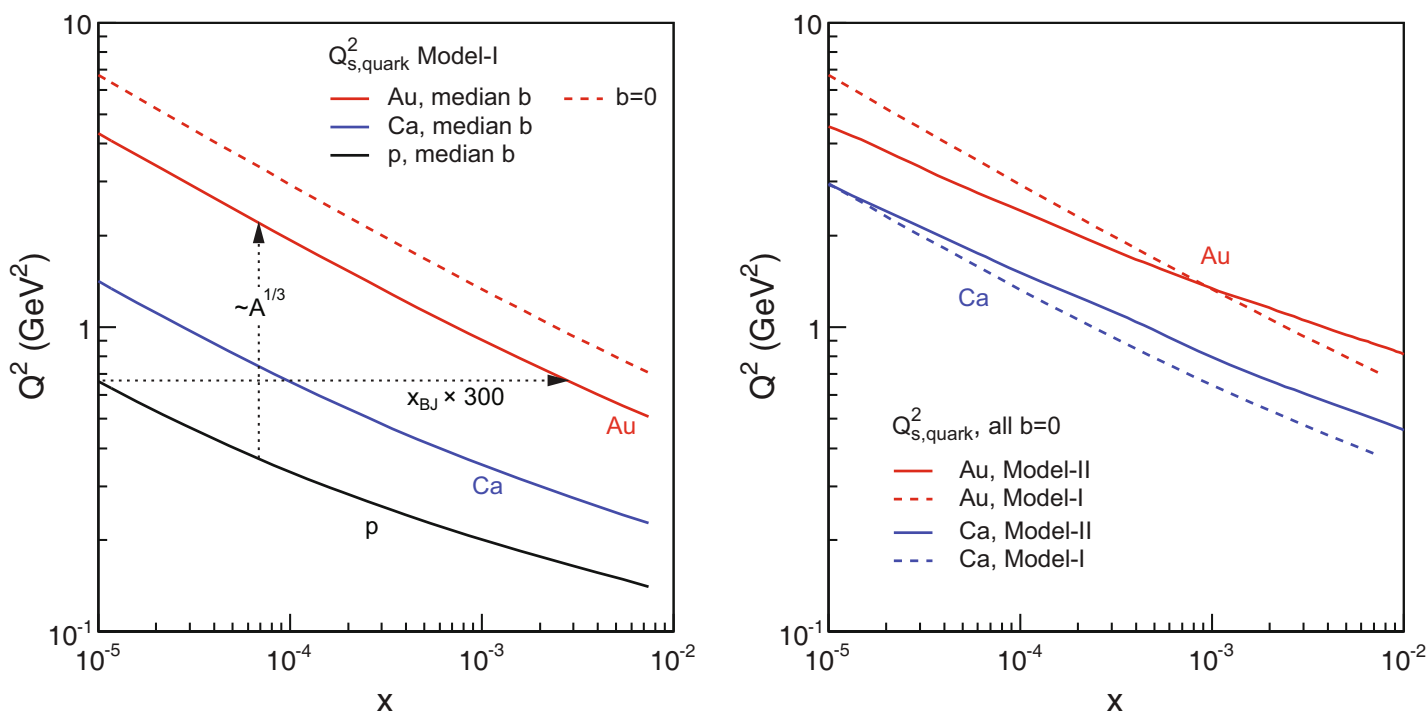

Fig. 40. Theoretical expectations for the saturation scale as a function of Bjorken $x$ for the proton along with Ca and Au nuclei.

to be the scale determining the value of the running QCD coupling constant, making it small,

$$
\alpha_{s}\left(Q_{s}^{2}\right) \ll 1,
$$

and allowing for first-principles calculations of total hadronic and nuclear cross-sections, along with extending our ability to calculate particle production and to describe diffraction in a small-coupling framework. For detailed descriptions of the physics of parton saturation and the CGC, we refer the reader to the review articles [169-172] and to an upcoming book [173].

Equation (20) can be written in the following simple pocket formula if one puts $\lambda=1 / 3$, which is close to the range of $\lambda$ quoted above. One has

$$
Q_{s}^{2}(x) \sim\left(\frac{A}{x}\right)^{1 / 3} .
$$

From the pocket formula (22), we see that the saturation scale of the gold nucleus $(A=197)$ is as large as that for a proton at the 197 times smaller value of $x$ ! Since lower values of $x$ can only be achieved by increasing the centerof-mass energy, which could be prohibitively expensive, we conclude that at the energies available at the modernday colliders one is more likely to complete the discovery of saturation/CGC physics started at HERA, RHIC, and the LHC by performing DIS experiments on nuclei.

This point is further illustrated in fig. 40, which shows our expectations for the saturation scale as a function of $x$ coming from the saturation-inspired Model-I [174] and from the prediction of the BK evolution equation (with higher-order perturbative corrections included in its kernel) dubbed Model-II [165,166]. One can clearly see from the left panel that the saturation scale for $\mathrm{Au}$ is larger than the saturation scale for $\mathrm{Ca}$, which, in turn, is much larger than the saturation scale for the proton: the "oomph" factor of large nuclei is seen to be quite significant.
As we argued above, the saturation scale squared is proportional to the thickness of the nucleus at a given impact parameter $b$. Therefore, the saturation scale depends on the impact parameter, becoming larger for small $b \approx 0$ (for scattering through the center of the nucleus, see fig. 37) and smaller for large $b \approx R$ (for scattering on the nuclear periphery, see fig. 37). This can be seen in the left panel of fig. 40 where most values of $Q_{s}$ are plotted for median $b$ by solid lines, while, for comparison, the $Q_{s}$ of gold is also plotted for $b=0$ by the dashed line: one can see that the saturation scale at $b=0$ is larger than at median $b$. The curves in the right panel of fig. 40 are plotted for $b=0$ : this is why they give higher values of $Q_{s}$ than the median- $b$ curves shown in the left panel for the same nuclei.

This $A$-dependence of the saturation scale, including a realistic impact parameter dependence, is the raison d'être for an electron-ion collider. Collisions with nuclei probe the same universal physics as seen with protons at values of $x$ at least two orders of magnitude lower (or equivalently an order of magnitude larger $\sqrt{s}$ ). Thus, the nucleus is an efficient amplifier of the universal physics of high gluon densities allowing us to study the saturation regime in $e+\mathrm{A}$ at significantly lower energy than would be possible in $e+p$. For example, as can be seen from fig. $40, Q_{s}^{2} \approx$ $7 \mathrm{GeV}^{2}$ is reached at $x=10^{-5}$ in $e+p$ collisions requiring a collider providing a center-of-mass energy of almost $\sqrt{s} \approx$ $\sqrt{Q_{s}^{2} / x} \approx 1 \mathrm{TeV}$, while in $e+\mathrm{Au}$ collisions, only $\sqrt{s} \approx$ $60 \mathrm{GeV}$ is required to achieve comparable gluon density and the same saturation scale.

To illustrate the conclusion that $Q_{s}$ is an increasing function of both $A$ and $1 / x$, we show a plot of its dependence on both variables in fig. 41 using Model-I of fig. 40. One can see again from fig. 41 that larger $Q_{s}$ can be obtained by increasing the energy or by increasing mass number $A$. 


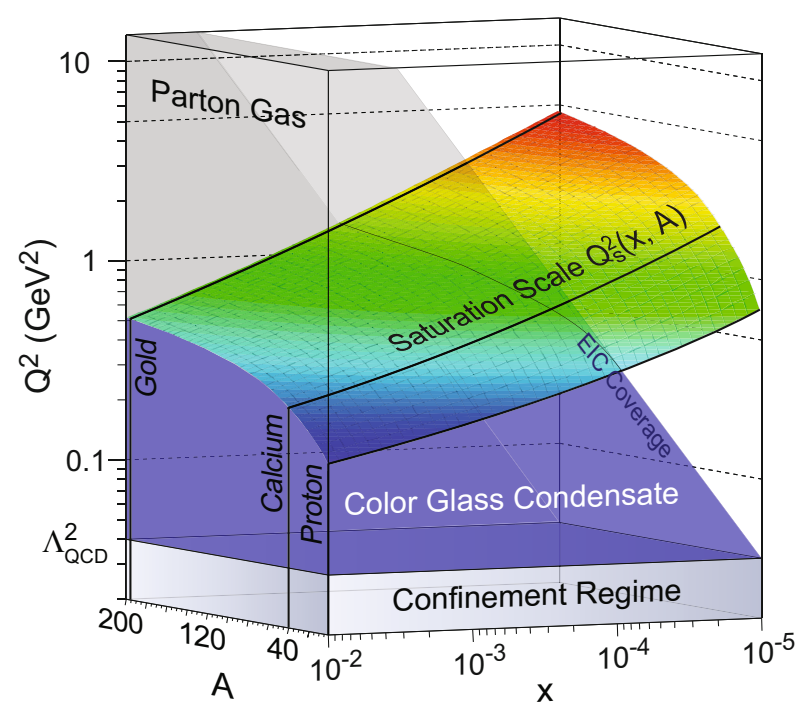

Fig. 41. The theoretical expectations for the saturation scale at medium impact parameter from Model-I as a function of Bjorken $x$ and the nuclear mass number $A$.

Measurements extracting the $x, b$ and $A$ dependence of the saturation scale provide very useful information on the momentum distribution and space-time structure of strong color fields in QCD at high energies. The saturation scale defines the transverse momentum of the majority of gluons in the small- $x$ wave-function, as shown in fig. 38, thus being instrumental to our understanding of the momentum distributions of gluons. The impact parameter dependence of the saturation scale tells us how the gluons are distributed in the transverse coordinate plane, clarifying the spatial distribution of the small- $x$ gluons in the proton or nucleus.

\section{Nuclear structure functions}

The plots in figs. 39, 40 and 41 suggest a straightforward way of finding saturation/CGC physics: if we perform the DIS experiment on a proton, or, better yet, on a nucleus, and measure the DIS scattering cross-section as a function of $x$ and $Q^{2}$, then, at sufficiently low $x$ and $Q^{2}$, one may be able to see the effects of saturation. As explained in Sidebar II, the total DIS cross-section is related to the structure functions $F_{2}\left(x, Q^{2}\right)$ and $F_{L}\left(x, Q^{2}\right)$ by a linear relation. One finds that the structure function $F_{2}$ is more sensitive to the quark distribution $x q\left(x, Q^{2}\right)$ of the proton or nucleus, while the structure function $F_{L}$ measures the gluon distribution $x G\left(x, Q^{2}\right)[12,175]$. Saturation effects can thus be seen in both $F_{2}$ and $F_{L}$ at low $x$ and $Q^{2}$, although, since saturation is gluon-driven, one would expect $F_{L}$ to manifest them stronger.

The nuclear effects on the structure functions can be quantified by the ratios

$$
\begin{aligned}
R_{2}\left(x, Q^{2}\right) & \equiv \frac{F_{2}^{A}\left(x, Q^{2}\right)}{A F_{2}^{p}\left(x, Q^{2}\right)}, \\
R_{L}\left(x, Q^{2}\right) & \equiv \frac{F_{L}^{A}\left(x, Q^{2}\right)}{A F_{L}^{p}\left(x, Q^{2}\right)},
\end{aligned}
$$

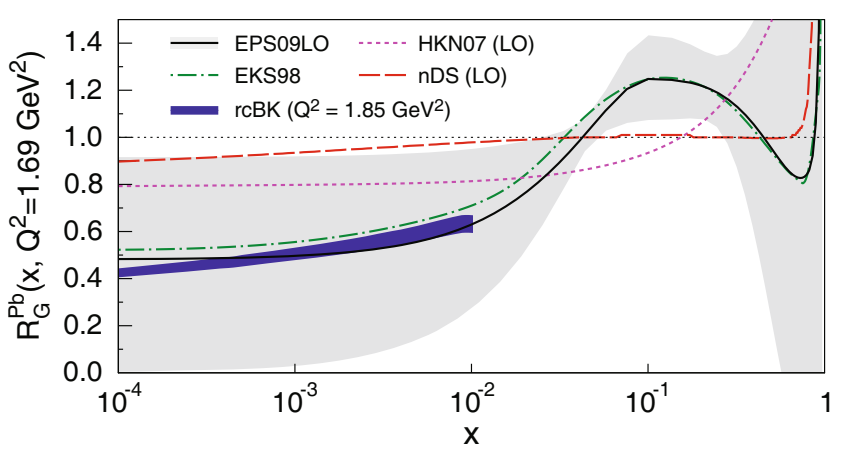

Fig. 42. Theoretical predictions for $R_{G}\left(x, Q^{2}\right)$ plotted at $Q^{2}=1.69 \mathrm{GeV}^{2}$ for a $P b$ nucleus: the models corresponding to different curves are explained in the plot legend. The models are: EPS09 [176], EKS 98 [177] (based on the leading-order (LO) global DGLAP analysis), HKN 07 [178], nDS [179] (nextto-leading-order (NLO) DGLAP analysis), and rcBK [165], plotted for $Q^{2}=1.85 \mathrm{GeV}^{2}$ (based on BK non-linear evolution with the running-coupling corrections (rcBK) [180-183], referred to as Model-II in sect. 3.2.1). The light-gray shaded area depicts the uncertainty band of EPS09, while the blue shaded area indicates the uncertainty band of the rcBK approach.

for the two structure functions, where the superscripts $p$ and $A$ label the structure functions for the protons and nuclei correspondingly. Ratios like those in eq. (23) can be constructed for the quark and gluon nuclear PDFs too. The ratio for the gluon distribution compares the number of gluons per nucleon in the nucleus to the number of gluons in a single free proton. Since the structure function $F_{L}$ measures the gluon distribution $x G\left(x, Q^{2}\right)[12,175]$, the ratio $R_{L}\left(x, Q^{2}\right)$ is close to the ratio $R_{G}\left(x, Q^{2}\right)$ of the gluon PDFs in the nucleus and the proton normalized the same way,

$$
R_{G}\left(x, Q^{2}\right) \equiv \frac{x G_{A}\left(x, Q^{2}\right)}{A x G_{p}\left(x, Q^{2}\right)} .
$$

A sample of theoretical predictions for the ratio $R_{G}\left(x, Q^{2}\right)$ for the gluon PDFs is plotted in fig. 42, comprising several DGLAP-based models along with the saturation-based prediction. Note that the DGLAP equation, describing evolution in $Q^{2}$, cannot predict the $x$ dependence of distribution functions at low $x$ without the data at comparable values of $x$ and at lower $Q^{2}$ : hence the DGLAP-based "predictions" in fig. 42 strongly suffer from the uncertainty in various ad hoc parameterizations of the initial conditions for DGLAP evolution. Conversely, the saturation prediction is based on the BK equation (19), which is an evolution equation in $x$, generating a very specific $x$-dependence of the distribution functions that follows from QCD: this leads to a narrow error band for the saturation prediction.

All existing approaches predict that the ratio $R_{G}$ would be below one at small $x$ : this is the nuclear shadowing phenomenon [184], indicating that the number of small- $x$ gluons per nucleon in a nucleus is lower than that in a free proton. In the DGLAP-based description of nuclear PDFs, shadowing is included in the parameteriza- 

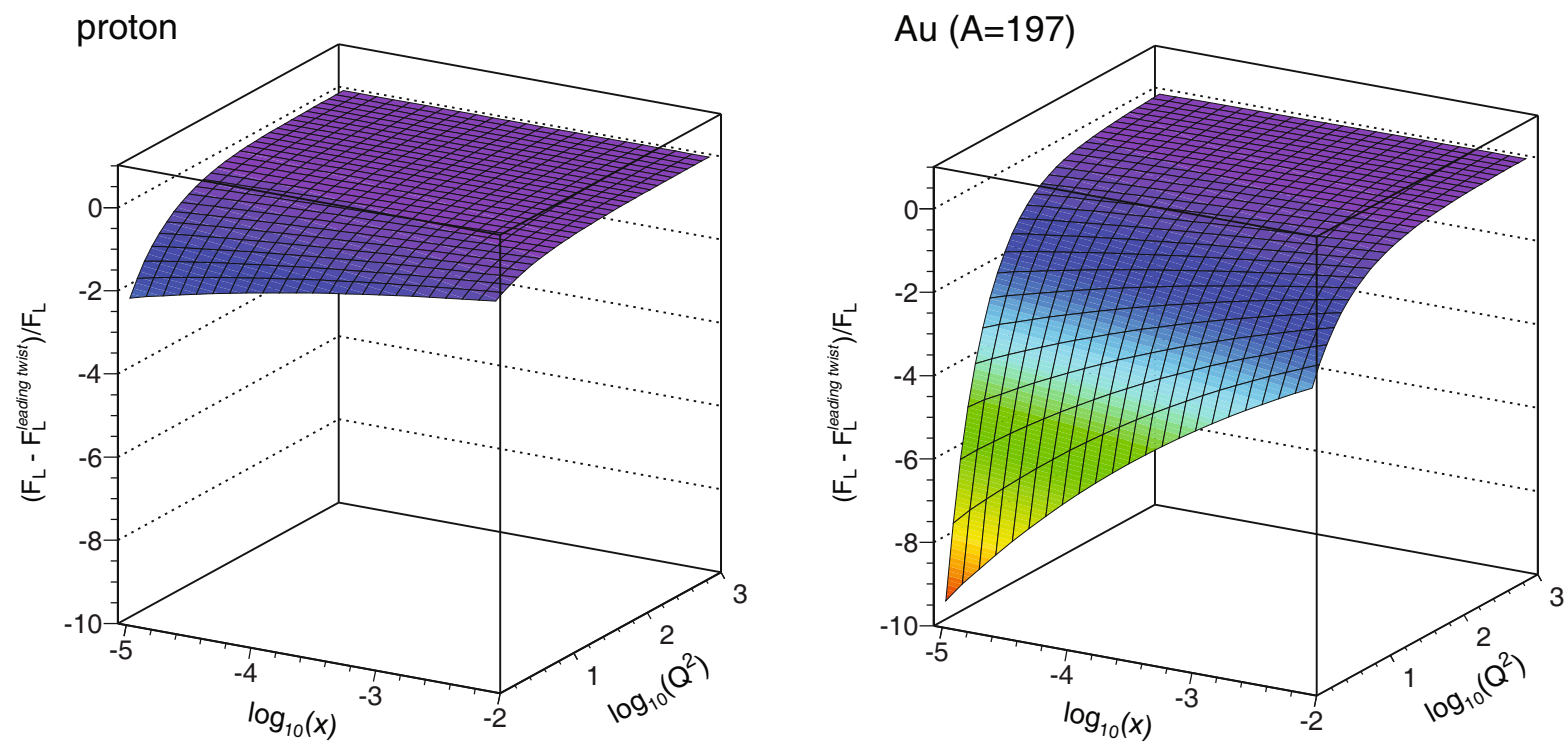

Fig. 43. Plots of the ratio from eq. (26) for $e+p$ and $e+$ Au scattering from [186], demonstrating the sensitivity of the nuclear structure function, $F_{L}$, to the higher-twist effects. The plots go down to $x=10^{-5}$ as the smallest $x$ reachable at an EIC (see fig. 32).

tions of the initial conditions for DGLAP evolution. In the saturation/CGC approach, gluon mergers and interactions dynamically lead to the decrease in the number of gluons (and other partons) per nucleon as compared to that in a single proton: this results in the shadowing of PDFs and reduction of structure functions as well.

One can clearly see from fig. 42 that new data is desperately needed to constrain the DGLAP-based prediction and/or to test the prediction of saturation physics. It is also clear that such data would eliminate some of the predictions shown in fig. 42 , allowing us to get closer to finding the model describing the correct physics. Still, as one can infer from fig. 42 , due to the multitude of theoretical predictions, the $R_{G}$ (or $R_{L}$ ) measurement alone may only rule out some of them, leaving several predictions in agreement with the data within the experimental error bars. As we detail further in sect. 3.2.2, one would need other measurements, like measurements of $R_{2}, F_{2}^{A}$, $F_{L}^{A}$, along with those described below in sect. 3.2 .2 , to uniquely determine the physics involved in high-energy DIS on the nucleus.

Nuclear effects in the structure functions can also be quantified using their expansion in powers of $1 / Q^{2}[185]$. The standard linear perturbative QCD approaches calculate the leading term in $1 / Q^{2}$ expansion of structure functions, the order-1 contribution, referred to as the "leading twist" term. The multiple re-scatterings of sect. 3.2.1 along with the gluon mergers of sect. 3.2.1 contribute to all orders in the $1 / Q^{2}$ expansion. Of particular interest is their contribution to the non-leading powers of $1 / Q^{2}$, known as "higher twists": the main parts of those corrections are enhanced by the nuclear "oomph" factor $A^{1 / 3}$ and by a power of $(1 / x)^{\lambda}$, coming in as

$$
\sim \frac{\Lambda_{Q C D}^{2} A^{1 / 3}}{Q^{2}}\left(\frac{1}{x}\right)^{\lambda} .
$$

We see that the telltale sign of saturation physics are the higher-twist corrections, which are enhanced in DIS on a nucleus, and at smaller $x$ (see footnote ${ }^{10}$ ).

To illustrate the effect of higher-twist corrections on the nuclear structure function we plot their relative contribution to $F_{L}$ defined by

$$
\frac{F_{L}-F_{L}(\text { leadingtwist })}{F_{L}}
$$

in fig. 43 as a function of $x$ and $Q^{2}$ as expected in the framework of the saturation-inspired Golec-BiernatWusthoff (GBW) model [168,187], which has been quite successful in describing the HERA $e+p$ data. The left panel of fig. 43 is for $e+p$ scattering, while the right one is for $e+\mathrm{Au}$. Note that the ratio is negative in both plots, indicating that higher twists tend to decrease the structure function. It is also clear from both plots that the effect of higher twists becomes stronger at smaller $x$, as expected from eq. (25). Comparing the two panels in fig. 43, we see that the higher-twist effects are also stronger in $e+\mathrm{Au}$ scattering due to nuclear enhancement. Figure 43 demonstrates that the structure function $F_{L}$ is rather sensitive to parton saturation. Experimentally, it is impossible to single out the higher-twist contribution if the $Q^{2}$ of interest is too high, making it difficult to plot the ratio from eq. (26) to verify the prediction in fig. 43 . At lower $Q^{2}$, experimental separation of the leading twist contribution from the higher-twist terms may also become a problem. Theoretical work is currently under way to enable the separation of higher twist terms in $F_{L}$ (and $F_{2}$ ), which is likely to make the ratio (26) an observable which could be measured at an EIC.

${ }^{10}$ In fact, equating the correction in eq. (25) to the leadingtwist order-1 term gives the saturation scale of eq. (20) as the value of $Q^{2}$ at which the higher-twist corrections become important. 

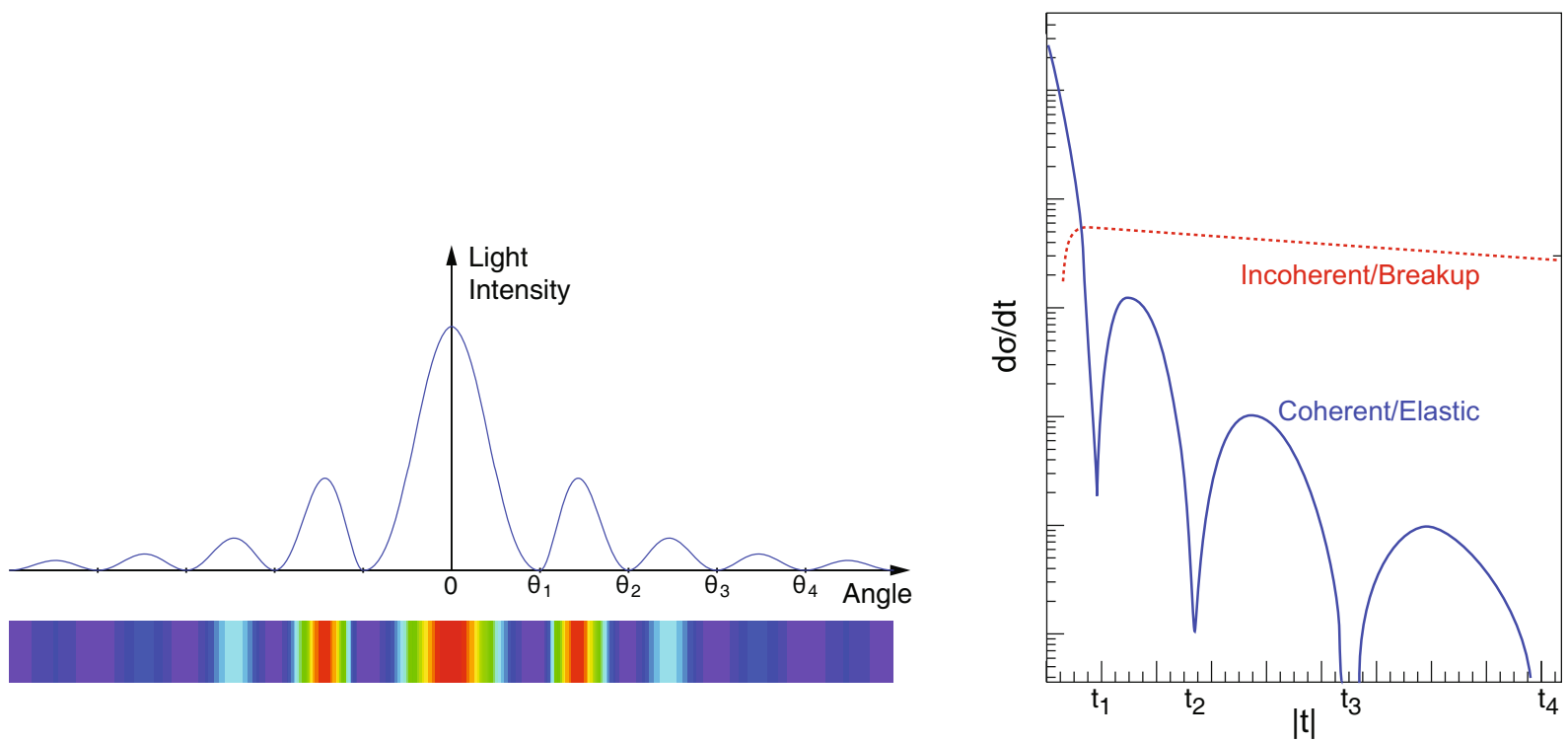

Fig. 44. Left panel: the diffractive pattern of light on a circular obstacle in wave optics. Right panel: the diffractive cross-section in high-energy scattering. The elastic cross-section in the right panel is analogous to the diffractive pattern in the left panel if we identify $|t| \approx k^{2} \theta^{2}$.

\section{Diffractive physics}

The phenomenon of diffraction is familiar to us from many areas of physics and is generally understood to arise from the constructive or destructive interference of waves. Perhaps the best analogy of diffraction in high-energy QCD comes from optics: imagine a standard example of a plane monochromatic wave with the wave number $k$ incident on a circular screen of radius $R$ (an obstacle). The diffractive pattern of the light intensity on a plane screen behind the circular obstacle is shown in the left panel of fig. 44 as a function of the deflection angle $\theta$, and features the well-known diffractive maxima and minima. The positions of the diffractive minima are related to the size of the obstacle by $\theta_{i} \sim 1 /(k R)$ for small-angle diffraction.

Elastic scattering in QCD has a similar structure. Imagine a hadron (a projectile) scattering on a target nucleus. If the scattering is elastic, both the hadron and the nucleus will be intact after the collision. The elastic process is described by the differential scattering cross-section $\mathrm{d} \sigma_{e l} / \mathrm{d} t$ with the Mandelstam variable $t$ describing the momentum transfer between the target and the projectile. A typical $\mathrm{d} \sigma_{e l} / \mathrm{d} t$ is sketched by the solid line in the right panel of fig. 44 as a function of $t$. Identifying the projectile hadron with the incident plane wave in the wave optics example, the target nucleus with the obstacle, and writing $|t| \approx k^{2} \theta^{2}$ valid for small angles, we can see that the two panels of fig. 44 exhibit analogous diffractive patterns and, therefore, describe very similar physics! The minima (and maxima) of the cross-section $\mathrm{d} \sigma_{e l} / \mathrm{d} t$ in the right panel of fig. 44 are also related to the inverse size of the target squared, $\left|t_{i}\right| \sim 1 / R^{2}$. This is exactly the same principle as employed for spatial imaging of the nucleons as described in sect. 2.3.
The essential difference between QCD and wave optics is summarized by two facts: i) The proton/nuclear target is not always an opaque "black disk" obstacle of geometric optics. A smaller projectile, which interacts more weakly due to color-screening and asymptotic freedom, is likely to produce a different diffractive pattern from the larger, more strongly interacting, projectile. ii) The scattering in QCD does not have to be completely elastic: the projectile or target may break up. The event is still called diffractive if there is a rapidity gap, as described in Sidebar V. The cross-section for the target breakup (leaving the projectile intact) is plotted by the dotted line in the right panel of fig. 44, and does not exhibit the diffractive minima and maxima.

The property i) is very important for diffraction in DIS in relation to saturation/CGC physics. As we have seen above, owing to the uncertainty principle, at higher $Q^{2}$, the virtual photon probes shorter transverse distances, and is less sensitive to saturation effects. Conversely, the virtual photon in DIS with the lower $Q^{2}$ is likely to be more sensitive to saturation physics. Due to the presence of a rapidity gap, the diffractive cross-section can be thought of as arising from an exchange of several partons with zero net color between the target and the projectile (see Sidebar V). In high-energy scattering, which is dominated by gluons, this color neutral exchange (at the lowest order) consists of at least two exchanged gluons. We see that compared to the total DIS cross-section, which can be mediated by a single gluon or quark exchange, the diffractive cross-section includes more interactions, and, therefore, is likely to be more sensitive to saturation phenomena, which, at least in the MV model, are dominated by multiple re-scatterings. In fact, some diffractive processes are related to the square of the gluon distribution $x G$. We conclude that the diffractive cross-section is likely to be a very sensitive test of saturation physics. 
Of particular interest is the process of elastic vector meson $(V)$ production, $e+\mathrm{A} \rightarrow e+V+\mathrm{A}$. The crosssection $\mathrm{d} \sigma / \mathrm{d} t$ for such processes at lower $Q^{2}$ is sensitive to the effects of parton saturation [188], as we will explicitly demonstrate below. For a vector meson with a sufficiently spread-out wave function (a large meson, like $\phi$ or $\rho)$, varying $Q^{2}$ would allow one to detect the onset of the saturation phenomenon [188].

Diffraction can serve as a trigger of the onset of the black disk limit of eq. (18). In that regime, the total diffractive cross-section $\sigma_{\text {diff }}$ (including all the events with rapidity gaps), would constitute $50 \%$ of the total crosssection,

$$
\frac{\sigma_{\text {diff }}}{\sigma_{\text {tot }}}=\frac{1}{2} .
$$

This may sound counterintuitive: indeed, the naive expectation in QCD is that events with gaps in rapidity are exponentially suppressed. It was therefore surprising to see that a large fraction (approximately 15\%) of all events reported by HERA experiments are rapidity gap events [189]. This corresponds to a situation where the projectile electron slams into the proton at rest with an energy 50000 times the proton rest energy and in about 1 in 7 such scatterings, nothing happens to the proton. In the black disk regime this ratio should increase to 1 in 2 events.

\subsubsection{Key measurements}

The main goal of the $e+$ A program at an EIC is to unveil the collective behavior of densely packed gluons under conditions where their self-interactions dominate, a regime where non-linear QCD supersedes "conventional" linear QCD. The plain fact that there is no data from this realm of the nuclear wave function available is already a compelling enough reason to build an EIC. It is truly terra incognita. However, our goal is not only to observe the onset of saturation, but to explore its properties and reveal its dynamical behavior. As explained above, the saturation scale squared for nuclei includes an "oomph" factor of $A^{1 / 3}$ making it larger than in the proton (cf. eq. (22)); fig. 45 demonstrates that. While at an EIC, a direct study of the saturation region in the proton is impossible (while remaining in the perturbative QCD region where the coupling $\alpha_{s}$ is small, i.e., above the horizontal dashed line in the figure), this $A^{1 / 3}$ enhancement may allow us to study the saturation region of large nuclei, such as gold $(\mathrm{Au})$. In fig. 45, the borders of the kinematic reach of the EIC are indicated by the diagonal black lines corresponding to different combinations of electron and hadron beam energies; the actual kinematic reach regions are to the right of the border lines.

A wide range of measurements with an EIC can distinguish between predictions in the CGC, or other novel frameworks, and those following from the established DGLAP evolution equations. However, these comparisons have to be made with care. Non-linear models are valid only at or below the saturation scale, $Q_{s}^{2}$, while perturbative QCD (pQCD) based on the linear DGLAP evolution equation is strictly only applicable at large $Q^{2}$. In

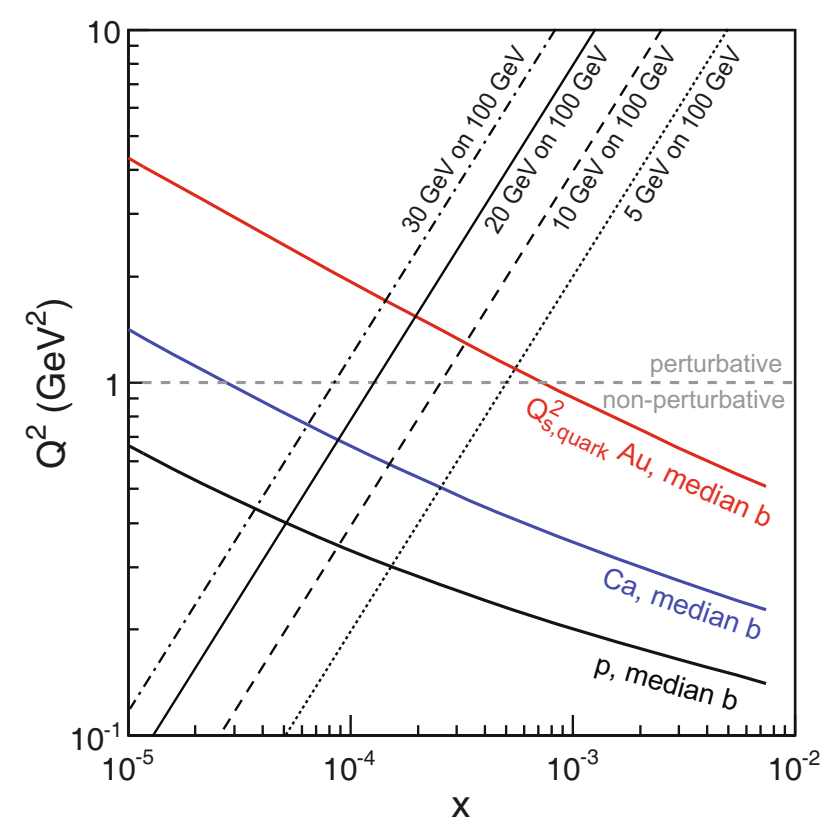

Fig. 45. The kinematic reach in $x$ and $Q^{2}$ of the EIC for different electron beam energies, given by the regions to the right of the diagonal black lines, compared with predictions of the saturation scale, $Q_{s}$, in $p, \mathrm{Ca}$, and $\mathrm{Au}$ from Model-I (see sect. 3.2.1 and note that $x<0.01$ in the figure).

the range $Q^{2}<Q_{s}^{2}$, solely non-linear theories such as the CGC can provide quantitative calculations. It is only in a small window of approximately $1 \lesssim Q^{2} \lesssim 4 \mathrm{GeV}^{2}$ where a comparison between the two approaches can be made (see fig. 45). Due to the complexity of high-energy nuclear physics, at the end, the final insight will come from the thorough comparison of models calculations with a multitude of measurements, each investigating different aspects of the low- $x$ regime. We will learn from varying the ion species, A, from light to heavy nuclei, studying the $Q^{2}, x$, and $t$ dependence of the cross-section in inclusive, semi-inclusive, and exclusive measurements in DIS and diffractive events.

In what follows we discuss a small set of key measurements whose ability to extract novel physics is beyond question. They serve primarily to exemplify the very rich physics program available at an EIC. These "golden" measurements are summarized in table 4 with two EIC energy options. These measurements are discussed in further detail in the remainder of this section. It should be stressed that the low- $x$ physics program will only reach its full potential when the beam energies are large enough to reach sufficiently deep into the saturation regime. Ultimately this will only be possible at an EIC where $x \sim 10^{-4}$ can be reached at $Q^{2}$ values of $1-2 \mathrm{GeV}^{2}$ as indicated in fig. 45 . Only the highest energies will give us enough of a lever arm in $Q^{2}$ to study the crossing into the saturation region allowing us to, at the same time, make the comparison with DGLAP-based pQCD and CGC predictions. The statistical error bars depicted in the figures described in this section are derived by assuming an integrated luminosity of $\int \mathcal{L} \mathrm{d} t=10 \mathrm{fb}^{-1} / \mathrm{A}$ for each species and include exper- 
Table 4. Key measurements in $e+$ A collisions at an EIC with two energy options, as shown in fig. 32, addressing the physics of high gluon densities.

\begin{tabular}{|c|c|c|c|c|}
\hline Deliverables & Observables & What we learn & Low-energy option & High-energy option \\
\hline $\begin{array}{c}\text { Integrated gluon } \\
\quad \text { momentum } \\
\text { distributions } G_{A}\left(x, Q^{2}\right)\end{array}$ & $F_{2}, F_{L}$, and $F_{2}^{c \bar{c}}$ & $\begin{array}{l}\text { Nuclear wave } \\
\text { function; } \\
\text { saturation }\end{array}$ & $\begin{array}{c}\text { Gluons at } \\
10^{-3} \lesssim x \lesssim 1\end{array}$ & $\begin{array}{c}\text { Exploration } \\
\text { of the saturation } \\
\text { regime }\end{array}$ \\
\hline $\begin{array}{c}k_{T} \text {-dependent } \\
\text { gluons } f\left(x, k_{T}\right) ; \\
\text { gluon correlations }\end{array}$ & $\begin{array}{l}\text { Di-hadron } \\
\text { correlations }\end{array}$ & $\begin{array}{c}\text { Non-linear QCD } \\
\text { evolution/universality; } \\
\text { saturation scale } Q_{s}\end{array}$ & $\begin{array}{c}\text { Onset of } \\
\text { saturation; } \\
Q_{s} \text { measurement }\end{array}$ & $\begin{array}{l}\text { Non-linear } \\
\text { small- } x \\
\text { evolution }\end{array}$ \\
\hline $\begin{array}{c}\text { Spatial gluon } \\
\text { distributions } f\left(x, b_{T}\right) \\
\text { gluon correlations }\end{array}$ & $\begin{array}{l}\text { Diffractive dissociation } \\
\qquad \sigma_{\text {diff }} / \sigma_{\text {tot }} \\
\text { vector mesons \& DVCS } \\
\qquad \mathrm{d} \sigma / \mathrm{d} t, \mathrm{~d} \sigma / \mathrm{d} Q^{2}\end{array}$ & $\begin{array}{l}\text { Non-linear small- } x \\
\text { evolution; } \\
\text { saturation dynamics; } \\
\text { black disk limit }\end{array}$ & $\begin{array}{c}\text { saturation } \\
\text { vs. non-saturation } \\
\text { models }\end{array}$ & $\begin{array}{c}\text { Spatial } \\
\text { gluon } \\
\text { distribution; } \\
Q_{s} \text { vs. centrality }\end{array}$ \\
\hline
\end{tabular}

imental cuts (acceptance and momentum). Systematical uncertainties were estimated in a few cases based on experience from HERA. Ultimately they will depend on the details of detectors and machine and hence cannot be fully addressed at this time.

\section{Structure functions}

As we mentioned above in sect. 3.2.1, the differential unpolarized cross-section for DIS is fully described by a set of basic kinematic variables and two structure functions, $F_{2}\left(x, Q^{2}\right)$ and $F_{L}\left(x, Q^{2}\right)$, that encapsulate the rich structure of valence quarks, sea quarks and anti-quarks $\left(F_{2}\right)$ and gluons $\left(F_{L}\right)$. The structure function $F_{L}$ is directly proportional to the gluon distribution function, $F_{L}\left(x, Q^{2}\right) \propto \alpha_{s} x G\left(x, Q^{2}\right)$, at low $x$ and not very small $Q^{2}[12,175]$. A precise knowledge of $F_{L}$ is mandatory for the study of gluons and their dynamics in nucleons and nuclei (see Sidebar II).

As demonstrated in sect. 3.2.1 and shown in fig. 42, various models have different predictions for the gluon distribution ratio $R_{G}\left(x, Q^{2}\right)$. The same is true for the ratios $R_{2}\left(x, Q^{2}\right)$ and $R_{L}\left(x, Q^{2}\right)$, along with the nuclear structure functions $F_{2}^{A}\left(x, Q^{2}\right)$ and $F_{L}^{A}\left(x, Q^{2}\right)$. These observables can be measured at the EIC as functions of $x, Q^{2}$, and $A$. (For the $A$-dependence one will need to perform machine runs with different types of nuclei, while to extract $F_{L}$ one needs to vary the center-of-mass energy.) The multitude of theoretical predictions should be counterbalanced by the multitude of possible data points for the four observables in the 3 -dimensional $\left(x, Q^{2}, A\right)$ parameter space. It is possible that the abundance of data obtained with sufficient statistics would allow one to rule out many models, hopefully pinpointing the one that best describes all the data to be obtained.

In order to verify the EIC's capability to measure the structure functions $F_{2}$ and $F_{L}$, we conducted simulation of inclusive events in $e+\mathrm{Au}$ collisions using PYTHIA with EPS09 nuclear parton distribution functions [176]. Figure 46 shows the resulting structure functions $F_{2}$ (left) and $F_{L}$ (right) as functions of $Q^{2}$ with their respective $x$ values. The curves and error bands for $F_{2}$ derived from the EPS09 distribution function in NLO $[176,190]$ are overlaid. The comparison of the current EPS09 uncertainty bands with the errors of the respective data points demonstrates that for $x \lesssim 0.01$, the EIC will have a substantial impact on reducing the uncertainty of leading-twist shadowing models.

Any measurement of $F_{L}$ requires data at a wide range of $\sqrt{s}$. In our $F_{L}$ studies presented on the right in fig. 46, we varied the beam energies over the range indicated in the panel. The final values for $F_{L}$ were extracted using the standard Rosenbluth method. This method is extremely sensitive to the quality of the absolute normalization achieved at the various energies. Since systematic uncertainties depend on the quality of the final detectors and on the accuracy of luminosity measurements, their ultimate magnitude is hard to estimate. In our studies we assumed systemic normalization uncertainties of $3 \%$ per energy, the same as the values that were achieved at HERA. The presented errors include both systematical and statistical contributions.

A comparison of $F_{2}$ and $F_{L}$ clearly shows the intricacy of the $F_{L}$ studies. While $F_{L}$ is of enormous importance for the study of gluons, its measurement is very difficult. In addition, the kinematic reach of $F_{L}$ measurements is much narrower than that of $F_{2}$.

An alternative and complementary method for studying the gluon density is via the charm structure function $F_{2}^{c \bar{c}}$. The left plot in fig. 47 shows $F_{2}^{c \bar{c}}$ versus $Q^{2}$ for various $x$-values in $e+\mathrm{Au}$ collisions at an EIC. Also shown are curves and respective uncertainty bands resulting from the EPS09 parameterization of nuclear parton distribution functions $[176,190]$. While an EIC will certainly constrain these leading-twist shadowing models further for $x \lesssim 5 \times 10^{-3}$, it appears that the improvement would be rather modest. Here, one has to keep in mind that through the charm structure function, one probes the PDFs at a somewhat higher value of Bjorken $x$, namely at $x^{\prime} \approx x\left(1+\left(4 m_{c}^{2}\right) / Q^{2}\right)$, where the PDFs are better constrained by the existing data. The fact that $F_{2}^{c \bar{c}}$ is so surprisingly well-predicted in DGLAP-based approaches compared to $F_{L}$ can be used to test for differences between the traditional leading-twist shadowing models (such as EPS09) and models that involve non-linear dynamics. 

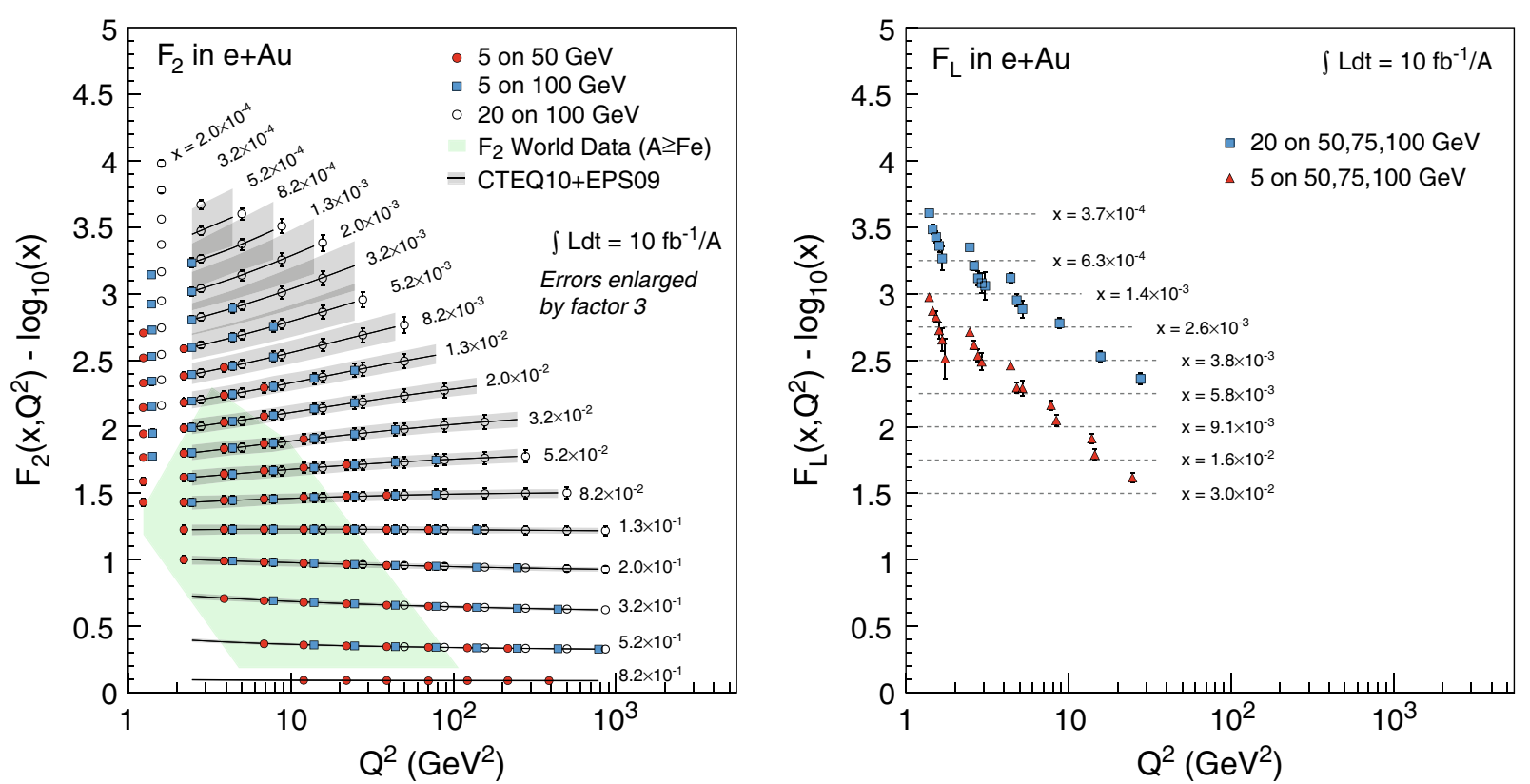

Fig. 46. The structure functions $F_{2}$ (left) and $F_{L}$ (right) as functions of $Q^{2}$ for various $x$ values in $e+$ Au collisions at an EIC generated by using PYTHIA with EPS09 nuclear PDFs [176]. $F_{2}$ and $F_{L}$ are offset by $\log _{10}(x)$ for clarity. Measurements and corresponding errors at different energies (indicated in the panels) are presented and illustrate the respective kinematic reach. Data points from different energies at the same $Q^{2}$ are slightly offset along the abscissa for visibility where necessary. Statistical errors for $F_{2}$ and $F_{L}$ are based on $10 \mathrm{fb}^{-1} / \mathrm{A}$ integrated luminosity for the sum of all measurements at all indicated energies. Both for $F_{2}$ and $F_{L}$ we assumed a $3 \%$ systematic uncertainty and added it to the statistical errors in quadrature; for $F_{2}$ the combined errors are scaled up by a factor of 3 to make them visible. For $F_{2}$, we also depict the curves and respective uncertainty bands from the EPS09 parameterization of the nuclear parton distribution functions $[176,190]$. The green shaded area indicates the $\left(Q^{2}, x\right)$ range of existing measurements for nuclei larger than iron, demonstrating the kinematic reach of an EIC.
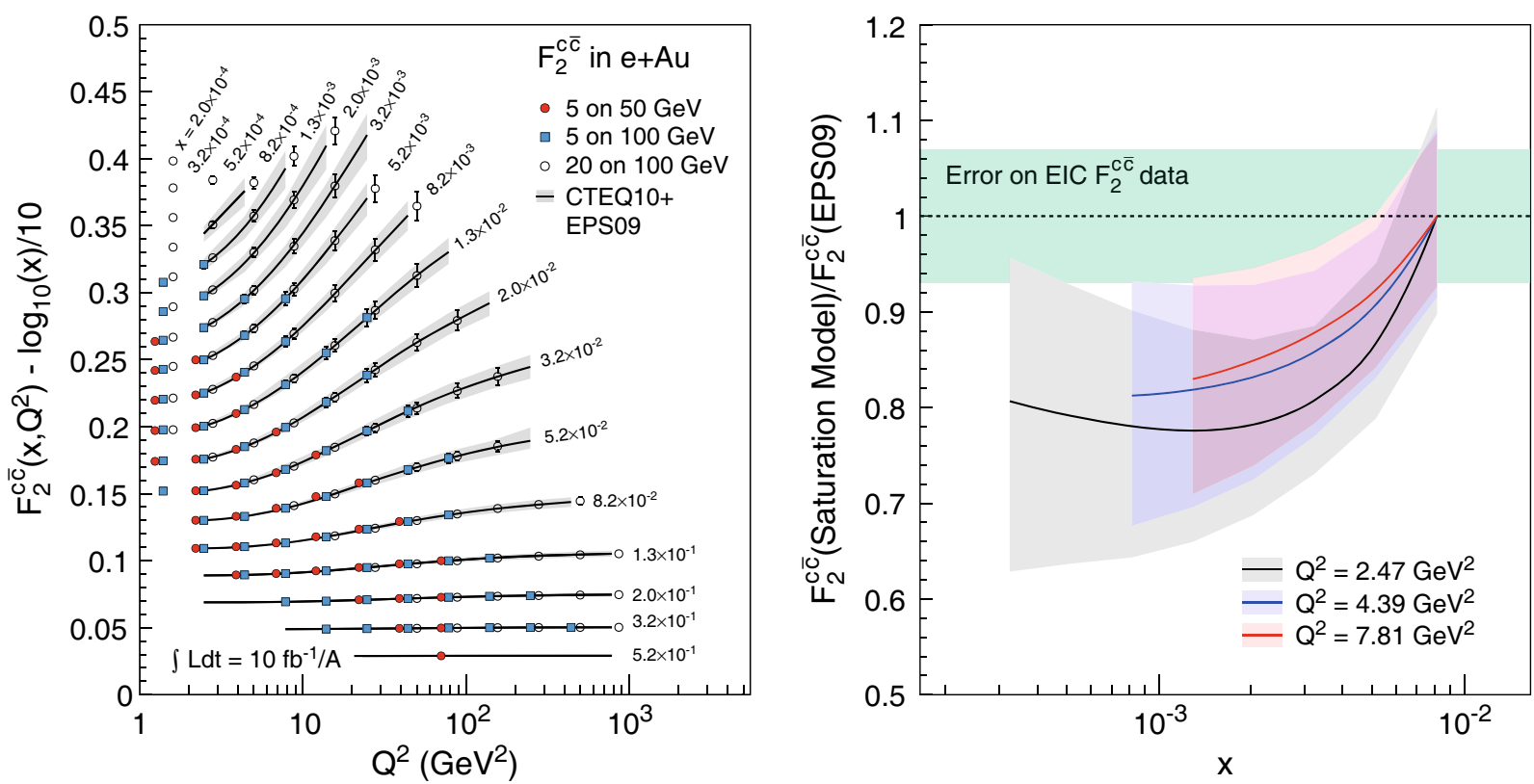

Fig. 47. Left panel: the charm structure function $F_{2}^{c \bar{c}}$ versus $Q^{2}$ for various $x$ values in $e+$ Au collisions at an EIC generated by using PYTHIA with EPS09 nuclear PDFs [176]. $F_{2}^{c \bar{c}}$ values are offset by $\log _{10}(x) / 10$ for clarity. Measurements and corresponding errors for three different energies are presented and illustrate the respective kinematic reach. Data points from different energies at the same $Q^{2}$ and $x$ are slightly offset along the abscissa for better visibility. Statistical errors are based on $10 \mathrm{fb}^{-1} / \mathrm{A}$ integrated luminosity for the sum of all three energies. The depicted errors are derived from the statistical errors and a $7 \%$ systematic uncertainty added in quadrature. Also shown are curves and respective uncertainty bands from the EPS09 parameterization of the nuclear parton distribution functions [176,190]. Right panel: ratio of $F_{2}^{c \bar{c}}$ predictions from a saturation model (rcBK) [165] and EPS09 for three different $Q^{2}$ values. The uncertainty band for each $Q^{2}$ value reflects the combined uncertainties in both models. The green band depicts the approximate uncertainties of EIC measurements of $F_{2}^{c \bar{c}}$ thus indicating in what kinematic range an EIC will be able to distinguish between traditional leading-twist shadowing and saturation models. 

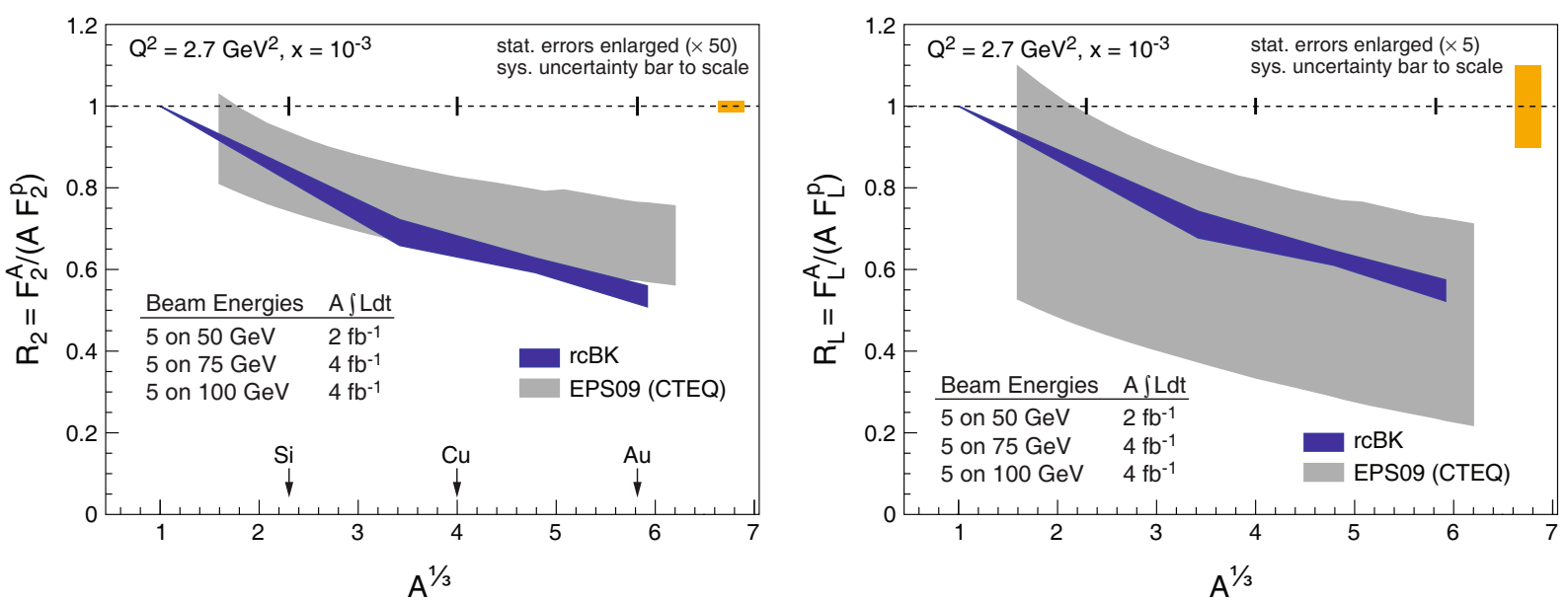

Fig. 48. Left: the ratio $R_{2}$ of the $F_{2}$ structure function in a nucleus over that of the proton scaled by mass number $A$ as a function of $A^{1 / 3}$. The predictions from a CGC based calculation (rcBK) [165] and from a linear evolution using the latest nuclear PDFs (EPS09) and CTEQ6 for the proton are shown $[176,190]$. Right: the same for the longitudinal structure function $F_{L}$ (see text for details).

The right plot in fig. 47 compares one such model, the rcBK model, to EPS09 by depicting the ratio of these models predictions for $F_{2}^{c \bar{c}}$ for three different $Q^{2}$ values as functions of $x$ where we expect these non-linear dynamics to be important. rcBK is a saturation model in the CGC framework based on the BK non-linear evolution with the running-coupling corrections [165, 180-182]: we referred to it as Model-II in sect. 3.2.1. As follows from the right plot in fig. 47 , it predicts a markedly different $x$-dependence than NLO pQCD calculations based on EPS09: importantly, the difference between the models (together with the combined uncertainty of both models) exceeds the expected uncertainty of EIC measurements (the green band). It appears that with a sufficient experimental effort the EIC would be able to distinguish between the saturation and leading-twist shadowing predictions for $F_{2}^{c \bar{c}}$, providing us with another measurement capable of identifying saturation dynamics.

Clearly, the EIC will reach into unexplored regions with unprecedented precision and will be able to distinguish between traditional and non-linear QCD models. These measurements will have a profound impact on our knowledge of nuclear structure functions and the underlying evolution scheme, likely allowing to rule out many theoretical models and to establish the correct underlying physics. For a better discrimination between models, especially involving non-linear dynamics, several observables sensitive to the gluon distribution will be essential: i) scaling violation of $F_{2}$, ii) the direct measurement of $F_{L}$, and iii) $F_{2}^{c \bar{c}}$.

Note that all three observables can be measured already at moderate luminosities with good statistical precision. The final experimental errors for the structure functions to be measured at EIC will be dominated by systematic uncertainties. High luminosities are not required for the measurement of structure functions, while precise knowledge of the actual luminosity is paramount.

In the context of model comparisons, it is important to note that DGLAP-based models can not predict the $A$-dependence of PDFs and structure functions without making additional data-driven assumptions: this is the ori- gin of the broad error bars of the EPS09 model in fig. 42. However, this broad error band may also be indicative of the ability of such models to indiscriminately describe a broad range of $F_{2}$ and $F_{L}$ data: in such cases, further experimental tests of DGLAP-based approaches can be carried out using other observables described in the sections below.

To further illustrate this point, we show in fig. 48 two theoretical predictions for the ratio $R_{2}\left(R_{L}\right)$, i.e., the ratio of the $F_{2}\left(F_{L}\right)$ structure function in a nucleus over that of the proton scaled by mass number $A$. The calculations are shown as a function of $A^{1 / 3}$ at $Q^{2}=2.7 \mathrm{GeV}^{2}$ and $x=10^{-3}$. In the absence of any nuclear effects, both ratios $R_{2}$ and $R_{L}$ should be unity. Due to the lack of precise $e+\mathrm{A}$ data, the models are not strongly constrained and we use error bands to indicate the range of the referring predictions. In fig. 48 we depict two calculations for $R_{2}$ (left) and $R_{L}$ (right). The calculation shown in blue is based on the CGC framework (rcBK) [165] which was already discussed earlier. It features an approximate $A^{1 / 3}$ scaling of the saturation scale squared (see sect. 3.2.1), which allows us to make reasonably precise predictions for $R_{2}$ and $R_{L}$; the second calculation (gray band) uses the linear NLO DGLAP evolution in pQCD resulting in the nuclear parton distribution set EPS09 $[176,190]$ : it exhibits a broader error band, similar to the case of $R_{G}$ in fig. 42 . Even in linear DGLAP evolution, non-linear effects may be absorbed into the non-perturbative initial conditions for the nuclear PDFs, where the $A$-dependence is obtained through a fit to available data, resulting in the ability of DGLAP-based approaches to indiscriminately describe a broad range of nuclear data. This leads to the wide error bands of EPS09, especially for $F_{L}$, clearly demonstrating the lack of existing nuclear structure function data. Due to these large theoretical error bars, the measurements of $R_{2}$ and $R_{L}$ as functions of $A^{1 / 3}$, while significantly extending our knowledge of nuclear structure functions, may not allow one to directly distinguish between a non-linear (saturation) and linear (DGLAP) evolution approaches at an EIC with low collision energies. 

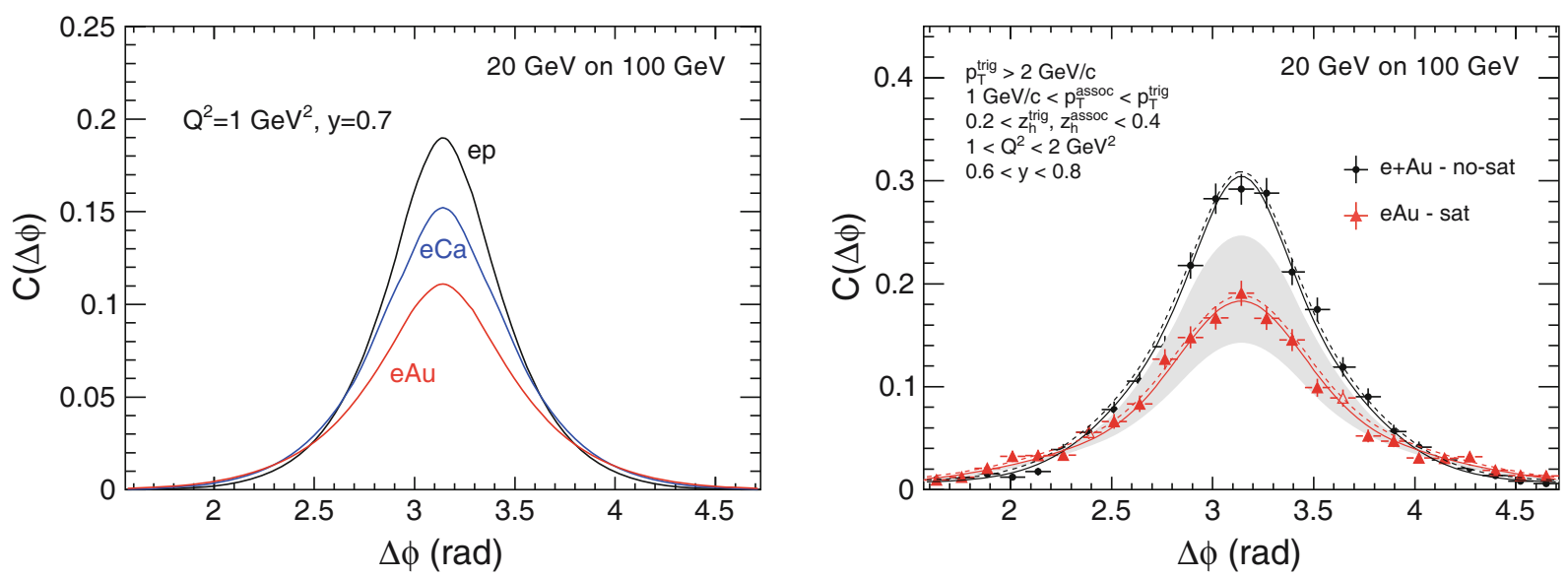

Fig. 49. Left: a saturation model prediction of the coincidence signal versus azimuthal angle difference $\Delta \varphi$ between two hadrons in $e+p, e+\mathrm{Ca}$, and $e+\mathrm{A}$ collisions [192-194]. Right: A comparison of saturation model prediction for $e+$ A collisions with calculations from conventional non-saturated model. Statistical error bars correspond to $1 \mathrm{fb}^{-1} / \mathrm{A}$ integrated luminosity.

Shown along the line at unity by vertical notches in fig. 48 are the statistical errors that were obtained from the Rosenbluth separation technique using the range of energies indicated in the figure. The statistical error bars were generated from a total of $10 \mathrm{fb}^{-1} / \mathrm{A}$ of Monte Carlo data, spread over three beam energies (see plot legend for details). The statistical error bars are scaled up by a factor of 50 for $R_{2}$ and a factor of 5 for $R_{L}$; as the statistical errors are clearly small, the experimental errors will be dominated by the systematic uncertainties shown by the orange bars drawn to scale in the two panels of fig. 48. This measurement, together with the ones described below, will constrain models to such an extent that the "true" underlying evolution scheme can be clearly identified. It is also possible that data from a lower-energy EIC would decrease the error band of DGLAP-based predictions, allowing for the $R_{2}$ and $R_{L}$ measurement at a higher energy EIC (smaller $x$ ) to discriminate between saturation and DGLAP approaches. However it is also possible that, on its own, the $R_{2}$ and $R_{L}$ measurements may turn out to be insufficient to uniquely differentiate DGLAP-based models with nuclear "shadowing" in the initial conditions from the saturation/CGC effects; in such a case, the measurements presented below along with $F_{2}^{c \bar{c}}$ shown above will be instrumental in making the distinction.

\section{Di-hadron correlations}

One of the experimentally easiest and compelling measurement in $e+\mathrm{A}$ is that of di-hadron azimuthal correlations in $e+\mathrm{A} \rightarrow e^{\prime}+h_{1}+h_{2}+X$ processes. These correlations are not only sensitive to the transverse-momentum dependence of the gluon distribution, but also to that of gluon correlations for which first principles CGC computations are only now becoming available. The precise measurements of these di-hadron correlations at an EIC would allow one to extract the spatial multi-gluon correlations and study their non-linear evolution. Saturation effects in this channel correspond to a progressive disappearance of the back-to-back correlations of hadrons with increasing atomic number $A$. These correlations are usually measured in the plane transverse to the beam axis (the "transverse plane"), and are plotted as a function of the azimuthal angle $\Delta \varphi$ between the momenta of the produced hadrons in that plane. Back-to-back correlations are manifested by a peak at $\Delta \varphi=\pi$ (see fig. 49). In the conventional pQCD picture, one expects from momentum conservation that the back-to-back peak will persist as one goes from $e+p$ to $e+\mathrm{A}$. In the saturation framework, due to multiple re-scatterings and multiple gluon emissions, the large transverse momentum of one hadron is balanced by the momenta of several other hadrons (instead of just one back-to-back hadron), effectively washing out the correlation at $\Delta \varphi=\pi[191]$. A comparison of the heights and widths of the di-hadron azimuthal distributions in $e+\mathrm{A}$ and $e+p$ collisions respectively would clearly mark out experimentally such an effect.

An analogous phenomenon has already been observed for di-hadrons produced at forward rapidity in comparing $d+\mathrm{Au}$ with $p+p$ collisions at RHIC (see sect. 3.4.1). In that case, di-hadron production is believed to proceed from valence quarks in the deuteron (proton) scattering on small- $x$ gluons in the target $\mathrm{Au}$ nucleons (proton). Lacking direct experimental control over $x$, the onset of the saturation regime is controlled by changing the centrality of the collision, the di-hadron rapidity and the transverse momenta of the produced particles. (Note that the gluon density and, consequently, the saturation scale $Q_{s}$ depend on the impact parameter and on rapidity/Bjorken $x$.) Experimentally, a striking flattening of the $\Delta \varphi=\pi$ peak in $d+\mathrm{Au}$ collisions as compared to $p+p$ collisions is observed in central collisions $[195,196]$, but the peak re-appears in peripheral collisions, in qualitative agreement with the CGC predictions, since saturation effects are stronger in central collisions.

There are several advantages to studying di-hadron correlations in $e+\mathrm{A}$ collisions versus $d+\mathrm{Au}$. Directly using a point-like electron probe, as opposed to a quark bound in a proton or deuteron, is extremely beneficial. It is experimentally much cleaner as there is no "specta- 

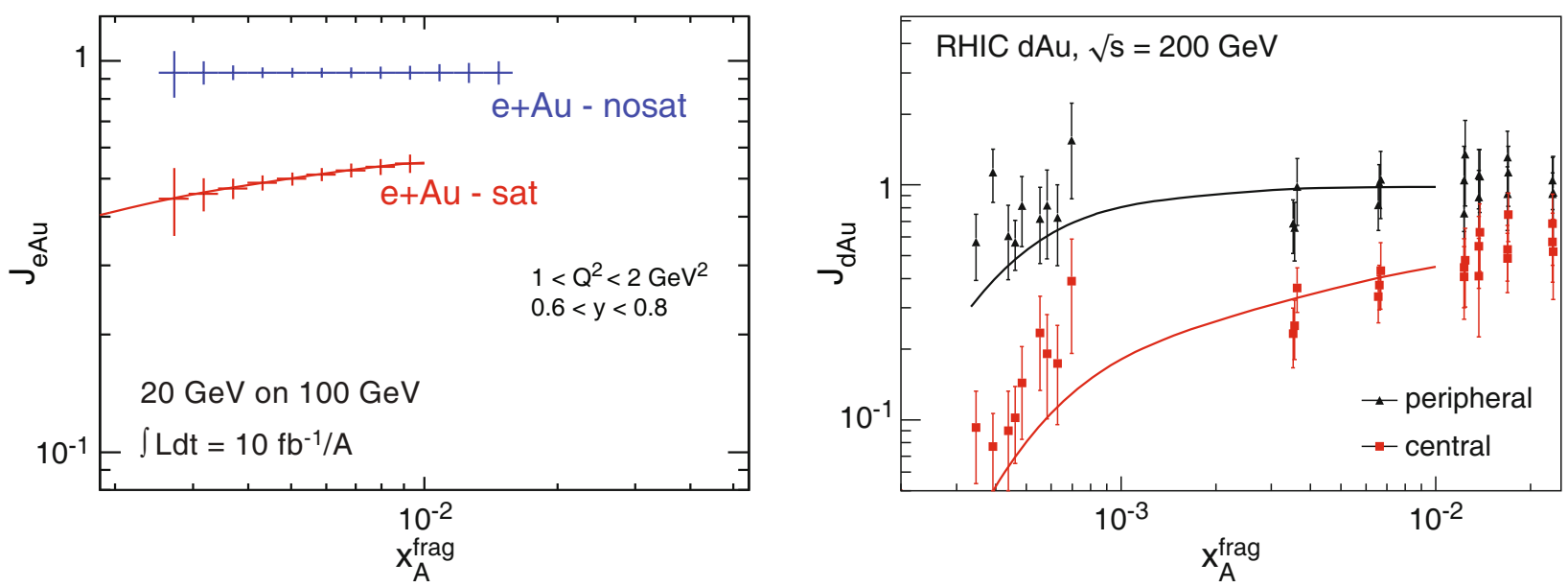

Fig. 50. Left: the relative yield of di-hadrons in $e+$ Au compared to $e+p$ collisions, $J_{e A u}$, plotted versus $x_{A}^{\text {frag }}$, which is an approximation of the longitudinal-momentum fraction of the probed gluon $x_{g}$. Predictions for linear (nosat) and non-linear (sat) QCD models are presented. The statistical error bars correspond to $10 \mathrm{fb}^{-1} / \mathrm{A}$ integrated luminosity. Right: the corresponding measurement in $\sqrt{s}=200 \mathrm{GeV}$ per nucleon $d+\mathrm{Au}$ collisions at RHIC [195]. The curves in both panels depict calculations in the CGC framework [192-194].

tor" background to subtract from the correlation function. The access to the exact kinematics of the DIS process at an EIC would allow for more accurate extraction of the physics than is possible at RHIC or the LHC. Because there is such a clear correspondence between the physics of this particular final state in $e+\mathrm{A}$ collisions to the same in $p+\mathrm{A}$ collisions, this measurement is an excellent testing ground for universality of multi-gluon correlations.

The left plot in fig. 49 shows prediction in the CGC framework for di-hadron $\Delta \varphi$ correlations in deep inelastic $e+p, e+\mathrm{Ca}$, and $e+\mathrm{Au}$ collisions [192-194]. The calculations are made for $Q^{2}=1 \mathrm{GeV}^{2}$ and include a Sudakov form factor to account for generated radiation through parton showers; only $\pi^{0}$ 's were used. The highest transverse momentum hadron in the di-hadron correlation function is called the "trigger" hadron, while the other hadron is referred to as the "associate" hadron. The "trigger" hadrons have transverse momenta of $p_{T}^{\text {trig }}>$ $2 \mathrm{GeV} / c$ and the "associate" hadrons were selected with $1 \mathrm{GeV} / c<p_{T}^{\text {assoc }}<p_{T}^{\text {trig }}$. The CGC-based calculations show a dramatic "melting" of the back-to-back correlation peak with increasing ion mass. The right plot in fig. 49 compares the prediction for $e+\mathrm{A}$ with a conventional non-saturated correlation function. The latter was generated by a hybrid Monte Carlo generator, consisting of PYTHIA-6 [197] for parton generation, showering and fragmentation and DPMJet-III [198] for the nuclear geometry, and a cold matter energy-loss afterburner [199]. The EPS09 [176] nuclear parton distributions were used to include leading twist shadowing. The resulting correlation function is shown in the right panel of fig. 49 by the black solid and dashed lines. The solid black curve includes detector smearing effects, while the dashed curve shows the result without taking into account any detector response. The red curve in the right panel of fig. 49 represents the CGC predictions. While the underlying model is identical to that shown in the left panel of fig. 49, the simulations include all charged hadrons as well as the quark channel contributions. The solid and dashed red lines represent detector response effects switched on and off, respectively. The shaded region reflects uncertainties in the CGC predictions due to uncertainties in the knowledge of the saturation scale, $Q_{s}$. This comparison nicely demonstrates the discrimination power of these measurements. In fact, already with a fraction of the statistics used here one will be able to exclude one of the scenarios conclusively.

The left panel of fig. 50 depicts the predicted suppression through $J_{e \mathrm{Au}}$, the relative yield of correlated back-toback hadron pairs in $e+\mathrm{Au}$ collisions compared to $e+p$ collisions scaled down by $A^{1 / 3}$ (the number of nucleons at a fixed impact parameter)

$$
J_{e A}=\frac{1}{A^{1 / 3}} \frac{\sigma_{e A}^{\text {pair }} / \sigma_{e A}}{\sigma_{e p}^{\text {pair }} / \sigma_{e p}} .
$$

Here, $\sigma$ and $\sigma^{\text {pair }}$ are the total inelastic and the di-hadron pair production cross-sections (or normalized yields). The absence of collective nuclear effects in the pair production cross section, $\sigma_{e A}^{\text {pair }}$, would correspond to $J_{e A}=1$ (see footnote ${ }^{11}$ ), while $J_{e A}<1$ would signify suppression of di-hadron correlations. In the left panel of fig. 50, $J_{e A u}$ is plotted as a function of $x_{A}^{f r a g}$, which is an approximation of the longitudinal momentum fraction of the probed gluon $x_{g}$ derived from the kinematics of the measured hadrons assuming they carry the full parton energy. Compared to the measurement shown in the right panel

11 Without collective nuclear effects the hadron pairs are produced in independent electron-nucleon scatterings, such that $\sigma_{e A}^{p a i r}=A \sigma_{e p}^{p a i r}$. The cross-section for inelastic $e+\mathrm{A}$ collisions, $\sigma_{e A}$, is related to the probability for the incoming electron (or, more precisely, $\gamma^{*} \rightarrow q \bar{q}$ ) to get the first inelastic collision, which usually takes place on the nuclear surface: hence $\sigma_{e A}=A^{2 / 3} \sigma_{e p}$. Combining these results we get $J_{e A}=1[200]$. 
of fig. 49 this study requires the additional $e+p$ baseline measurement but has the advantage of several experimental uncertainties canceling out. It is instructive to compare this plot with the equivalent measurement in $d+\mathrm{Au}$ collisions at RHIC [195] shown in the right panel of fig. 50. In $d+\mathrm{Au}$ collisions $J_{d \mathrm{Au}}$ is defined by analogy to eq. (28) with $A^{1 / 3}$ in the denominator replaced by the number of the binary nucleon-nucleon collisions $N_{\text {coll }}$ at a fixed impact parameter [195]. In both colliding systems, $e+\mathrm{Au}$ and $d+\mathrm{Au}$, the exact momentum fraction of the gluon $x_{g}$ cannot be directly measured experimentally and has to be ultimately modeled. However, these calculations are much better constrained in DIS where the key kinematic variables $x$ and $Q^{2}$ are known precisely, allowing for tighter constraints on $x_{g}$. The two curves in the right panel of fig. 50 represent the CGC calculations from [192-194] but without the Sudakov form-factor and appear to describe the data rather well. This example nicely demonstrates on the one hand the correspondence between the physics in $p(d)+\mathrm{A}$ and $e+\mathrm{A}$ collisions but on the other hand the lack of precise control in $p+\mathrm{A}$ that is essential for precision studies of saturation phenomena.

\section{Measurements of diffractive events}

Diffractive interactions result when the electron probe in DIS interacts with a proton or nucleus by exchanging several partons with zero net color. This exchange, which in QCD may be visualized as a colorless combination of two or more gluons, is commonly referred to as the "Pomeron" (see Sidebar V).

The HERA physics program of $e+p$ collisions surprisingly showed a large fraction of diffractive events contributing about $15 \%$ to the total DIS cross-section [189]. One of the key signatures of these events is an intact proton traveling at near-to beam energies, together with a gap in rapidity before some final-state particles are produced at mid-rapidity (i.e., at $90^{\circ}$ angle to the beam axis). While linear pQCD is able to describe some aspects of diffraction, it fails to describe other major features without introducing new types of structure functions, the diffractive structure functions (see Sidebar V), which describe the rapidity gap. A striking example is the fact that the ratio of the diffractive to the total cross-section is constant with energy, an observation not easily reconciled in a conventional pQCD scenario without introducing the diffractive structure functions [189]. As may therefore be anticipated, and as we have argued above, the strongest hints for a manifestations of new, non-linear effects in $e+\mathrm{A}$ collisions are likely to come from diffractive measurements.

What makes the diffractive processes so interesting is that they are most sensitive to the underlying gluon distribution, and that they are the only known class of events that allows us to gain insight into the spatial distribution of gluons in nuclei. The reason for this sensitivity is that the diffractive structure functions depend, in a wide kinematic range, quadratically on the gluon momentum distribution and not linearly as in DIS. However, while the physics goals are golden, the technical challenges are formidable but not insurmountable, and require careful planning of the detector and interaction region. Diffractive events are characterized by a rapidity gap, i.e. an angular region in the direction of the scattered proton or nucleus without particle flow. Detecting events with rapidity gaps requires a largely hermetic detector.

As discussed earlier (see sect. 3.2.1) we distinguish two kinds of diffractive events: coherent (nucleus stays intact) and incoherent (nucleus excites and breaks up). Both contain a rich set of information. Coherent diffraction is sensitive to the space-time distribution of the partons in the nucleus, while incoherent diffraction (dominating at larger $t$ and thus small impact parameter $b_{T}$ ) is most sensitive to high parton densities where saturation effects are stronger. In $e+p$ collisions, the scattered intact protons can be detected in a forward spectrometer placed many meters down the beam line. This is not possible for nuclei which, due to their large mass, stay too close to the ion beam. However, studies showed that the nuclear breakup in incoherent diffraction can be detected with close to $100 \%$ efficiency by measuring the emitted neutrons in a zerodegree calorimeter placed after the first dipole magnet that bends the hadron beam. This tagging scheme could be further improved by using a forward spectrometer to detect charged nuclear fragments. A rapidity gap and the absence of any break-up fragments was found sufficient to identify coherent events with very high efficiency.

In the following, we present several measurements focusing on the discrimination power between non-linear saturation models and a prediction from conventional linear QCD DGLAP evolution. Saturation models incorporate the effects of linear small- $x$ evolution for $Q>Q_{s}$ and saturation non-linear evolution effects for $Q<Q_{s}$.

Ratio of diffractive and total cross-sections. Figure 51 depicts predictions for one of the simplest inclusive measurements that can be performed with diffractive events: the measurement of the ratio of the coherent diffractive cross-section over the total cross-section in $e+p$ and $e+\mathrm{A}$ collisions is shown at the top of each panel. It is plotted here as a function of the diffractive mass of the produced final state particles, $M_{X}^{2}$ (see Sidebar V), for $x=10^{-3}$ and $Q^{2}=1$ and $5 \mathrm{GeV}^{2}$. For fixed $Q^{2}$ and $x, M_{X}^{2}$ can also be expressed in terms of the fraction of the momentum of the pomeron that is carried by the struck quark within the proton or nucleus, $\beta$, shown along the alternative abscissa on the top of each plot where $\beta \approx \frac{Q^{2}}{Q^{2}+M_{X}^{2}}$, corresponding to a rapidity gap $\approx \ln (\beta / x)$. The red curves represent the predictions of the saturation model [201-204] based on Model-I of sect. 3.2.1 combined with the theoretical developments of $[168,205,206]$, while the blue curves and bands in the right panel represent the leading-twist shadowing (LTS) model [207,208]. The bottom part of each panel depicts the double ratio $\left[\left(\mathrm{d} \sigma_{\text {diff }} / \mathrm{d} M_{X}^{2}\right) / \sigma_{\text {tot }}\right]_{e A} /\left[\left(\mathrm{d} \sigma_{\text {diff }} / \mathrm{d} M_{X}^{2}\right) / \sigma_{\text {tot }}\right]_{e p}$, illustrating the fact that the fraction of diffractive over total cross section is expected to be higher in $e+\mathrm{A}$ than in $e+p$ in the saturation framework. The curves in fig. 51 are plotted for the range of $x$ and $Q^{2}$ values which will be accessible already at low to moderate EIC energies. The $e+p$ curves in both approaches are in a reasonable agreement with the 
$\beta$

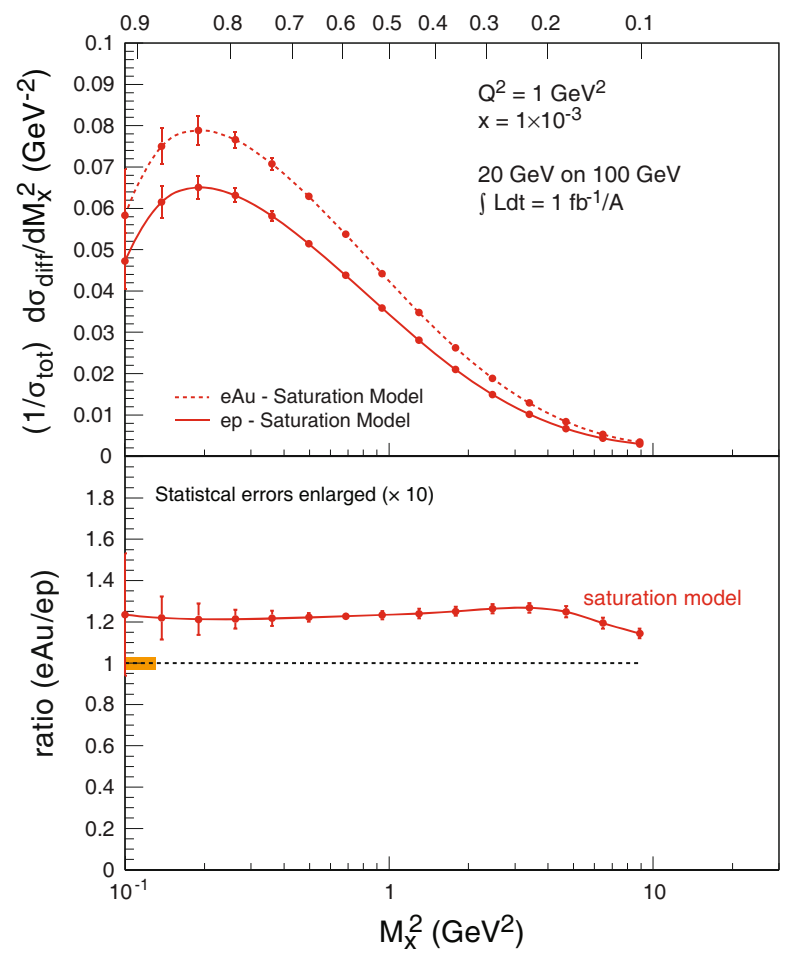

$\beta$

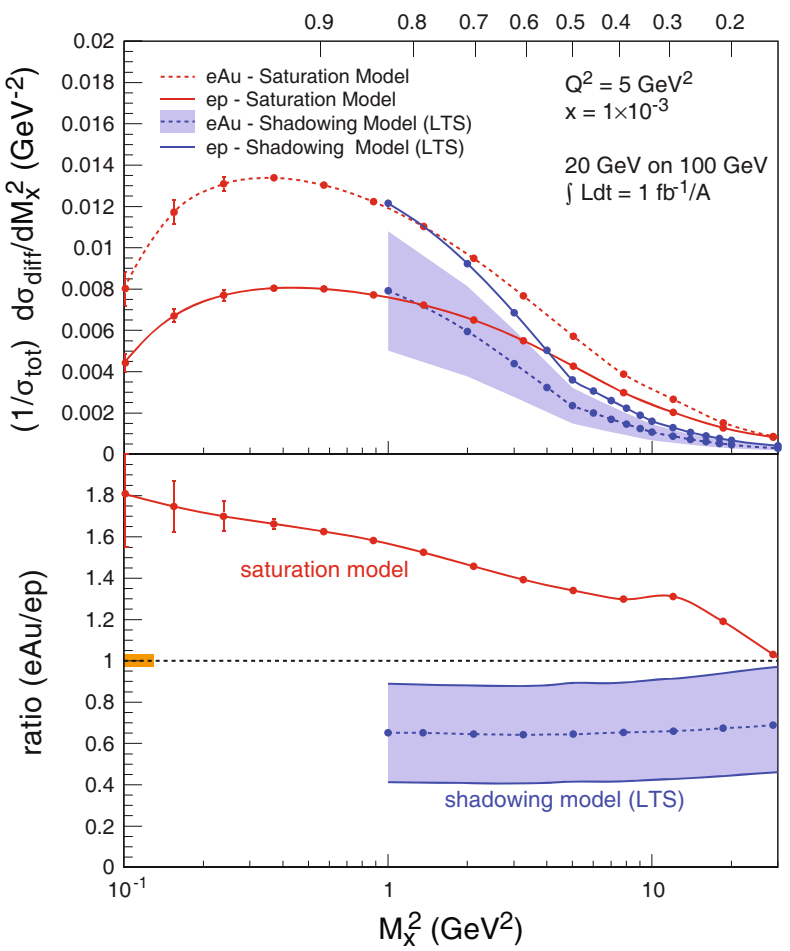

Fig. 51. Top of each panel: the ratio of diffractive over total cross-sections, plotted as a function of the invariant mass of the produced particles, $M_{X}^{2}$. The bottom of each panel contains the double ratio $\left[\left(\mathrm{d} \sigma_{\text {diff }} / \mathrm{d} M_{X}^{2}\right) / \sigma_{\text {tot }}\right]_{e A} /\left[\left(\mathrm{d} \sigma_{\mathrm{diff}} / \mathrm{d} M_{X}^{2}\right) / \sigma_{\text {tot }}\right]_{e p}$ plotted as a function of $M_{X}^{2}$ for the same kinematics as used at the top of each panel. The statistical error bars for the integrated luminosity of $1 \mathrm{fb}^{-1} / \mathrm{A}$ are too small to depict and are enlarged by a factor 10 . The non-monotonicity of the saturation curve in the lower panels is due to crossing the $c \bar{c}$ threshold; this threshold is not included in the LTS prediction.

available HERA data [2, 201]. The statistical error bars, shown in the bottom parts of the panels in fig. 51 are very small, and had to be scaled up by a factor of 10 to become visible. We conclude that the errors of the actual measurement would be dominated by the systematic uncertainties dependent on the quality of the detector and on the luminosity measurements. The size of the error bars shows that the two scenarios can be clearly distinguished over a wide $x$ and $Q^{2}$ range, allowing for a clear early measurement aimed at finding evidence of parton saturation.

Note that in the saturation predictions plotted in fig. 51, the nuclear effects, responsible for the difference between the $e+\mathrm{Au}$ and $e+p$ curves, are stronger at large $Q^{2}$ : the effect of saturation is to weaken the $A$-dependence in the $\sigma_{\text {diff }} / \sigma_{\text {tot }}$ ratio at low $Q^{2}$. Also, in agreement with the expectation that diffraction would be a large fraction of the total cross-section with the onset of the black disk limit (see eq. $(27)$ ), the ratio $\left(\mathrm{d} \sigma_{\text {diff }} / \mathrm{d} M_{X}^{2}\right) / \sigma_{\text {tot }}$ plotted in fig. 51 both for $e+p$ and $e+$ Au grows with decreasing $Q^{2}$, getting larger as one enters the saturation region.

The ratio of the diffractive to total cross-section, $\sigma_{\text {diff }} / \sigma_{\text {tot }}$, evaluated in a CGC model $[203,204]$, is plotted in fig. 52 as a function of the center-of-mass energy of the virtual photon-proton (nucleus) system $W$ (see Sidebar I) for $e+p$ and $e+\mathrm{A}$ scattering with $Q^{2}=1$ and $10 \mathrm{GeV}^{2}$. Again the diffractive to total cross section ratio is higher in $e+\mathrm{A}$ than in $e+p$. Intriguingly, the ratio be-

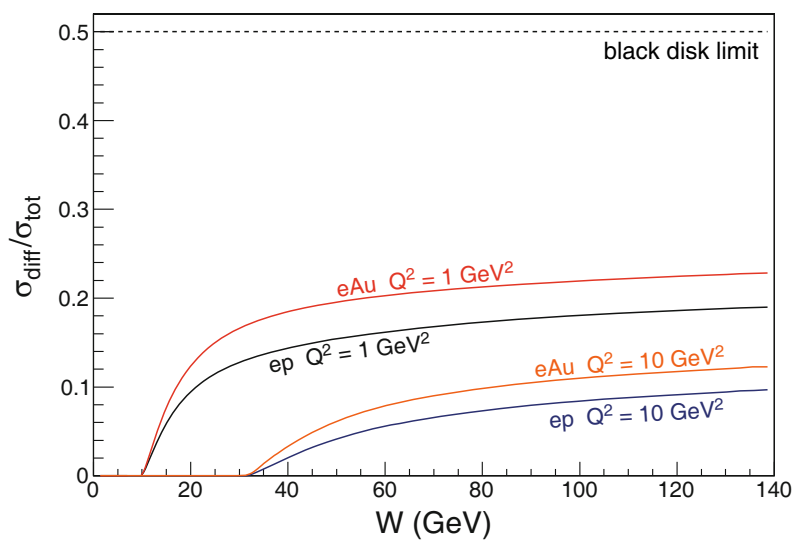

Fig. 52. The ratio of the diffractive to total cross sections as a function of the center-of-mass energy of the virtual photonproton (nucleus) system $W$.

comes almost independent of energy $W$ for high enough $W$ : such behavior was already observed in $e+p$ scattering at HERA [209]. (The ratio in fig. 52 is always much lower than its black-disk value of $1 / 2$ due to the fact that even at very high energies saturation is not yet reached at the edges of the proton or nucleus.) This energy-independence has a particularly simple explanation in the saturation framework as being due to the energy-dependent infrared 

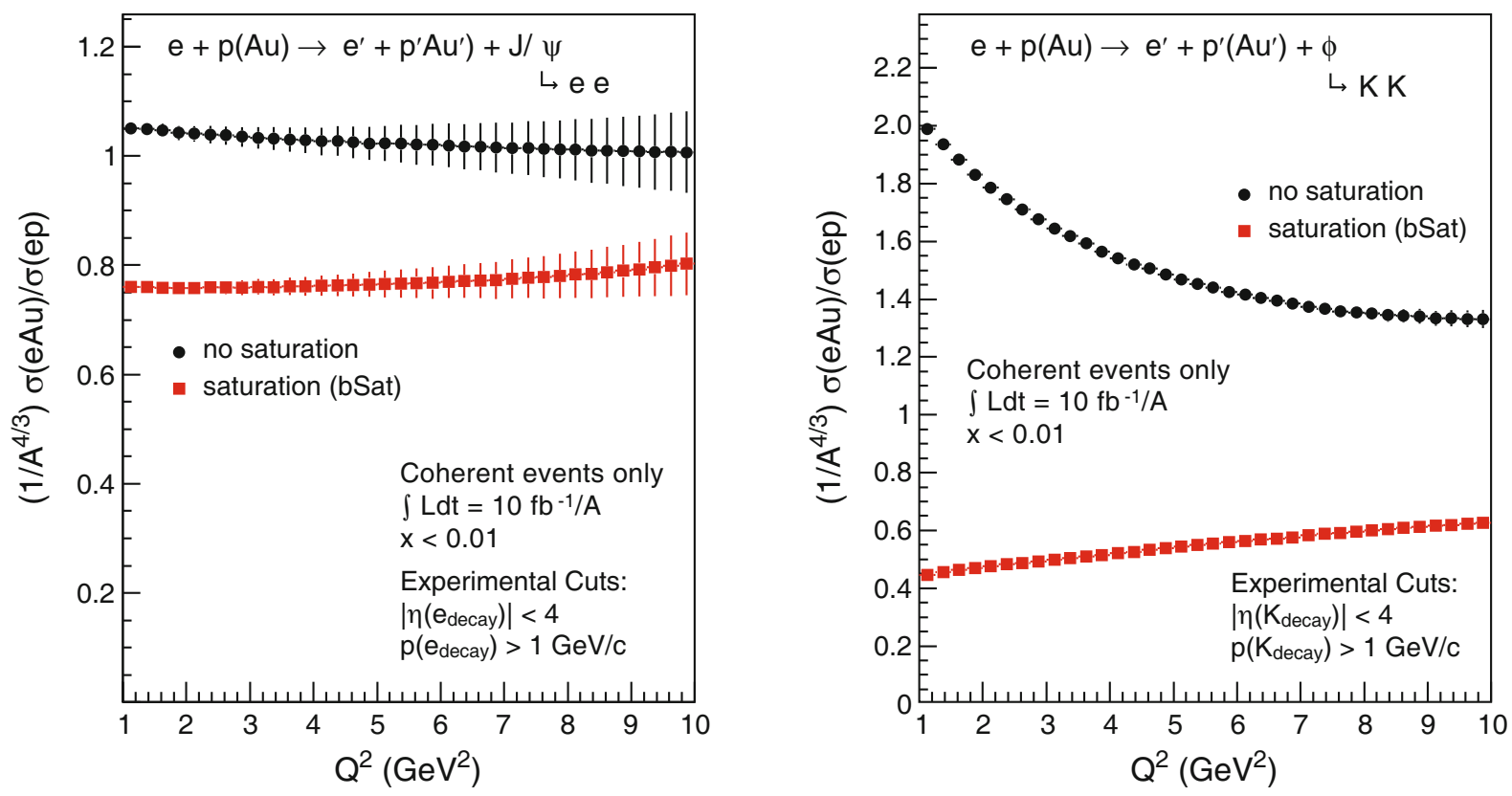

Fig. 53. Ratios of the cross-sections for exclusive $J / \psi$ (left panel) and $\phi$ (right panel) meson production in coherent diffractive $e+\mathrm{A}$ and $e+p$ collisions as a function of $Q^{2}$ at an EIC with $20 \mathrm{GeV}$ on $100 \mathrm{GeV}$ beam energies. Prediction for saturation and non-saturation models are presented. The ratios are scaled by $1 / A^{4 / 3}$.

cutoff $Q_{s}$ [206], suggesting that saturation effects may possibly have been observed at HERA: it would be important to make sure that this energy-independence of the diffractive to total cross section ratio remains to be the case at EIC.

Diffractive vector meson production. The production of vector mesons in diffractive processes, $e+\mathrm{A} \rightarrow e^{\prime}+\mathrm{A}^{\prime}+V$ where $V=J / \psi, \phi, \rho$, or $\gamma$, is a unique process, for it allows the measurement of the momentum transfer, $t$, at the hadronic vertex even in $e+$ A collisions where the 4momentum of the outgoing nuclei cannot be measured. Since only one new final state particle is generated, the process is experimentally clean and can be unambiguously identified by the presence of a rapidity gap. The study of various vector mesons in the final state allows a systematic exploration of the saturation regime [188]. The $J / \psi$ is the vector meson least sensitive to saturation effects due to the small size of its wave-function. Larger mesons such as $\phi$ or $\rho$ are considerably more sensitive to saturation effects [210].

The two panels in fig. 53 show the ratios $\left[\mathrm{d} \sigma(e \mathrm{Au}) / \mathrm{d} Q^{2}\right] /\left[\mathrm{d} \sigma(e p) / \mathrm{d} Q^{2}\right]$ (scaled down by $A^{4 / 3}$ ) of the cross-sections $\sigma(e+\mathrm{Au})$ and $\sigma(e+p)$ for exclusive $J / \psi$ (left panel) and $\phi$ (right panel) production in coherent diffractive events for $e+\mathrm{Au}$ and $e+p$ collisions respectively. The ratios are plotted as functions of $Q^{2}$ for saturation and non-saturation models. The parameters of both models were tuned to describe the $e+p$ HERA data $[174,210]$. All curves were generated with the Sartre event generator [211], an $e+\mathrm{A}$ event generator specialized for diffractive exclusive vector meson production based on the bSat [210] dipole model. We limit the calculation to $1<Q^{2}<10 \mathrm{GeV}^{2}$ and $x<0.01$ to stay within the validity range of saturation and non-saturation models. The produced events were passed through an experimental filter and scaled to reflect an integrated luminosity of $10 \mathrm{fb}^{-1} / \mathrm{A}$. The basic experimental cuts are listed in the legends of the panels in fig. 53. As expected, the difference between the saturation and non-saturation curves is small for the smaller-sized $J / \psi(<20 \%)$, which is less sensitive to saturation effects, but is substantial for the larger $\phi$, which is more sensitive to the saturation region. In both cases, the difference is larger than the statistical errors. In fact, the small errors for diffractive $\phi$ production indicate that this measurement can already provide substantial insight into the saturation mechanism after a few weeks of EIC running. Although this measurement could be already feasible at an EIC with low collision energies, the saturation effects would be less pronounced due to the larger values of $x$. For large $Q^{2}$, the two ratios asymptotically approach unity.

As explained earlier in sect. 3.2.1, coherent diffractive events allow one to learn about the shape and the degree of "blackness" of the black disk: this enables one to study the spatial distribution of gluons in the nucleus. Exclusive vector meson production in diffractive $e+\mathrm{A}$ collisions is the cleanest such process, due to the low number of particles in the final state. This would not only provide us with further insight into saturation physics but also constitute a highly important contribution to heavy-ion physics by providing a quantitative understanding of the initial conditions of a heavy-ion collision as described in sect. 3.4.2. It might even shed some light on the role of glue and thus QCD in the nuclear structure of light nuclei (see sect. 3.3). As described above, in diffractive DIS, the virtual photon interacts with the nucleus via a color-neutral exchange, 

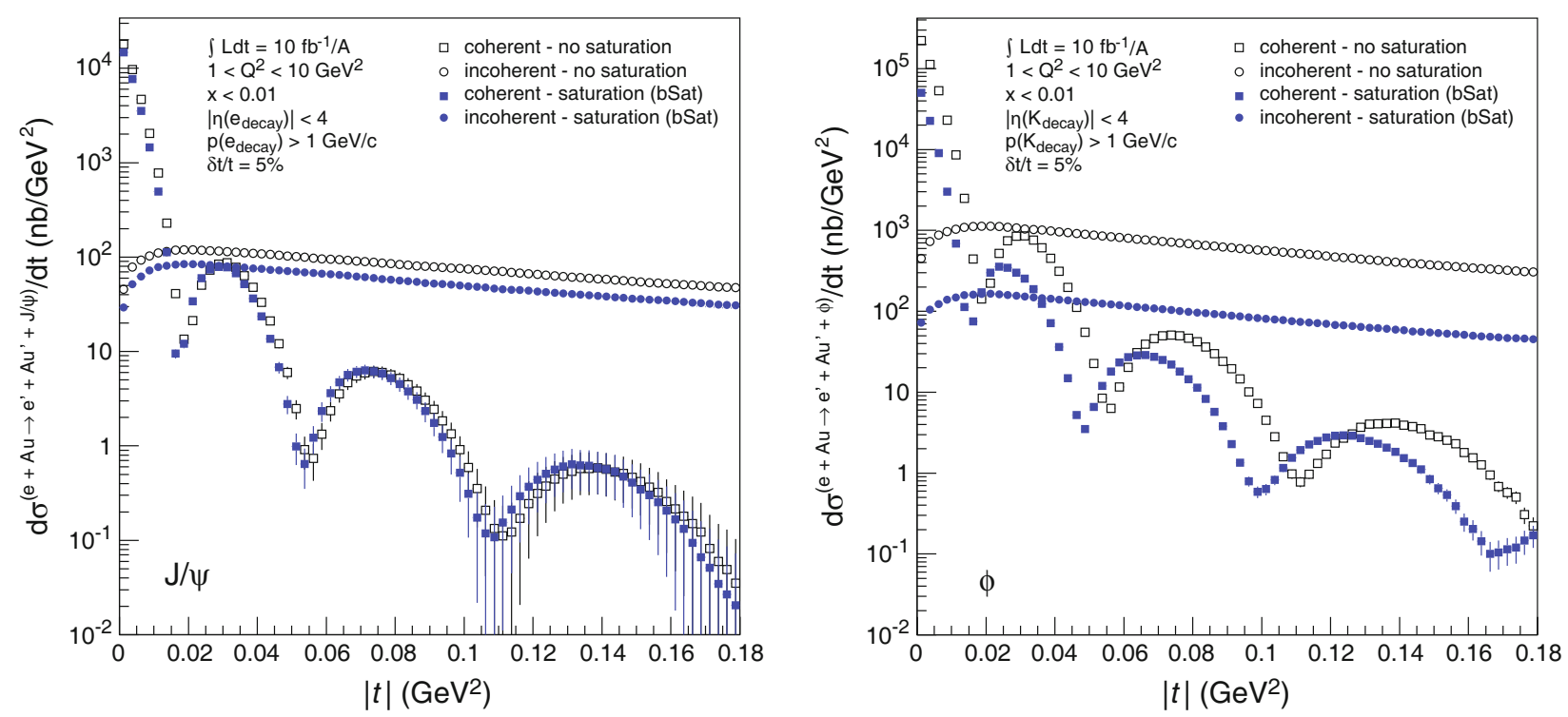

Fig. 54. $\mathrm{d} \sigma / \mathrm{d} t$ distributions for exclusive $J / \psi$ (left) and $\phi$ (right) production in coherent and incoherent events in diffractive $e+\mathrm{Au}$ collisions. Predictions from saturation and non-saturation models are shown.

which is dominated by two gluons at the lowest order. It is precisely this two-gluon exchange which yields a diffractive measurement of the gluon density in a nucleus.

Experimentally the key to the spatial gluon distribution is the measurement of the $\mathrm{d} \sigma / \mathrm{d} t$ distribution. As follows from the optical analogy presented in sect. 3.2.1, the Fourier transform of (the square root of) this distribution is the source distribution of the object probed, i.e., the dipole scattering amplitude $N\left(x, r_{T}, b_{T}\right)$ on the nucleus with $r_{T}^{2} \sim 1 /\left(Q^{2}+M_{V}^{2}\right)$, where $M_{V}$ is the mass of the vector meson [188] (see also Sidebar IV). Note that related studies can be conducted in ultra-peripheral collisions of nuclei, albeit with a limited kinematic reach. This is discussed in sect. 3.4.2.

Figure 54 shows the $\mathrm{d} \sigma / \mathrm{d} t$ distribution for $J / \psi$ on the left and $\phi$ mesons on the right. The coherent distribution depends on the shape of the source while the incoherent distribution provides valuable information on the fluctuations or "lumpiness" of the source [201]. As discussed above, we are able to distinguish both by detecting the neutrons emitted by the nuclear breakup in the incoherent case. Again, we compare to predictions of saturation and non-saturation models. Just as for the previous figures, the curves were generated with the Sartre event generator and had to pass through an experimental filter. The experimental cuts are listed in the figures.

As the $J / \psi$ is smaller than the $\phi$, one sees little difference between the saturation and no-saturation scenarios for exclusive $J / \psi$ production but a pronounced effect for the $\phi$, as expected. For the former, the statistical errors after the 3rd minimum become excessively large requiring substantially more than the simulated integrated luminosity of $10 \mathrm{fb}^{-1} / \mathrm{A}$. The situation is more favorable for the $\phi$, where enough statistics up to the 4th minimum are available. The $\rho$ meson has even higher rates and is also quite sensitive to saturation effects. However, it suffers currently from large theoretical uncertainties in the knowledge of its wave-function, making calculations less reliable.

The coherent distributions in fig. 54 can be used to obtain information about the gluon distribution in impact parameter space $F(b)$ through a two-dimensional Fourier transform of the square root of the coherent elastic cross section $[188,203]$

$$
F(b)=\int_{0}^{\infty} \frac{\mathrm{d} q q}{2 \pi} J_{0}(q b) \sqrt{\frac{\mathrm{d} \sigma_{\text {coherent }}}{\mathrm{d} t}}
$$

with $t=-q^{2}$. In fig. 55 we show the resulting Fourier transforms of the coherent points in fig. 54, using the range $-t<0.36 \mathrm{GeV}^{2}$ which is achievable at the EIC given enough statistics. As a reference, we show (dotted line) the original input source distribution used in the generator, which is the Woods-Saxon function integrated over the longitudinal direction. The obtained distributions have been normalized to unity. The uncertainties due to the statistical error are negligible, and are barely visible in fig. 55 . Strictly-speaking, the integral over $t$ in the Fourier transformation should be performed up to $|t| \rightarrow \infty$. We studied the effects of using the finite $t$-range in the Fourier transform by varying the upper integration limit and found fast convergence towards the input Woods-Saxon distribution already for the upper limit of $|t| \sim 0.1 \mathrm{GeV}^{2}$.

The non-saturation curves for $\phi$ and $J / \psi$-meson production reproduce the shape of the input distribution perfectly. For the saturation model, the shape of the $J / \psi$ curve also reproduces the input distribution, while the $\phi$ curve does not. As explained above, this is expected, as the size of the $J / \psi$ meson is much smaller than that for $\phi$, making the latter more susceptible to non-linear effects as already observed in figs. 53 and 54 . We conclude that the $J / \psi$ meson is better suited for probing the transverse structure of the nucleus. However, by measuring $F(b)$ with 

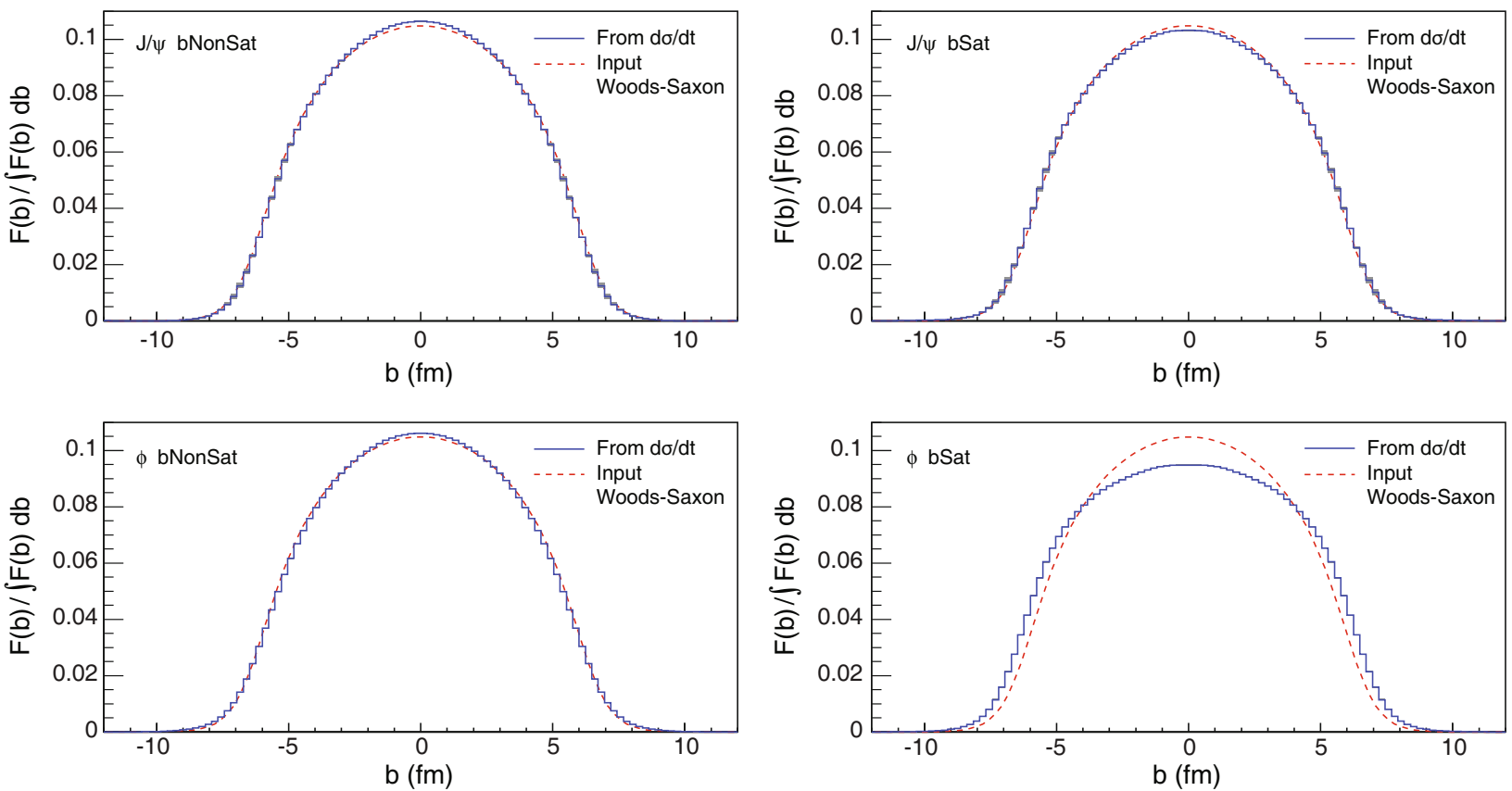

Fig. 55. The Fourier transforms obtained in [203] from the distributions in fig. 54 for $J / \psi$-mesons in the upper row and $\phi$ mesons in the lower row. The results from both saturation (right) and non-saturation (left) models are shown. The used input Woods-Saxon distribution is shown as a reference in all four plots.

both $J / \psi$ and $\phi$ mesons, one can obtain valuable information on how sensitive the measurement is to non-linear effects. Thus, both measurements are important and complementary to each other. The results in fig. 55 provide a strong indication that EIC will be able to obtain the nuclear spatial gluon distribution from the measured coherent $t$-spectrum from exclusive $J / \psi$ and $\phi$ production in $e+\mathrm{A}$, in a model-independent fashion.

\subsection{Quarks and gluons in the nucleus ${ }^{12}$}

Here we present a few key measurements that will allow us to answer the fundamental questions from the beginning of this section and to explore the properties of quarks and gluons and their interactions in a nuclear environment. In table 5, we list the key measurements to be carried out at an EIC. The measurement of nuclear structure functions with various ion beams at intermediate- $x$ will enable the first glimpses of collective nuclear effects at the partonic level and the onset of the breakdown of DGLAP evolution. The semi-inclusive production of energetic hadrons will probe nuclear matter's response to a fast moving color charge as well as the mass of the particle carrying the charge. The multiple scattering of the fast moving color charge off the color field inside the nucleus could modify the distribution of produced hadrons. The transversemomentum broadening of the produced hadrons in $e+\mathrm{A}$ collisions provides a sensitive probe to the characteristic time scale (or distance) of color neutralization, as well

\footnotetext{
12 Conveners: William Brooks and Jian-Wei Qiu.
}

as the response of the nuclear medium to a fast moving color charge. It thus allows access to the transport coefficients of the nuclear system and to medium induced energy loss mechanisms. With the well-determined leptonic and hadronic scattering planes, and the azimuthal angle $\phi$ between the planes in semi-inclusive DIS, on an event-by-event basis, the nuclear modification to the angular $\phi$ modulation of the produced hadrons could be a sensitive probe of the fluctuation of spatial distributions of quarks and gluons inside a large nucleus [212], which is very important for understanding the initial condition of relativistic heavy ion collisions.

\subsubsection{Distributions of quarks and gluons in a nucleus}

The momentum distribution of quarks and gluons inside a fast-moving proton was best measured by lepton DIS on a proton beam at HERA. Although the scattering could take place between the lepton and a single-quark (or gluon) state as well as a multiple quark-gluon state of the proton, the large momentum transfer of the scattering, $Q$, localizes the scattering, suppresses the contribution from multiple scattering, and allows us to express the complex DIS cross-sections in terms of a set of momentum distributions of quarks and gluons. These are probability density distributions to find a parton (quark, anti-quark or gluon) to carry the momentum fraction $x$ of a fastmoving hadron. Actually, it is a triumph of QCD that one set of universal parton distributions, extracted from HERA data, plus calculable scatterings between quarks and gluons, can successfully interpret all existing data of 
Table 5. Key measurements in $e+\mathrm{A}$ collisions at an EIC to explore the dynamics of quarks and gluons in a nucleus in the non-saturation regime.

\begin{tabular}{|c|c|c|}
\hline Deliverables & Observables & What we learn \\
\hline \hline $\begin{array}{c}\text { Collective } \\
\text { nuclear effects } \\
\text { at intermediate } x\end{array}$ & $\begin{array}{c}\text { Ratios } R_{2} \\
\text { from inclusive DIS }\end{array}$ & $\begin{array}{c}Q^{2} \text { evolution: onset of DGLAP violation, beyond DGLAP } \\
A \text {-dependence of shadowing and antishadowing } \\
\text { Initial conditions for small- } x \text { evolution }\end{array}$ \\
\hline $\begin{array}{c}\text { Transport } \\
\text { coefficients in } \\
\text { nuclear matter }\end{array}$ & $\begin{array}{c}\text { Production of light } \\
\text { and heavy hadrons, }\end{array}$ & $\begin{array}{c}\text { Color neutralization: mass dependence of hadronization } \\
\text { Multiple scattering and mass dependence of energy loss } \\
\text { and in SIDIS }\end{array}$ \\
\hline $\begin{array}{c}\text { Nuclear density } \\
\text { and its fluctuation }\end{array}$ & $\begin{array}{c}\text { Hadron production } \\
\text { in SIDIS }\end{array}$ & $\begin{array}{c}\text { Transverse momentum broadening of produced hadrons } \\
\text { Azimuthal } \phi \text {-modulation of produced hadrons }\end{array}$ \\
\hline
\end{tabular}

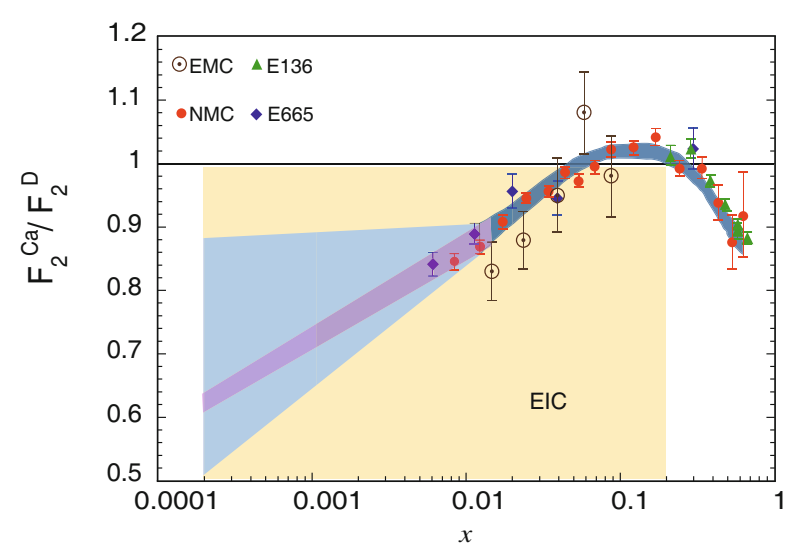

Fig. 56. The ratio of nuclear over nucleon $F_{2}$ structure function, $R_{2}$, as a function of Bjorken $x$, with data from existing fixed target DIS experiments at $Q^{2}>1 \mathrm{GeV}^{2}$, along with the QCD global fit from EPS09 [176]. Also shown is the expected kinematic coverage of the inclusive measurements at the EIC. The purple error band is the expected systematic uncertainty at the EIC assuming a $\pm 2 \%$ (a total of $4 \%$ ) systematic error, while the statistical uncertainty is expected to be much smaller.

high-energy proton collisions with a momentum transfer larger than $2 \mathrm{GeV}$ (corresponding to hard scatterings taking place at a distance less than one tenth of a femtometer).

Are the quarks and gluons in a nucleus confined within the individual nucleons? Or does the nuclear environment significantly affect their distributions? The EMC experiment at CERN [213] and experiments in the following two decades clearly revealed that the momentum distribution of quarks in a fast-moving nucleus is not a simple superposition of their distributions within nucleons. Instead, the measured ratio of nuclear over nucleon structure functions, as defined in eq. (23), follows a non-trivial function of Bjorken $x$, significantly different from unity, and shows the suppression as $x$ decreases, as shown in fig. 56 . The observed suppression at $x \sim 0.01$, which is often referred to as the phenomenon of nuclear shadowing, is much stronger than what the Fermi motion of nucleons inside a nucleus could account for. This discovery sparked a worldwide ef- fort to study the properties of quarks and gluons and their dynamics in the nuclear environment both experimentally and theoretically.

Using the same very successful QCD formulation at the leading power in $Q$ for proton scattering, and using the DGLAP evolution for the scale dependence of parton momentum distributions, several QCD global analyses have been able to fit the observed non-trivial nuclear dependence of existing data, attributing all observed nuclear dependences - including its $x$-dependence and nuclear atomic weight $A$-dependence - to a set of nucleusdependent quark and gluon distributions at an input scale $Q_{0} \gtrsim 1 \mathrm{GeV}[176,178,179]$. As an example, the fitting result of Eskola et al. is plotted along with the data on the ratio of the $F_{2}$ structure function of calcium divided by that of deuterium in fig. 56, where the dark blue band indicates the uncertainty of the EPS09 fit [176]. The success of the QCD global analyses clearly indicates that the response of the nuclear cross-section to the variation of the probing momentum scale $Q \gtrsim Q_{0}$ is insensitive to the nuclear structure, since the DGLAP evolution itself does not introduce any nuclear dependence. However, it does not answer the fundamental questions: Why are the parton distributions in a nucleus so different from those in a free nucleon at the probing scale $Q_{0}$ ? How do the nuclear structure and QCD dynamics determine the distributions of quarks and gluons in a nucleus?

The nucleus is a "molecule" in QCD, made of nucleons - which, in turn, are bound states of quarks and gluons. Unlike the molecule in QED, nucleons in the nucleus are packed next to each other, and there are many soft gluons inside nucleons when probed at small $x$. The DIS probe has a high resolution in transverse size $\sim 1 / Q$. But its resolution in the longitudinal direction, which is proportional to $1 / x p \sim 1 / Q$, is not necessarily sharp in comparison with the Lorentz contracted size of a light-speed nucleus, $\sim 2 R_{A}(m / p)$, with nuclear radius $R_{A} \propto A^{1 / 3}$ and the Lorentz contraction factor $m / p$ and nucleon mass $m$. That is, when $1 / x p>2 R_{A}(m / p)$, or at a small $x \sim 1 / 2 m R_{A} \sim 0.01$, the DIS probe could interact coherently with quarks and gluons of all nucleons at the same impact parameter of the largest nucleus moving nearly at the speed of light, $p \gg m$. The destructive interference of the coherent multiple scattering could lead to a 
reduction of the DIS cross-section $[152,208]$. Such coherent multi-parton interactions at small $x$ could take place non-perturbatively to generate a nuclear dependence of the parton distributions at the input scale $Q_{0}$, including shadowing [208] and anti-shadowing [214], which could be systematically extracted by using the DGLAP-based leading power QCD formalism. In addition, coherent multiple scattering could also take place at a perturbative scale $Q>Q_{0}$, and its contribution to the inclusive DIS crosssection could be systematically investigated in QCD in terms of corrections to the DGLAP-based QCD formulation $[215,216]$. Although such corrections are suppressed by the small perturbative probing size, they can be enhanced by the number of nucleons at the same impact parameter in a nucleus and large number of soft gluons in nucleons. Coherent multiple scattering naturally leads to the observed phenomena of nuclear shadowing: more suppression when $x$ decreases, $Q$ decreases, and $A$ increases. But, none of these dependences could have been predicted by the very successful leading power DGLAP-based QCD formulation.

When the gluon density is so large at small $x$ and the coherent multi-parton interactions are so strong that their contributions are equally important as that from single-parton scattering, measurements of the DIS crosssection could probe a new QCD phenomenon - the saturation of gluons discussed in the last section. In this new regime, which is referred to as a Color Glass Condensate (CGC) $[157,160]$, the standard fixed order perturbative QCD approach to the coherent multiple scattering would be completely ineffective. The resummation of all powers of coherent multi-parton interactions or new effective field theory approaches are needed. The RHIC data $[195,196]$ on the correlation in deuteron-gold collisions indicate that the saturation phenomena might take place at $x \lesssim 0.001[195,196]$. Therefore, the region of $0.001<x<0.1$, at a sufficiently large probing scale $Q$, could be the most interesting place to see the transition of a large nucleus from a diluted partonic system - whose response to the resolution of the hard probe (the $Q^{2}$ dependence) follows linear DGLAP evolution - to matter composed of condensed and saturated gluons.

This very important transition region with Bjorken $x \in(0.001,0.1)$ could be best explored by the EIC, as shown in fig. 56. The EIC will not only explore this transition region, but will also have a wide overlap with regions that have been and will be measured by fixed target experiments, as indicated by the yellow box in fig. 56. At its full operation, the coverage of EIC in $x$ could be extended down to $10^{-4}$ while maintaining a sufficiently large $Q$. The EIC will have ideal kinematic coverage for the systematic study of QCD dynamics in this very rich transition region, as well as the new regime of saturated gluons.

If the nuclear effect on the DIS cross-section, as shown in fig. 56, is mainly due to the abundance of nucleons at the same impact parameter of the nucleus (proportional to $A^{1 / 3}$ ), while the elementary scattering is still relatively weak, one would expect the ratio of nuclear over nucleon structure functions to saturate when $x$ goes below 0.01 , or equivalently, the nuclear structure function to be pro- portional to the nucleon structure functions, as shown, for example, by the upper line of the blue area extrapolated from the current data in fig. 56. In this case, there is no saturation in nuclear structure functions since the proton structure function is not saturated at this intermediate- $x$ region, and the ratio could have a second drop at a smaller $x$ when nuclear structure functions enter the saturation region. On the other hand, if the soft gluons are a property of the whole nucleus and the coherence is strong, one would expect the ratio of the nuclear to nucleon structure function to fall continuously as $x$ decreases, as sketched by the lower line of the blue band, and eventually, reach a constant when both nuclear and nucleon structure functions are in the saturation region. From the size of the purple error band in fig. 56, which is the expected systematic uncertainty at the EIC (while the statistical uncertainty is expected to be much smaller), the EIC could easily distinguish these two extreme possibilities to explore the nature of sea quarks and soft gluons in a nuclear environment.

With the unprecedented energy and luminosity of lepton-nucleus collisions at the EIC, the precision measurements of the $Q$-dependence of the nuclear structure functions could extract nuclear gluon distributions at small $x$ that are effectively unknown now, and identify the momentum scale $Q_{0}$ below which the DGLAP-based QCD formulation fails, to discover the onset of the new regime of non-linear QCD dynamics. With its variety of nuclear species, and the precise measurements of the $x$ and $Q$-dependence in this transition region, the EIC is an ideal machine to explore the transition region and to provide immediate access to the first glimpses of collective nuclear effects caused by coherent multi-parton dynamics in QCD. Inclusive DIS measurements at the EIC provide an excellent and unique testing ground to study the transition to new and novel saturation physics.

\subsubsection{Propagation of a fast-moving color charge in QCD matter}

The discovery of the quark-gluon plasma (QGP) in the collision of two heavy ions at the Relativistic Heavy Ion Collider (RHIC) at Brookhaven National Laboratory made it possible to study in a laboratory the properties of quarkgluon matter at extremely high temperatures and densities, which were believed to exist only a few microseconds after the Big Bang. One key piece of evidence of the discovery was the strong suppression of fast moving hadrons produced in relativistic heavy-ion collisions [217], which is often referred to as jet quenching [218]. It was found that the production rate of the fast moving hadrons in a central gold-gold collision could be suppressed by as much as a factor of five compared to that of a proton-proton collision at the same energy, and the same phenomenon was confirmed by the heavy ion program at the LHC.

Fast moving hadrons at RHIC are dominantly produced by the fragmentation of colored fast moving quarks or gluons that are produced during hard collisions at short distances. Fragmentation (or in general, Hadronization) - the transition of a colored and energetic parton 

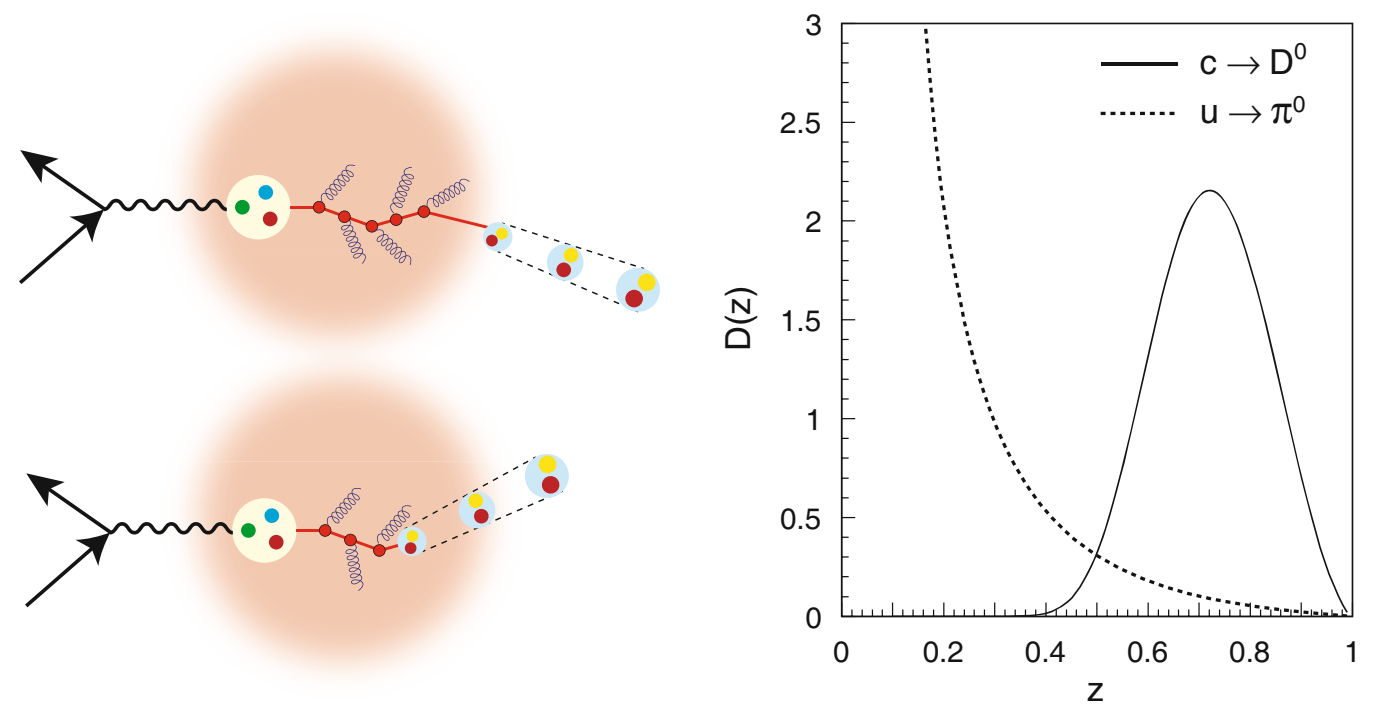

Fig. 57. Left: a cartoon for the interactions of the parton moving through cold nuclear matter when the produced hadron is formed outside (upper plot) and inside (lower plot) the nucleus. Right: fragmentation functions as a function of $z$ : from the charm quark to the $D^{0}$ meson (solid) [219] and from up quark to $\pi^{0}$ meson (dashed line) [42].

to a colorless hadron - is a rich and dynamical process in QCD quantified by the fragmentation function $D_{\text {parton } \rightarrow \text { hadron }}(z)$, with $z$ the momentum fraction of the fast moving parton to be carried by the produced hadron in the DGLAP based QCD formulation. Although QCD calculations are consistent with hadron production in high-energy collisions, knowledge about the dynamics of the hadronization process remains limited and strongly model dependent. It is clear that color is ultimately confined in these dynamical processes. The color of an energetic quark or a gluon produced in high-energy collisions has to be neutralized so that it can transmute itself into hadrons. Even the determination of a characteristic time scale for the color neutralization would shed some light on the properties of color confinement and help answer the question of what governs the transitions of quarks and gluons to hadrons.

The collision of a fast moving parton within the QGP could induce gluon radiation to reduce the parton's forward momentum and energy, while the parton-to-hadron fragmentation functions might not be affected since the energetic hadrons are likely to be formed outside the QGP due to time dilation, as indicated by the cartoon in fig. 57 (left, upper plot). The energy loss of the active parton would require a fragmentation function of a larger $z$ in order to produce a hadron with the same observed momentum as that produced in proton-proton collisions without energy loss [220]. However, it has been puzzling [221] that heavy-meson production in the same experiments at RHIC seems to be suppressed as much as the production of light mesons, although a heavy quark is much less likely to lose its energy via medium-induced radiation. It is critically important to have new and clean measurements, as well as independent tests of the energy-loss mechanisms, in or- der to have full confidence in jet quenching as a hard probe of QGP properties.

Semi-inclusive DIS in $e+\mathrm{A}$ collisions provides a known and stable nuclear medium ("cold QCD matter"), wellcontrolled kinematics of hard scattering, and a finalstate particle with well-known properties. The time for the produced quark (or gluon) to neutralize its color depends on its momentum and virtuality when it was produced. The process could take place entirely inside the nuclear medium, or outside the medium, or somewhere in-between, as indicated by the cartoon in fig. 57 (left) $[222,223]$. Cold QCD matter could be an excellent femtometer-scale detector of the hadronization process from its controllable interaction with the produced quark (or gluon). By facilitating studies on how struck partons propagate through cold nuclear matter and evolve into hadrons, as sketched in fig. 57 (left), the EIC would provide independent and complementary information essential for understanding the response of the nuclear medium to a colored fast-moving (heavy or light) quark. With its collider energies and thus the much larger range of $\nu$, the energy of the exchanged virtual photon, the EIC is unique for providing clean measurements of medium induced energy loss when the hadrons are formed outside the nuclear medium, while it is also capable of exploring the interplay between hadronization and medium-induced energy loss when the hadronization takes place inside the medium. In the latter case, color transparency may also play a role [222,224-226], and this is yet another important topic that can be independently explored with various techniques and measurements at the EIC [227].

The amount of the medium-induced energy loss and the functional form of the fragmentation functions should be the most important cause for the multiplicity ratio of 

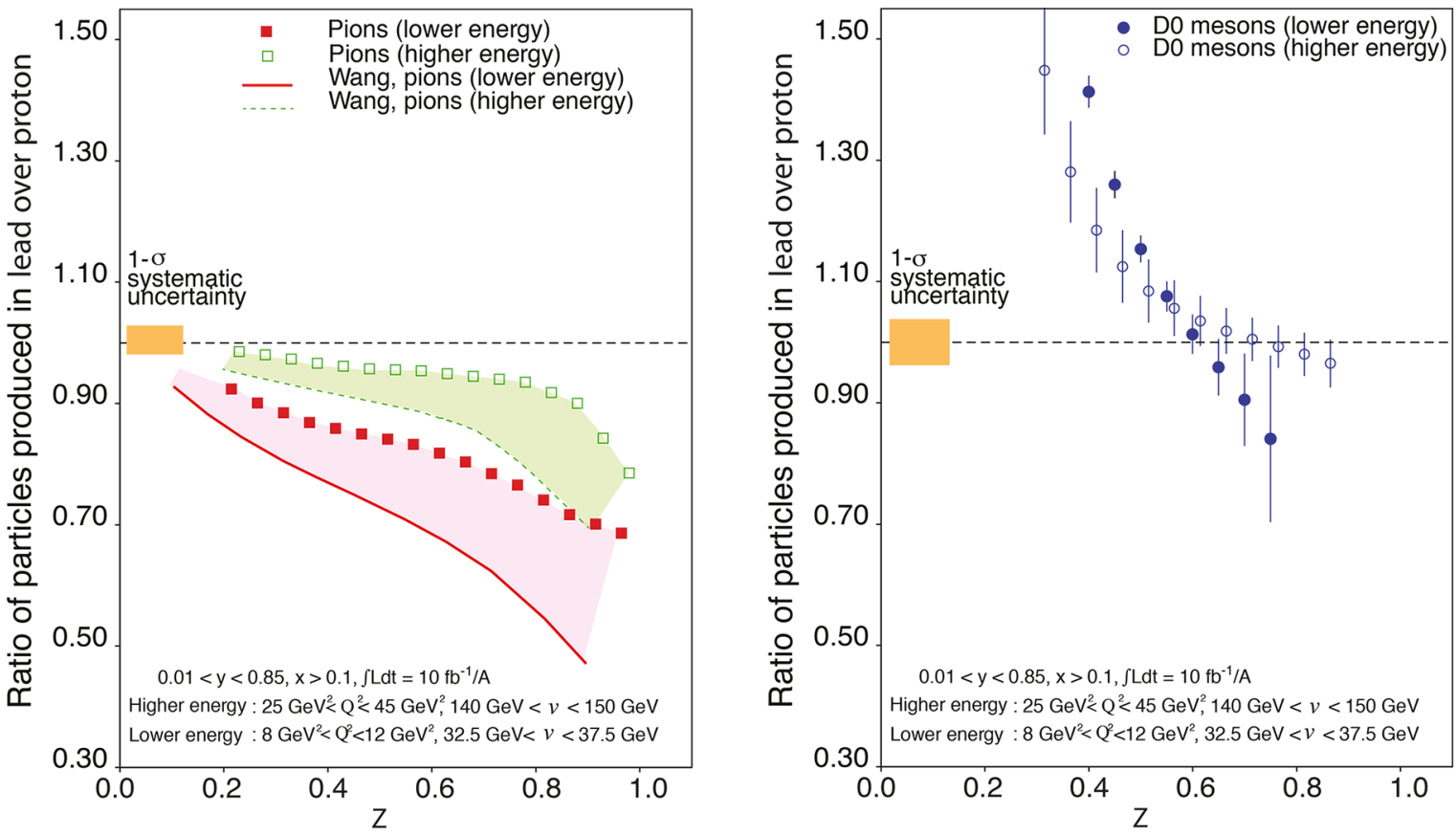

Fig. 58. The ratio of semi-inclusive cross-sections for producing a single pion (left) and a single $D^{0}$ (right) in electron-lead collisions to the same produced in electron-deuteron collisions as a function of $z$ at the EIC with two different photon energies $\nu=35 \mathrm{GeV}$ at $Q^{2}=10 \mathrm{GeV}^{2}$ (solid symbols) and $\nu=145 \mathrm{GeV}$ at $Q^{2}=35 \mathrm{GeV}^{2}$ (open symbols) ( $p_{T}$ of the hadron is integrated). The solid lines are predictions of pure energy loss calculations for pion production (see the text).

hadrons produced in a large nucleus compared to the same process on a proton, if the hadrons are formed outside the nuclear medium. It was evident from hadron production in $e^{-}+e^{+}$collisions that the fragmentation functions for light mesons, such as pions, have a very different functional form with $z$ from that of heavy mesons, such as $D$ mesons. As shown in fig. 57 (right), the heavy- $D^{0}$-meson fragmentation function has a peak while the pion fragmentation function is a monotonically decreasing function of $z$. The fact that the energy loss matches the active parton to the fragmentation function at a larger value of $z$ leads to two dramatically different phenomena in the semiinclusive production of light and heavy mesons at the EIC, as shown in fig. 58 [228]. The ratio of light meson $(\pi)$ production in $e+\mathrm{Pb}$ collisions over that in $e+d$ collisions (red square symbols) is always below unity, while the ratio of heavy meson $\left(D^{0}\right)$ production can be less than as well as larger than unity due to the difference in hadronization.

In fig. 58, simulation results are plotted for the multiplicity ratio of semi-inclusive DIS cross-sections for producing a single pion (left) and a single $D^{0}$ (right) in $e+\mathrm{Pb}$ collisions to the same produced in the $e+d$ as a function of $z$ at the EIC with two different photon energies: $\nu=35 \mathrm{GeV}$ at $Q^{2}=10 \mathrm{GeV}^{2}$ (solid line and square symbols) and $\nu=145 \mathrm{GeV}$ at $Q^{2}=35 \mathrm{GeV}^{2}$ (dashed line and open symbols). The $p_{T}$ of the observed hadrons is integrated. The ratio for pions (red square symbols) was taken from the calculation of [222], extended to lower $z$, and extrapolated from a copper nucleus to a lead nucleus using the prescription of [223]. In this model approach, pions are suppressed in $e+\mathrm{A}$ collisions due to a combination of the attenuation of pre-hadrons as well as medium-induced energy loss. In this figure, the solid lines (red: $\nu=145 \mathrm{GeV}$ and blue: $\nu=35 \mathrm{GeV}$ ) are predictions of pure energy loss calculations using the energy loss parameters of [229]. The large differences in the suppression between the square symbols and solid lines are immediate consequences of the characteristic time scale for the color neutralization and the details of the attenuation of pre-hadrons, as well as the model for energy loss. With the size of the systematic errors shown by the yellow bar on the left of the unity ratio, the multiplicity ratio of pion production at the EIC will provide an excellent and unique opportunity to study hadronization by using the nucleus as a femtometer detector.

The dramatic difference between the multiplicity ratios of $D^{0}$ meson production and that of pions, as shown in fig. 58, is an immediate consequence of the difference in the fragmentation functions shown in fig. 57 (right). The enhancement of the ratio is caused by the peak in the $D^{0}$ 's fragmentation function. The slope of the enhancement is sensitive to the amount of energy loss, or equivalently, the transport coefficient, $\hat{q}$ of cold nuclear matter, and the shape of the fragmentation function [228]. The energy loss used in the simulation is a factor of 0.35 less than that of light quarks as derived in [230] by taking into account the limited cone for gluon radiation caused by the larger charm quark mass. The solid symbols are for $x=0.1$ and $Q^{2}=10 \mathrm{GeV}^{2}$. In the same figure we also show the same type of plot but for $\nu=145 \mathrm{GeV}$ and $Q^{2}=35 \mathrm{GeV}^{2}$. The 
expected reduction in the level of pion suppression relative to $\nu=35 \mathrm{GeV}$ is visible and the shape of the $D^{0}$ data is quite different from that for $\nu=35 \mathrm{GeV}$. In addition to the $D^{0}$ meson, similar studies could be carried out with the $D_{s}^{+}$and other heavy-meson states, from which more complete information on heavy-quark energy loss could be extracted. This strong sensitivity of the shape to the value of $\nu$ will be a unique and powerful tool in the understanding of energy loss of heavy quarks in cold nuclear systems. The discovery of such a dramatic difference in multiplicity ratios between light and heavy meson production in fig. 58 at the EIC would shed light on the hadronization process and on what governs the transition from quarks and gluons to hadrons.

\subsubsection{Spatial fluctuation of parton density inside a large nucleus}

The transverse flow of particles is a key piece of evidence for the formation of a strongly interacting QGP in relativistic heavy-ion collision. It was recognized that fluctuations in the geometry of the overlap zone of heavy-ion collisions lead to some unexpected azimuthal $\phi$ modulations $v_{n}$ of particle multiplicity with respect to the reaction plane. In particular, $v_{3}$ leads to very interesting features of two-particle correlations. The initial-state density fluctuations seem to influence the formation and expansion of the QGP. An independent measurement of the spatial fluctuations of quark and gluon densities inside a large nucleus is hence critically important for understanding both, the formation of QGP in heavy-ion collisions and nuclear structure in terms of quarks and gluons.

Multiple scattering between the produced parton and the nuclear medium in semi-inclusive $e+\mathrm{A}$ collisions can broaden the transverse momentum spectrum of the produced hadron in comparison with that in corresponding $e+p$ collisions. The nuclear modification to the transversemomentum spectrum could be quantified by defining the transverse momentum broadening in terms of the azimuthal angle dependent broadening,

$$
\begin{aligned}
\Delta\left\langle p_{T}^{2}\right\rangle_{A N} & \equiv \int_{0}^{2 \pi} \mathrm{d} \phi \Delta\left\langle p_{T}^{2}(\phi)\right\rangle_{A N} \\
& \equiv \int_{0}^{2 \pi} \mathrm{d} \phi\left\langle p_{T}^{2}(\phi)\right\rangle_{A}-\int_{0}^{2 \pi} \mathrm{d} \phi\left\langle p_{T}^{2}(\phi)\right\rangle_{N}
\end{aligned}
$$

with the averaged transverse momentum squared at a given $\phi$,

$$
\left\langle p_{T}^{2}(\phi)\right\rangle_{A}=\int \mathrm{d} p_{T}^{2} p_{T}^{2} \frac{\mathrm{d} \sigma_{e A}}{\mathrm{~d} x_{B} \mathrm{~d} Q^{2} \mathrm{~d} p_{T}^{2} \mathrm{~d} \phi} / \frac{\mathrm{d} \sigma_{e A}}{\mathrm{~d} x_{B} \mathrm{~d} Q^{2}} .
$$

The azimuthal angle $\phi$ is defined as the angle between the leptonic and hadronic scattering planes in semi-inclusive DIS, as shown in Sidebar III. The measurement of the transverse momentum broadening in eq. (30) provides important information on the strength and distribution of the color fields inside the colliding nucleus and the color neutralization of the fast-moving parton, since the color

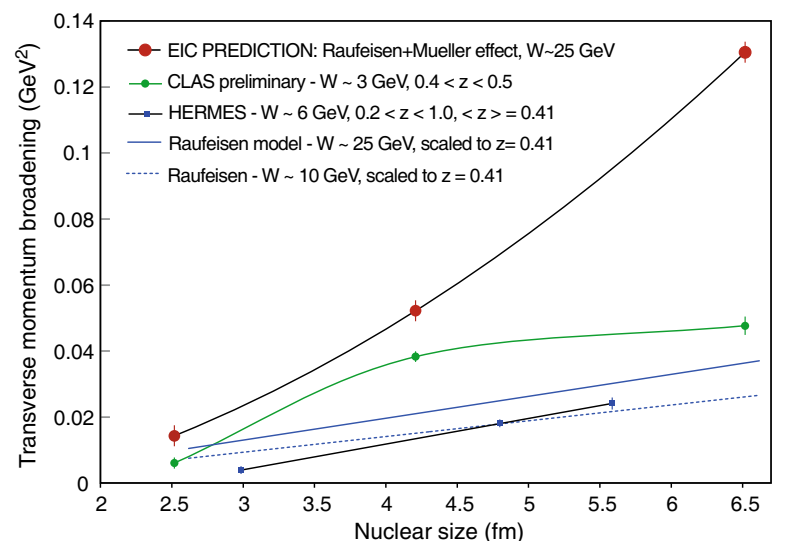

Fig. 59. Transverse momentum broadening as a function of nuclear size in $e+\mathrm{A}$ collisions as defined in eq. (30). See the text for the details.

clearly affects the interaction between the fragmenting parton and the nucleus, and hence the amount of the broadening. In addition, the transverse-momentum broadening also depends on the underlying QCD mechanism of multiple scatterings, as well as on $Q_{s}$, the typical virtuality of the scattering partons inside the nucleus. The larger $Q_{s}$ is, the broader the transverse-momentum distribution gets. However, our understanding of the fundamental QCD mechanism controlling color propagation and interaction inside a nuclear medium is still at an early stage. With its high energy and luminosity, better detector(s), and precise measurements of the transverse broadening, the EIC will enable rapid advance in our knowledge of color neutralization and multiple scattering of colored partons.

Figure 59 shows the broadening of the transversemomentum spectrum of positive pions as a function of nuclear radius for various nuclei. Existing measurements from HERMES and CLAS are shown, as well as a calculation from Raufeisen [231] who has compiled and compared the results of various theoretical approaches to the transverse-momentum broadening. In these approaches a linear dependence on the nuclear radius is obtained. As shown in fig. 59, the HERMES data exhibit a linear dependence, while the CLAS data (for which $3.7<\nu<4.3 \mathrm{GeV}$; $1.8 \leq Q^{2}<4.2 \mathrm{GeV}^{2}$; and $\left.0.4<Z_{h}<0.5\right)$ show a saturation of the broadening at large nuclear radii, which is likely related to the reduced lifetime of the colored virtual quark at the lower energies where the rescattering becomes weaker once the color of the fragmenting quark is neutralized. However, at the EIC with a much higher energy, more phase space opens up for radiation, and a qualitatively different behavior is expected [232]. As shown in fig. 59, the points, labelled for EIC from [232], predict a nonlinear increase of the broadening caused by a logarithmic enhancement of the medium-induced radiation, which contributes substantially to the broadening beyond the contribution from the elastic rescattering. In fig. 59, error bars of the EIC data points result from a PYTHIA simulation for which $x>0.1, Q^{2}>1 \mathrm{GeV},\langle z\rangle=0.41$ (matching the HERMES data), and $\langle W\rangle=25 \mathrm{GeV}$. The size of the scat- 
tering centers was taken to be that of a constituent quark for the purpose of this plot; scattering from a smaller-sized object will logarithmically enhance the size of the effect. An integrated luminosity of $10 \mathrm{fb}^{-1} / \mathrm{A}$ has been assumed, and systematic uncertainties similar to the statistical uncertainties have been employed; the two uncertainties are combined in quadrature.

In semi-inclusive DIS, the uniquely determined leptonic plane plays the role of the reaction plane in relativistic heavy-ion collisions, and helps define the azimuthal angle $\phi$ distribution of produced hadrons. Therefore, like the relativistic heavy-ion $(\mathrm{A}+\mathrm{A})$ collisions, the $\phi$-modulation of produced hadrons in SIDIS, or $v_{n}$, is well-defined on an event-by-event basis. The non-uniform spatial distribution of the scattering centers (the parton densities) inside a large nucleus could naturally generate a $\phi$-dependence of the transverse-momentum broadening of the observed hadron, which was observed by the CLAS Collaboration at Jefferson Lab [212].

With a large enough $Q$ to localize the production of a fast moving fragmenting parton at the EIC, the strength of exotic $\phi$-modulation of hadrons could shed light on the spatial fluctuation of parton densities inside a large colliding nucleus. Within the one-photon-exchange approximation for unpolarized semi-inclusive DIS, the produced hadrons naturally have the $\cos (n \phi)$ modulation with $n=1,2$, due to the interference of two scattering amplitudes with the virtual photon in different spin states. Non-vanishing exotic modulation of transverse momentum broadening on an event-by-event basis, $\Delta\left\langle p_{T}^{2}(\phi)\right\rangle_{A N} \propto$ $\cos (n \phi)$ with $n$ other than 1 and 2 , is a direct and clean evidence of spatial fluctuation of parton densities in a large colliding nucleus [233]. In addition, the $A$-dependence of $\cos (\phi)$ and $\cos (2 \phi)$ could also shed light on the spatial fluctuation of parton densities in the nucleus. The EIC could provide an independent verification and study of spatial fluctuations of parton densities inside the colliding nuclei observed in relativistic heavy ion collisions, and help us understand the initial condition of the collision to produce the QGP.

\subsection{Connections to $p+A, A+A$ and cosmic ray physics $^{13}$}

\subsubsection{Connections to $p+$ A physics}

Both $p+\mathrm{A}$ and $e+\mathrm{A}$ collisions can provide excellent information on the properties of gluons in the nuclear wave-functions. It is therefore only logical to compare the strengths and weaknesses of the two different programs in exploring the saturation regime.

In the beginning of the RHIC era, the $d+\mathrm{Au}$ program was perceived as merely a useful baseline reference for the heavy-ion program. It very soon turned out that, due to a wise choice of colliding energy, RHIC probes the transition region to a new QCD regime of gluon saturation. While only marginal hints of non-linear effects were

\footnotetext{
13 Conveners: Yuri Kovchegov and Thomas Ullrich.
}

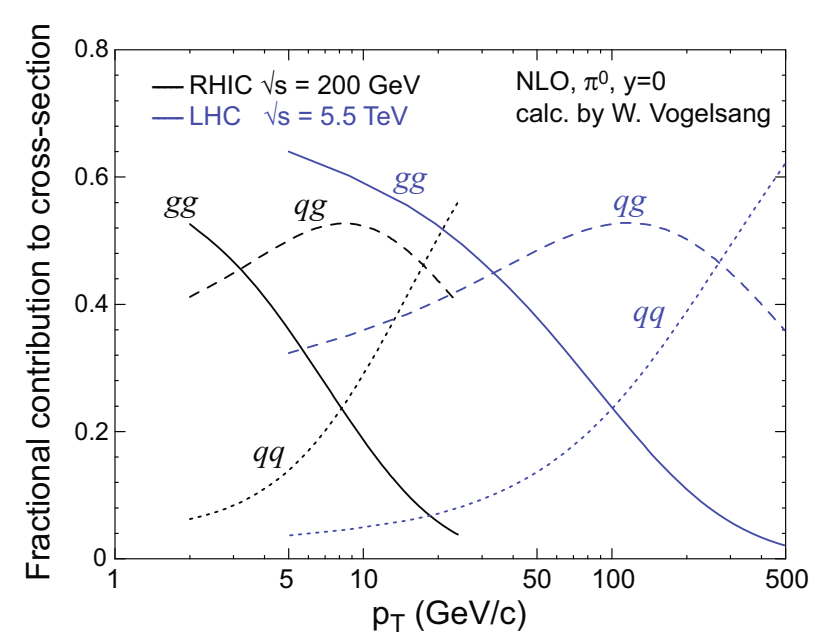

Fig. 60. Fractional contributions from $g g, q g$, and $q q$ scattering processes to pion production at mid-rapidity $p+p$ collisions at RHIC (black) and LHC (blue).

observed in DIS experiments at HERA [234], it is fair to say that very tantalizing hints for gluon saturation were observed in $d+\mathrm{Au}$ collisions at RHIC [195, 196, 235-237]. In the $p+\mathrm{A}$ program at the LHC, these effects should be even more pronounced as the data from forward rapidities become available. While $p+\mathrm{A}$ and $p+p$ colliders provide superior access to the low- $x$ region, they also have some severe disadvantages that impede systematic studies of the saturation phenomena that we will describe below.

As shown in fig. 60 , in $p+p$ collisions at mid-rapidity at RHIC and the LHC, the bulk of particles produced originate from processes involving gluons. This is a simple manifestation of the dominance of gluons at low- $x$ in hadrons (see fig. 34). While it is unlikely that saturation phenomena are observed at RHIC energies in $p+p$ collisions due to the small values of $Q_{s}$ even at the lowest accessible $x$, the amplified $Q_{s}$ scale in $p+\mathrm{A}$ collisions opens the experimentally accessible range where saturation effects become detectable. The relation between rapidity $y$ and transverse momentum $p_{T}$ of the final-state partons/particles with mass $m$ and their fractional longitudinal momenta $x_{1,2}$ is $x_{1,2}=e^{ \pm y} \sqrt{\left(p_{T}^{2}+m^{2}\right) / s}$. Hence, at mid-rapidity $(y=0)$ at RHIC, only particle production with very small $p_{T}$ will be sensitive to the saturation region in parton densities while at the LHC the region of transverse momenta will be much larger. At RHIC, saturation effects are largely absent at central rapidities but become measurable at large forward rapidities (that is, for particles coming out close to the incoming proton or deuteron direction with $y=2-4$ corresponding to small $\left.x_{2}\right)$.

First hints for the onset of saturation in $d+\mathrm{Au}$ collisions at RHIC have been observed by studying the rapidity dependence of the nuclear modification factor, $R_{d A u}$, as a function of $p_{T}$ for charged hadrons [235] and $\pi^{0}$ mesons [237], and more recently through forward-forward hadron-hadron correlations $[195,196]$. 


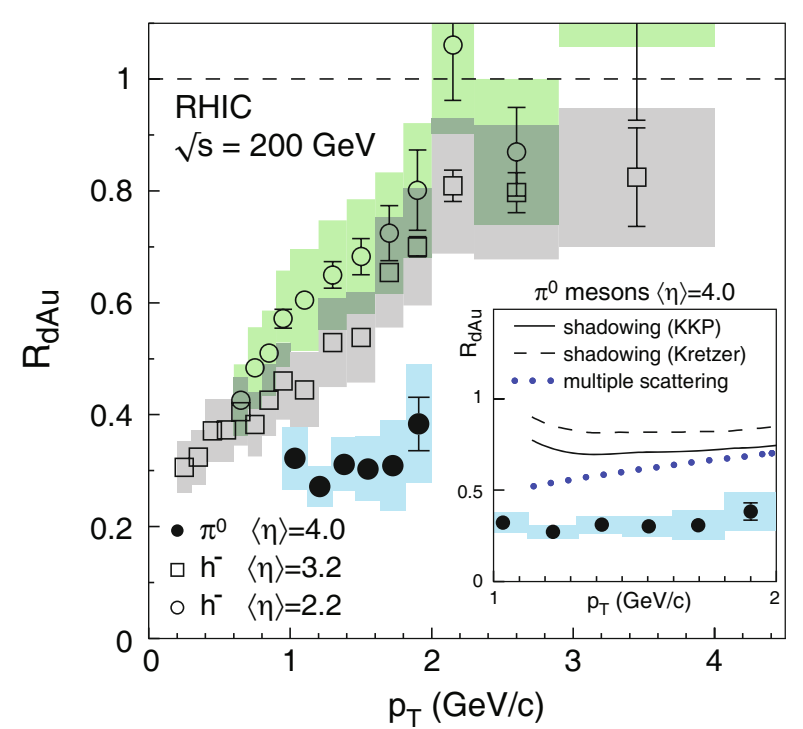

Fig. 61. The nuclear modification factor $\left(R_{d A u}\right)$ versus $p_{T}$ for minimum bias $d+\mathrm{Au}$ collisions measured at RHIC. The solid circles are for $\pi^{0}$ mesons [237], the open circles and boxes are for negative hadrons [235]. The error bars are statistical, the shaded boxes are point-to-point systematic errors. (Inset) $R_{d A u}$ for $\pi^{0}$ mesons compared with pQCD calculations based on collinear factorization. Note that none of the curves can describe the data.

The nuclear modification factor for a $p+\mathrm{A}$ collision is defined by

$$
R_{p A}=\frac{1}{N_{\text {coll }}} \frac{\mathrm{d} N_{p A} / \mathrm{d}^{2} p_{T} \mathrm{~d} y}{\mathrm{~d} N_{p p} / \mathrm{d}^{2} p_{T} \mathrm{~d} y},
$$

where $\mathrm{d} N / \mathrm{d}^{2} p_{T} \mathrm{~d} y$ is the produced hadron multiplicity in a given region of phase space while $N_{\text {coll }}$ is the number of binary nucleon-nucleon collisions. The nuclear modification factor $R_{p A}$ is equal to 1 in the absence of collective nuclear effects. Figure 61 shows $R_{d A u}$ versus $p_{T}$ for minimum bias $d+$ Au collisions for charged hadrons measured by the BRAHMS experiment [235] and $\pi^{0}$ mesons by STAR [237]. While the inclusive yields of hadrons $\left(\pi^{0}\right.$ mesons) at $\sqrt{s}=200 \mathrm{GeV}$ in $p+p$ collisions generally agree with pQCD calculations based on DGLAP evolution and collinear factorization, in $d+\mathrm{Au}$ collisions, the yield per binary collision is suppressed with increasing $\eta$, decreasing to $\sim 30 \%$ of the $p+p$ yield at $\langle\eta\rangle=4$, well below shadowing and multiple scattering expectations. The $p_{T^{-}}$ dependence of the $d+\mathrm{Au}$ yield is found to be consistent with the gluon saturation picture of the Au nucleus (e.g., CGC model calculations [239-244]) although other interpretations cannot be ruled out based on this observable alone [216, 245, 246].

Recent result from the $p+\mathrm{Pb}$ scattering experiments at the LHC appear to confirm this picture. Figure 62 depicts the data for $R_{p P b}$ of charged particles reported by ALICE Collaboration as compared to different theoretical models. The data is the same in all three sub-panels. Saturation models, whose predictions are depicted in the top panel of fig. 62 , do a good job in describing the data, though other

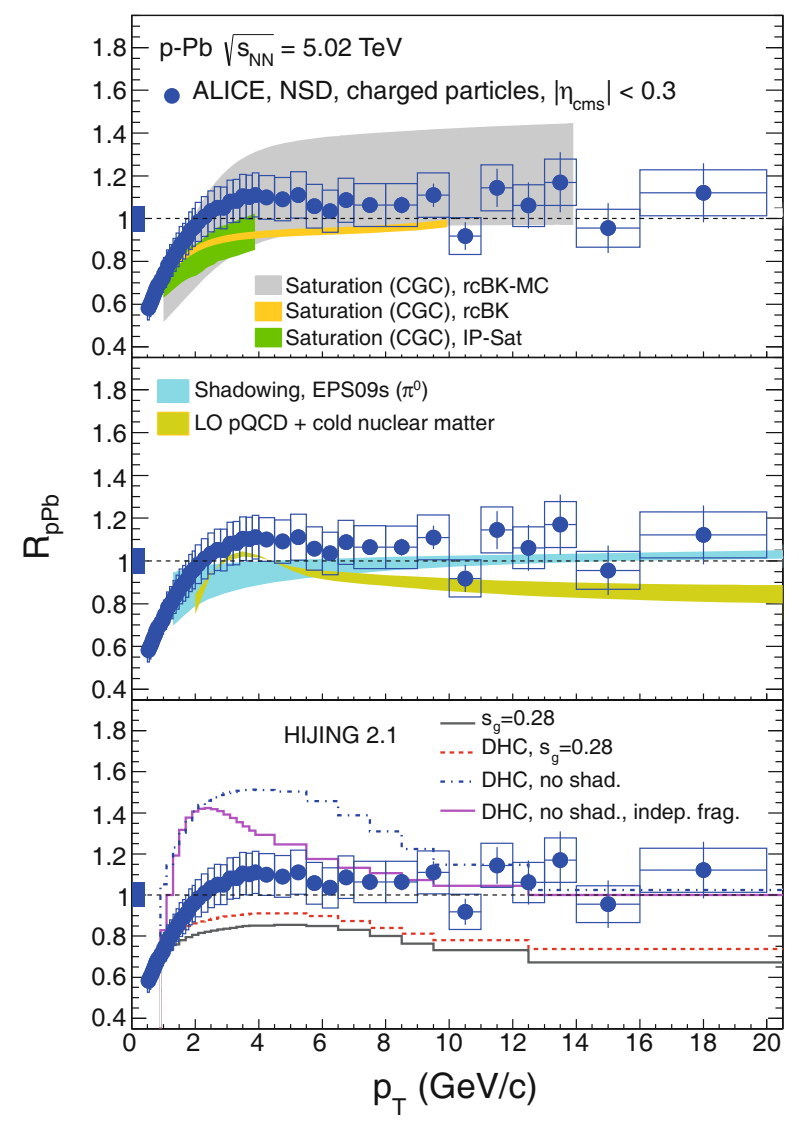

Fig. 62. The nuclear modification factor $\left(R_{p P b}\right)$ versus $p_{T}$ for charged particles produced in $p+\mathrm{Pb}$ collisions at LHC [238] compared to various theoretical models.

models' predictions, most notably that of EPS09 shown in the middle panel, also describe the data well.

A more powerful technique than single inclusive measurements is the use of two-particle azimuthal correlations, as discussed in sect. 3.2.2. In collinear factorization-based pQCD at leading order, particle production in high-energy hadronic interactions results from the elastic scattering of two partons $(2 \rightarrow 2$ scattering) leading to back-to-back jets. When high- $p_{T}$ hadrons are used as jet surrogates, we expect the azimuthal correlations of hadron pairs to show a peak at $\Delta \phi=0$, and a "back-to-back" peak at $\pi$. When the gluon density increases, the basic dynamics for the particle production is expected to change. Instead of elastic $2 \rightarrow 2$ scattering, particle production can proceed by the interaction of a probe parton from the proton (deuteron) beam with multiple gluons from the heavy-ion beam. At sufficiently high gluon densities, the transverse momentum from the fragments of the probing parton may be compensated by several gluons with lower $p_{T}$. Two-particle azimuthal correlations are expected to show a broadening of the back-to-back peak (loss of correlation: $2 \rightarrow$ many processes) and eventually to disappear. In the CGC framework, the hadronic wave-function is saturated as a consequence of gluon recombination. At very low values of the $x$ of the probed gluons, the occupation 

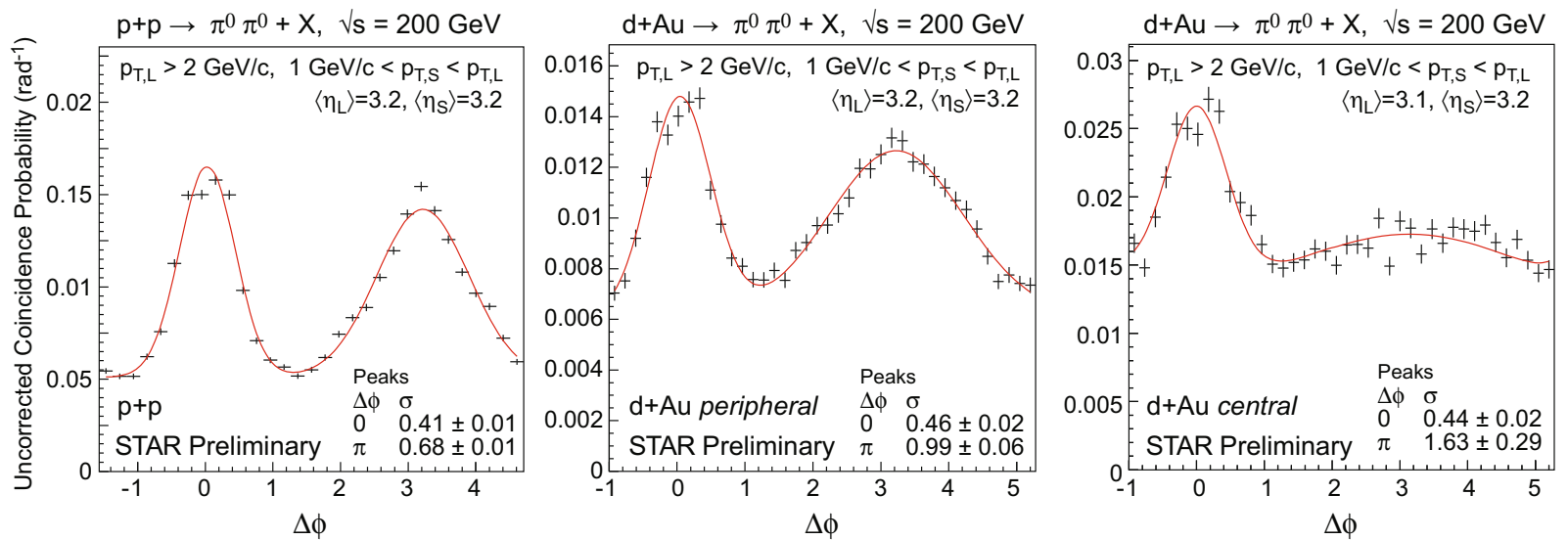

Fig. 63. Di-hadron correlations measured at forward rapidities at RHIC: the uncorrected coincidence signal plotted versus the azimuthal angle difference between two forward neutral pions in $p+p$ collisions (left) compared to peripheral (center) and central $d+\mathrm{Au}$ collisions (right) [196]. Data are shown with statistical errors and fit with a constant plus two Gaussian functions (in red).

numbers become large and the probe scatters coherently off the dense gluon field of the target, which recoils collectively, leading to a modification in $\Delta \phi[191]$.

Figure 63 shows the (efficiency uncorrected) probability to find an associated $\pi^{0}$ given a trigger $\pi^{0}$, both in the forward region measured by the STAR detector. The coincidence signal versus azimuthal angle difference between the two pions in $p+p$ collisions (left) compared to peripheral (middle) and central $d+$ Au collisions (right) is shown [196] (see also fig. 50 for a similar measurement and the related discussion, along with [195]). All the distributions present two signal components, surmounting a constant background representing the underlying event contribution (larger in $d+\mathrm{Au}$ ). The near-side peak represents the contribution from pairs of pions belonging to the same jet. It is not expected to be affected by saturation effects. The away-side peak represents the back-to-back contribution to the coincidence probability, which should disappear in going from $p+p$ to $d+\mathrm{Au}$ if saturation sets in [191]. The data show that the width of the near-side peak remains nearly unchanged from $p+p$ to $d+\mathrm{Au}$, and particularly from peripheral to central $d+\mathrm{Au}$ collisions. Central $d+$ Au collisions show a substantially reduced away side peak that is significantly broadened. Again, pQCD calculations based on linear DGLAP evolution without coherent multiple scattering fail to describe this observation, while those including non-linear effects describe the data considerably well $[240,247,248]$. This measurement represents the strongest hint yet for saturation phenomena and also indicates that the kinematic range of the EIC is well suited to explore saturation physics with great precision.

One of the most important results from the $\mathrm{LHC} p+\mathrm{Pb}$ program is the observation of the "ridge" correlation in high-multiplicity $p+\mathrm{Pb}$ collisions (see fig. 64). The "ridge" is a di-hadron correlation which is very broad in rapidity $(\Delta \eta)$ and very narrow in the azimuthal angle $(\Delta \phi)$. The near-side "ridge" $(\Delta \phi \approx 0)$ was originally discovered in heavy-ion collisions at RHIC [249-252]. With the advent of the LHC experimental program, it was also seen

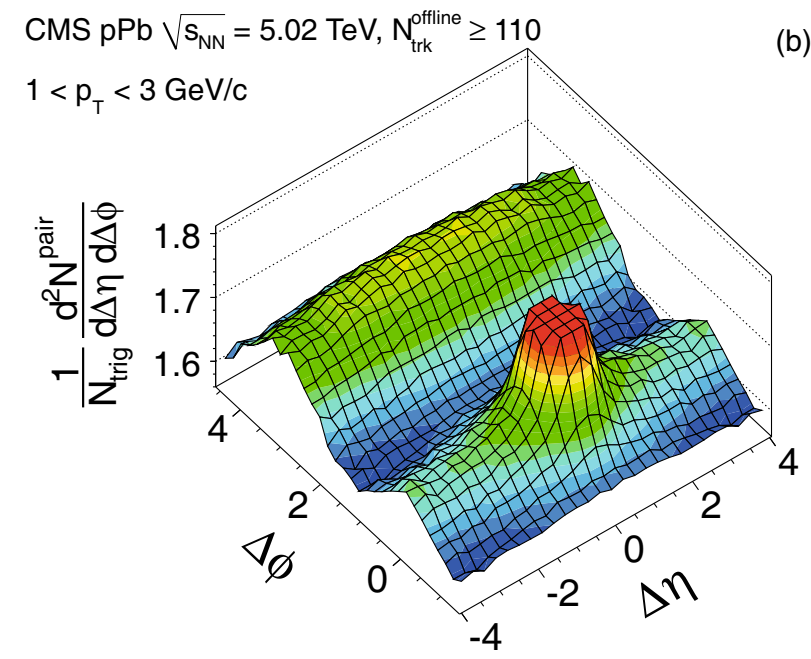

Fig. 64. The two-particle correlation function in highmultiplicity $p+\mathrm{Pb}$ collisions as a function of $\Delta \phi$ and $\Delta \eta$ reported by the CMS Collaboration [254]. The "ridge" structure is seen as a correlation near $\Delta \phi=0$ stretching over many units of rapidity $\Delta \eta$.

in high-multiplicity $p+p[253]$ and $p+\mathrm{Pb}[254-256]$ collisions. A simple causality argument [257] indicates that the long-range rapidity correlation in the "ridge" is due to dynamics in the early stages of the collisions, and hence may possibly be due to the saturation effects. While saturation effects may also explain the narrow azimuthal structure of the "ridge" correlation [257], in heavy-ion collisions the azimuthal shape of the correlation is likely to be strongly affected by the final-state QGP effects.

The dynamical origin of the azimuthal shape of the "ridge" in $p+p$ and $p+\mathrm{Pb}$ collisions is less clear, since collective QGP effects are usually not expected in such systems. A quadrupole azimuthal anisotropy with symmetric peaks at $\Delta \phi \approx 0$ and $\Delta \phi \approx \pi$ was predicted in [257-261] based on CGC physics and was experimentally confirmed 
in [255], suggesting a saturation origin of the correlation. A quantitative comparison of CGC theory to data was made in [262]. However, at the moment all saturationbased explanations of the "ridge" appear to predict the correlation function which is expandable into a Fourier series over even cosine harmonics, $\cos (2 n \Delta \phi)$, whereas the data presents clear evidence of odd harmonics as well, even in high-multiplicity $p+\mathrm{Pb}$ collisions [256], similar to those generated by hydrodynamic expansion of QGP in $\mathrm{A}+\mathrm{A}$ collisions on top of the event-by-event fluctuations in the initial conditions (see sect. 3.4.2 below). At the same time, preliminary measurements of the system size [263] appear to be in contradiction with the hydrodynamic interpretation. Clearly the jury is still out regarding the origin of the "ridge" in high-multiplicity $p+p$ and $p+\mathrm{Pb}$ collisions: it is possible that both CGC and hydrodynamic effects are at play. While LHC can reach down to very low values of $x$, it is possible that the ability to study small- $x$ physics and saturation at $\mathrm{LHC} p+\mathrm{Pb}$ experiments is somewhat blunted by the final-state interactions. The final-state effects should not be present at an $e+\mathrm{A}$ collider, which should allow for a cleaner probe of low- $x$ dynamics.

Although the results of the $d+\mathrm{Au}$ program at RHIC and the $p+\mathrm{Pb}$ program at LHC show tantalizing evidence of saturation phenomena, alternative explanations for each of the individual observations exist. The unambiguous ultimate proof of existence of saturation can only come from an $e+\mathrm{A}$ collider. While in $e+\mathrm{A}$ collisions the probe (the electron) is point-like and structureless, in $p+\mathrm{A}$ collisions, one has to deal with a probe whose structure is almost as complex as that of the target nucleus to be studied. The EIC's usefulness as a gluon "microscope" is somewhat counterintuitive since electrons do not directly interact with gluons. However, the presence and dynamics of the gluons in the ion will modify the precisely understood electromagnetic interaction of the electron with quarks in ways that allow us to infer the gluon properties. Deeply inelastic $e+$ A collisions are dominated by one photon exchange (see Sidebar I). The photon could interact with one parton to probe parton distributions, as well as multiple partons coherently to probe multi-parton quantum correlations. One of the major advantages of DIS is that it allows for the direct, model-independent, determination of the momentum fraction $x$ carried by the struck parton before the scattering and $Q^{2}$, the momentum transferred to the parton in the scattering process. Only the control of these variables ultimately will allow us a precise mapping of the gluon distributions and their dynamics.

One may wonder whether physics similar to what one can probe at an EIC could be studied in the Drell-Yan process in a $p+\mathrm{A}$ collider. Due to crossing symmetry, the Drell-Yan process can be related to DIS [264] with the invariant mass of the di-lepton pair $M^{2}$ playing the role of $Q^{2}$. Owing to the very broad reach in $x$ and $M^{2}, p+$ A collisions at RHIC and even more so at the LHC clearly have significant discovery potential for the physics of strong color fields in QCD. However, the di-lepton signal in $p+\mathrm{A}$ is contaminated by the leptons resulting from decays of heavy-flavor hadrons, such as $J / \psi$, up to a rather large invariant masses of $M^{2}=16 \mathrm{GeV}^{2}$ and even beyond [265]. This contamination does not allow one to cleanly probe the saturation region of $M^{2}<16 \mathrm{GeV}^{2}$. To avoid hadronic decay background one may study large values of the net transverse momentum $p_{T}$ of the pair. However, this would also push one away from the lower- $p_{T}$ saturation region.

Ultimately it will be the combination of strong $p+$ A and $e+\mathrm{A}$ programs, each providing complementary measurements, that will answer the questions raised above in full.

\subsubsection{Connections to ultra-relativistic heavy-ion physics}

Measurements over the last decade in heavy-ion collision experiments at RHIC indicate the formation of a strongly coupled plasma of quarks and gluons (sQGP). Striking results include: i) the strong collective flow of all mesons and baryons, and especially that of heavy charm quarks, and ii) the opaqueness of the hot and dense medium to hadron jets up to $p_{\perp} \sim 20 \mathrm{GeV}$.

This sQGP appears to behave like a "near-perfect fluid" with a ratio of the shear viscosity to entropy density, $\eta / s$, approaching zero [266-270]. Recent experiments at the LHC, with substantially higher energies and thus a hotter and longer lived plasma phase, confirm this picture [271].

Despite the significant insight that the QGP is a strongly correlated nearly perfect fluid, little is understood about how the QGP is created and what its properties are. Qualitative questions that the heavy-ion community would like to answer include how the dynamics of gluons in the nuclear wave functions generates entropy after the collision, what the properties and dynamics of the pre-equilibrium state are, why the thermalization of the system occurs rapidly, and whether the system is fully or only partially thermalized during its evolution. Furthermore, though it is widely accepted that the QGP medium is a strongly correlated one, it is less clear whether the coupling is weak or strong. In the weak-coupling scenario, the strongly correlated dynamics are generated by the scales that characterize the electric and magnetic sectors of the hot fluid. In the strongly-coupled scenario, progress has been made by exploiting the Anti-de Sitter space/Conformal Field Theory (AdS/CFT) correspondence $[272,273]$ of weakly coupled gravity (which is calculable) to strongly coupled supersymmetric Yang-Mills theory with many features in common with QCD.

Quantitative questions the heavy-ion community would like to answer include determining the shear viscosity of the medium averaged over its evolution, measuring the values of other transport coefficients such as the bulk viscosity and the heavy-quark diffusion coefficient, and perhaps most importantly, identifying the equation of state of finite-temperature QCD medium. Some of these questions can be addressed in numerical lattice QCD computations. It is still not entirely clear how these results can be cross-checked and improved upon in the environment of a rapidly evolving and incredibly complex heavy-ion event. 
Despite the significant progress achieved in the qualitative understanding of several aspects of this matter, there is still no comprehensive quantitative framework to understand all the stages in the creation and expansion of the hot and dense QGP medium. In the following we outline how an EIC can contribute to a better understanding of the dynamics of heavy-ion collisions, from the initial formation of bulk partonic matter to jet quenching and hadronization that probe the properties of the sQGP.

\section{Initial conditions in $A+A$ collisions}

Understanding the dynamical mechanisms that generate the large flow in heavy-ion collisions is one of the outstanding issues in the RHIC program. Hydrodynamic modeling of RHIC data is consistent with the system rapidly thermalizing at times of around $1-2 \mathrm{fm} / c$ after the initial impact of the two nuclei [274-276]. These hydrodynamic models are very sensitive to the initial preequilibrium properties of the matter formed immediately after the collision of the two nuclei.

Our current understanding, based on the CGC framework, suggests that the wave-functions of the nuclei, due to their large occupancy, can be described as classical fields, as was explained above. Therefore, at the leading order, the collision can be approximated by the collision of "shock waves" of classical gluon fields [277,278] resulting in the production of non-equilibrium gluonic matter. It is generally believed that the instability and consequent exponential growth of these intense gluon fields would be the origin of early thermalization [279,280], though the exact mechanism for the process is not completely understood. Alternatively, within the strong-coupling paradigm, thermalization in heavy ion collisions is achieved very rapidly, and there has been considerable recent work in this direction [281, 282].

The properties of the nuclear wave functions will be studied in great detail in $e+\mathrm{A}$ collisions. They promise a better understanding of the initial state and its evolution into the sQGP. Specifically, the saturation scale $Q_{s}$, which can be independently extracted in $e+\mathrm{A}$ collisions, sets the scale for the formation and thermalization of strong gluon fields. Saturation effects of these low- $x$ gluon fields affect the early evolution of the pre-QGP system in heavy-ion collisions. Their spatial distribution governs the eccentricity of the collision volume and this affects our understanding of collective flow and its interpretation profoundly. However, the features of these gluon fields - their momentum and spatial distributions at energies relevant for RHIC - are only vaguely known. More detailed information of relevance to the properties of the initial state (such as the spatial distributions of gluons and sea quarks) and thereby improved quantitative comparisons to heavy ion data, can be attained with an EIC.

The high-energy wave functions of nuclei can be viewed as coherent superpositions of quantum states that are "frozen" configurations of large numbers of primarily gluons. How these states decohere, produce entropy and subsequently interact is clearly essential to a deep understanding of high-energy heavy-ion collisions. Remarkably,

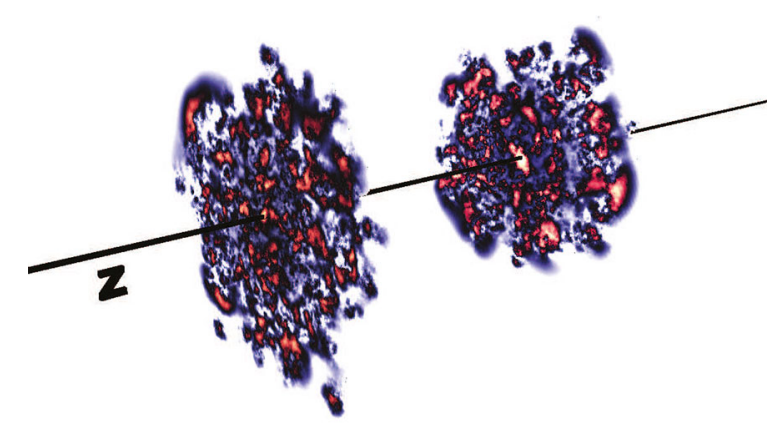

Fig. 65. The spatial distribution of gluon fields of the incoming nuclei for a collision of lead ions at $\sqrt{s}=2760 \mathrm{GeV}$. The colors - from blue to red - denote increasing strength of gluon correlations.

models based on the CGC framework manage to describe particle production in $\mathrm{A}+\mathrm{A}$ collisions over a broad range of energies and centralities extraordinary well. These models are constrained by HERA inclusive and diffractive DIS data on $e p$ collisions, and the limited fixed target $e+$ A DIS data available. One such model is the IP-Sat model [174,283] (Model-I from sect. 3.2.1) Another saturation model (Model-II from sect. 3.2.1) is based on BK nonlinear evolution including the running-coupling corrections (rcBK) [180-183] and the impact parameter independence [284].

The IP-Sat model can be used to construct nucleon color charge distributions event-by-event. Convoluting this with Woods-Saxon distributions of nucleons enables one to construct Lorentz contracted two-dimensional nuclear color charge distributions of the incoming nuclei event-by-event. Such a nuclear color charge density profile is shown in fig. 65 for a heavy-ion collision. The scale of transverse event-by-event fluctuations in fig. 65 is $1 / Q_{s}$, not the nucleon size. The resulting model $[287,288] \mathrm{em}-$ ploys the fluctuating gluon fields generated by the IPSat model to study the event-by-event evolution of gluon fields. Here, the corresponding energy density distributions vary on the scale $1 / Q_{s}$ and are therefore highly localized (as shown in the left panel of fig. 66).

The right panel in fig. 66 shows data for the centrality dependence of charged particle production for heavy-ion collisions at $\sqrt{s_{N N}}=200 \mathrm{GeV}$ and $2760 \mathrm{GeV}$ compared to both Model-I (IP-Sat with fluctuations) and Model-II (based on rcBK evolution). Both models do an excellent job of describing the data (note that Model-II is a prediction). The pale bands shown in this figure are the event-byevent fluctuations of the multiplicity in the Model-I. The successful descriptions of the energy and centrality dependence of multiplicity distributions at RHIC and the LHC are strong indications that the $\mathrm{CGC}$ provides the right framework for entropy production. Therefore, a fuller understanding of the small- $x$ formalism promises to enable us to separate these initial state effects from final-state entropy production during the thermalization process and thereby constrain mechanisms (by their centrality and energy dependence) that accomplish this. 

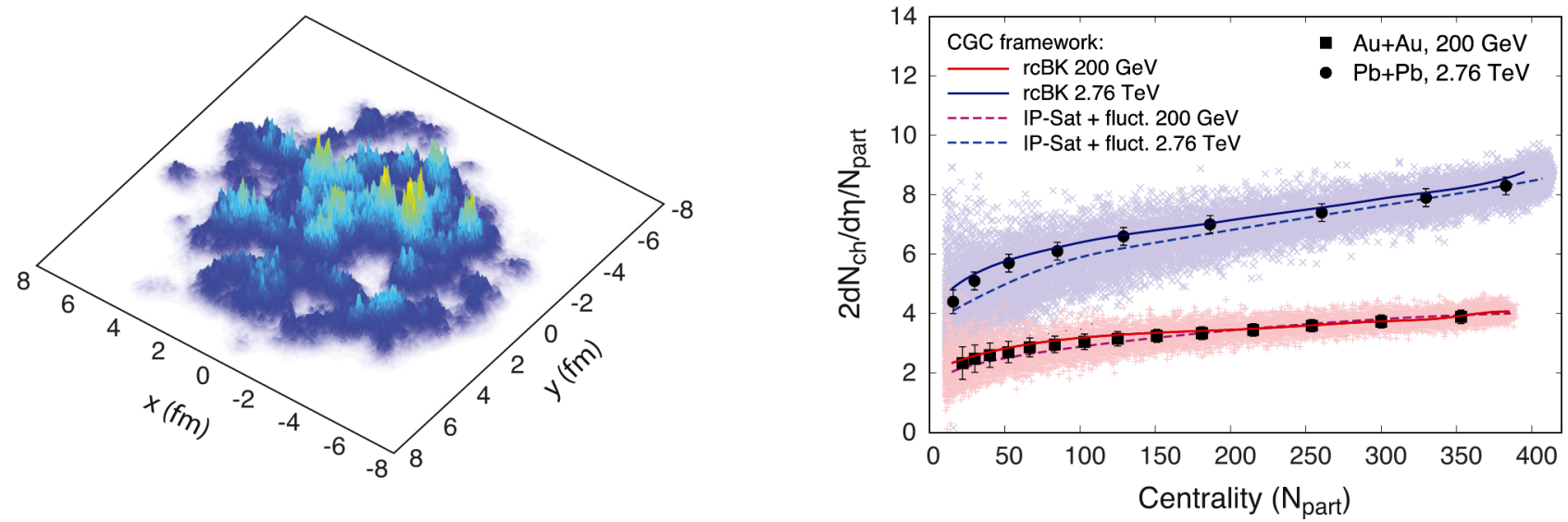

Fig. 66. Left panel: the spatial variation of the energy density in a single heavy-ion event (based on IP-Sat model with fluctuations). The variations occur on distance scales $1 / Q_{s}$, much smaller than the nucleon size. Right panel: the centrality dependence of the multiplicity at $\sqrt{s_{N N}}=200 \mathrm{GeV}$ and $2760 \mathrm{GeV}$. (AuAu [285] data from RHIC, PbPb data [286] from LHC.). The experimental data are compared to results from two model realizations in the CGC framework. Solid curves represent the results from $k_{T}$-factorization with running-coupling BK unintegrated gluon distributions [284] (Model-II from before) while dashed curves represent the result in the IP-Sat model with fluctuations [287] (Model-I). The pale blue (LHC) and pink bands (RHIC) denote the referring range of event-by-event values of the single inclusive multiplicity.
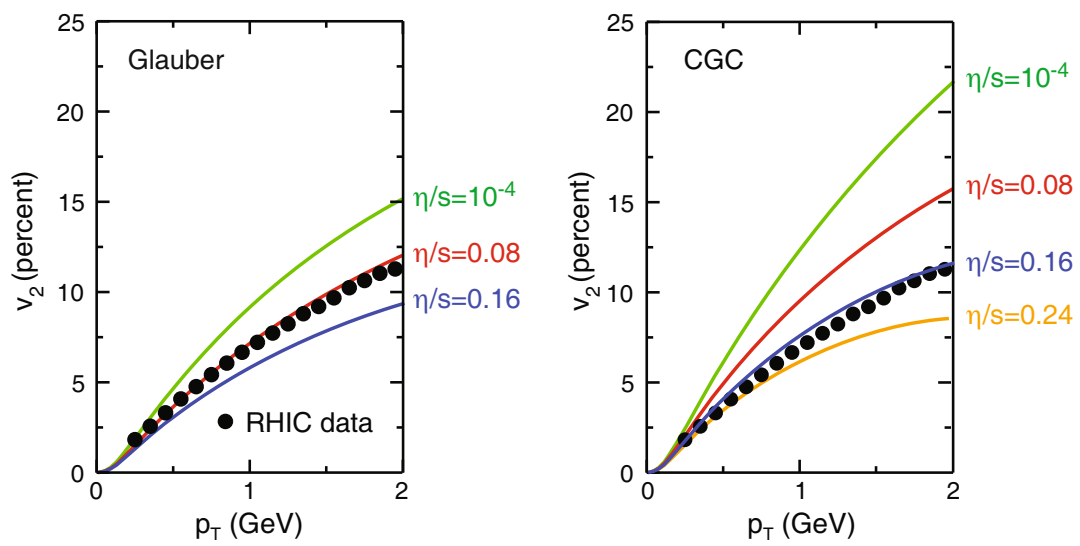

Fig. 67. A comparison of data and theoretical predictions using viscous relativistic hydrodynamics for $v_{2}^{h}\left(p_{T}\right)$ (right) with Glauber-like initial conditions (left) or a simplified implementation of CGC physics (KLN) model (right). Figures adapted from $[276]$.

The other bulk quantity very sensitive to the properties of the initial state is the collective flow generated in heavy-ion collisions. A useful way to characterize flow [289] is through measured harmonic flow coefficients $v_{n}$, defined through the expansion of the azimuthal particle distribution as

$$
\frac{\mathrm{d} N}{\mathrm{~d} \phi}=\frac{N}{2 \pi}\left(1+\sum_{n} 2 v_{n} \cos (n \tilde{\phi})\right)
$$

where $v_{n}\left(p_{T}\right)=\langle\cos (n \tilde{\phi})\rangle$, with $\langle\cdots\rangle$ denoting an average over particles in a given $p_{T}$ window and over events in a given centrality class, and $\tilde{\phi}=\phi-\psi_{n}$ with the event plane angle $\psi_{n}=\frac{1}{n} \arctan \frac{\langle\sin (n \phi)\rangle}{\langle\cos (n \phi)\rangle}$. Spatial eccentricities, extant at the instant a hydrodynamic flow description becomes applicable, are defined, e.g., for the second harmonic as $\varepsilon_{2}=\left\langle y^{2}-x^{2}\right\rangle /\left\langle y^{2}+x^{2}\right\rangle$, where now $\langle\cdots\rangle$ is the energy density-weighted average in the trans- verse $x-y$ plane. These are in turn converted to momentum space anisotropies by hydrodynamic flow. How efficiently this is done is a measure of the transport properties of the strongly coupled QCD matter such as the shear and bulk viscosities. Early flow studies focused on the second flow harmonic coefficient $v_{2}$, which is very large at RHIC and the LHC, and particularly sensitive to the ratio of the shear-viscosity-to-entropy-density ratio $\eta / s$. In fig. 67 , we show $v_{2}$ for a Glauber model used in hydrodynamic simulations (left) and the Kharzeev-Levin-Nardi CGC (KLN-CGC) model [290] (right). The eccentricity $\varepsilon_{2}$ in the Glauber model has a weaker dependence on collision centrality relative to the KLN-CGC model, and therefore requires a lower $\eta / s$ to fit the data. The value of $\eta / s$ for the Glauber eccentricity in this model study is equal to $1 / 4 \pi$ in natural units $[291,292]$ conjectured to be a universal bound for strongly-coupled liquids based on applications of the Anti-de Sitter space/Conformal Field 


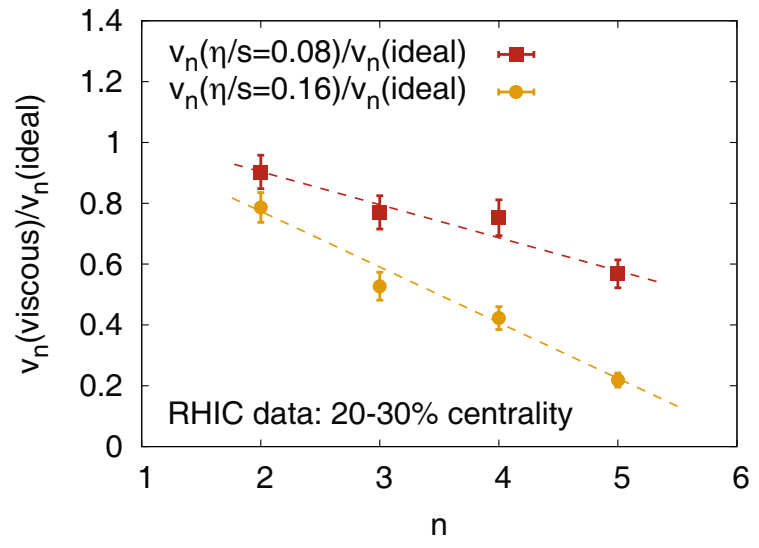

Fig. 68. The ratio of charged hadron flow $v_{n}$ harmonics from viscous hydrodynamic simulations and from ideal hydrodynamics [293] (lines only to guide the eye). The ratio is shown for two different values of $\eta / s$. Higher harmonics are substantially more affected by shear viscosity than $v_{2}$.

Theory (AdS/CFT) correspondence $[272,273]$. The KLNCGC value on the other hand gives a number that is twice as large as this prediction.

Experimental and theoretical developments can help settle what is the true value of $\eta / s$, and in particular, potentially provide essential information on its temperature dependence. Interestingly, the effect of $\eta / s$ on each of the $v_{n}$ harmonics is different. This is shown strikingly in comparisons of results for the $v_{n}$ moments from event-by-event viscous hydrodynamic simulations relative to equivalent ideal hydrodynamic simulations in fig. 68 .

The figure shows the ratio of viscous to ideal moments [293] for $n=2, \ldots, 5$ for the previously discussed values of $\eta / s$. The damping of the higher moments $v_{n}$ is quite dramatic with increasing $\eta / s$.

The results shown are for the Glauber model, and the values for $v_{2,3,4}$ are in good agreement with available RHIC data [294] for $\eta / s=0.08$. In contrast, the CGC-KLN model, which we saw fits data for the larger $\eta / s=0.16$, does poorly with $v_{3}$ because of the damping effect noted. The poor agreement of the CGC-KLN model with the higher $v_{n}$ moments can be traced primarily to the absence of event-by-event color charge fluctuations we discussed previously. The IP-Sat model with fluctuations $[287,288]$ includes these and the results for the moments are closer to the Glauber model that is tuned to fit the data.

It is here that we see that the EIC might have significant additional impact on bulk observables in $\mathrm{A}+\mathrm{A}$ collisions. This is, of course, in addition to the absolutely crucial input of establishing the saturation paradigm of entropy production, extracting information on the energy and centrality dependence of $Q_{s}$ and providing information on gluon correlations that may influence thermalization and long range rapidity correlations. The measurement of $\mathrm{d} \sigma / \mathrm{d} t$ in diffractive $e+\mathrm{A}$ collisions (see sect. 3.2.2 and fig. 54) allows for a clean determination of the spatial gluon density on average and will help constrain the scale and magnitude of event-by-event fluctuations of color charge densities. Inelastic vector meson production can further constrain the spatial extent of these eventby-event fluctuations [295]. Hadronic multiplicity fluctuations, along with the di-hadron correlation, would also allow one to pinpoint the dynamical origin of the energy density fluctuations in heavy ion collisions. Such direct access to spatial information is unique to $e+\mathrm{A}$ collisions (in contrast to $p+\mathrm{A}$ collisions) and therefore can only be provided by an EIC.

\section{Energy loss and hadronization}

The dramatic suppression of high-transversemomentum (high- $p_{T}$ ) hadrons discovered at RHIC is important evidence for the production of a dense medium in nuclear collisions. It is commonly accepted that partonic energy loss could be the main cause of the observed suppression of hadrons at a sufficiently high $p_{T}$ (much larger than the hadron mass) assuming that the hadronization of a high- $p_{T}$ hadron is taking place outside the medium. However, if color neutralization of the hadronization process starts inside the medium, partonic energy loss might not be the only mechanism contributing to the observed suppression, and additional forms of suppression could be relevant. Furthermore, the experimental evidence for suppression of hadrons composed of heavy quarks is quite complex. So far, the observed suppression could not be explained with the pure partonic energy loss treatment, even though a description of heavy quarks should be a straightforward extension of the approach for light quarks, taking into account the more important role of collisional losses. These observations strongly imply that there are, at the very least, missing elements in our understanding of what needs to be included in describing the observed suppression of high- $p_{T}$ hadrons, and require us to better understand the partonic energy loss and time-evolution of hadronization, and to explore other independent measurements which can test the suppression mechanism.

If energy loss of a colored fast-moving parton is the sole suppression mechanism, the inclusive hadron suppression at high $p_{T}$ in $\mathrm{A}+\mathrm{A}$ collisions could be represented by a single parameter - the jet transport coefficient $\hat{q}$ of the medium, defined as the average transverse momentum squared acquired by a parton traversing a medium per unit distance traveled. However, the extraction of $\hat{q}$ from the A + A data hardly provides a clean test of the energy loss mechanism because of the complexity of the created medium's dynamics, which includes, in particular, the first $1-2 \mathrm{fm} / c$ after the collision where the medium is in a non-equilibrium stage proceeding towards thermalization. Furthermore, because of the complexity of partonic kinematics in hadronic and nuclear collisions, the suppression of inclusive hadron production does not provide a simple connection of the momentum fractions $x_{1}$ and $x_{2}$ of colliding partons and the fraction $z_{h}$ of the fragmenting parton momentum carried by the produced hadron; these parameters are crucial in determining the mediuminduced partonic energy loss. 

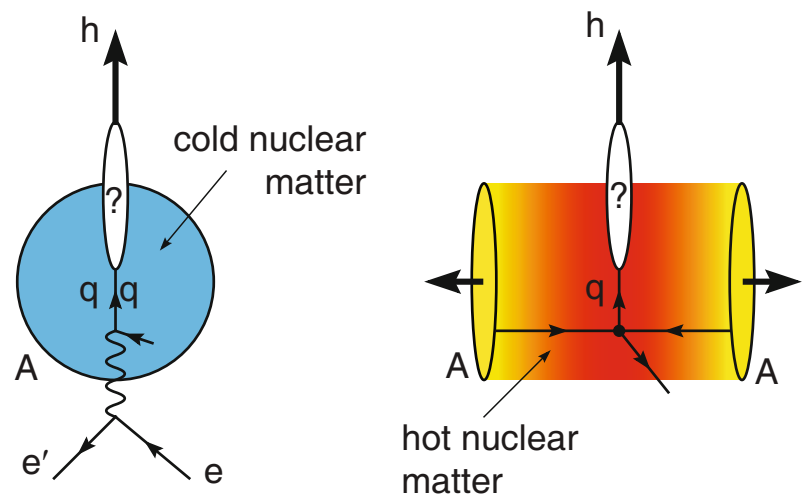

Fig. 69. Cartoon showing a similarity of the kinematics and geometry in production of hadrons in a hot matter created in $\mathrm{A}+\mathrm{A}$ collisions and in SIDIS on nuclei.

High-energy hadron production in electron-ion collisions could offer an alternative and cleaner way to study the mechanisms of energy loss and in-medium hadronization of energetic virtual partons moving through nuclear medium. Semi-inclusive deep inelastic processes (SIDIS, see sect. 2) can be used as a testing ground for the suppression mechanism of high- $p_{T}$ hadron production seen in the nuclear collisions, as illustrated in fig. 69, where the similarity of the kinematics and geometry in hadron production in SIDIS and A + A collisions was presented.

The transverse momentum $p_{T}$ of the detected hadrons in $\mathrm{A}+\mathrm{A}$ collisions varies up to $10 \mathrm{GeV}$ and higher at RHIC and up to about $100 \mathrm{GeV}$ at the LHC. Available data from SIDIS in fixed target experiments, such as HERMES [296] and CLAS [297], cover only a small part of the hadron momentum range observed at RHIC and the LHC. As demonstrated in sect. 3.3.2, the coverage could be significantly extended by SIDIS measurements at a future EIC. The path lengths in the cold nuclear matter and hot medium are similar, of the order of the nuclear radius. However, SIDIS on nuclear targets allows to test suppression models in much more specific and controlled conditions. The nuclear density does not vary with time, its value and spatial distribution are well known, while the probe is characterized by the virtual photon's energy $\nu$ and the photon's four-momentum squared $Q^{2}$ are also uniquely determined (see Sidebar I). At the leading order of the strong interaction, the momentum of the hadronizing quark, as well as the fractional energy $z_{h}$ of the detected hadron, are effectively measured.

Accurate measurements of different observables, like the magnitude of suppression and broadening at different $\nu, z_{h}$ and $Q^{2}$ with different nuclei should provide a stringent test for the models of energy loss and in-medium hadronization. If the suppression is dominated by the partonic energy loss, these measurements would help constrain the value of the jet quenching parameter $\hat{q}$ of a known medium. This parameter is central to the energy loss studies in A + A collisions: its value for hot nuclear matter in the early stages of the collision is presently narrowed down to the range of $1 \mathrm{GeV}^{2} / \mathrm{fm}$ to $10 \mathrm{GeV}^{2} / \mathrm{fm}$. The cold nuclear matter experiments at an EIC would help further pinpoint the value of this important parameter.

Furthermore, at an EIC, for the first time one will be able to study open charm and open bottom meson production in $e+\mathrm{A}$ collisions, as well as the in-medium propagation of the associated heavy quarks: these measurements would allow one to fundamentally test high-energy QCD predictions for partonic energy loss, and confront puzzling measurements of heavy flavor suppression in the QGP at RHIC (see sect. 3.3.2).

With a wide energy coverage, the EIC could be an excellent machine to study the space-time development of hadronization by varying the energy and virtuality of the probe - the exchanging virtual photon in SIDIS. As discussed in sect. 3.3.2, the color neutralization of the fragmenting quark could take place inside the nuclear medium to form the so-called "pre-hadron" state, which is a name for a state of partons with zero net color but with the same quantum numbers of a hadron that the state is about to transmute into. The "pre-hadron" state represents an intermediate stage of the hadronization process from an energetic single parton produced in a hard collision to the hadron observed in the detector. This stage is expected to exist from general theoretical considerations, but it is likely non-perturbative. If it does exist, the interaction of the "pre-hadron" state with nuclear medium should certainly be different from that of a colored and fast-moving single parton. As indicated in fig. 58, the EIC is capable to distinguish the suppression caused by a purely partonic energy loss from that involving a "pre-hadron" stage.

\section{Ultra-peripheral collisions}

Ultra-peripheral collisions (UPC) are defined as interactions among two nuclei that are separated by impact parameters larger than the sum of their radii. The ions do not interact directly with each other and move essentially undisturbed along the beam direction. Due to the coherent action of all the protons in the nuclei, the electromagnetic fields are very strong and the resulting flux of equivalent photons is large $\left(\propto Z^{2}\right)$; the otherwise dominant hadronic interactions are strongly suppressed. The only possible interaction is electromagnetic involving a long-range photon exchange. A photon stemming from the electromagnetic field of one of the two colliding nuclei can penetrate into the other nucleus and interact with one or more of its hadrons, giving rise to photon-nucleus collisions [298]. Ultra-peripheral heavy-ion collisions are an important, albeit kinematically limited alternative, which is being used to study QCD dynamics until an EIC becomes a reality.

A special focus of the UPC program at RHIC and LHC are photo-nuclear reactions involving the exclusive production of heavy vector mesons such as $J / \psi$ and $\psi(2 \mathrm{~S})$ [300]. They provide a good tool for evaluating the behavior of the gluon distribution functions at low $x$, since the photoproduction cross-section scales at leading order as the square of the gluon distribution $G\left(x, \mu^{2}\right)$; the scale $\mu$ is typically approximated by $M_{V} / 2$, where $M_{V}$ is the mass of the vector meson. However, as pointed out in sect. 3.2.2, 


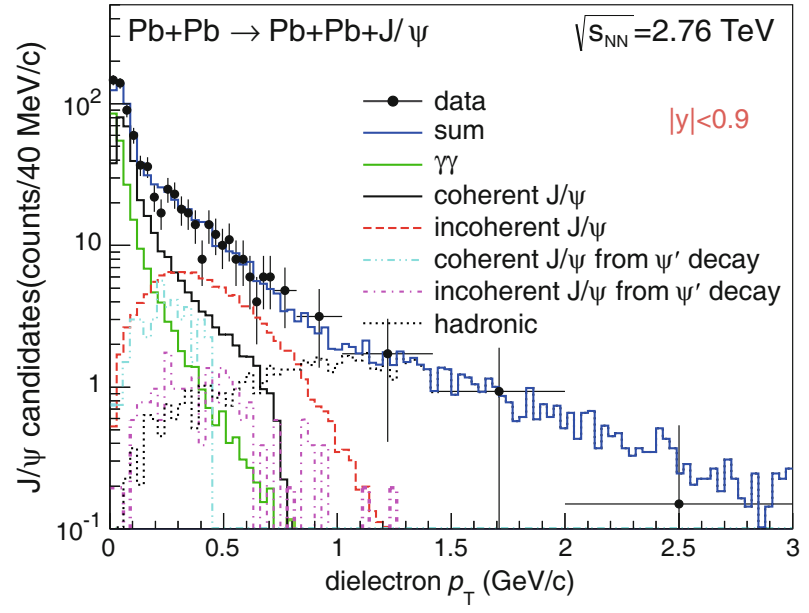

Fig. 70. Plot of the $p_{T}$-distribution of $J / \psi$ mesons produced in ultra-peripheral $\mathrm{Pb}+\mathrm{Pb}$ collisions at $\sqrt{s_{N N}}=2.76 \mathrm{TeV}$ as measured by ALICE. [299]. Overlaid are coherent and incoherent components derived from Monte Carlo simulations.

heavy vector mesons are less sensitive to gluon saturation than lighter vector mesons such as $\phi$ and $\rho$, whose production cross-sections are much harder to measure in UPCs.

The exclusive photoproduction can be either coherent, where the photon couples coherently to almost all the nucleons, or incoherent, where the photon couples to a single nucleon. Coherent production is characterized by low transverse momentum of vector mesons $\left(\left\langle p_{T}\right\rangle \approx 60 \mathrm{MeV} / c\right.$ at LHC) where the nucleus normally does not break up. Incoherent production, corresponding to quasi-elastic scattering off a single nucleon, is characterized by a somewhat higher transverse momentum $\left(\left\langle p_{T}\right\rangle \approx 500 \mathrm{MeV} / c\right.$ at LHC). Unlike the $e+\mathrm{A}$ collisions with their wide coverage in $Q^{2}$, vector meson production in UPCs is limited to vanishingly small $Q^{2}$, much smaller than most of the other momentum scales in the problem. The value of $Q^{2}$ is inversely proportional to the square of the impact parameter between the nuclei, such that the photon is quasi-real. The momentum transfer $t$ can be approximated by $t \approx-p_{T}^{2}$.

Figure 70 shows as an example the $p_{T}$-distribution of $J / \psi$ mesons produced in ultra-peripheral $\mathrm{Pb}+\mathrm{Pb}$ collisions at $\sqrt{s_{N N}}=2.76 \mathrm{TeV}$ measured by the ALICE Collaboration [299]. The clear peak at low $p_{T}$ is mainly due to coherent interactions, while the tail extending out to $1 \mathrm{GeV} / c$ comes from incoherent production.

In general, measurements of ultra-peripheral collisions at RHIC and LHC energies can provide useful insights into gluon densities at low $x$. They lack, however, the wide kinematic coverage in $Q^{2}$ and the precise knowledge of the relevant kinematic variables that will be available at an EIC.

\subsubsection{Connections to cosmic ray physics}

Decisive evidence in favor of parton saturation, which could be uncovered at an EIC, would also have a profound impact on the physics of Cosmic Rays.

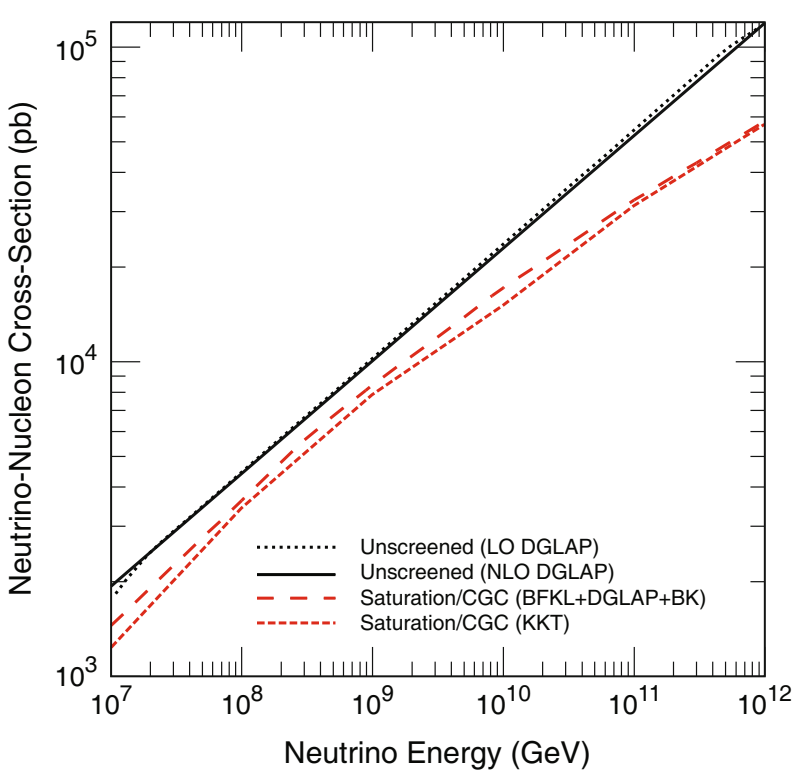

Fig. 71. Predictions of several models with (red) and without (black) parton saturation (CGC) physics for the cross-sections for neutrino-nucleon scattering at high energies as calculated in [301-303]. The KKT saturation model is defined in [244]. Saturation effects appear to lower the neutrino-nucleon crosssection at very high energies in agreement with general expectations of saturation taming the growth of the gluon numbers.

The sources of the observed ultra-high energy cosmic rays must also generate ultra-high-energy neutrinos. Deep inelastic scattering of these neutrinos with nucleons on Earth is very sensitive to the strong interaction dynamics. This is shown in fig. 71 for the cross-sections for neutrinonucleon scattering plotted as a function of the incident neutrino energy for several models. As we argued above, the experiments at an EIC would be able to rule out many of the models of high-energy strong interactions, resulting in a more precise prediction for the neutrino-nucleon cross-section, thus significantly improving the precision of the theoretical predictions for the cosmic ray interactions. The improved precision in our understanding of strong interactions will enhance the ability of the cosmic-ray experiments to interpret their measurements accurately and will thus allow them to uncover new physics beyond the Standard Model of particle physics.

Saturation physics that is likely to be discovered and studied at an EIC has other important connections with cosmic-ray physics. One key question concerns the nuclear composition of ultra-high-energy cosmic rays: are they made out of protons or out of heavier nuclei? At energies above about $10^{16} \mathrm{eV}$, the low cosmic-ray flux forces us to rely on indirect measurements of the composition. These indirect measurements necessarily depend on the modeling of the hadronic showers that the cosmic-rays produce. Variables such as the depth of the shower maximum $\left(X_{\max }\right)$ in the atmosphere and the muon content of the showers depend strongly on the hadronic modeling.

The Pierre Auger Collaboration has measured the depth of shower maxima in air showers with energies above 

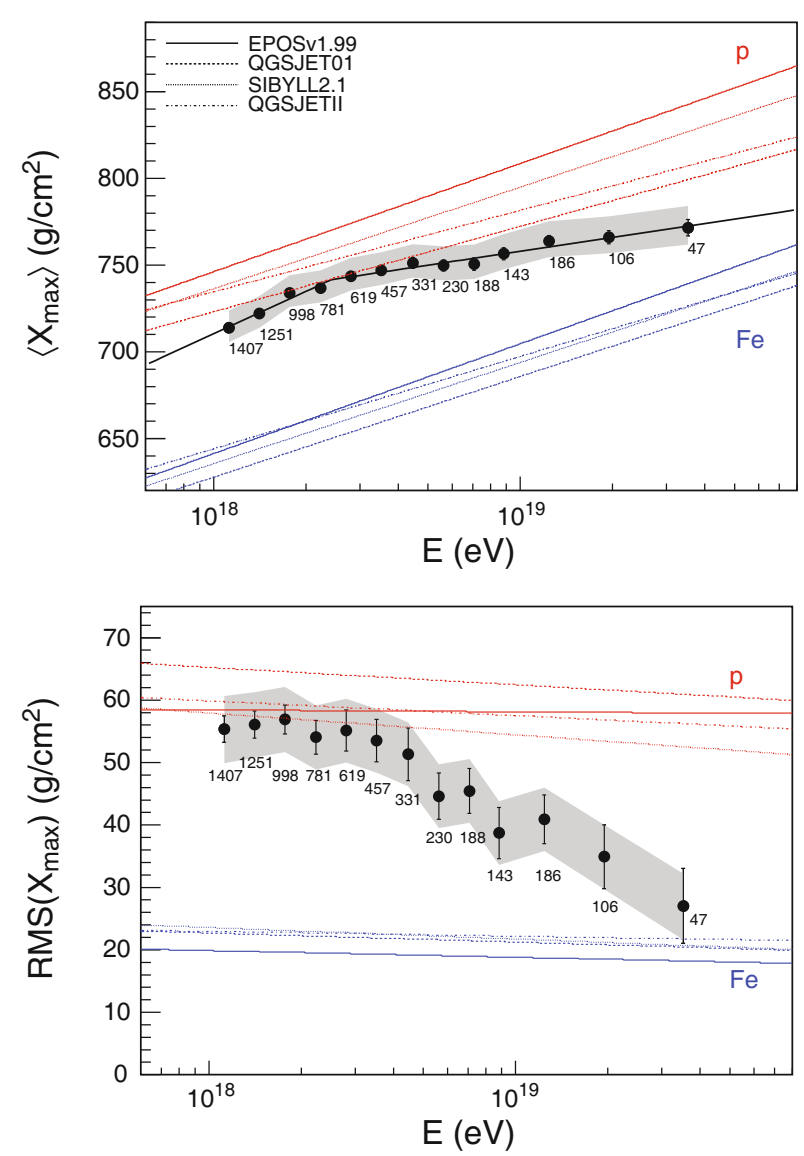

Fig. 72. The data on the atmospheric depth of the cosmic-ray shower maximum $X_{\max }$ (upper panel) and on its RMS (lower panel) as a function of the cosmic-ray energy $E$ reported by the Pierre Auger Observatory [304]. The error bars reflect the statistical uncertainty, while the bands depict the systematic uncertainty. The numbers next to the data points indicate the number of events in each data bin. The solid lines represent predictions of various Monte Carlo simulations for the cosmic ray being a proton $(p)$ and an iron nucleus $(\mathrm{Fe})$.

$10^{18} \mathrm{eV}$ [304] shown here in fig. 72. At energies below $10^{18.4} \mathrm{eV}$, they see a composition with a constant elongation rate (the slope of $X_{\max }$ plotted versus the cosmic-ray energy $\left.E_{c r}, \mathrm{~d} X_{\max } / \mathrm{d} E_{c r}\right)$ at a position that is consistent with a composition that is largely protons. However, as one can see from the upper panel of fig. 72, at higher energies, there is a significant shift in elongation rate, and, by an energy of $10^{19.4} \mathrm{eV}$, the depth $X_{\max }$ is more consistent with an all-iron composition [304]. At the same time, the root mean square (RMS) variation in the position of $X_{\max }$ (plotted in the lower panel of fig. 72) drops by a factor of two, also consistent with a change in composition. This is a rather abrupt change of composition in one decade of energy; an alternate possibility is that there is a shift in hadronic physics, such as the onset of saturation. The EIC could shed light on this possibility.

At somewhat lower energies, the IceCube Collaboration has measured the production of high-energy $(\approx$ $1 \mathrm{TeV}$ ) and high- $p_{T}$ (roughly $p_{T}>2 \mathrm{GeV} / c$ ) muons in cosmic-ray air showers [305], and needs to interpret the data using modern $\mathrm{pQCD}$, again with a view to probing the cosmic-ray composition. These forward muons come from the collision of a high- $x$ parton in the incident cosmic ray with a low- $x$ parton in the nitrogen/oxygen target in the atmosphere. Saturation will alter the distribution of low- $x$ partons in the target, and so must be considered in the calculations. EIC data is needed to pin down this possible saturation effect.

\section{Possibilities at the luminosity frontier: physics beyond the Standard Model ${ }^{14}$}

\subsection{Introduction}

It is natural to ask whether the envisioned machine parameters of the EIC could enable new discoveries in the broad subfield of Fundamental Symmetries (FS), which addresses one of the overarching goals of nuclear physics, namely, the exploration of the origin and evolution of visible matter in the early universe. The theoretical and experimental studies in this subfield are complementary to those of particle physics and cosmology. Indeed, a broader categorization of the full range of initiatives that encompass the FS goals falls under the titles "Energy Frontier", "Cosmic Frontier" and "Intensity/Precision Frontier".

The FS subfield of nuclear physics is part of the intensity/precision frontier, the specific primary goal of which is the study of electroweak interactions of leptons and hadrons with progressively higher sensitivity. By comparing the measured interaction amplitudes with theoretical predictions within the framework of the Standard Model (SM) of strong, weak and electromagnetic interactions, insights are gained into the symmetries and interactions of matter in the universe at its earliest moments of existence, indirectly accessing energy scales similar to, and sometimes beyond the reach of, the highest energy accelerators.

The EIC offers a unique new combination of experimental probes given the high center-of-mass energy, high luminosity and the ability to polarize the electron and hadron beams. Electron-hadron collisions would be analyzed by a state-of-the-art hermetic detector package with high efficiency and resolution. In this section, we explore new FS measurements that become possible with these capabilities, the physics impact of potential measurements, and the experimental requirements to enable the measurements.

Electroweak interaction studies at the EIC can also be used to probe novel aspects of nucleon structure via measurements of spin observables constructed from weak interaction amplitudes mediated by the $W$ and $Z$ bosons. Indeed, some parity-violating observables become accessible that have never before been measured. These measurements are considered in detail in sect. 2.2 along with other fundamental observables that probe the longitudinal spin structure of the nucleon.

${ }^{14}$ Conveners: Krishna Kumar and Michael Ramsey-Musolf. 


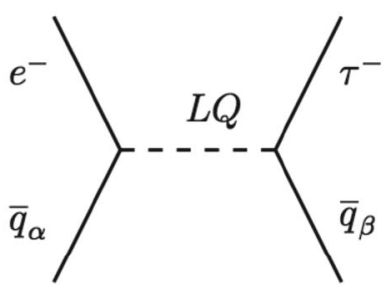

$s$-channel

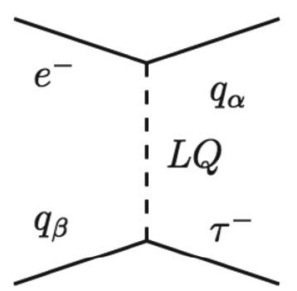

$u$-channel

$$
F=0
$$

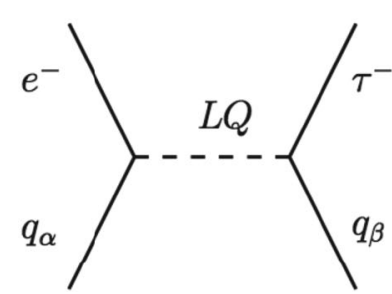

$s$-channel

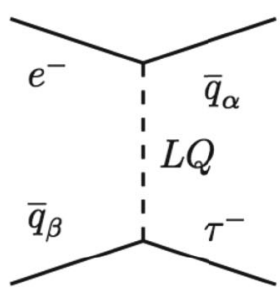

$u$-channel

$|F|=2$

Fig. 73. Feynman diagrams for $e \rightarrow \tau$ scattering processes via leptoquarks, which carry fermion number $F=3 B+L$ equal to 0 or $\pm 2[311]$.

\subsection{Specific opportunities in electroweak physics}

\subsubsection{Charged lepton flavor violation}

With the discovery of neutrino oscillations, we now know that lepton flavor is not a conserved quantity in fundamental interactions. It is natural to ask whether lepton flavor non-conservation can be observed in charged lepton interactions. In addition, the implication that neutrinos have mass leads to the fundamental question of whether neutrinos are their own anti-particles (Majorana neutrinos) which could have profound implications for the origin of the matter-antimatter asymmetry in the universe. Speculative new theories of the early universe that predict Majorana neutrinos often also predict observable rates of charged lepton flavor violation (CLFV). Searches for CLFV are thus one of the most sensitive accelerator-based low-energy probes of the dynamics of the early universe and the physics of the smallest length scales, in a manner complementary to searches for new physics at the energy frontier at the Large Hadron Collider.

The most sensitive CLFV searches to date have come from searches for the neutrinoless conversion of stopped muons to electrons in nuclei, searches for the rare decay of a free muon to an electron and photon, and searches for the rare decay of a kaon to an electron and muon. The limits from these processes, though extremely sensitive, all involve the $e \leftrightarrow \mu$ transition. Speculative CLFV theories can predict enhanced rates for $e \leftrightarrow \tau$ transitions. Existing limits for the $e \leftrightarrow \tau$ transition come from searches for rare $\tau$ decays at the high luminosity $e^{+} e^{-}$colliders at a center-of-mass energy from 5 to $10 \mathrm{GeV}$, the so-called $B$ factories.

In lepton-hadron interactions, one could search for the rare cases where an electron converts to a muon or tau lepton, or a muon converts to a tau lepton. However, this is impossible to observe due to large and irreducible background in fixed target experiments. The only successful such searches for $e \rightarrow \tau$ transitions have been carried out at the HERA electron-hadron collider experiments ZEUS and H1. In a collider environment, the event topology for rare signal events can be differentiated from conventional electroweak deep inelastic scattering (DIS) events [306-308]
The CLFV process could be mediated by a hypothesized new heavy boson known as a leptoquark, which carries both lepton and baryon quantum numbers and appears naturally in many SM extensions such as Grand Unified Theories, supersymmetry, and compositeness and technicolor models (for a concise review, see [309]). Figure 73 shows the Feynman diagrams that could be responsible for the CLFV transition that might be observed at an EIC. The most recent published search by $\mathrm{H} 1$ finds no evidence for CLFV $e \rightarrow \tau$ transitions [211], which can in turn be converted to a limit on the mass and the couplings of leptoquarks in specific SM extensions [310].

A high-energy, high-luminosity EIC, with 100 to 1000 times the accumulated luminosity of HERA experiments would allow a large increase in sensitivity. A recent study has shown that an EIC, with $90 \mathrm{GeV}$ center-of-mass energy, could surpass the current limits with an integrated luminosity of $10 \mathrm{fb}^{-1}$ [311]. The study also showed that the EIC could compete or surpass the updated leptoquark limits from rare CLFV tau decays for a subset of quark flavor-diagonal couplings. A follow-up study beyond this, including knowledge of inefficiencies from the $\mathrm{H} 1$ and ZEUS collaborations for $\tau$ reconstruction, indicates that these estimates are too optimistic by a factors of $10-20$, thus requiring $100-200 \mathrm{fb}^{-1}$ luminosity integrated over the EIC lifetime [2]. At the highest possible luminosities envisioned for the EIC, these luminosities are deemed achievable. Over the lifetime of the EIC, the $e \rightarrow \tau$ reach would thus be comparable to the reach of rare $\tau$ decays at future high-luminosity super- $B$-factories.

It must be emphasized that the unambiguous observation of a CLFV process would be a paradigm-shifting discovery in subatomic physics, with wide-ranging implications for nuclear physics, particle physics and cosmology. It is quite possible that future potential discoveries at the energy and cosmic frontiers could make CLFV searches at the EIC even more compelling.

\subsubsection{Precision measurements of weak neutral current couplings}

A comprehensive strategy to indirectly probe for new high-energy dynamics via sensitive tests of electroweak interactions at the intensity frontier must also include pre- 
cision measurements of flavor-diagonal weak neutral current interactions mediated by the $Z$ boson. For electronhadron interactions at $Q^{2} \ll M_{Z}^{2}$, weak neutral current amplitudes are accessed via parity violation since pseudoscalar observables, sensitive to weak-electromagnetic interference terms, can be constructed from the product of vector and axial-vector electron and quark electroweak currents. The parity-violating part of the electron-hadron interaction at $Q^{2} \ll M_{Z}^{2}$ can be given in terms of phenomenological couplings $C_{i j}$

$$
\begin{aligned}
\mathcal{L}^{P V}= & \frac{G_{F}}{\sqrt{2}}\left[\bar{e} \gamma^{\mu} \gamma_{5} e\left(C_{1 u} \bar{u} \gamma_{\mu} u+C_{1 d} \bar{d} \gamma_{\mu} d\right)\right. \\
& \left.+\bar{e} \gamma^{\mu} e\left(C_{2 u} \bar{u} \gamma_{\mu} \gamma_{5} u+C_{2 d} \bar{d} \gamma_{\mu} \gamma_{5} d\right)\right]
\end{aligned}
$$

with additional terms as required for the heavy quarks. Here $C_{1 j}\left(C_{2 j}\right)$ gives the vector (axial-vector) coupling to the $j$-th quark.

Within the SM context, each coupling constant is precisely predicted since they are all functions of the weak mixing angle $\sin ^{2} \theta_{W}$. Under the assumption that the recently discovered scalar resonance at the Large Hadron Collider $[312,313]$ is the SM Higgs boson, the value of the weak mixing angle is now known to better than $0.03 \%$. Over the past two decades, the $C_{1 i}$ couplings have been measured with steadily improving precision in tabletop atomic parity violation experiments and in fixed target parity-violating electron scattering experiments, most recently at Jefferson Laboratory (JLab) [314]. Comparing these measurements to SM predictions has produced strong constraints on new high-energy dynamics, such as limits on TeV-scale heavy $Z^{\prime}$ bosons and certain classes of interactions in supersymmetric theories, in a manner complementary to direct searches at colliders $[315,316]$. This is an active field with new experimental tools under development, as described in recent reviews [317-319].

At the EIC, the availability of high-luminosity collisions of polarized electrons with polarized ${ }^{1} \mathrm{H}$ and ${ }^{2} \mathrm{H}$ would allow the construction of parity-violating observables that are sensitive to all four semi-leptonic coupling constants introduced above. The observable with the best sensitivity to cleanly measure coupling constants without significant theoretical uncertainty is $A_{P V}$ in $e_{-}^{2} \mathrm{H}$ collisions. $A_{P V}$ is constructed by averaging over the hadron polarization and measuring the fractional difference in the deep inelastic scattering (DIS) rate for right-handed vs. left-handed electron bunches.

The collider environment and the hermetic detector package at high luminosity will allow precision measurements of $A_{P V}$ over a wide kinematic range. In particular, the EIC will provide the opportunity to make highly precise measurements of $A_{P V}$ at high values of the 4momentum transfer $Q^{2}$, and in the range $0.2 \lesssim x \lesssim 0.5$ for the fraction of the nucleon momentum carried by the struck quark, such that hadronic uncertainties from limited knowledge of parton distribution functions and higher-twist effects are expected to be negligible.

By mapping $A_{P V}$ as a function of $Q^{2}$ and the inelasticity of the scattered electron $y$ (something that is very challenging to do in fixed-target experiments), a clean separa-

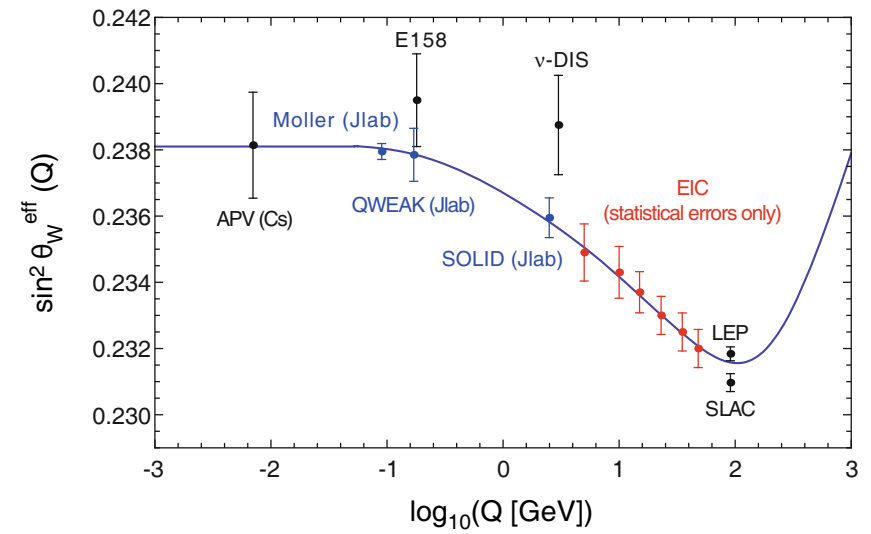

Fig. 74. Projected statistical uncertainties on the $\sin ^{2} \theta_{W}$ in a series of $Q^{2}$ bins $\left(\sqrt{s}=140 \mathrm{GeV}, 200 \mathrm{fb}^{-1}\right.$.) The black points are published results while the blue points are projections from the JLab program.

tion of two linear combination of couplings namely $2 C_{1 u^{-}}$ $C_{1 d}$ and $2 C_{2 u}-C_{2 d}$ will become feasible as a function of $Q^{2}$. Thus, at the highest luminosities and center-of-mass energies envisioned at the EIC, very precise measurements of these combinations can be achieved at a series of $Q^{2}$ values, providing an important and complementary validation of the electroweak theory at the quantum loop level. Figure 74 shows a first estimate of projected uncertainties on the weak mixing angle extracted from such a dataset [2], for a center-of-mass energy of $140 \mathrm{GeV}$ and an integrated luminosity of $200 \mathrm{fb}^{-1}$. The effects of radiative corrections and detector effects need to be considered in the future to further refine this study.

A unique feature of DIS $A_{P V}$ measurements is the sensitivity to the $C_{2 i}$ coupling constants that involve the amplitudes with axial-vector quark currents. While the couplings are kinematically accessible at large scattering angle measurements in fixed target elastic electron scattering, axial-hadronic radiative correction uncertainties cloud the interpretation of the measurements in terms of fundamental electroweak physics. Parity-violating DIS using ${ }^{2} \mathrm{H}$ is the only practical way to measure one combination accurately, namely $2 C_{2 u}-C_{2 d}$. A recent measurement at $6 \mathrm{GeV}$ at JLab made the first non-zero measurement of this combination [320], and a new experiment has been proposed at $11 \mathrm{GeV}$ to constrain this combination to better than $10 \%$. At the highest envisioned luminosities, the EIC would offer the opportunity to further improve on this constraint by a further factor of 2 to 3 .

One example of the importance of achieving sensitive constraints on the $C_{2 i}$ couplings is depicted in fig. 75 , which shows how a heavy $Z^{\prime}$ boson (predicted in many SM extensions) could introduce an additional amplitude and induce a deviation in the measured $C_{2 i}$ couplings [321]. A remarkable feature of this amplitude is the fact it is sensitive to the $Z^{\prime}$ boson even in the case that it might not couple to leptons (so-called lepto-phobic $Z^{\prime}$ ). The limits on the existence of such bosons from other precision weakneutral-current measurements as well as from colliders is very weak because all signatures require non-zero lepton$Z^{\prime}$ couplings. Note that this amplitude cannot contribute 


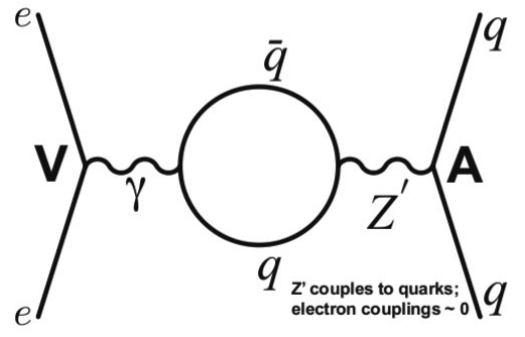

Fig. 75. A Feynman diagram for an amplitude with a vector electron current and axial-vector hadron current which would be sensitive to a heavy new vector boson that couples to quarks and has no couplings to leptons [321].

to any tree-level amplitudes nor amplitudes involving the $C_{1 i}$ couplings at the quantum loop level. The projected uncertainty from the JLab measurements will be sensitive to a lepto-phobic $Z^{\prime}$ with a mass $\lesssim 150 \mathrm{GeV}$, significantly better than the current limit from indirect searches when there is no significant $Z-Z^{\prime}$ mixing.

The JLab extraction will rely on a simultaneous fit of electroweak couplings, higher-twist effects and violation of charge symmetry to a series of $A_{P V}$ measurements in narrow $x$ and $Q^{2}$ bins. It is highly motivated to find ways to improve the sensitivity to the $C_{2 i}$ couplings further, given its unique sensitivity for $\mathrm{TeV}$-scale dynamics such as the aforementioned $Z^{\prime}$ bosons. The kinematical range for the $A_{P V}$ measurement at the EIC would enable a significantly improved statistical sensitivity in the extraction of the $C_{2 i}$ couplings. Apart from statistical reach, the EIC measurements will have the added advantage of being at significantly higher $Q^{2}$ so that higher-twist effects should be totally negligible.

A study of the statistical reach shows that an EIC measurement can match the statistical sensitivity of the $12 \mathrm{GeV}$ JLab measurement with $\sim 75 \mathrm{fb}^{-1}$. It is also worth noting that the EIC measurements will be statisticslimited, unlike the JLab measurement. The need for precision polarimetry, the limiting factor in fixed target measurements, will be significantly less important at the corresponding EIC measurement because $2 C_{2 u}-C_{2 d}$ would be extracted by studying the variation of $A_{P V}$ as a function of the fractional energy loss parameter, $y$. Thus, with an integrated luminosity of several $100 \mathrm{fb}^{-1}$ in Stage II of the EIC, the precision could be improved by a further factor of 2 to 3. Depending on the discoveries at the LHC over the next decade, it is quite possible that such sensitivity to $C_{2 i}$ couplings, which is quite unique, would prove to be critical to unravel the nature of $\mathrm{TeV}$-scale dynamics.

\subsection{EIC requirements for electroweak physics measurements}

For the CLFV $e \rightarrow \tau$ transition search, it was pointed out that the collider environment facilitates separating potential signal events from conventional DIS events, as demonstrated by successful searches carried out at modest integrated luminosity at HERA. This is because the lepton in the final state tends to be isolated at low $Q^{2}$ from the hadron jet. The detector will have to be suitably designed so as to allow high-energy electron identification at high $Q^{2}$ where it might be buried in the jet fragment.

In addition, compared to HERA, it is reasonable to expect that the EIC detector will have significant technological enhancements that will allow increased sensitivity, and improved background rejection. The momentum resolution for tracks and the granularity of the calorimeter will be improved. Detector coverage will extend down to much smaller angles. Most importantly, we envision a vertex detector that will greatly improve the robustness of the search. Since the lifetime of the $\tau$ lepton is $290 \mathrm{fs}$, for the typical energies expected for signal events, the decay length will be between a few $100 \mu \mathrm{m}$ and several mm, which will allow displaced vertices to be easily identified.

For the flavor-diagonal precision electroweak measurements, the apparatus being designed will be adequate to select the events required to make the precision asymmetry measurements. The challenge will be in controlling normalization errors, particularly the electron beam polarization. For the anticipated precision of the $A_{P V}$ measurements, the electron beam polarization must be monitored to significantly better than $1 \%$. At the completion of the JLab12 program, it is expected that techniques will be developed to monitor the beam polarization at the level of $0.5 \%$. It will be necessary to transfer this technology to the collider environment.

\section{The accelerator designs and challenges ${ }^{15}$}

\section{1 eRHIC $^{16}$}

eRHIC is a future Electron-Ion Collider (EIC) based on the existing Relativistic Heavy Ion Collider (RHIC) hadron facility with its two intersecting superconducting rings, each $3.8 \mathrm{~km}$ in circumference. The replacement cost of the RHIC facility is about two billion US dollars, and eRHIC will take full advantage of, and build on, this investment.

A polarized electron beam with an energy up to $21 \mathrm{GeV}$ would collide with a number of ion species accelerated in the existing RHIC accelerator complex, from polarized protons with a top energy of $250 \mathrm{GeV}$ to fullystripped uranium ions with energies up to $100 \mathrm{GeV} / \mathrm{u}$ covering a center-of-mass energy range from 30 to $145 \mathrm{GeV}$ for polarized $e+p$, and from 20 to $90 \mathrm{GeV}$ for $e+\mathrm{A}$ (for large $A$ ). Using the present significant margin of the RHIC superconducting magnets, the maximum beam energy could be increased by 10 or more percent.

The eRHIC design is based on using one of the two RHIC hadron rings and a multi-pass Energy Recovery Linac (ERL). Using an ERL as the electron accelerator assures high luminosity in the $10^{33}-10^{34} \mathrm{~cm}^{-2} \mathrm{~s}^{-1}$ range (fig. 76). Most of the electron accelerator components, including the injector, the ERL and the recirculation passes, are located inside the RHIC tunnel. eRHIC will be able to provide electron-hadron collisions in up to three interaction regions.

\footnotetext{
15 Conveners: Andrew Hutton and Thomas Roser.

16 See ref. [322] for an updated design of eRHIC.
} 


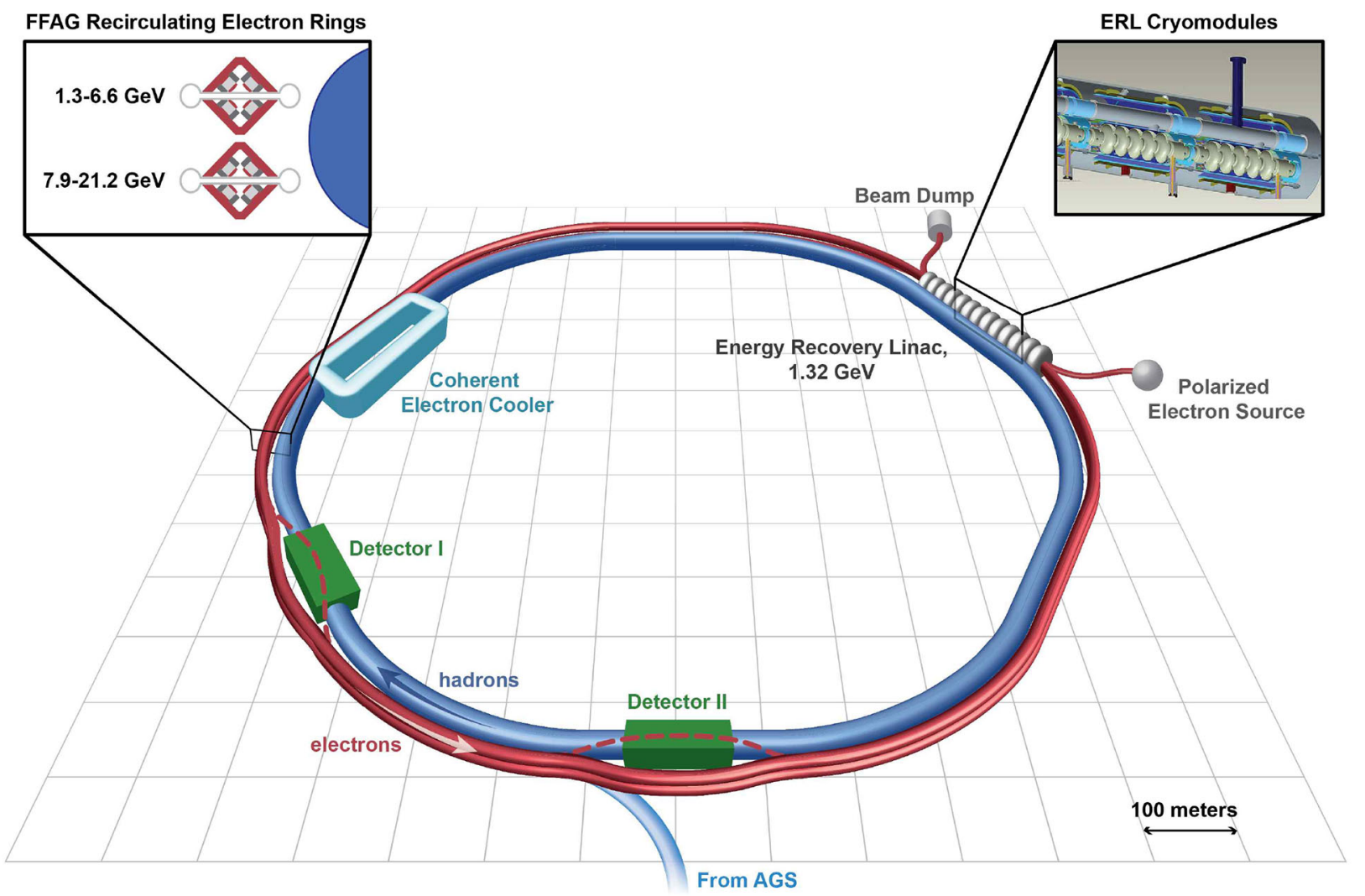

Fig. 76. The layout of the ERL-based, $21 \mathrm{GeV} \times 250 \mathrm{GeV}$ high-energy high-luminosity eRHIC.

Table 6. Projected eRHIC parameters and luminosities. The parameters in parentheses correspond to possible future upgrade, HL-eRHIC.

\begin{tabular}{|l|c|c|c|}
\hline & Electron & Proton & $\mathrm{Au}$ \\
\hline Max. beam energy $[\mathrm{GeV} / \mathrm{n}]$ & 15.9 & 250 & 100 \\
Bunch frequency $[\mathrm{MHz}]$ & 9.38 & 9.38 & 9.38 \\
Bunch intensity (nucleons/electrons) $\left[10^{11}\right]$ & 0.33 & $0.3(3)$ & $0.6(2.2)$ \\
Beam current [mA] & 50 & $42(420)$ & $33(120)$ \\
Polarization [\%] & 80 & 70 & \\
RMS bunch length $[\mathrm{mm}]$ & 4 & $50(84)$ & $50(84)$ \\
RMS norm. emittance $(e-p / e-\mathrm{Au})[\mu \mathrm{m}]$ & $32 / 58$ & 0.3 & 0.2 \\
$\beta^{*}[\mathrm{~cm}]$ & 5 & 5 & 5 \\
Luminosity $\left[10^{33} \mathrm{~cm}^{-2} \mathrm{~s}^{-1}\right]$ & & $1.7(13)$ & $1.7(5.2)$ \\
\hline
\end{tabular}

eRHIC employs a cost effective way to provide multiple electron beam recirlculations by using Fixed-Field Alternating Gradient optics with very high momentum acceptance. It allows for up to 16 recirculations in only two vertically stacked beamlines. Additional savings are expected from the use of low-cost permanent magnets.

To reach the required performance, eRHIC will employ several novel technologies such as a polarized electron gun delivering a current of $50 \mathrm{~mA}$, strong hadron beam cooling using Coherent electron Cooling (CeC), a high current multi-pass Energy Recovery Linac (ERL), and acceleration of polarized He-3 to high energy. BNL, in collaboration with JLab and MIT, is pursuing a vigorous $R \& D$ program to address these technical challenges. Projected performance values for eRHIC are shown in table 6 .

\subsection{1 eRHIC design}

The eRHIC design was guided by beam dynamics limitations experimentally observed at existing colliders such as beam-beam tune spread of less than 0.015 and accelerator technology limits such as the focusing required to reach $\beta^{*}=5 \mathrm{~cm}$ for hadron beams. The incoherent space charge tune spread is limited to about 0.035 to support an adequate beam lifetime. For practical and cost considerations, we limited the maximum electron beam power loss due to synchrotron radiation to about $12 \mathrm{MW}$, which corresponds to a $50 \mathrm{~mA}$ electron beam current at $16 \mathrm{GeV}$ and about $18 \mathrm{~mA}$ at $21 \mathrm{GeV}$. This means that the luminosity of eRHIC operating with $21 \mathrm{GeV}$ electrons will be about $35 \%$ of the luminosity at $16 \mathrm{GeV}$ or lower electron 

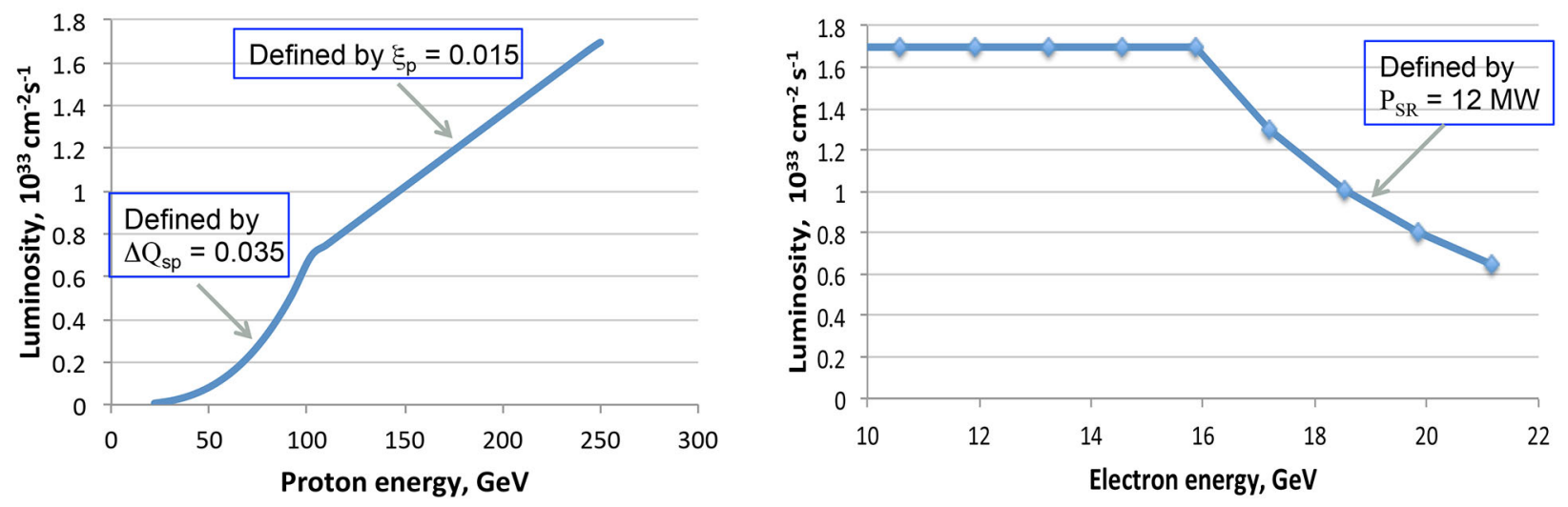

Fig. 77. eRHIC luminosity as a function of electron and proton beam energy. The left plot shows the luminosity dependence on proton energy at $E_{e}=15.9 \mathrm{GeV}$. The right plot presents the luminosity as a function of electron energy at $E_{p}=250 \mathrm{GeV}$.
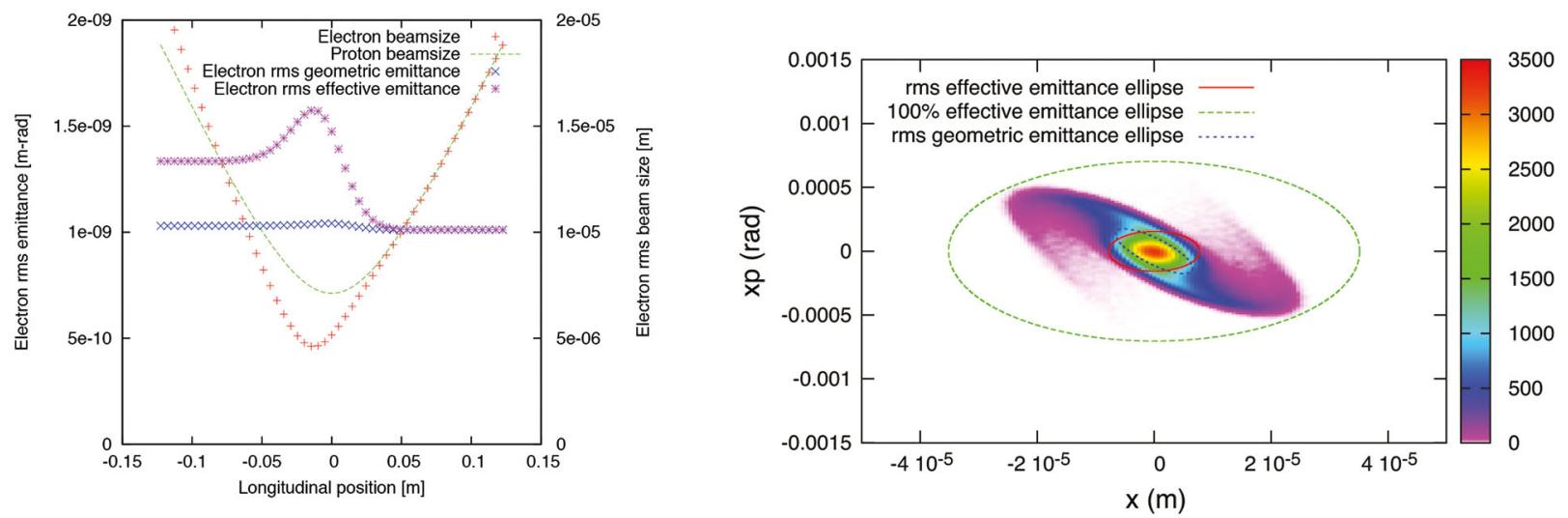

Fig. 78. The evolution of size and emittance of the e-beam during the collision (left) and distribution of electrons after collision with the hadron beam in eRHIC (right).

energy. The luminosity reachable with eRHIC is shown in fig. 77 as a function of electron and proton beam energy.

Since the ERL provides fresh electron bunches for every collision, the electron beam can be strongly distorted during the collision with the much stiffer hadron beam. This allows for greatly exceeding the beam-beam interaction limit that would apply for an electron beam in a storage ring. The electrons are strongly focused during the collision with the hadron beam (pinch effect), and the electron beam emittance grows by about $30 \%$ during the collision as shown in fig. 78. This increased beam emittance can still be easily accommodated by the beam transport during deceleration in the ERL. The only known effect of concern is the so-called kink instability. However, the ways of suppressing this instability within the range of parameters accessible by eRHIC are well understood. Small transverse and longitudinal beam emittances of the hadron beam in eRHIC are of critical importance, both for the attainment of high luminosity as well as for separating and detecting collision products scattered at small angles from the core of the hadron beam. For instance, the transverse emittance should be about ten times smaller than presently available in hadron machines. This requires a level of beam cooling that can only be achieved using Coherent electron Cooling ( $\mathrm{CeC}$ ), a novel form of beam cooling that promises to cool ion and proton beams by a factor of 10, both transversely and longitudinally, in less than 30 minutes. Traditional stochastic or electron cooling techniques could not satisfy this demand. $\mathrm{CeC}$ will be tested in a proof-of-principle experiment at RHIC by a collaboration of scientists from BNL, JLab, and TechX.

Unlike ring-ring colliders, the ERL allows for easy synchronization of the electron beam with the hadron beam in RHIC over a wide energy range from 20 to $250 \mathrm{GeV} / \mathrm{n}$ by using various sub-harmonics of the ERL RF frequency for the electron bunches, plus tuning a warm magnet delay line in a straight section of the hadron ring by up to $15 \mathrm{~cm}$. The ERL concept also allows for full electron beam polarization with longitudinal direction at the interaction point (IP) over the whole energy range. The electron polarization from the polarized electron gun, with the sign selectable for each bunch, is precessing in the horizontal plane during acceleration over multiple recirculation passes in the ERL. In order to preserve the polarization at $80 \%$ level special RF cavities operating at the 5 th harmonic of the main ERL cavities are applied to reduce 


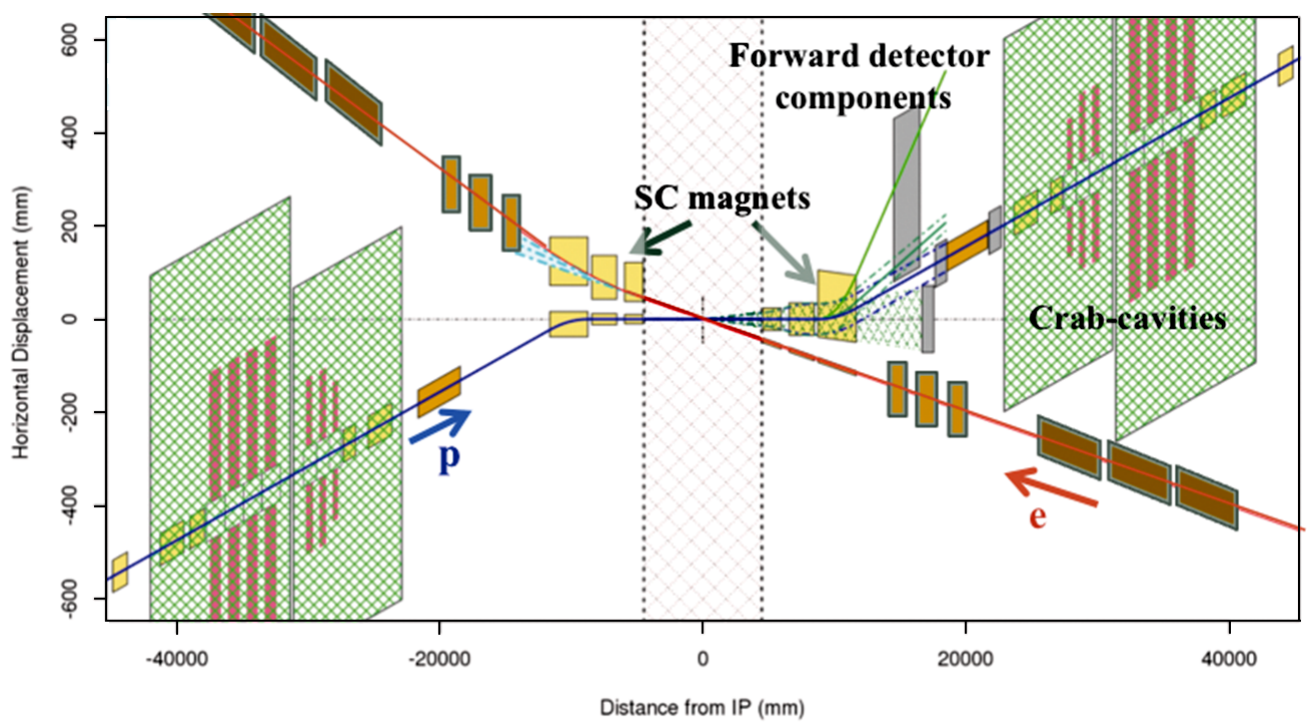

Fig. 79. The layout of the eRHIC interaction region.

beam energy spread and related spin decoherence. The choice of the ERL energy, $1.322 \mathrm{GeV}$, warranties the same polarization orientation at all experimental IPs.

The eRHIC hadron bunch intensity is significantly smaller than the one used during the present RHIC operation. It leaves a straightforward path for a future luminosity upgrade, HL-eRHIC, by increasing the proton intensity to $3 \cdot 10^{11} \mathrm{p} /$ bunch. Since the bunch length of the eRHIC hadron beam is small due to cooling, hadron ring upgrades will be required to allow for this intensity increase: copper coating of the beam pipe, an upgrade of the beam instrumentation and a new RF system. With these relatively modest upgrades the luminosity of the collider can be improved by one order of magnitude, exceeding $10^{34} \mathrm{~cm}^{-2} \mathrm{~s}^{-1}$ (table 6).

\subsection{2 eRHIC interaction region}

In the eRHIC IR design, the hadron and electron beam trajectories cross inside the detector at a $10 \mathrm{mrad}$ horizontal angle, as shown in fig. 79. The hadron beam is focused to $\beta^{*}=5 \mathrm{~cm}$ using both strong focusing by superconducting quadrupoles and an artificially excited $\beta$-function wave (ATS technique). The quadrupoles closest to the detector have a field gradient as high as $170 \mathrm{~T} / \mathrm{m}$, necessitating the application of superconducting magnet technology. Additional detector components are placed downstream of the hadron beam trajectory after a $16 \mathrm{mrad}$ bending magnet that separates the beam and collision products of interest. The chromaticity correction is arranged with arc sextupole families, taking advantage of the 90 degree lattice of the hadron ring arcs.

Head-on collisions of the electron and hadron bunches are restored with crab cavities located on either side of the interaction region. With a hadron ring lattice that provides large beta functions at the location of the crab cavities, an integral transverse RF field of $16 \mathrm{MV}$ on either side will provide the required $5 \mathrm{mrad}$ bunch rotation. The crab cavities for the electron ring are much more modest requiring only about $2 \mathrm{MV}$ transverse $\mathrm{RF}$ field.

The design of hadron superconducting magnets includes a free-field pass for the electron beam which is arranged for some magnets through the low-field area between the superconducting coils and for other magnets through their iron yoke. This configuration guarantees the absence of harmful high-energy X-ray synchrotron radiation in the vicinity of the detector. Furthermore, the electron beam is brought into the collision via a 130-meter long merging system, of which the last 60 meters use only soft bends with a magnet strengths of less than $10 \mathrm{mT}$ and less than $3 \mathrm{mT}$ for the final bend. Only $1.9 \mathrm{~W}$ of soft radiation from these magnets would propagate through the detector.

\section{1 .3 eRHIC R\&D}

R\&D for eRHIC is focusing on three main areas. To study the behavior of an ERL at very high beam intensity, an R\&D ERL that can accommodate up to $500 \mathrm{~mA}$ electron current is being assembled at BNL using a specially optimized 5-cell $704 \mathrm{MHz}$ SRF cavity with design features that are similar to cavities planned at the eRHIC ERL. The second project is the demonstration of Coherent electron Cooling (CeC) in RHIC using a $20 \mathrm{MeV}$ high brightness electron bunch to cool a $40 \mathrm{GeV} / \mathrm{n}$ gold bunch. Figure 80 shows a possible layout of $\mathrm{CeC}$ for RHIC. Finally, two efforts are underway to demonstrate the feasibility of producing a $50 \mathrm{~mA}$ polarized electron beam. One is based on a single large GaAs cathode and the other employes multiple GaAs cathodes that are used one at a time and the electron bunches are then combined with a rotating dipole field into a continuous electron beam. 


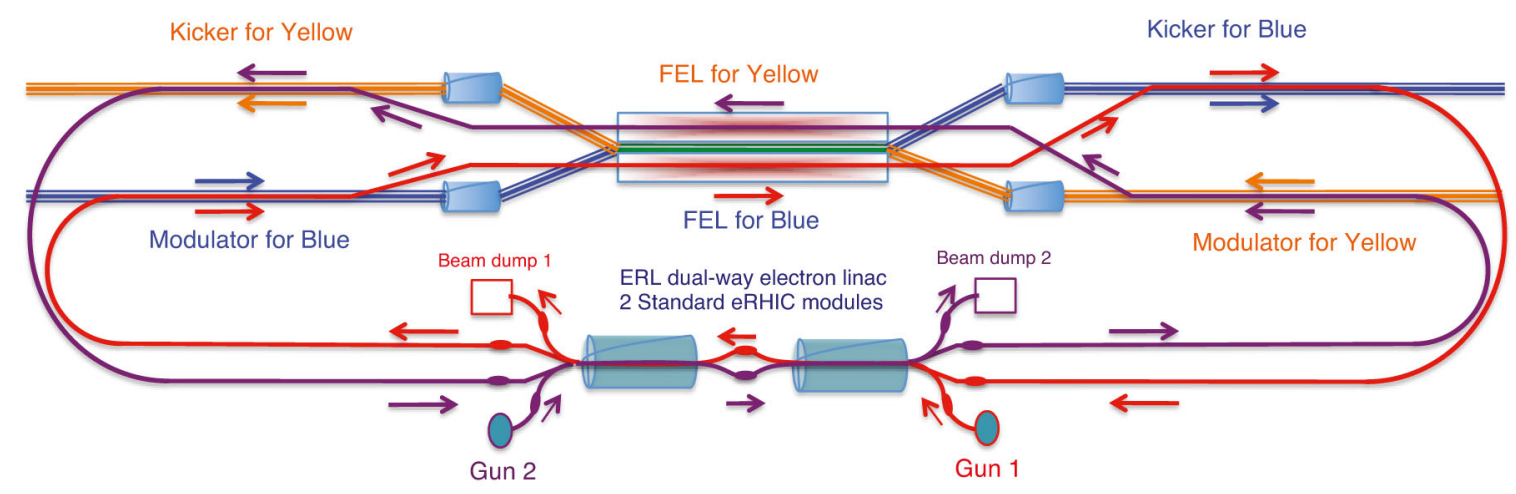

Fig. 80. A possible layout of RHIC CeC system cooling for both the yellow and blue beams.

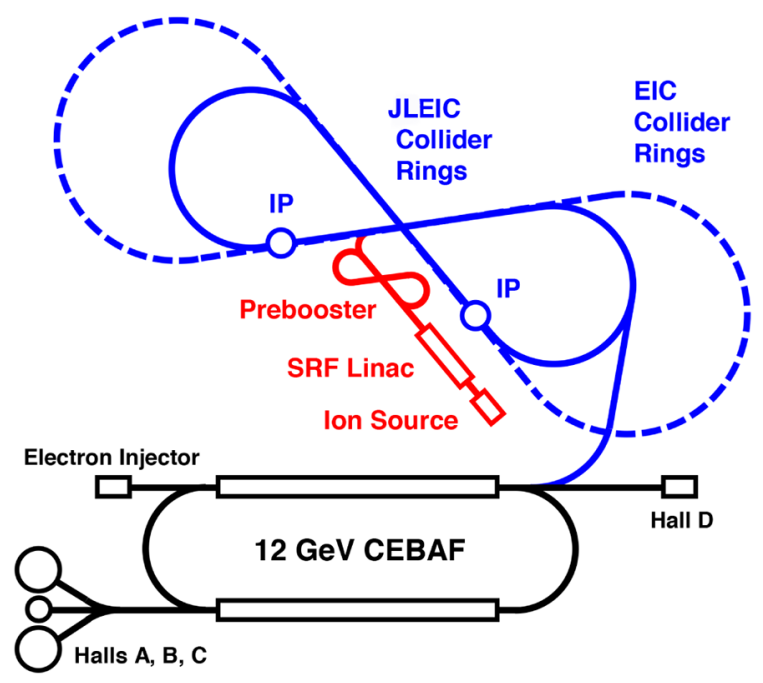

Fig. 81. Schematic layout of JLEIC.

\subsection{JLEIC $^{17}$}

\subsubsection{Jefferson Lab staged approach}

The JLab response to US user demand is to propose JLEIC [324] based on the $12 \mathrm{GeV}$ CEBAF recirculating SRF linac. This first stage of the JLab EIC program aims to cover a medium $\mathrm{CM}$ energy range up to $65 \mathrm{GeV}$ while meeting all other facility requirements. This approach achieves an optimized balance among the science program, technology R\&D and project cost. The JLEIC design maintains capability for future upgrades with maximum flexibility for changes in science goals and for costsaving facility equipment reuse. Presently, JLEIC is designed to collide 3 to $12 \mathrm{GeV}$ electrons with 25 to $100 \mathrm{GeV}$ protons or up to $40 \mathrm{GeV} / \mathrm{u}$ light to heavy ions, reach luminosities above $10^{34} \mathrm{~cm}^{-2} \mathrm{~s}^{-1}$ per interaction point (IP), and deliver $80 \%$ polarization for both electron and light ion beams. An envisioned upgrade would provide full coverage of the CM energy range up to $140 \mathrm{GeV}$ or above, and boost the peak luminosity close to $10^{35} \mathrm{~cm}^{-2} \mathrm{~s}^{-1}$ per IP.

JLEIC, designed as a traditional ring-ring collider and shown in fig. 81, takes advantage of several unique machine

\footnotetext{
17 See ref. [323] for a updated design of JLEIC.
}

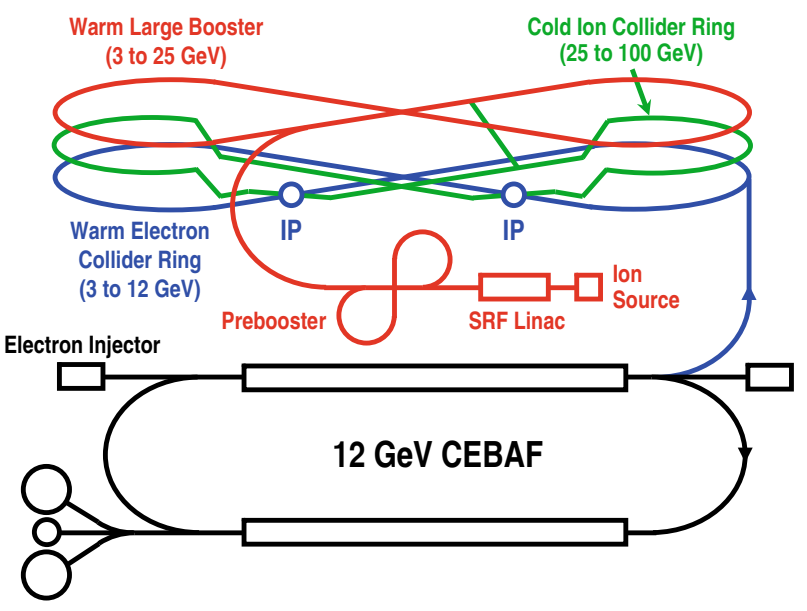

Fig. 82. An illustration of JLEIC, the large booster and collider rings are vertically stacked in a common tunnel.

design features for delivering high performance. It utilizes a high repetition rate $\mathrm{CW}$ electron beam from the CEBAF and matched ion beams from a new ion facility. This enables JLEIC to adopt a luminosity concept [325] which is based on high bunch repetition rate $\mathrm{CW}$ colliding beams and has been successfully proven in several lepton-lepton colliders for achieving an ultra high luminosity. A multiphased cooling scheme [326] provides strong cooling of ion beams not only at their formation stage but also during collisions. The JLEIC collider rings and ion boosters are in a figure- 8 shape which is a revolutionary solution [327] for preserving and controlling the beam polarization during acceleration and storage in a synchrotron. This design feature can deliver superior polarization of ion beams for experiments and is also the only practical way for accelerating and storing a medium or high energy polarized deuteron beam. Furthermore, the interaction regions are designed to provide ultra high to essentially full detector acceptance capability.

\subsubsection{Baseline design}

The two JLEIC collider rings are stacked vertically in a tunnel as illustrated in fig. 82. The electron ring stores 3 to $12 \mathrm{GeV}$ polarized electrons injected at full energy from 
Table 7. MEIC parameters at an example design point of particle energies for a full-acceptance detector (values for a highluminosity detector are given in parentheses).

\begin{tabular}{lccc}
\hline & & Proton & Electron \\
Beam energy & $\mathrm{GeV}$ & 60 & 5 \\
Collision frequency & $\mathrm{MHz}$ & \multicolumn{2}{c}{748.5} \\
Beam current/Particles per bunch & $\mathrm{A} / 10^{10}$ & $0.5 / 0.416$ & $3 / 2.5$ \\
Polarization & & $\sim 80 \%$ & $\sim 80 \%$ \\
RMS bunch length & $\mathrm{mm}$ & 10 & 7.5 \\
Normalized emit. $\left(\varepsilon_{x} / \varepsilon_{y}\right)$ & $\mu \mathrm{m}$ & $0.35 / 0.07$ & $53.5 / 10.7$ \\
Horizontal and vertical $\beta^{*}$ & $\mathrm{~cm}$ & $10 / 2(4 / 0.8)$ \\
Vertical beam-beam tune shift & & 0.015 & 0.03 \\
Laslett tune-shift & & 0.06 & $\mathrm{Small}$ \\
Detector space & $\mathrm{m}$ & $\pm 7(4.5)$ & \pm 3.5 \\
Luminosity per IP & $10^{33} \mathrm{~cm}^{-2} \mathrm{~s}^{-1}$ & \multicolumn{2}{c}{$5.6(14.2)$} \\
\hline
\end{tabular}

the CEBAF, while the SC ion collider ring stores 25 to $100 \mathrm{GeV}$ protons or fully stripped light to heavy ions with energies of the same magnetic rigidity. The ions execute a vertical excursion to the plane of the electron ring for collision at two IPs. An optional third detector may be placed at another IP. There is a third ring, the ion large booster with energy from 3 to $25 \mathrm{GeV}$ made of normal conducting magnets, in the same tunnel and stacked above the collider rings. The large figure- 8 shaped ring (the dashed line) in fig. 81 represents a future energy upgrade for reaching up to $20 \mathrm{GeV}$ electrons and $250 \mathrm{GeV}$ protons or $100 \mathrm{GeV} / \mathrm{u}$ ions.

Table 7 summarizes the JLEIC parameters at a design point of $5 \mathrm{GeV}$ electrons colliding with $60 \mathrm{GeV}$ protons [324]. The luminosity reaches $5.6 \times 10^{33} \mathrm{~cm}^{-2} \mathrm{~s}^{-1}$ for a full-acceptance detector. To reach such an acceptance, the machine-element-free detector space must be $7 \mathrm{~m}$ for ion beams on the downstream side; however, it can be shortened to $3.5 \mathrm{~m}$ on the upstream side. For the second detector which is optimized for higher luminosities while still maintaining a large detector acceptance, the detector space for ion beams can be reduced to $4.5 \mathrm{~m}$ so that the luminosity is increased to above $10^{34} \mathrm{~cm}^{-2} \mathrm{~s}^{-1}$.

JLEIC achieves high luminosities through a design choice characterized by a very high bunch repetition rate for both colliding beams [325]. It is about one to two orders of magnitude higher than that of the typical hadron colliders, however, it is similar to the bunch repetition rate of $e^{+} e^{-}$colliders. The bunch intensities of such beams are extremely small (usually by one to two orders of magnitude) even though the average current is several amperes. This opens up the possibility of very short bunch lengths for the ion beams, thus enabling a drastic reduction of the final focusing beta-star (to several $\mathrm{cm}$ or even less). As a result, the collider can reach a high luminosity.

Table 8 shows the luminosities of $e$-ion collisions for several different ion species.
To derive this parameter set, certain limits were imposed on several machine or beam parameters in order to improve robustness of the design and to reduce accelerator R\&D. These limits are largely based on previous experiences of lepton and hadron colliders and the present state of the art of accelerator technologies. They include

- ion SC magnet field is up to $6 \mathrm{~T}$;

- the stored beam currents are up to $0.5 \mathrm{~A}$ for protons or ions and $3 \mathrm{~A}$ for electrons;

- the electron synchrotron radiation power should not exceed $20 \mathrm{~kW} / \mathrm{m}$;

- maximum betatron function near an IP is $2.5 \mathrm{~km}$.

\subsubsection{Ion complex}

Figure 83 illustrates the schematic layout of the JLEIC ion complex. The ions from polarized or un-polarized sources are accelerated step-by-step to the colliding energies in the following major machine components: a $285 \mathrm{MeV}$ pulsed SRF linac, a $3 \mathrm{GeV}$ pre-booster synchrotron, a $25 \mathrm{GeV}$ large booster synchrotron, and finally a collider ring of 25 to $100 \mathrm{GeV}$.

Ion sources: The JLEIC ion sources will rely on existing technologies: an Atomic Beam Polarized Ion Source (ABPIS) [328] with Resonant Charge Exchange Ionization for producing polarized light ions $\mathrm{H}^{-} / \mathrm{D}^{+}$and ${ }^{3} \mathrm{He}^{++}$, and an Electron Beam Ion Source (EBIS) similar to the one currently in operation at BNL [329] for producing unpolarized light to heavy ions.

Ion linac: The technical design of a pulsed SRF ion linac [330], originally developed at Argonne National Laboratory as a heavy-ion driver linac for FRIB, has been adopted for the JLEIC proposal. This linac is very effective in accelerating a wide variety of ions from $\mathrm{H}^{-}$to ${ }^{208} \mathrm{~Pb}^{30+}$. 
Table 8. JLEIC luminosities for different ion species (values for a high-luminosity detector with a $4.5 \mathrm{~m}$ ion detector space are given in parentheses.)

\begin{tabular}{|l|c|c|c|c|c|c|c|c|}
\hline & & $e$ & $p$ & $d$ & ${ }^{3} \mathrm{He}^{++}$ & ${ }^{40} \mathrm{Ca}^{20+}$ & ${ }^{208} \mathrm{~Pb}^{82+}$ \\
\hline Energy & $\mathrm{GeV} / \mathrm{u}$ & 6 & 100 & 50 & 66.7 & 50 & 40 \\
\hline Current & $\mathrm{A}$ & 3 & \multicolumn{5}{|c|}{0.5} \\
\hline Particles per bunch & $10^{9}$ & 25 & 4.2 & 4.2 & 2.1 & 0.2 & 0.05 \\
\hline$\beta^{*}(x / y)$ & $\mathrm{cm}$ & $\begin{array}{c}1.6 \text { to } 2.8 \\
(0.61 \text { to } 1.1)\end{array}$ & \multicolumn{5}{|c|}{$6 / 2(2.4 / 0.8)$} \\
\hline $\begin{array}{l}\text { Beam-beam tune } \\
\text { shift (vertical) }\end{array}$ & $\begin{array}{c}0.023 \\
\text { to } 0.029\end{array}$ & 0.014 & 0.008 & 0.01 & 0.008 & 0.006 \\
\hline Luminosity/IP & $10^{34} \mathrm{~cm}^{-2} \mathrm{~s}^{-1}$ & & $\begin{array}{c}0.8 \\
(2.1)\end{array}$ & $\begin{array}{c}1.1 \\
(2.8)\end{array}$ & $\begin{array}{c}1.1 \\
(3.7)\end{array}$ & $\begin{array}{c}1.1 \\
(2.8)\end{array}$ & $\begin{array}{c}1.1 \\
(2.8)\end{array}$ \\
\hline
\end{tabular}

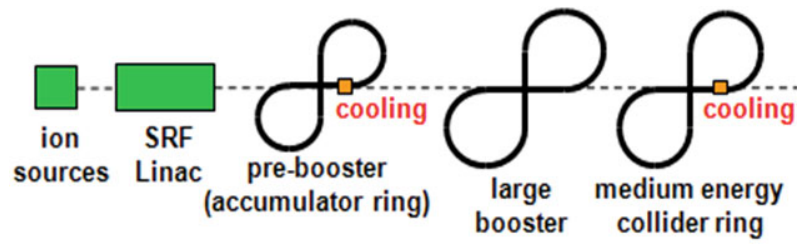

Fig. 83. Schematic layout of the ion complex.

Pre-booster/accumulator ring: The pre-booster synchrotron accepts linac pulses of any ion species, after accumulation and acceleration, transfers them to the large booster for further acceleration. It utilizes either the painting technique for $\mathrm{H}^{-} / \mathrm{D}^{-}$or the $\mathrm{DC}$ electron cooling for lead or other heavy ions during multi-turn injections from the linac.

Large booster: This booster synchrotron is responsible for accelerating protons to $25 \mathrm{GeV}$ or ions to $12.5 \mathrm{GeV} / \mathrm{u}$ before transporting them to the collider ring. Its circumference is four times that of the pre-booster.

A key design requirement for both booster synchrotrons is sufficiently high transition gamma such that the ions never cross the transition energy during acceleration in order to prevent particle loss associated with such a crossing.

\subsubsection{Collider rings}

The two JLEIC collider rings have nearly identical footprints with a circumference of approximately $1470 \mathrm{~m}$. The figure- 8 crossing angle is $60^{\circ}$. The two rings intersect at two symmetric points in two long straights for mediumenergy collisions. A third crossing point can be arranged for an extra detector. The long straights also accommodate necessary utility components such as injection, ejection, RF systems and electron cooling. One universal spin rotator consisting of two SC solenoids and two sets of arc dipoles is placed at each end of the two electron arcs. In the ion collider ring, a transition from a low bunch frequency to $750 \mathrm{MHz}$ repetition rate takes place.

\subsubsection{Interaction regions}

The primary detector of JLEIC is unique in its ability to provide essentially full acceptance to all fragments from collisions. The interaction region (IR) design [331] is optimized to support this detector acceptance. It relies on several features including a relatively large $50 \mathrm{mrad}$ crab crossing angle, large-aperture final focusing (FF) quadrupoles and spectrometer dipoles as well as a large $(7 \mathrm{~m})$ machine-element-free detection space downstream of the ion beam.

The large crab crossing angle of the JLEIC design not only allows quick separation of the two colliding beams near an IP for avoiding parasitic collisions and makes sufficient space for placement of IR magnets but also moves the spot of poor resolution along the solenoid axis into the periphery and minimizes the shadow of the electron FF quadrupoles. Crab cavities will be utilized for restoring head-on collisions.

The IR design takes special care to minimize radiation in the detectors and maintain good background. Bending of electrons in the straight sections is reduced to the minimum, thus the ions are arranged to travel to the plane of the electron ring for collisions. The electron beam line is parallel to the detector solenoid axis for avoiding extra bending by the solenoid. The detectors are placed far from the electron arc exits to minimize the synchrotron radiation background and close to the ion arc exits to minimize the hadronic background due to the ion beam scattering on the residual gas.

Figure 84 shows the layout of an IR. The end section of the ion arc upstream of an IP is shaped to produce a net $50 \mathrm{mrad}$ horizontal angle between the ion and electron beams while the ion beam line segment downstream of the IP is designed to make a $2 \mathrm{~m}$ transverse separation between the ion and electron beams.

Due to kinematic considerations, more detector space is needed downstream of the IP than upstream along the ion beam direction. Consequently, the upstream ion final focusing block (FFB) is placed closer to the IP (at a distance of $3.5 \mathrm{~m}$ ) than the downstream one (at a distance of $7 \mathrm{~m}$ ), yielding an asymmetric detector region. Each ion FFB is a quadrupole triplet allowing for a more 


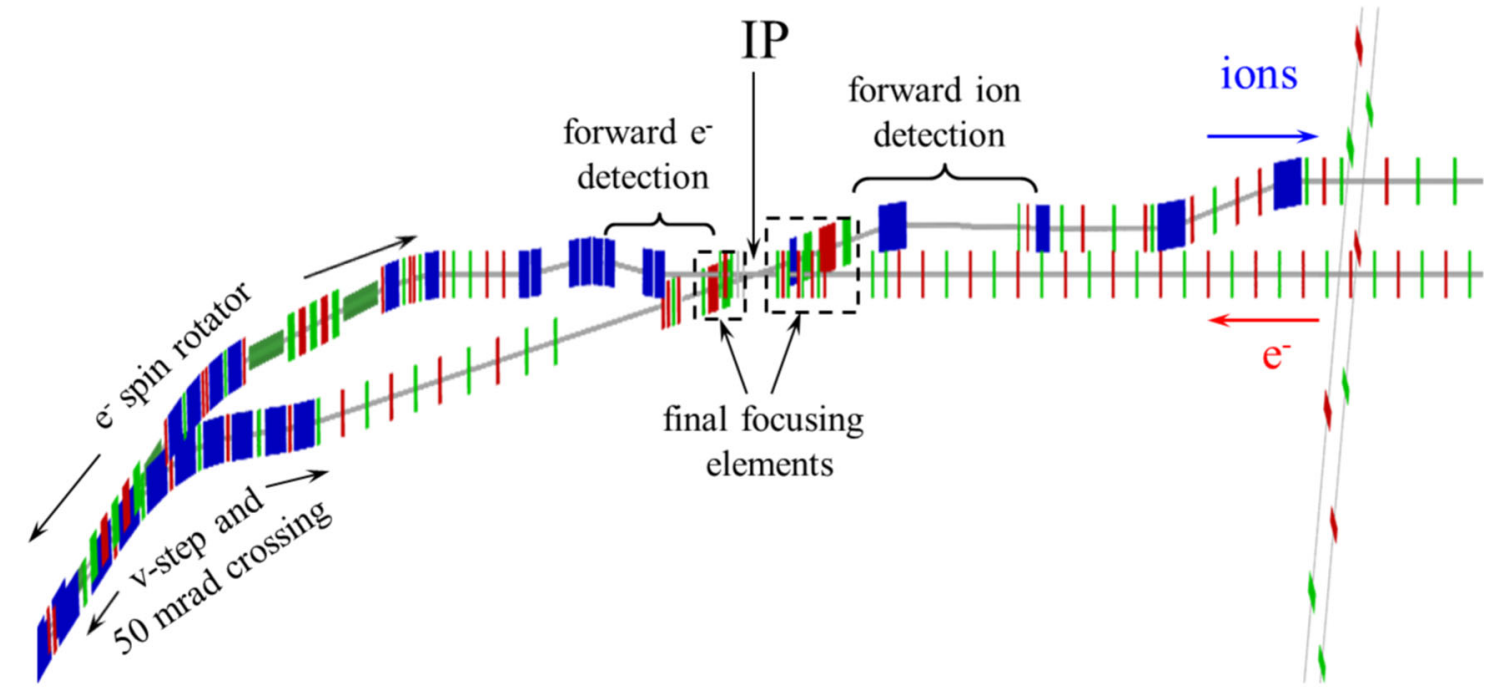

Fig. 84. The layout of the interaction region associated with a full acceptance detector.

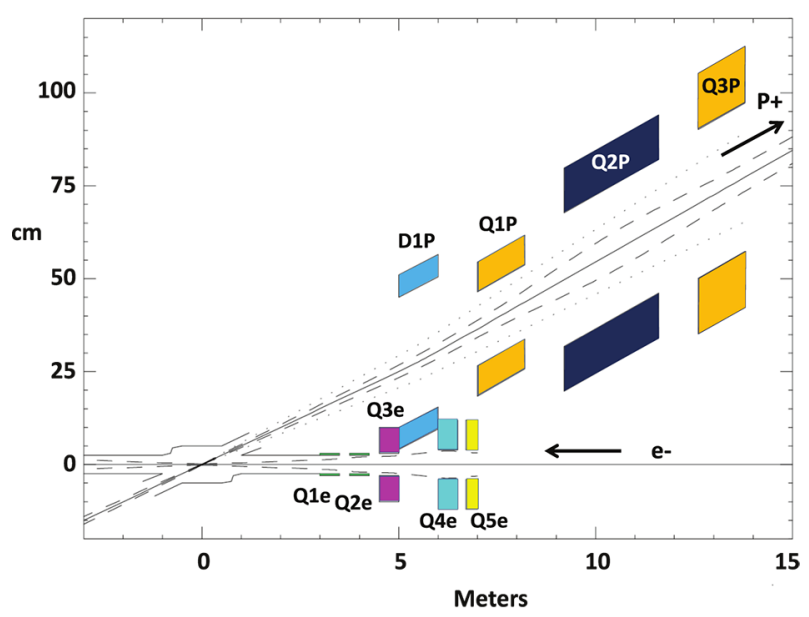

Fig. 85. Layout of the IR in the forward ion direction.

flexible control of the beta functions. Electron FFBs are also based on quadrupole triplets but include additional permanent-magnet quadrupoles placed at the front of the FFBs. The permanent-magnet quadrupoles have a small size and can be placed closer to the IP. Change of their focusing strength with energy is compensated by adjusting the regular electromagnetic FFB quadrupoles. The electron FFBs are placed $3 \mathrm{~m}$ away from the IP. The downstream ion and electron FF quadrupoles are designed with large apertures for forward detection and are followed by spectrometer dipoles. Additionally, there is a weak spectrometer dipole in front of the downstream ion FFB. Such a design shown in fig. 85 satisfies the detector requirements while minimizing the chromatic contribution of both the ion and electron FFBs.

Sufficient machine-element-free space is reserved beyond the downstream FFBs and spectrometer dipoles for detection purposes. Both the ion and electron beams are focused again towards the end of this element-free space to allow closer placement of the detectors, which, in combi- nation with relatively large dispersion at those points, enhances the forward detector's momentum resolution. The dispersion generated by the spectrometer dipoles is suppressed on the ion side by a specially designed section, which also controls the beam line geometry, while on the electron side the dispersion suppression is done by a simple dipole chicane whose parameters are chosen to avoid a significant impact on the electron equilibrium emittance.

Due to the strong beam focusing at the IPs, the chromatic effect of the FFBs in both the ion and electron collider rings is very significant and requires proper compensation. JLEIC employs a local compensation approach where dedicated chromaticity compensation blocks cancel the chromatic kick of the FFBs. Initial simulations using this concept yielded encouraging results [332]. Detailed studies and optimization of the non-linear dynamics are underway.

\subsubsection{Ion polarization}

The JLEIC is designed to preserve and control high polarization of proton, light ion (deuteron, helium-3 and possibly lithium), and electron beams as required by the nuclear physics program.

A figure-8 shape is adopted for all ion booster synchrotrons and both collider rings to preserve and control beam polarization during acceleration and storage. The complete cancellation of the spin procession in the two halves of a figure- 8 ring leads to an energy-independent zero spin tune and the lack of a preferred periodic spin direction so that the polarization can be effectively controlled by small magnetic fields. In particular, a figure- 8 design is the only practical way for accelerating polarized medium energy deuterons due to their small anomalous magnetic moment. The polarization can be stabilized by weak solenoid fields lower than $3 \mathrm{Tm}$ in all ion rings with polarization directions matched at the beam injection and extraction locations. In the ion collider ring, either longitudinal or transverse polarization can be obtained at IPs 


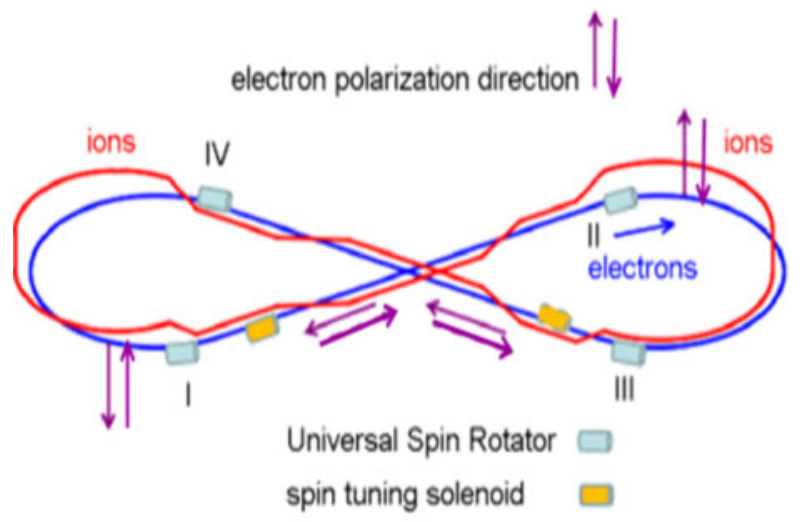

Fig. 86. Polarization configuration in the JLEIC electron collider ring.

using weak radial-field dipoles $(<0.25 \mathrm{~T} \mathrm{~m} \mathrm{each)} \mathrm{for} \mathrm{pro-}$ tons or weak solenoids $(<1.5 \mathrm{Tm}$ each) for deuterons. The required spin flipping can be implemented by changing the source polarization, manipulating the polarization direction in the collider ring using weak fields, or using RF magnetic fields to flip the polarization of a stored beam. A polarization of up to $85 \%$ for ion beams is expected [333].

In the ion pre-booster and large booster, one small solenoid placed in a straight section is sufficient to attain longitudinal polarization for both deuterons and protons. The maximum integral of the longitudinal solenoid field is about $0.3 \mathrm{Tm}$ for deuterons and $1.5 \mathrm{Tm}$ for protons. The spin tune induced by the solenoid field is much greater than the strength of the zero-harmonic spin resonance.

In the collider ring, deuteron polarization can be efficiently controlled by small solenoids. A symmetric scheme has been developed for deuteron polarization control with two solenoids on both sides of the experimental straight. The maximum field integral in a single solenoid at the maximum energy does not exceed $1.5 \mathrm{~T} \mathrm{~m}$. The proton polarization in the collider ring can be controlled using the schemes for deuterons as well. However, at higher proton energies, it is more efficient to use radial fields that can be significantly lower than the longitudinal fields.

\subsubsection{Electron polarization}

A highly polarized electron beam is injected from the CEBAF into the electron collider ring at full energy. As shown in fig. 86, the electron polarization is designed to be vertical in the arcs to minimize spin diffusion (i.e. depolarization) and longitudinal at IPs for experiments. This is achieved by means of a universal spin rotator [334] illustrated in fig. 87. Four such spin rotators, located at the ends of the two arcs, rotate the polarization in the whole energy range, leaving the design orbit intact.

Desired spin flipping can be attained by alternating the helicity of the photo-injector driver laser to provide two long opposite polarization bunch trains. The polarization configuration is chosen to have the same polarization direction (either up or down) in the two arcs by setting opposite solenoid fields in the two spin rotators at the both ends of the same experimental straight [335].

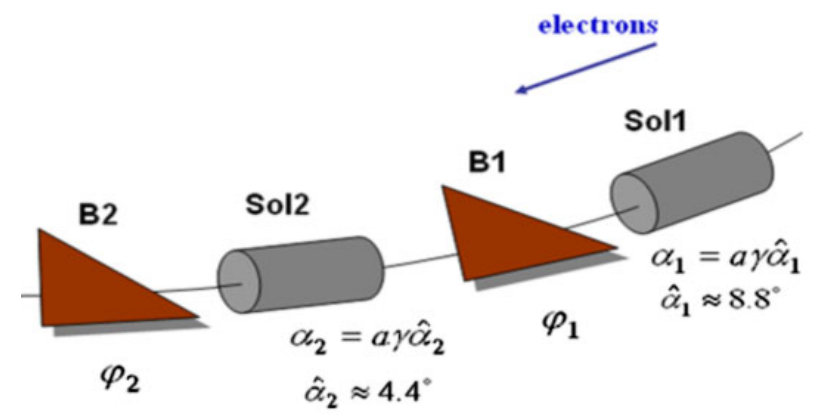

Fig. 87. Schematic drawing of a universal spin rotator consisting of two sets of arc dipoles (B1 and B2) and two solenoids (Sol1 and Sol2).

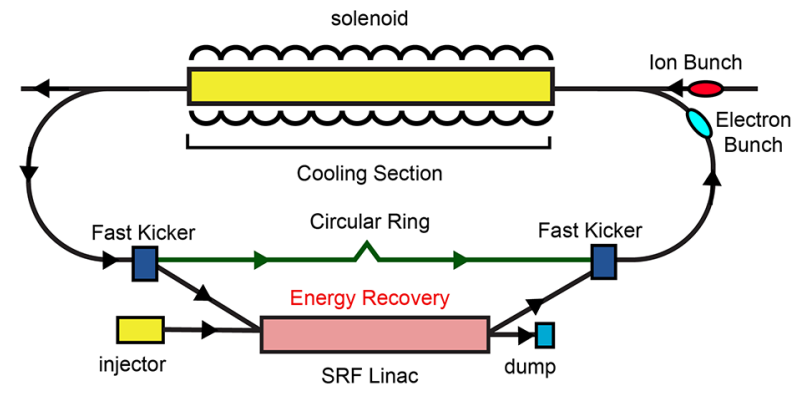

Fig. 88. Schematic of electron cooling for the JLEIC.

Such a configuration, with a figure- 8 shape, removes the spin tune energy dependence, therefore significantly reducing the quantum depolarization. The spin tune can be easily controlled by weak solenoid(s) in the experimental straights, where the polarization is along the longitudinal direction.

The polarization lifetime is estimated to be reasonably long (a few hours) at low energies however it drops to tens to a few minutes at higher energies $(9 \mathrm{GeV}$ and above). To obtain a high polarization in the whole energy range, continuous injection (top-off) of highly polarized electrons from the CEBAF is used to assist preservation of the stored beam's polarization, especially at higher energies. An equilibrium polarization of up to $80 \%$ in the whole energy range can be achieved [335].

\subsubsection{Electron cooling}

Cooling of ion beams is essential to achieve high luminosities over a broad CM energy range in JLEIC. The design relies on the traditional electron cooling method and adopts a concept of multi-phase cooling of bunched ion beams of medium energies. Electron cooling is first utilized for assisting accumulation of ions in the pre-booster. It then provides initial cooling at the ejection energy of the pre-booster, taking advantage of high cooling efficiency at low energies. In the ion collider ring, electron cooling is used at the injection energy and also after acceleration to the collision energy. Most importantly, electron cooling will be operated continuously during collisions to suppress IBS-induced beam emittance growth. Shortening of the bunch length $(1 \mathrm{~cm}$ or less) that results from electron 

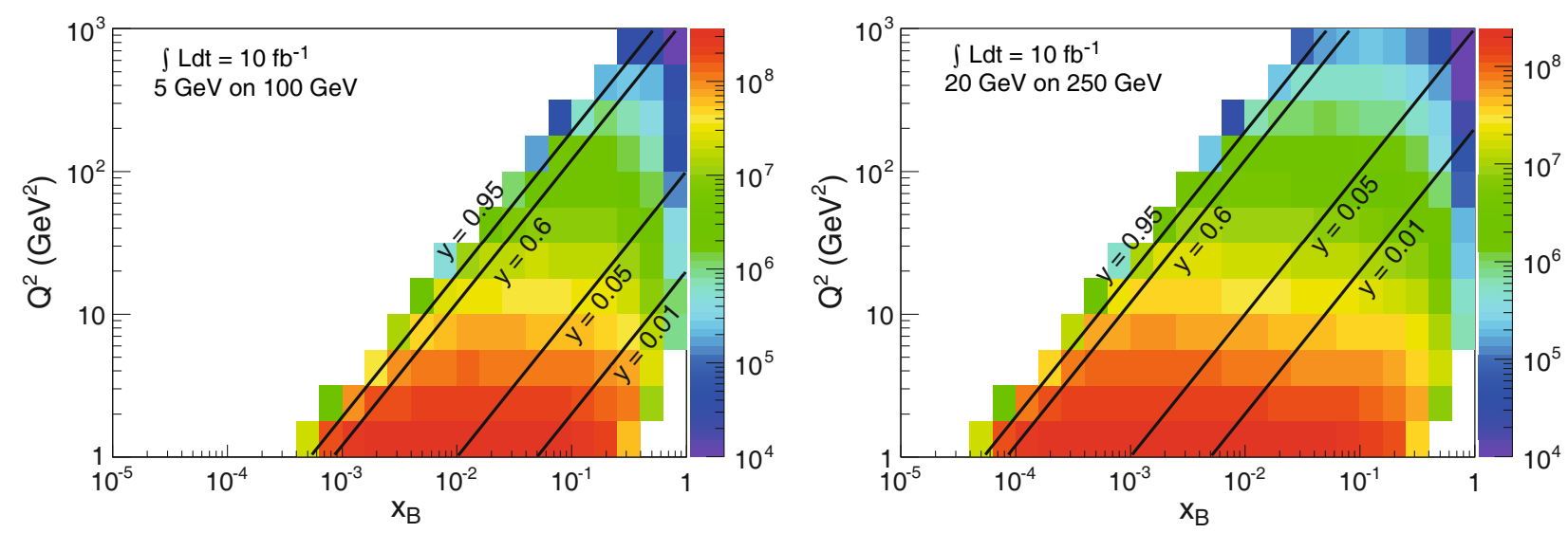

Fig. 89. The $x-Q^{2}$ plane for center-of-mass energy $45 \mathrm{GeV}$ (left) and $140 \mathrm{GeV}$ (right). The black lines indicate different $y$-cuts placed on the scattered lepton kinematics.

cooling of the ion beam captured in a high-voltage SRF field is critical for high luminosity in the JLEIC since it facilitates the strong focusing of the colliding beams and also implementation of crab crossing at the IPs for achieving an ultra-high bunch collision rate.

Two electron coolers are required to implement the JLEIC cooling scheme. In the pre-booster, a DC cooler with an up to $2 \mathrm{MeV}$ electron beam energy is needed and is within the state-of-art. In the ion collider ring, an energyrecovery-linac-based electron cooler [326] illustrated by a schematic drawing in fig. 88 will be responsible for cooling the medium-energy ions. Two accelerator technologies - an ERL and a circulator ring - play critical roles in the success of this facility by providing perfect solutions to the two challenging aspects of the facility: the high current and high power of the cooling electron beam. For example, a $1.5 \mathrm{~A} 50 \mathrm{MeV}$ cooling beam ( $75 \mathrm{MW}$ of power) can effectively be provided by a $15 \mathrm{~mA}(30 \mathrm{~kW}$ of active beam power) from the injector/ERL if the cooling beam makes 100 turns in the circulator cooler ring.

The JLEIC will reach its ultimate full luminosity at the $10^{34} \mathrm{~cm}^{-2} \mathrm{~s}^{-1}$ scale with envisioned electron cooling scheme utilizing a circulator cooling ring. Nonetheless, to reduce any dependence on this scheme, the JLEIC electron cooling can be implemented in various stages. Utilizing DC cooling at pre-booster energies - with similar requirements as the established DC cooling at FNAL [336] and FZ-Jüelich [337] — will already allow a peak luminosity above $3 \times 10^{33} \mathrm{~cm}^{-2} \mathrm{~s}^{-1}$ if only projecting a single-turn ERL cooler without a circulator ring.

\section{The EIC detector requirements and design ideas $^{18}$}

\subsection{Introduction}

The physics program of an EIC imposes several challenges on the design of a detector, and more globally the extended interaction region, as it spans a wide range in

\footnotetext{
18 Conveners: Elke C. Aschenauer and Tanja Horn.
}

the center-of-mass energy, different combinations of both beam energy and particle species, and several distinct physics processes. The various physics processes encompass inclusive measurements $\left(e p / \mathrm{A} \rightarrow e^{\prime}+X\right)$, which require the detection of the scattered lepton and/or the hadrons of the full scattered hadronic debris for which $E-p_{z}^{h a d}$ is different from zero; semi-inclusive processes $\left(e p / \mathrm{A} \rightarrow e^{\prime}+h+X\right)$, which require detection in coincidence with the scattered lepton of at least one (current or target region) hadron; and exclusive processes $\left(e p / \mathrm{A} \rightarrow e^{\prime}+N^{\prime} / \mathrm{A}^{\prime}+\gamma / m\right)$, which require the detection of all particles in the reaction with high precision. The figures in sect. 6.2 demonstrate the differences in particle kinematics of some representative examples of these reaction types, as well as differing beam energy combinations. The directions of the beams are defined as for HERA at DESY: the hadron beam is in the positive $z$-direction $\left(0^{\circ}\right)$ and the lepton beam is in the negative $z$-direction $\left(180^{\circ}\right)$.

\subsection{Kinematic coverage}

\section{2 .1 y coverage}

Figure 89 shows the $x-Q^{2}$ plane for two different center-ofmass energies. In general, the correlation between $x$ and $Q^{2}$ for a collider environment is weaker than for fixedtarget experiments. However, an important consideration is the extreme range of values of the inelasticity $y$. At large $y$, radiative corrections become large, as illustrated in fig. 7.25 in ref. [2]. There are two ways to address this: one is to calculate radiative corrections and correct for them; the other is utilize the hadronic activity in the detector together with cuts on the invariant mass of the hadronic final state.

The $x-Q^{2}$ correlations become stronger for small scattering angles or correspondingly small inelasticity. Here, radiative corrections are small, but the momentum and scattering angle resolution for the scattered lepton deteriorates. This problem is addressed by reconstructing the lepton kinematics from the hadronic final state using the Jacquet-Blondel method [338, 339]. At HERA, this method was successfully used down to $y$ of 0.005 . 


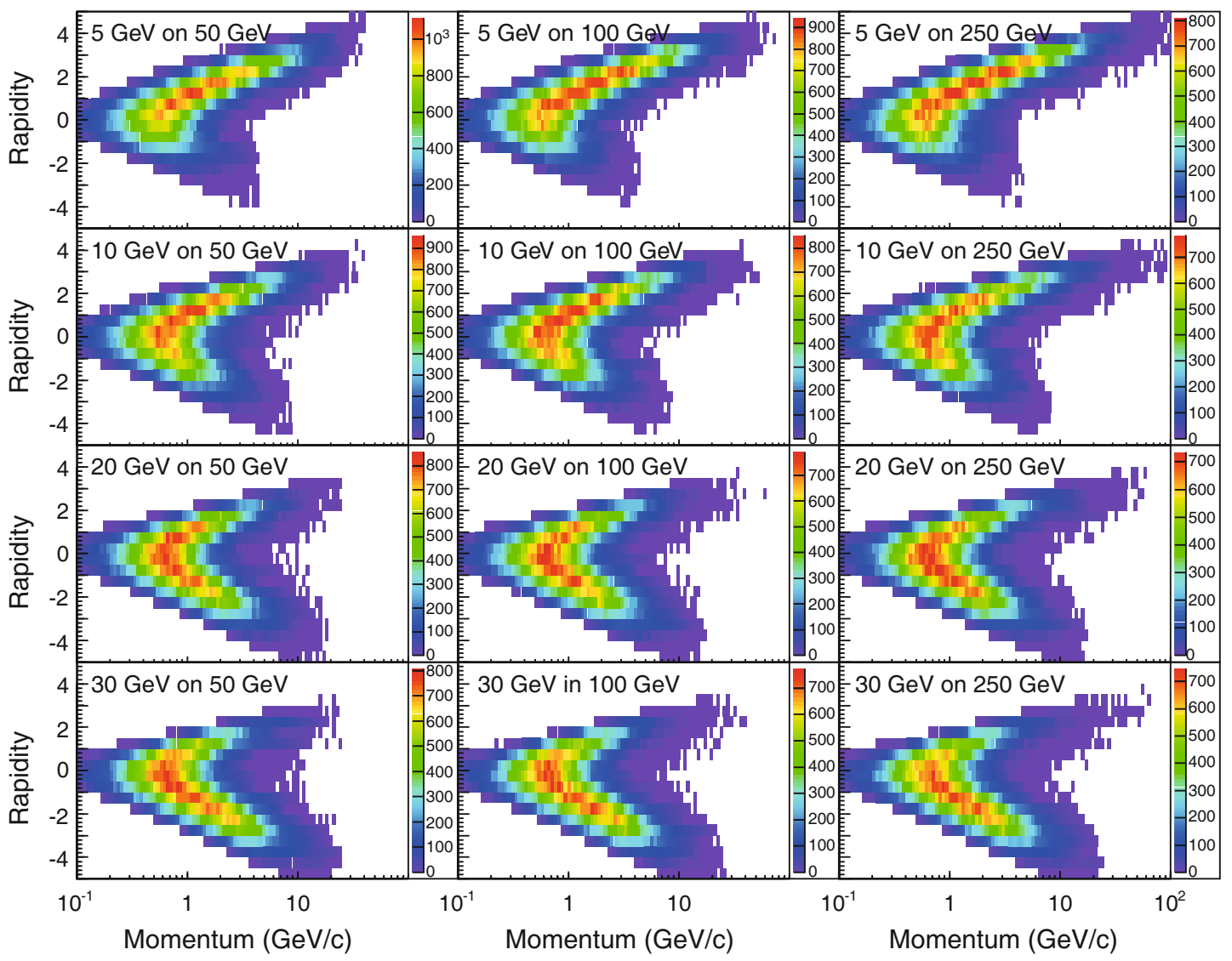

Fig. 90. Momentum vs. rapidity in the laboratory frame for pions from non-exclusive reactions. The following cuts have been applied: $Q^{2}>1 \mathrm{GeV}^{2}, 0.01<y<0.95,0.1<z$ and $-5<$ rapidity $<5$.

The main reason this hadronic method renders better resolution at low $y$ follows from the equation $y_{J B}=$ $E-P_{z}^{h a d} / 2 E_{e}$, where $E-P_{z}^{h a d}$ is the sum over the energy minus the longitudinal momentum of all hadronic final-state particles and $E_{e}$ is the electron beam energy. This quantity has no degradation of resolution for $y<0.1$ as compared to the electron method, where $y_{e}=1-\left(1-\cos \theta_{e}\right) E_{e}^{\prime} / 2 E_{e}$.

Typically, one can obtain for a given center-of-mass energy squared, roughly a decade of $Q^{2}$ reach at fixed $x$ when using only an electron method to determine lepton kinematics, and roughly two decades when including the hadronic method. If only using the electron method, one can increase the range in accessible $Q^{2}$ by lowering the center-of-mass energy, as can be seen from comparing the two panels of fig. 89. This is relevant for some semiinclusive and exclusive processes. The coverage of each setting is given by the product of $y \times s$. With a low $y_{\text {min }}$ cut, one thus needs fewer settings in $s$. However, this is an important consideration for any measurement, which needs to separate the cross-section components due to longitudinal and transverse photon polarization, i.e. the measurement of $F_{L}$ where one needs to have full $y$-coverage at all energies. The advantages and disadvantages of this solution are discussed in the two machine-specific detector sections of sect. 6 .

\subsubsection{Angle and momentum distributions}

Figure 90 shows the momentum versus rapidity distributions in the laboratory frame for pions originating from semi-inclusive reactions for different lepton and proton beam energy combinations. For lower lepton energies, pions are scattered more in the forward (ion) direction. With increasing lepton beam energy, the hadrons increasingly populate the central region of the detector. At the highest lepton energies, hadrons are even largely produced going backward (i.e. in the lepton beam direction). The kinematic distributions for kaons and additional protons/antiprotons are essentially identical to those of the pions. The distributions for semi-inclusive events in electron-nucleus collisions may be slightly altered due to nuclear modification effects, but the global features will remain.

Figure 90 also indicates the momentum range of pions in the central detector region $(-1<$ rapidity $<1)$ of typically $0.3 \mathrm{GeV} / c$ to $4 \mathrm{GeV} / c$ with a maximum of about $10 \mathrm{GeV} / c$. A combination of high-resolution time-of-flight (ToF) detectors (with timing resolutions $\delta t \sim 10 \mathrm{ps}$ ), a DIRC or a proximity focusing Aerogel RICH may be considered for particle identification in this region. Hadrons with higher momenta go typically in the forward (ion) direction for low lepton beam energies, and in the backward direction for higher lepton beam energies. The most 

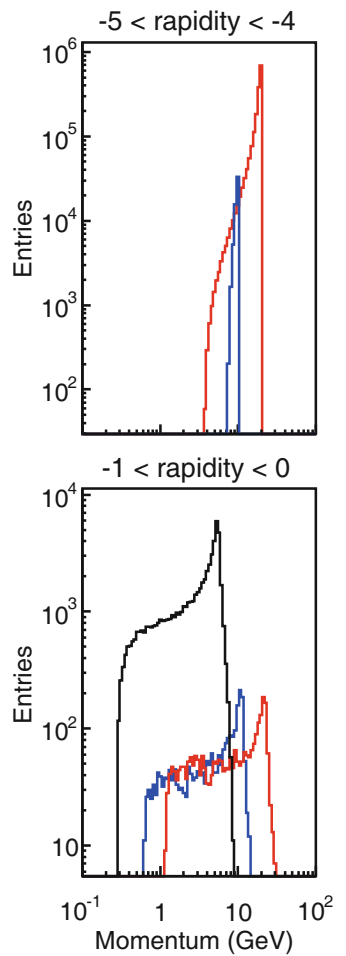
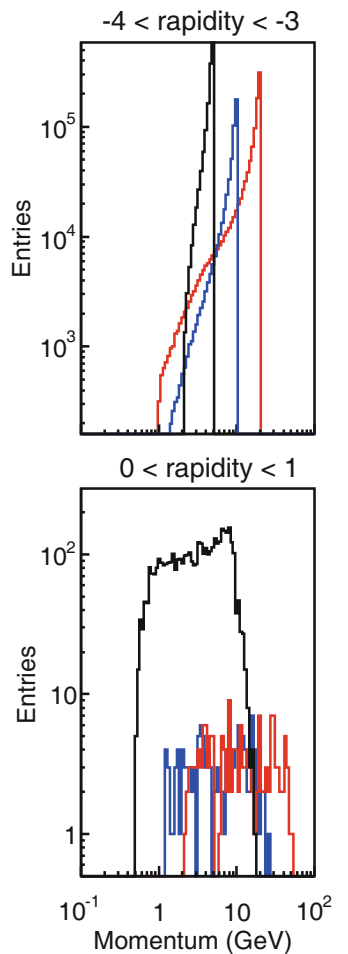
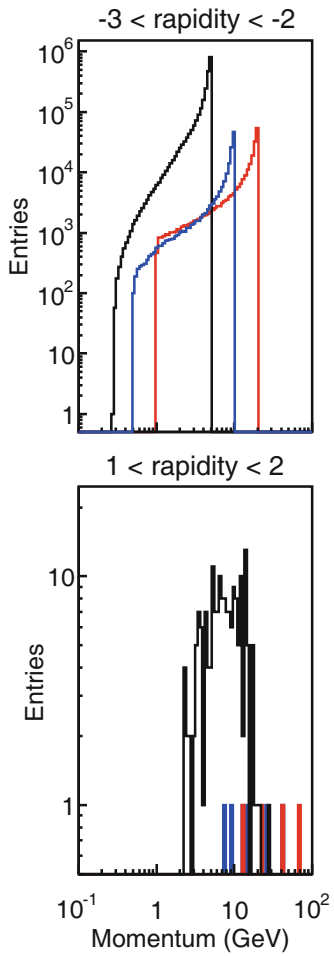

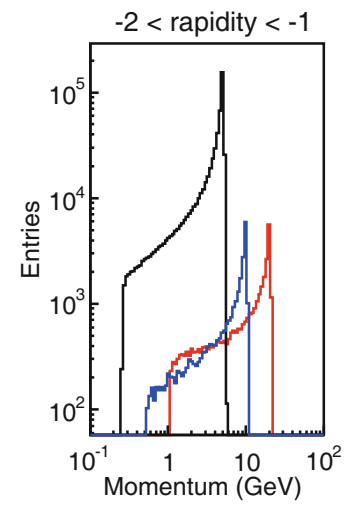

$20 \mathrm{GeV}$ on $250 \mathrm{GeV}$

$10 \mathrm{GeV}$ on $100 \mathrm{GeV}$

$5 \mathrm{GeV}$ on $50 \mathrm{GeV}$

Fig. 91. The momentum distribution for the scattered lepton for different center-of-mass energies and different rapidity bins in the laboratory frame. The following cuts have been applied: $Q^{2}>0.1 \mathrm{GeV}^{2}, 0.01<y<0.95$ and $-5<$ rapidity $<5$.

viable detector technology for this region of the detector is a Ring-Imaging Cherenkov (RICH) detector with dualradiators.

Figure 91 shows the momentum distribution for the scattered lepton for different rapidity bins and three different lepton-proton beam energy combinations. The $Q^{2}<$ $10 \mathrm{GeV}^{2}$ events typically correspond to negative rapidities $(\eta<-3)$ and $Q^{2}>10 \mathrm{GeV}^{2}$ correspond to rapidities $\eta>-2$ for $5 \mathrm{GeV} \times 50 \mathrm{GeV}$ and $\eta>-330 \mathrm{GeV} \times 50 \mathrm{GeV}$. Depending on the center-of-mass energy the rapidity distributions for hadrons (both charged and neutral) and the scattered lepton overlap and need to be disentangled. The kinematic region in rapidity over which hadrons and photons need to be suppressed with respect to electrons depends on the center-of-mass energy. For lower centerof-mass energies, electron, photon and charged hadron rates are roughly comparable at $1 \mathrm{GeV} / c$ total momentum and rapidity $=-3$. For the higher center-of-mass energy, electron rates are a factor of 10-100 smaller than photon and charged hadron rates, and comparable again at a $10 \mathrm{GeV} / c$ total momentum (see fig. 7.18 in ref. [2]). This adds another requirement to the detector: good electron identification. The kinematic region in rapidity over which hadrons and also photons need to be suppressed, typically by a factor of $10-100$, shifts to more negative rapidity with increasing center-of-mass energy.

Measuring the ratio of the energy and momentum of the scattered lepton, typically gives a reduction factor of $\sim 100$ for hadrons. This requires the availability of both tracking detectors (to determine momentum) and electromagnetic calorimetry (to determine energy) over the same rapidity coverage. By combining information from these two detectors, one also immediately suppresses the misidentification of photons in the lepton sample by requiring that a track must point to the electromagnetic cluster. Having good tracking detectors over similar coverage as electromagnetic calorimetry similarly aids in $y$ resolution at low $y$ from a lepton method only (as explained earlier), as the angular as well as the momentum resolution for trackers are much better than for electromagnetic calorimeters. The hadron suppression can be further improved by adding a Cherenkov detector to the electromagnetic calorimetry or having tracking detectors, (e.g., a Time Projection Chamber) to provide good $\mathrm{d} E / \mathrm{d} x$. Combining the responses from the electromagnetic calorimeter and the Cherenkov detectors or $\mathrm{d} E / \mathrm{d} x$ may especially help in the region of low-momentum scattered leptons, about $1 \mathrm{GeV} / c$. Other detector technologies, such as transition radiation detectors, may provide hadron rejection by a factor of 100 for leptons with $\gamma>1000$ $\left(\gamma=1 / \sqrt{\left(1-v^{2} / c^{2}\right)}\right)$.

There is specific interest in extracting structure functions with heavy quarks from semi-inclusive reactions for mesons, which contain charm or bottom quarks. To measure such structure functions as $F_{2}^{C}, F_{L}^{C}$, and $F_{2}^{B}$, it is sufficient to tag the charm and the bottom quark content via the detection of additional leptons (electrons, positrons, muons) in addition to the scattered (beam) lepton. The leptons from charmed mesons can be identified via a displaced vertex of the second lepton $(\langle\tau\rangle \sim 150 \mu \mathrm{m})$. This can be achieved by integrating a high-resolution vertex detector into the detector design. For measurements 

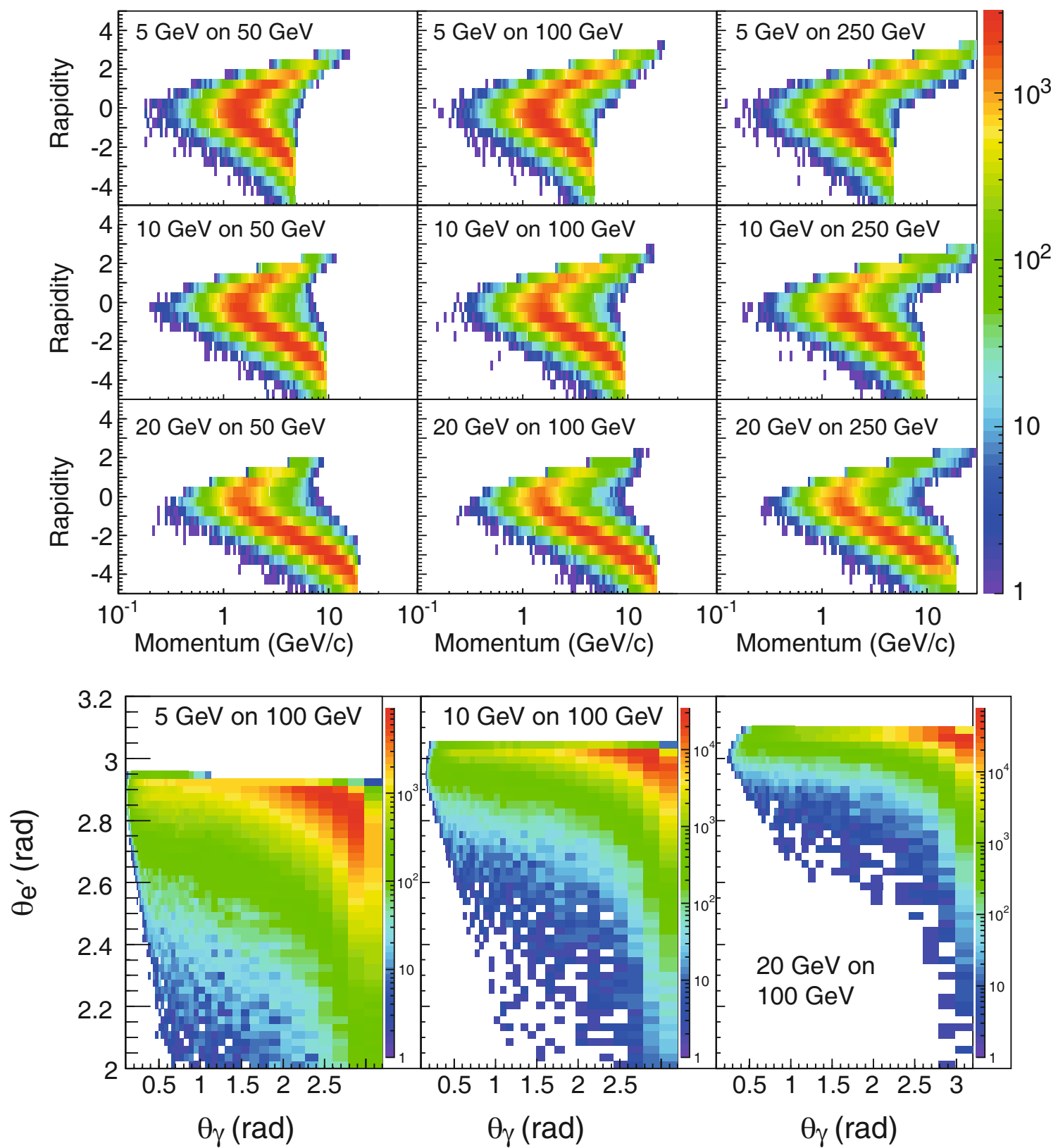

Fig. 92. The energy vs. rapidity in the laboratory frame for photons from DVCS for different center-of-mass energies (top) and the correlation between the scattering angle of the DVCS photon and the scattered lepton for three different center-of-mass energies. The following cuts have been applied: $Q^{2}>1.0 \mathrm{GeV}^{2}, 0.01<y<0.95, E_{\gamma}>1 \mathrm{GeV}$ and $-5<$ rapidity $<5$.

of the charmed (bottom) fragmentation functions, or to study medium modifications of heavy quarks in the nuclear environment, at least one of the charmed (bottom) mesons must be completely reconstructed to have access to the kinematics of the parton. This requires, in addition to measuring the displaced vertex, good particle identification to reconstruct the meson via its hadronic decay products, e.g. $D^{0} \rightarrow K^{ \pm}+\pi^{\mp}$.

Figure 92 shows the energy vs. rapidity distributions for photons from deeply virtual Compton scattering (DVCS), and the correlation between the scattering angle of the DVCS photon and the scattered lepton in the laboratory frame for different beam energy combinations.
The general patterns are as in fig. 90, but even at the low lepton beam energies the DVCS photons go more into the backward direction. However, for imaging studies through exclusive reactions involving light mesons, a $Q^{2}$ cut must be applied for a valid partonic interpretation. Since exclusive low- $Q^{2}$ hadrons are produced in the forward direction, a $Q^{2}>10 \mathrm{GeV}^{2}$ cut changes the kinematic patterns from fig. 90 .

The most challenging constraints on the detector design for exclusive reactions compared to semi-inclusive reactions is, however, not given by the final state particle $(\pi, K, \rho, \phi, J / \psi, \gamma)$, but to ensure the exclusivity of the event. 


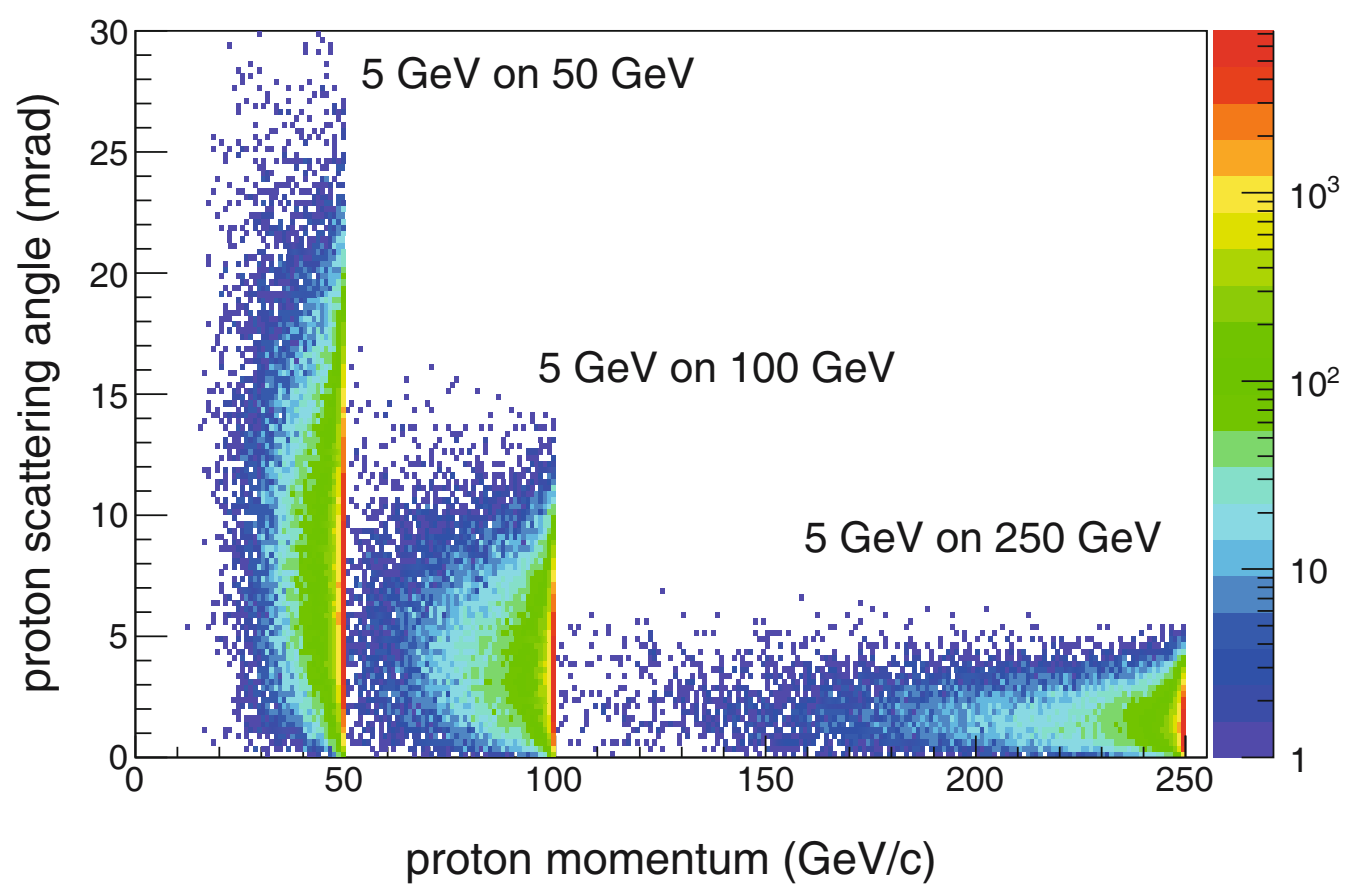

Fig. 93. The scattered proton momentum vs. scattering angle in the laboratory frames for DVCS events with different beam energy combinations. The following cuts have been applied: $1 \mathrm{GeV}^{2}<Q^{2}<100 \mathrm{GeV}^{2}, 0.00001<x<0.7$ and $0<t<2 \mathrm{GeV}^{2}$. The angle of the recoiling hadronic system is directly and inversely correlated with the proton energy. It thus decreases with increasing proton energy.

\subsection{Recoil baryon angles and $\mathrm{t}$ resolution}

For exclusive reactions on the nucleon or coherent nuclear processes, it is extremely important to ensure that the nucleon (or the nucleus) remains intact during the scattering process. Hence, one has to ensure exclusivity by measuring all products. In general, for exclusive reactions, one wishes to map the four-momentum transfer (or Mandelstam variable) $t$ of the hadronic system, and then obtain an image by a Fourier transform, for $t$ close to its kinematic limit $t_{\text {min }}$ up to about $1-2 \mathrm{GeV}$ (for details see chapt. 3.6 in ref. [2]).

Figure 93 shows one of the most challenging constraints on the detector and interaction region design from exclusive reactions, the need to detect the full hadronic final state. The figure shows the correlation between proton scattering angle and its momentum, and illustrates that the remaining baryonic states go in the very forward ion direction. Even at a proton energy of $50 \mathrm{GeV}$, the proton scattering angles only range to about $2^{\circ}$. At proton energies of $250 \mathrm{GeV}$, this number is reduced to one fifth. In all cases, the scattering angles are small. Because of this, the detection of these protons, or more general recoil baryons, is extremely dependent on the exact interaction region design and will therefore be discussed in more detail in the machine-dependent part of this section.

In the case of nucleus breakup as in, e.g., measurements of the quasi-free reaction on the nucleon in the nucleus, detection of the nuclear spectators and fragments is required. Unlike the recoil baryons from, e.g., DVCS the ion fragments have rigidities different from the beam.
Examples of these processes are spectator tagging with polarized ion beams requiring a resolution in the transverse momentum better than the Fermi momentum and detection of the final state in heavy-ion collisions.

\subsubsection{Luminosity measurement}

The Bethe-Heitler bremsstrahlung process $e p \longrightarrow e p \gamma$ was successfully used to measure luminosity by the experiments at the HERA $e+p$ collider. It has a large cross-section, allowing rapid measurements with negligible statistical uncertainty. The cross section of this process can be calculated entirely within QED, and is known to a precision of $\sim 0.2 \%$. The luminosity measurement was typically carried out by detecting the final-state photons; the final-state electron was also measured in some cases for experimental cross checks. Limitations in determining the geometric acceptance of the very forward photons resulted in a systematic uncertainty of $1-2 \%$ on the HERA luminosity measurements. For a polarized $e+p$ collider, the bremsstrahlung cross-section has a dependence on the beam polarizations, which may be expressed as $\sigma=\sigma_{0}\left(1+a P_{e} P_{p}\right)$. Preliminary estimates indicate that the coefficient $a$ is small, but detailed studies are currently underway to understand the size of $a$ relative to the magnitude of the double-spin asymmetries $A_{L L}$ at small $x_{B}$. The theoretical uncertainty on $a$, and the experimental uncertainties on the measured beam polarizations $P_{e}$ and $P_{p}$, will limit the precision of the absolute and relative luminosity measurements. 


\subsubsection{Hadron and lepton polarimetry}

Compton back-scattering is the established method to measure lepton beam polarization in $e+p$ colliders. At HERA, there were two Compton back-scattering polarimeters [340]: one measuring the transverse polarization (TPOL) of the beam through a position asymmetry and one measuring the longitudinal polarization (LPOL) of the beam through an energy asymmetry in Compton back-scattered photons. The TPOL and LPOL systematic uncertainties of RUN-I were $3.5 \%$ and $1.6 \%$ and of RunII $1.9 \%$ and $2.0 \%$, respectively. In spite of the expected high luminosity at the EIC, these systematic uncertainties could be reduced to $\sim 1 \%$ if special care is taken to reduce the impact of beam orbit instabilities and laser light polarization on the measurement. The detection of the lepton and the Compton photon in coincidence will provide an energy self-calibration of the polarimeter.

To measure the hadron beam polarization is very difficult as, contrary to the lepton case, there is no process that can be calculated from first principles. Therefore, a two-tier measurement is needed: one providing the absolute polarization, which has low statistical power and a high statistical power measurement, which measures the relative polarization. At RHIC [341], the single-spin asymmetry $A_{N}$ of the elastically scattered polarized proton beam on a polarized hydrogen jet is used to determine the absolute polarization. This measurement provides the average polarization per fill and beam with a statistical uncertainty on the order of $\sim 5 \%$ and a systematic uncertainty of $3.2 \%$. High-statistics bunch-by-bunch relative polarization measurements are provided, measuring the single-spin asymmetry $A_{N}$ for scattering the polarized proton beam of a carbon fiber target. To obtain absolute measurements, the $p \mathrm{C}$-measurements are cross normalized to the absolute polarization measurements from the hydrogen-jet polarimeter. The $p \mathrm{C}$-measurements provide the polarization lifetime and the polarization profile per fill with high statistical precision. The achieved total systematic uncertainty for single spin asymmetries is $3.4 \%$. The systematic uncertainties could be further reduced by monitoring continuously the molecular hydrogen contamination in the jet, improving the operational stability of the carbon fiber targets, and by developing methods to monitor the silicon detector energy calibration at the recoil carbon energy. All are under development for the polarized $p+p$ program at RHIC.

To have minimal impact from potential bunch-tobunch polarization fluctuations on the luminosity measurement, it is important to have both hadron and lepton beam polarimeters that can provide high-statistics polarization information for individual bunches.

\subsection{Detector and interaction region (IR) layout}

\subsection{1 eRHIC detectors and IR considerations and technologies}

Three studies on a possible implementation for an eRHIC detector have been performed. Two studies are built on the existing RHIC detectors. Both the
PHENIX and STAR Collaborations have studied how the sPHENIX [342] and STAR [343] detectors would have to be upgraded/modified to fulfill the performance requirements as laid out by the eRHIC physics program [344,345]. The third study is based on a green field design for an eRHIC detector, which is completely optimized to the physics requirements and the change in particle kinematics resulting from varying the center-of-mass energies from $55 \mathrm{GeV}$ to $140 \mathrm{GeV}$. In the following mainly details about the model detector will be described.

Combining all the requirements described in sect. 6.2 and in the preceding physics sections, a schematic view of the emerging dedicated eRHIC detector is shown in fig. 94.

The compact tracker, located symmetrically with respect to the IP, consists of: a MAPS silicon barrel vertex detector and a set of forward/backward disks; a $2 \mathrm{~m}$ long TPC with a gas volume outer radius of $0.8 \mathrm{~m}$ and several GEM stations, all placed into a $\sim 3 \mathrm{~T}$ solenoid field. The TPC is specifically chosen as the main tracking element because of its small overall material budget, minimizing the rate of photon conversions on detector components, which is required in particular for the DVCS measurements. Besides this, the TPC should provide good charged PID in the momentum range up to a few $\mathrm{GeV} / c$ at central rapidities. Other detector options for the main tracker, such as a set of cylindrical micromegas planes are considered as well [347]. Significant progress in the last decade in the development of Monolithic Active Pixel Sensors (MAPS) in which the active detector, analog signal shaping, and digital conversion take place in a single silicon chip (i.e., on a single substrate; see [348] and references therein) provides for a unique opportunity for a $\mu$-vertex detector for an eRHIC detector. As a result, CMOS pixel detectors can be built with high segmentation, limited primarily by the space required for additional shaping and digital conversion elements. The key advantage of CMOS MAPS detectors is the reduced material required for the detector and the (on substrate) on-detector electronics. Such detectors have been fabricated and extensively tested (see, e.g., [349]) with thicknesses of about $50 \mu \mathrm{m}$, corresponding to $0.05 \%$ of a radiation length. The vertex detector, covering the central rapidity range $-1<\eta<1$, is strongly inspired by the STAR HFT tracker design [350] a similar design is now considered by the ALICE experiment at LHC. The projected rates for a luminosity in the $10^{34} \mathrm{~cm}^{-2} \mathrm{~s}^{-1}$ range, depending on the center-of-mass energy, between 300 and $600 \mathrm{kHz}$, with an average of 6 to 8 charged tracks per event. These numbers do not impose strong constraints on any of the above technology for tracking detectors.

To have equal rapidity coverage for tracking and electromagnetic calorimetry will provide good electron identification and give better momentum and angular resolutions at low inelasticity, $y$, than with an electromagnetic calorimeter alone. Therefore the detector will be equipped with a set of electromagnetic calorimeters, hermetically covering a pseudorapidity range of at least $-4<\eta<4$. The calorimeter technology choice is driven by the fact that a moderately high-energy resolution, on the order of $\sim 2-3 \% / \sqrt{E}$, is needed only at backward (electron-going) 


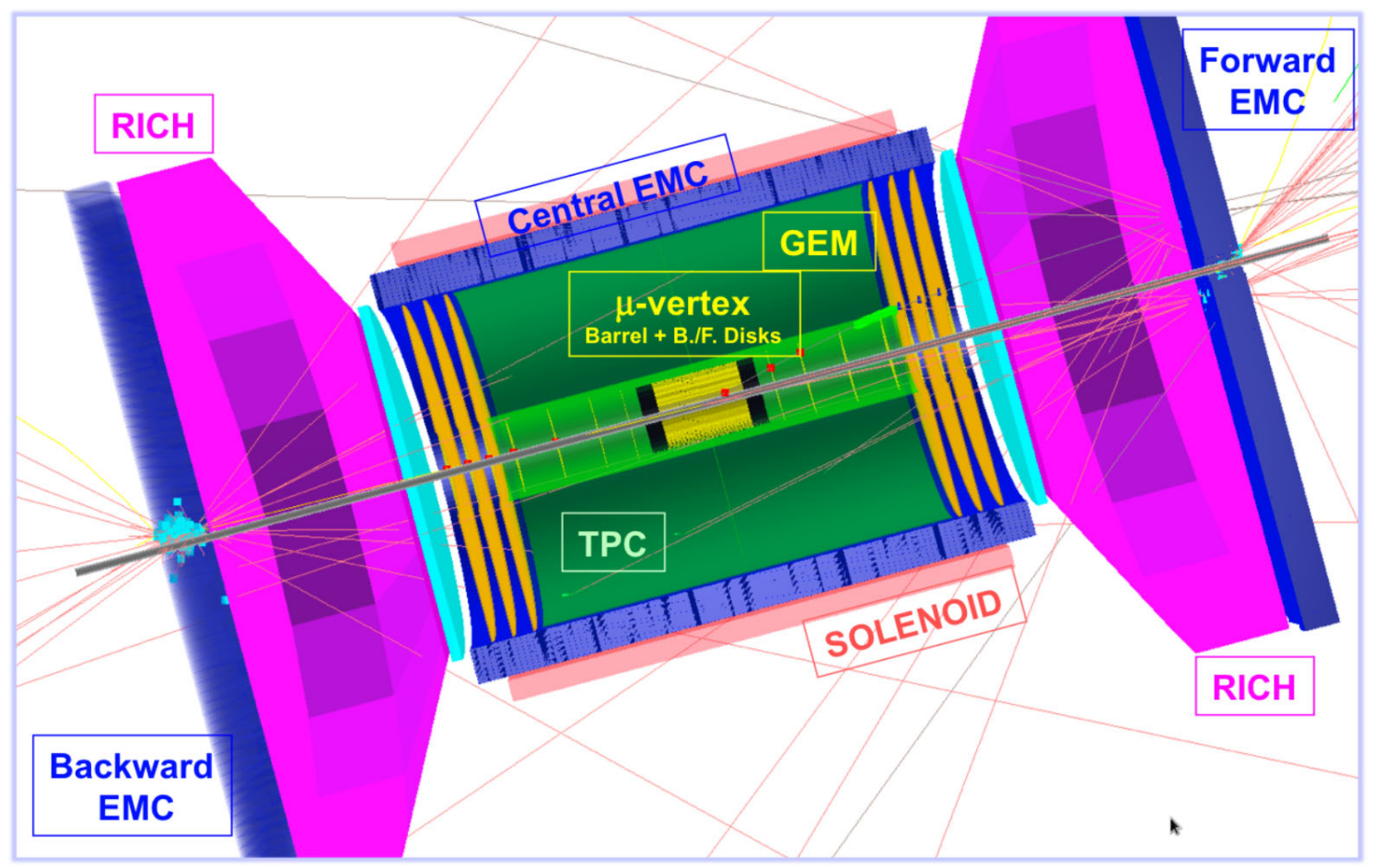

Fig. 94. The eRHIC model detector implementation (BeAST = Brookhaven eA Solenoidal Tracker) with tracker and calorimeter components implemented in the EicRoot GEANT simulation framework [346].

rapidities. Therefore in the present design the backward endcap calorimeter for the $-4<\eta<-1$ range is composed of PWO crystals at room temperature, with the basic performance parameters taken from the very extensive PANDA R\&D studies [351]. The calorimeter is located $\sim 500 \mathrm{~mm}$ away from the IP. The crystal length corresponds to $\sim 22.5 \chi_{0}$, and both the crystal shape and grouping follow the ideas of the PANDA and CMS [352] calorimeter designs. For the barrel and forward endcap electromagnetic calorimeters, covering a pseudo-rapidity range of $-1<\eta<4$, a noticeably worse energy resolution suffices. In order to save costs, at present it is planned to use the STAR upgrade R\&D building blocks of tungsten powder scintillating fiber sampling calorimeter towers, with a design goal of $\sim 12 \% / \sqrt{E}$ energy resolution. The forward endcap calorimeter will be located at $\sim 2500 \mathrm{~mm}$ from the IP in hadron-going direction. The barrel calorimeter will have an average installation radius of $\sim 900 \mathrm{~mm}$ and be composed of slightly tapered towers, in order to avoid gaps in the azimuthal direction. Both calorimeter types will have a non-projective geometry and tower length corresponding to $\sim 23 \chi_{0}$.

To quantify the performance of the model detector to reconstruct the event kinematics PYTHIA events, generated for a $15 \mathrm{GeV}$ electron beam colliding with a $250 \mathrm{GeV}$ proton beam, were passed through the detector simulation. Figure 95 shows as example the results of detector smearing on event kinematics calculated using the electron method (left) and the Jacquet-Blondel method (right), crucial for charge current (CC) events. As expected, due to the excellent resolution in both momentum and electron energy, $y, x$ and $Q^{2}$ are exceedingly well reconstructed.
Event purity is excellent at moderate-to-large $y$ (typically $>90 \%$ ) even with a relatively fine $x-Q^{2}$ binning of five bins per decade in $x$ and four per decade in $Q^{2}$. The Jacquet-Blondel (JB) method is a purely hadronic method of kinematic calculation, meaning it can be used in the absence of a measured scattered lepton. A drawback of this method is that it suffers from very poor resolution at low $Q^{2}$. Fortunately, as the majority of the CC cross-section resides at large $Q^{2}>100$, the JB method can be very successfully applied to the analysis of these events [78].

To increase the separation of photons and $\pi^{0}$ 's to high momenta and to improve the matching of charged tracks to the electromagnetic cluster, it would be an advantage to add, in front of all calorimetry, a high-resolution preshower. To have at least one pre-shower layer with 1-2 radiation lengths of tungsten and silicon strip layers (possibly with two spatial projections) would allow to separate single photons from $\pi^{0}$ to up $p_{T} \approx 50 \mathrm{GeV}$, as well as enhanced electron-identification. A straw-man design could have silicon strips with $\Delta \eta=0.0005$ and $\Delta \phi=0.1$.

Due to the momentum range to be covered the only solution for PID in the forward direction is a dual radiator $\mathrm{RICH}$, combining either aerogel with a gas radiator like $\mathrm{C}_{4} \mathrm{~F}_{10}$ or $\mathrm{C}_{4} \mathrm{~F}_{8} \mathrm{O}$ if $\mathrm{C}_{4} \mathrm{~F}_{10}$ is no longer available, or combining the gas radiator with a liquid radiator like $\mathrm{C}_{6} \mathrm{~F}_{14}$. In the barrel part of the detector, several solutions are possible as the momenta of the majority of the hadrons to be identified are between $0.5 \mathrm{GeV}$ and $5 \mathrm{GeV}$. The technologies available in this momentum range are high-resolution ToF detectors $(t \sim 10 \mathrm{ps})$, a DIRC or a proximity focusing Aerogel RICH. 

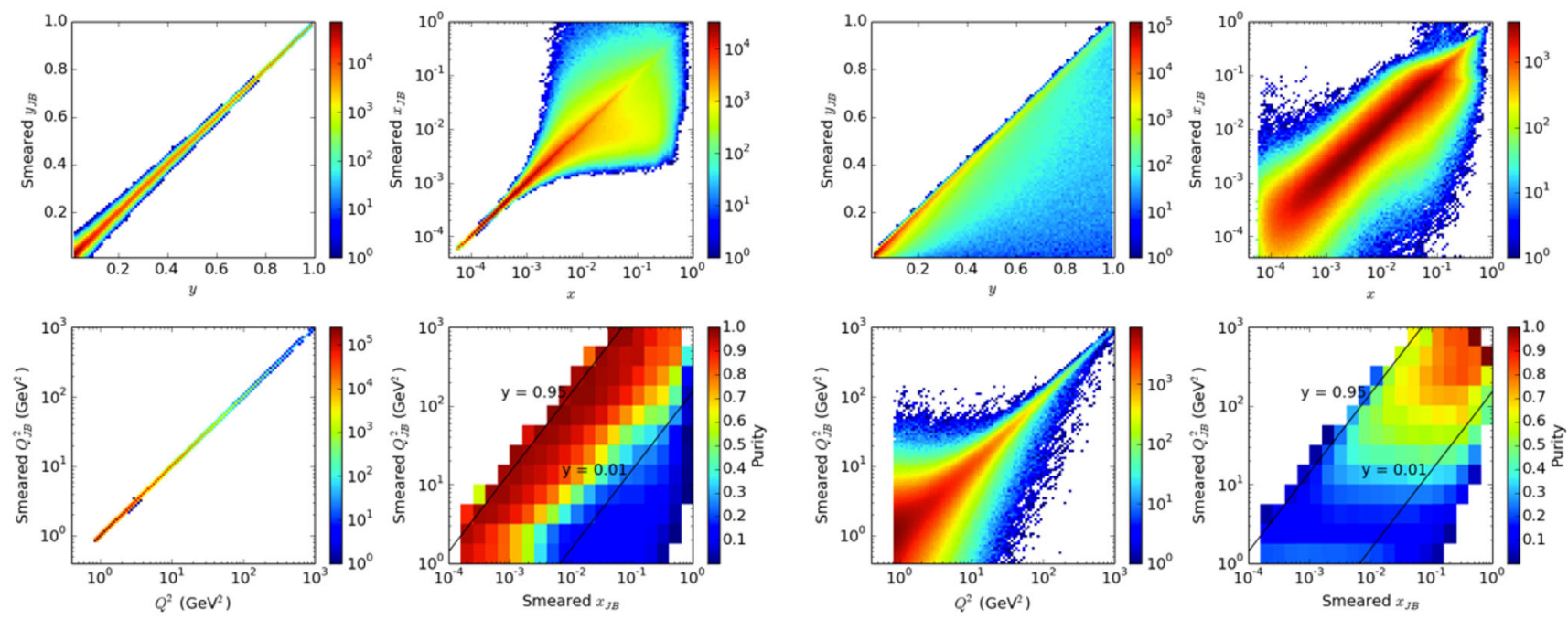

Fig. 95. The correlation between smeared and true $y, x$ and $Q^{2}$ (top to bottom left), and the resulting bin-by-bin event purity in the $x-Q^{2}$ plane (bottom right), reconstructed using the electron method. Purity is defined as $\left(N_{\text {gen }}-N_{\text {out }}\right) /\left(N_{\text {gen }}-N_{\text {out }}+N_{\text {in }}\right)$, where $N_{\text {gen }}$, out, in are the number of events generated in a bin, smeared out of it, and smeared into it from other bins, respectively. Both the electron (left) and Jacquet-Blondel (right) method are shown.

To achieve the physics program as described in earlier sections, it is extremely important to integrate the detector design into the interaction region design of the collider. Particularly challenging is the detection of forward-going scattered protons from exclusive reactions, as well as of decay neutrons from the breakup of heavy ions in non-diffractive reactions. The eRHIC design features a $10 \mathrm{mrad}$ crossing angle between the protons or heavy ions during collisions with electrons. This choice removes potential problems for the detector induced by synchrotron radiation. To obtain luminosities higher than $10^{34} \mathrm{~cm}^{-2} \mathrm{~s}^{-1}$, very strong focusing close to the IR is required to have the smallest beam sizes at the interaction point. A small beam size is only possible if the beam emittance is also very small. The focusing triplets are symmetrically around the interaction point (IP) starting at 4.5 meters.

While the above accomplishes a small-emittance electron beam, the ions and protons need to be cooled by coherent electron cooling to have small emittance. The eRHIC interaction region design relies on the existence of small emittance beams with a longitudinal RMS of $\sim 5 \mathrm{~cm}$, resulting in a $\beta^{*}=5 \mathrm{~cm}$. Strong focusing is obtained by three high-gradient quadrupole magnets using recent results from the LHC quadrupole magnet upgrade program (reaching gradients of $200 \mathrm{~T} / \mathrm{m}$ at $120 \mathrm{~mm}$ aperture). To ensure the previously described requirements from physics are met, four major requirements need to be fulfilled: high luminosity (> 100 times that of HERA); the ability to detect neutrons; measurement of the scattered proton from exclusive reactions (i.e., DVCS); and the detection of spectator protons from deuterium and He3 breakup. The eRHIC IR design fulfills all these requirements. The apertures of the interaction region magnets allow detection of neutrons with a solid angle of $\pm 4 \mathrm{mrad}$, as well as the scattered proton from exclusive reactions, i.e.
DVCS, up to a solid angle of $\sim 9$ mrad. The detection of the scattered proton from exclusive reactions is realized by integrating several "Roman Pot" stations into the warm section of the IR. The electrons are transported to the interaction point through the heavy-ion/proton triplets, seeing zero magnetic field.

Figure 79 shows the current eRHIC interaction region design in the direction of the outgoing hadron beam. The other side of the IR is mirror symmetric for the incoming hadron beam. A low scattering-angle lepton tagger for events with $Q^{2}<0.1 \mathrm{GeV}^{2}$ is currently integrated in the outgoing lepton beam line design. An extensive R\&D program has been started to design and integrate the lepton beam polarimeter and the luminosity monitor into the interaction region [353].

\section{ePHENIX}

The PHENIX Collaboration has proposed to build an eRHIC detector, here referred to as ePHENIX, upon sPHENIX [342], which is designed to further advance the study of cold and hot nuclear matter in nuclear collisions, with its main emphasis on jet measurements. In addition to fully utilizing the sPHENIX superconducting solenoid and barrel calorimetry, ePHENIX adds new detectors in the barrel, electron-going and hadron-going directions [344], see fig. 96. In the electron-going direction, a crystal calorimeter is added for electron identification and precision resolution. A compact time projection chamber, augmented by additional forward and backward angle GEM detectors, provides full tracking coverage. In the hadron-going direction, behind the tracking is electromagnetic and hadronic calorimetry. Critical particle identification capabilities are incorporated via a barrel DIRC, and in the hadron-going direction, a gas RICH and an aerogel RICH. 


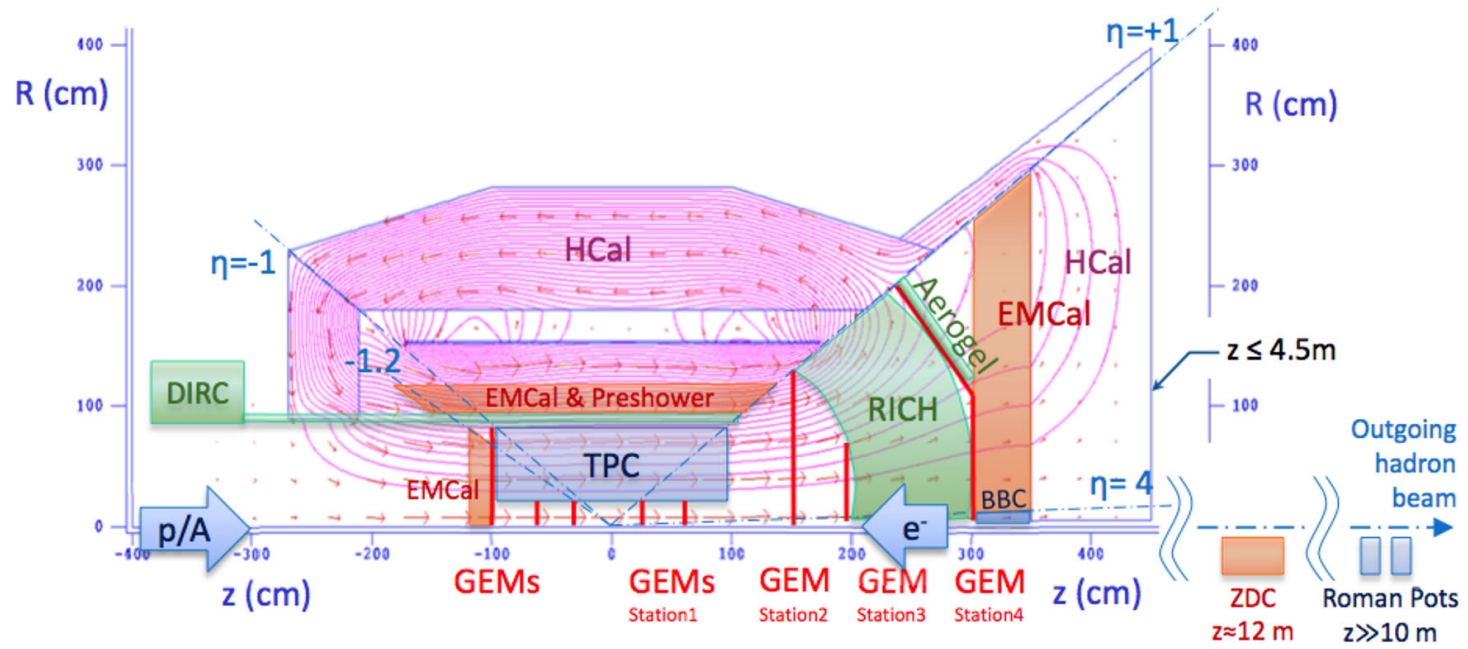

Fig. 96. A cross-section through the top-half of the ePHENIX detector concept, showing the location of the superconducting solenoid, the barrel calorimeter system, the EMCal in the electron-going direction and the system of tracking, particle identification detectors and calorimeters in the hadron-going direction. Forward detectors are also shown along the outgoing hadron beamline. The magenta curves are contour lines of magnetic field potential as determined using the 2D magnetic field solver, POISSON

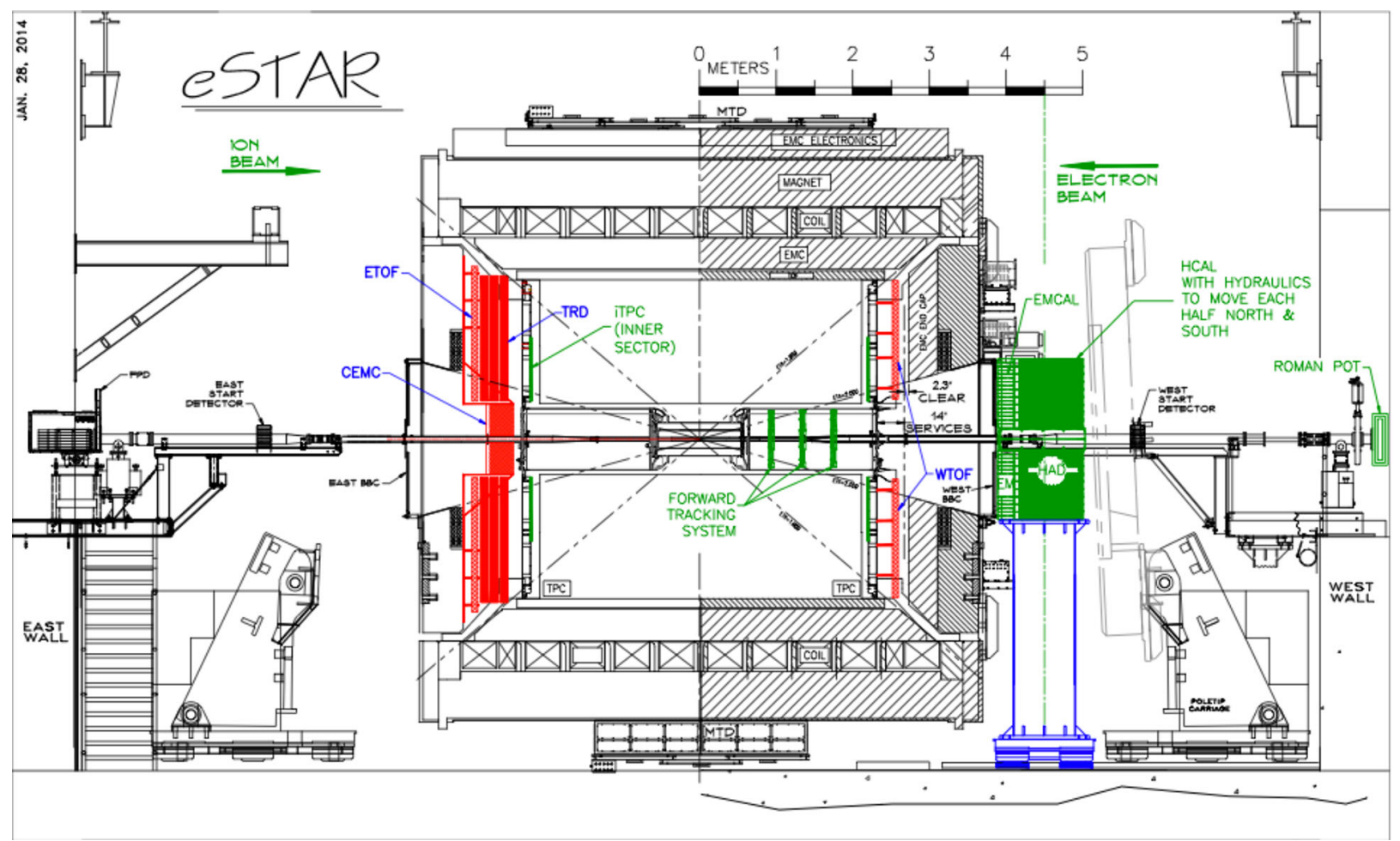

Fig. 97. The eSTAR layout with the proposed upgrades of iTPC, Forward Calorimetry System (FCS), the Forward Tracking System (FTS), Endcap TOF (E/W TOF), BSO Crystal Calorimeter (CEMC), GEM-based TRD. In this configuration, the electron beam is from right to left (eastward) while hadron beam from left to right (westward).

eSTAR

The STAR Collaboration has proposed a path to evolve the existing STAR detector [342] to an initialstage eRHIC detector, eSTAR. In this plan an optimized suite of detector upgrades will maintain and extend the existing low-mass mid-central rapidity tracking and particle-identification capabilities towards more forward rapidities in both the electron and hadron going beam directions. This plan is described in [344], which contains also a capability assessment for key measurements of the eRHIC science program. Figure 97 shows a side-view of the baseline eSTAR detector layout. This baseline plan consists of three essential upgrade projects, namely end- 


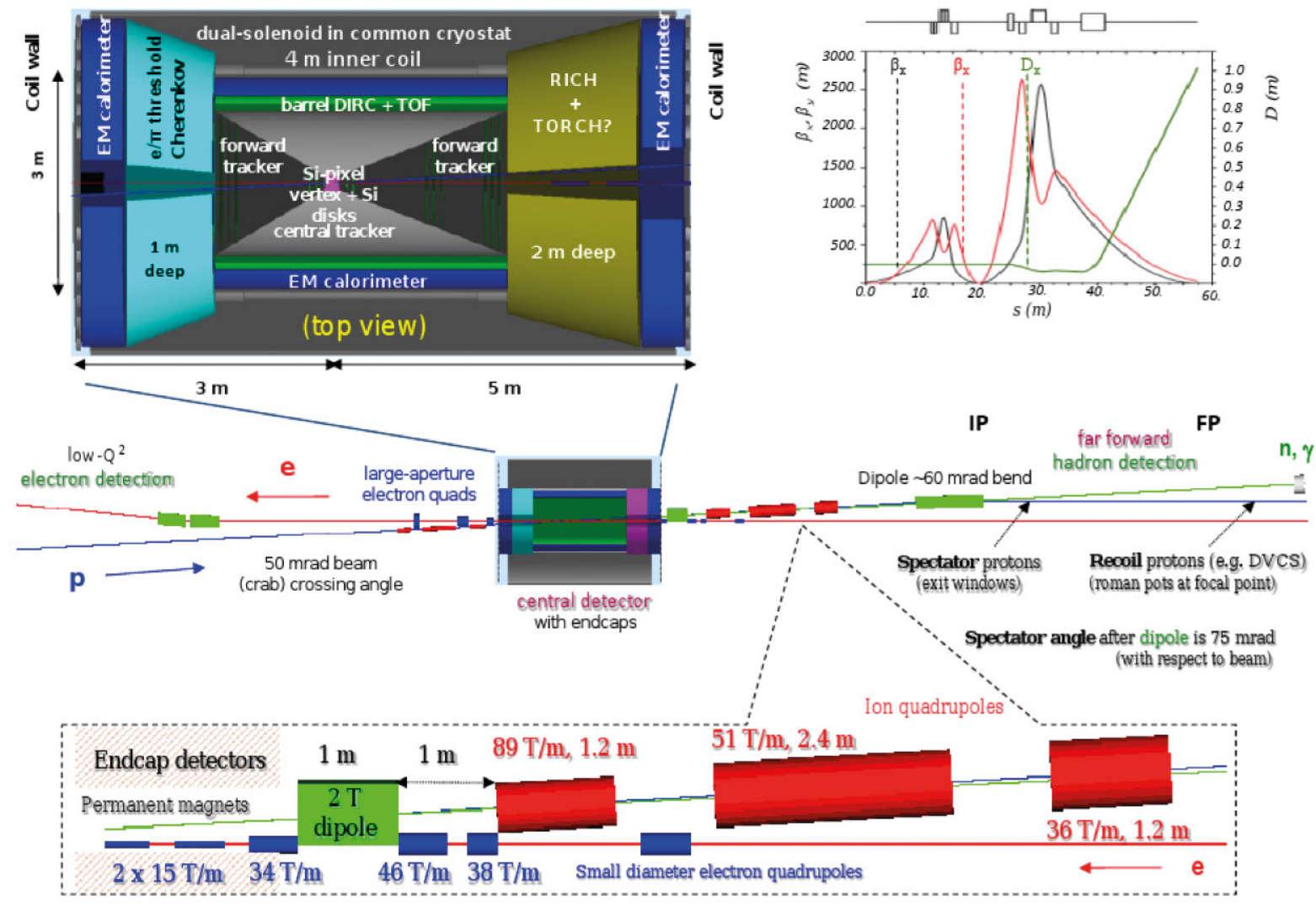

Fig. 98. The interaction region and central detector layout, and its placement in the general integrated detector and interaction region. The central detector includes endcaps in both the electron and ion direction.

cap Time-of-Flight walls located between the TPC and the magnet pole-tips on the East and West sides of the interaction region (ETOF and WTOF, covering the regions $1<|\eta|<2$ in pseudo-rapidity), a GEM-based Transition Radiation Detector (GTRD) between the TPC and ETOF in the forward electron direction, covering $-2<\eta<-1$, and a Crystal ElectroMagnetic Calorimeter with preshower (CEMC, covering $-4<\eta<-2$ ). Furthermore, eSTAR will rely on a replacement upgrade of the Inner Sectors of the existing Time-Projection-Chamber prior to a completion of the RHIC Beam-Energy Scan program with $\mathrm{A}+\mathrm{A}$ collisions and on a subsequent upgrade in the form of a new Forward Calorimeter System (FCS) with associated Forward Tracking System (FTS) on the West side of STAR.

\subsubsection{Detector design for JLEIC}

A global outline of the fully integrated JLEIC detector and interaction region (IR) is given in fig. 98. A detailed description of the central detector as well as the extended interaction region strategy for achieving a fullacceptance detector can be found in ref. [2]. Since the publication of this article the central detector design has been optimized by, e.g., using innovative design features to relax specifications and/or to improve its performance.
All basic requirements and technologies/solutions are understood. Furthermore, new opportunities for small-angle hadron and electron detection have been identified, and inter-lab and university collaborations on general detector R\&D have been formed. The subsequent sections will focus on the main aspects of one detector compatible with the full-acceptance interaction region and optimized for the physics goals of SIDIS and exclusive reactions (see sect. 2) while keeping in mind that accelerator integration is the highest priority since it allows the storage ring to be designed around the detector needs. Since a ringring collider configuration can support multiple detectors without time sharing, the full-acceptance detector could be complemented by, for instance, a high-luminosity detector at another interaction point. Such a second detector could use Time Projection Chambers and focus on hadron calorimetry (jets).

To achieve full-acceptance, small-angle detection is required on either side of the central detector. The low- $Q^{2}$ electron detection required for heavy-flavor photoproduction processes is relatively simple to incorporate, including a dipole chicane for tagger electrons, which would also be used for a Compton polarimeter. The latter would have a laser in the middle of the chicane, where the polarization would be identical to that at the IP. In addition to the photons, the Compton electrons would also be detected. The space on the side of the low- $Q^{2}$ tag- 
ger will also be instrumented for luminosity monitoring. Measuring forward and ultra-forward going hadronic or nuclear fragments along the ion direction is more challenging and we make critical use of various ingredients of the JLEIC detector/interaction region design: i) the $50 \mathrm{mrad}$ crossing angle, which moves the spot of poor resolution along the solenoid axis into the periphery and minimizes the shadow from the electron magnets (see, e.g., sect. 5.2.5); ii) the range of proton energies (see, e.g., sect. 5.2.2); iii) a small $2 \mathrm{Tm}$ dipole magnet before the ion final focusing quadrupole magnets (FFQs) to allow high-resolution tracking of particles that do not enter the FFQs; iv) low-gradient FFQs with apertures sufficient for particles scattered at initial angles of $10-15 \mathrm{mrad}$ in each direction for all ion fragment rigidities; and v) a $20 \mathrm{Tm}$ large-acceptance dipole magnet a few meters downstream of the FFQs to peel off spectator particles and allow for very small-angle detection with high resolution (essentially only limited by the intrinsic momentum spread of the beam).

As illustrated in fig. 98 detectors will be placed in front of the FFQs, between the FFQs and the $20 \mathrm{Tm}$ dipole, and/or in an extended, magnet-free drift space downstream of the latter providing far forward hadron detection. In particular, the FFQ acceptance for neutral particles will depend on the choice of the peak field ( $6 \mathrm{~T}$ baseline) but is generally in the $\pm 10-15$ mrad range, centered close to zero. The neutrons (and boosted nuclear photons) will be detected in a zero-degree calorimeter (ZDC) on the outside of the ring. In this configuration, any desired angular resolution can be achieved simply by adjusting the distance of the ZDC (as well as its size). This then results in an essentially $100 \%$ full-acceptance detector.

To minimize synchrotron radiation and improve the small-angle hadron acceptance and resolution, the electron beam travels along the center of the central solenoid, while the proton/ion beam traverses it at the crab crossing angle.

To fulfill the requirement of hermeticity, the central detector will be built around a solenoid magnet (with a coil length of about $4 \mathrm{~m}$ ). Due to the asymmetric beam energies, the interaction point (IP) will be slightly offset towards the electron side $(1.5 \mathrm{~m}+2.5 \mathrm{~m})$. This will allow more distance for the tracking of high-momentum hadrons produced at small angles, and a larger bore angle for efficient detection of the scattered beam leptons. Existing superconducting detector solenoid magnets like those from CLEO or BaBar ${ }^{19}$ would be suitable for use in the JLEIC at either IP. Like many detector solenoids these employ an iron yoke for the flux return, which encapsulates the detector and the endcaps. An interesting alternative is a dual solenoid where the inner and outer solenoid have opposite polarity thus providing an iron-free flux return in the space between them. This design was proposed for the 4 th detector concept for the ILC. The main advantages of the dual solenoid include light weight, high field capability $(3 \mathrm{~T})$, improved endcap acceptance, compact endcaps

\footnotetext{
19 Both are $4 \mathrm{~m}$ long, have a $3 \mathrm{~m}$ diameter, a $1.5 \mathrm{~T}$ field, and an iron yoke.
}

(coils instead of iron), easy detector access, low external field, and precise internal field map (no hysteresis). These features are ideal for a detector optimized for SIDIS, e.g., partonic fragmentation, and exclusive processes with recoils (see sect. 6.3). The initial magnetic design for the JLEIC dual-solenoid- based detector has been completed.

Figure 98 shows the dual-solenoid-based JLEIC detector with three layers of forward- and central trackers including a vertex detector. The current tracker layout is compatible with both a dual solenoid and the CLEO magnet. Particle identification in the central detector would be provided by a TOF, and a radially compact detector providing $e / \pi, \pi / K$, and $K / p$ identification. The current baseline design includes a DIRC whose performance at an EIC compared with state of the art (BaBar) is the topic of an R\&D proposal. Optimizations and alternatives to the global baseline design are discussed in more detail in ref. [2].

Small-angle tracking in the central detector could be an extension of the vertex tracker, using semiconductor detectors, while larger angles could be covered by planar micro-pattern detectors (GEMs/micromegas). On the electron side, where the particle momenta are generally lower, one could even consider drift chambers with a small cell size, in particular for a final tracking region that could be added outside of the solenoid itself. Lepton identification in the end-cap will be performed using an electromagnetic calorimeter and a High-Threshold Cherenkov Counter (HTCC) with $\mathrm{CF}_{4}$ gas or equivalent. The details of hadron identification in the electron end-cap can be found in ref. [2].

The ion-side end-cap would have to deal with hadrons with a wide range of momenta, some approaching that of the ion beam. While the small-angle tracking resolution on this side is greatly enhanced by the $50 \mathrm{mrad}$ crossing angle ${ }^{20}$ and dipole in front of the FFQs, the forward tracking would nevertheless greatly benefit from good position resolution, making this a priority. To identify particles of various species over the full momentum range, one would ideally want to use a RICH with several radiators, such as aerogel, $\mathrm{C}_{4} \mathrm{~F}_{10}$, and $\mathrm{CF}_{4}$. Possible implementations are detailed in ref. [2].

On the ion side, the detection will be performed in three stages. The first stage is the end-cap, which will cover all angles down to the acceptance of the forward spectrometer (several degrees around the ion beam line). This in turn has two stages: one upstream of the ion FFQs, and one downstream of them. The acceptance of all stages is matched so that there are minimal gaps in the coverage. The last stage will cover angles up to 10-15 mrad on either side of the beam (more vertically) for all ion fragments with different charge-to-mass ratios and fractions of the beam momentum, with modest requirements on magnet peak fields ${ }^{21}$

\footnotetext{
20 Particles scattered at zero degrees are not moving parallel to the $B$-field.

${ }^{21}$ Good performance can be achieved with peak fields for two magnets at $6 \mathrm{~T}$ and one at $5 \mathrm{~T}$.
} 

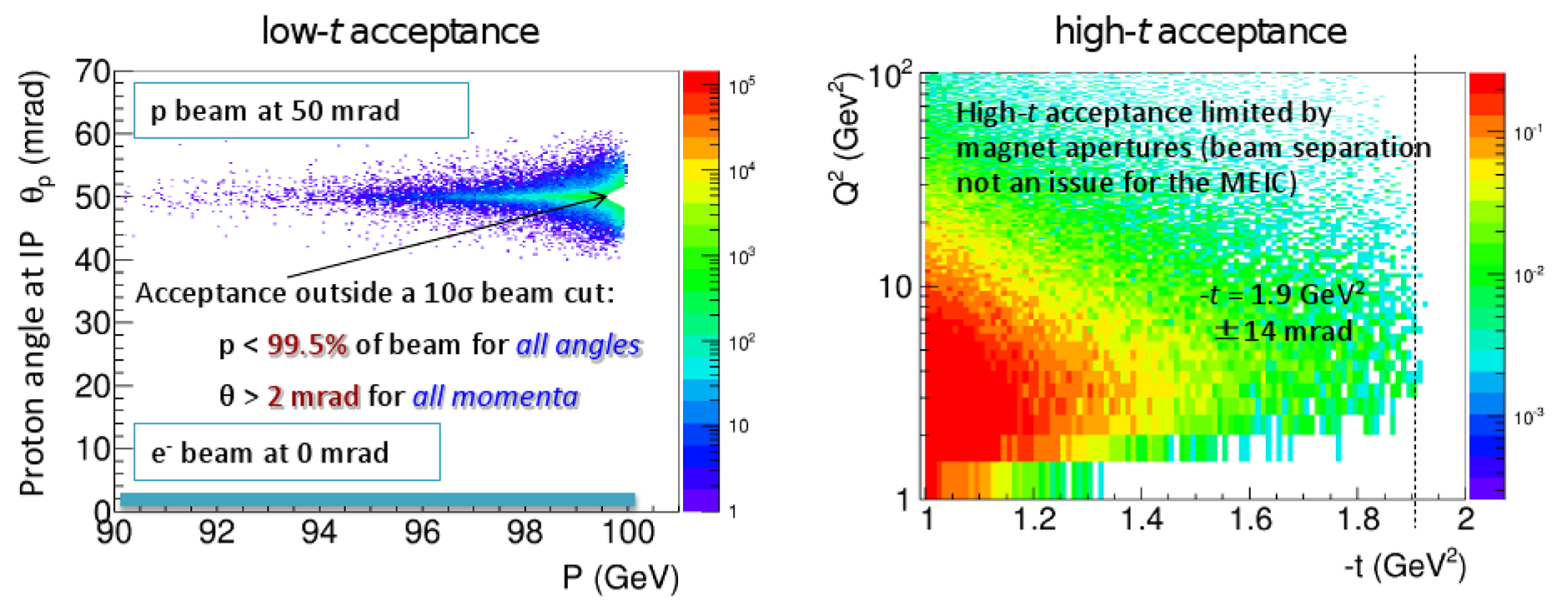

Fig. 99. The projected recoil baryon acceptance outside a $10 \sigma$ beam cut for the JLEIC for $5 \mathrm{GeV}$ electrons colliding with $100 \mathrm{GeV}$ protons at a crossing angle of $50 \mathrm{mrad}$. The following cuts have been applied: $Q^{2}>1 \mathrm{GeV}^{2}, x<0.1$, and $E_{e}^{\prime}>1 \mathrm{GeV}$.

The intermediate stage will use a $2 \mathrm{Tm}$ dipole to augment the solenoid at small angles where the tracking resolution otherwise would be poor. The magnet will be about $1 \mathrm{~m}$ long and its aperture will cover the distance to the electron beam (corresponding to the horizontal crossing angle of $50 \mathrm{mrad}$ ), while the acceptance in the other three directions is not restricted and can be larger. An important feature of the magnet design is to ensure that the electron beam line stays field free. The dipole will have trackers at the entrance and exit, followed by a calorimeter covering the ring-shaped area in front of the first ion FFQ. The intermediate stage is essential for providing a wide coverage in $-t$ also for the lowest beam energies, and to investigate target fragmentation.

The last, small-angle stage provides the ultra-forward detection that is crucial for detecting recoil baryons and tagging of spectator protons in deuterium, as well as other nuclear fragments. The design is heavily integrated with the accelerator, and the $4 \mathrm{~m}$ long, $20 \mathrm{Tm}$ downstream dipole serves not only as a spectrometer, but also "corrects" the $50 \mathrm{mrad}$ crossing angle, and allows the neutrons to escape on a tangent to the ring, separating cleanly from the beam area before detection. This makes the electron and ion beam lines parallel in the $\sim 15 \mathrm{~m}$ long drift space after the dipole, with separation of more than $1 \mathrm{~m}$, providing ample space for detectors. To optimize the low- $t$ coverage, it is essential that the $10 \sigma$ (see footnote ${ }^{22}$ ) beam size is as small as possible. This is achieved through cooling (which also reduces the angular spread), and by introducing a weaker secondary focus $16 \mathrm{~m}$ downstream of exit from the large $20 \mathrm{Tm}$ analyzing dipole. Even the preliminary optics give full angular acceptance for charged particles with rigidities (momenta) of up to $99.5 \%$ of the beam momentum (or more than 100.5\%) down to zero degrees, and full momentum acceptance for particles scattered at more than about $2-3 \mathrm{mrad}$ with respect to the central beam. As shown in fig. 99, the high $-t$ recoil baryon acceptance is only limited by the magnet apertures, while the

\footnotetext{
22 The proton beam size at $60 \mathrm{GeV}$ (the mid-range point for
} the $20-100 \mathrm{GeV}$ JLEIC coverage). low $-t$ acceptance requiring small beam size at the detection point and large dispersion after the IP to move the recoils away from the beam is limited by the beam itself. The dipole aperture can also be made sufficiently large to accept all off-angle and off-momentum particles that exit the FFQs with the exception of some "spectator" protons from deuterium scattered at very large angles. These can, however, easily be detected in between the FFQs and the dipole. Tracking studies show that the momentum resolution for particles up to the beam momentum will only be limited by the intrinsic momentum spread of the beam (longitudinal $4 \times 10^{-4}$ ), and the angular resolution will also be excellent $(0.2 \mathrm{mrad}$ for all $\phi)$. This is very important since the four-momentum transfer of the hadronic system is proportional to $t \sim \theta_{p}^{2} E_{p}^{2}$, and the $t$-resolution for instance determines the quality of the $3 \mathrm{D}$ imaging that can be achieved (see sect. 6.3).

This white paper is a result of a community wide effort through the many EIC workshops organized by the physics communities associated with both BNL and JLab, which culminated in the INT Report [2]. We thank the following colleagues who made valuable comments to the draft of this document: Christine Aidala (Univ. of Michigan), Yasuyuki Akiba (RIKEN, Japan) Kieran Boyle (RIKEN BNL Research Center at BNL), JianPing Chen (Jefferson Laboratory) Leonard Gamberg (Penn State University) Yuji Goto (RIKEN, Japan), John Harris (Yale University), Thomas Hemmick (Stony Brook University), Barbara Jacak (Stony Brook University), Peter Jacobs (Lawrence Berkeley Laboratory), Zhongbo Kang (Los Alamos National Laboratory), David Kaplan (Institute of Nuclear Theory \& U. of Washington) Dmitri Kharzeev (BNL \& Stony Brook University), Sebastian Kuhn (Old Dominion University), Paul Newman (Univ. of Liverpool, United Kingdom), Joakim Nystrand (Univ. of Bergen, Norway), Kent Paschke (Univ. of Virginia), Krishna Rajagopal (Massachusetts Institute of Technology), Seamus Riordan (Stony Brook University) Oscar Rondon (Univ. of Virginia), Patrizia Rossi (Jefferson Laboratory), Bernd Surrow (Temple University) Michael Tannenbaum (BNL), Mikhail Tokarev (JINR, Russia), Xin-Nian Wang (Lawrence Berkeley National Lab \& Central China Normal Univ.). 
This work was supported in part by the U.S. Department of Energy, contract numbers DE-AC02-06CH11357 (RH), DE-AC02-98CH10886 (ECA, TB, SF, YH, MACL, JHL, YL, VL, TWL, BM, VP, J-WQ, ThR, MS, TT, DT, ThU, RV, SV, LZ), DE-AC02-05CH11231 (ES, FY), DEAC02-76SF00515 (MSu), DE-AC05-06OR23177 (AA, RE, VG, AH, F-LL, RM, VSM, PN-T, AP, CW, Y-HZ), and grant numbers DE-FG02-05ER41372 (ADe), DE-FG02-09ER41620 (ADu), DE-FG02-03ER41231 (HG), DE-SC0004286 (YuK), DE-FG02-88ER40415 (KK), DE-FG02-94ER40844 (Z-EM), DE-FG02-92ER40699 (AHM), DE-FG02-08ER41531 (MR-M), by the National Science Foundation, grant number PHY1019521 (TH), by The ERC grant HotLHC ERC-2001StG-279579; Ministerio de Ciencia e Innovacion of Spain grants FPA2008-01177, FPA2009-06867-E and ConsoliderIngenio 2010 CPAN CSD2007-00042; Xunta de Galicia grant PGIDIT10PXIB206017PR; and FEDER (NA), by Chilean CONICYT: grant number FB0821, Anillo grant ACT-119 and Fondecyt grant 1120953 (WB), by City University of NY PSCCUNY Research Award Program Grant 65041-0043 (ADu), by Chilean Fondecyt Grant 11121448 (HH), by Fondecyt (Chile) Grant No. 1090291 (BK), by Croatian Ministry of Science, contract no. 119-0982930-1016 (KKu), and by CEA-Saclay and GDR 3034 PH-QCD (FS).

Open Access This is an open access article distributed under the terms of the Creative Commons Attribution License (http://creativecommons.org/licenses/by/4.0), which permits unrestricted use, distribution, and reproduction in any medium, provided the original work is properly cited.

\section{References}

1. NSAC Long Range Plan (2007) http://science. energy.gov/np/nsac/.

2. D. Boer et al., arXiv:1108.1713 (2011) A report on the joint BNL/INT/Jlab program on the science case for an Electron-Ion Collider.

3. M. Glück, E. Reya, A. Vogt, Eur. Phys. J. C 5, 461 (1998) arXiv:hep-ph/9806404.

4. P. Jimenez-Delgado, E. Reya, Phys. Rev. D 79, 074023 (2009) arXiv:0810.4274.

5. European Muon Collaboration (J. Ashman et al.), Phys. Lett. B 206, 364 (1988).

6. P. Hagler, Phys. Rep. 490, 49 (2010) arXiv:0912.5483.

7. The RHIC SPIN Program, http://www.bnl.gov/npp/ docs/RHIC-Spin-WriteUp-121105.pdf.

8. A.V. Belitsky, X.-D. Ji, F. Yuan, Phys. Rev. D 69, 074014 (2004) arXiv:hep-ph/0307383.

9. M. Hillery, R. O'Connell, M. Scully, E.P. Wigner, Phys. Rep. 106, 121 (1984).

10. S. Glazov, Conf. Proc. C 0908171, 37 (2009).

11. F. Kunne, AIP Conf. Proc. 1560, 490 (2013).

12. Y.L. Dokshitzer, Sov. Phys. JETP 46, 641 (1977).

13. V. Gribov, L. Lipatov, Sov. J. Nucl. Phys. 15, 438 (1972).

14. G. Altarelli, G. Parisi, Nucl. Phys. B 126, 298 (1977).

15. E. Zijlstra, W. van Neerven, Nucl. Phys. B 417, 61 (1994).

16. R. Mertig, W. van Neerven, Z. Phys. C 70, 637 (1996) arXiv:hep-ph/9506451.

17. W. Vogelsang, Phys. Rev. D 54, 2023 (1996) arXiv:hep$\mathrm{ph} / 9512218$.
18. A. Vogt, S. Moch, M. Rogal, J. Vermaseren, Nucl. Phys. Proc. Suppl. 183, 155 (2008) arXiv:0807.1238.

19. R.L. Jaffe, A. Manohar, Nucl. Phys. B 337, 509 (1990).

20. X.-D. Ji, Phys. Rev. Lett. 78, 610 (1997) arXiv:hepph/9603249.

21. M. Wakamatsu, Phys. Rev. D 81, 114010 (2010) arXiv:1004.0268.

22. X. Ji, X. Xiong, F. Yuan, Phys. Rev. Lett. 109, 152005 (2012) arXiv:1202.2843.

23. M. Wakamatsu, Nuovo Cimento C 035, 247 (2012).

24. European Muon Collaboration (J. Ashman et al.), Nucl. Phys. B 328, 1 (1989).

25. F. Close, R. Roberts, Phys. Rev. Lett. 60, 1471 (1988).

26. F. Close, R. Roberts, Phys. Lett. B 316, 165 (1993) arXiv:hep-ph/9306289.

27. Spin Muon Collaboration (B. Adeva et al.), Phys. Rev. D 58, 112001 (1998).

28. COMPASS Collaboration (V. Alexakhin et al.), Phys. Lett. B 647, 8 (2007) arXiv:hep-ex/0609038.

29. COMPASS Collaboration (M.G. Alekseev et al.), Phys. Lett. B 690, 466 (2010) arXiv:1001.4654.

30. HERMES Collaboration (K. Ackerstaff et al.), Phys. Lett. B 404, 383 (1997) arXiv:hep-ex/9703005.

31. HERMES Collaboration (A. Airapetian et al.), Phys. Rev. D 75, 012007 (2007) arXiv:hep-ex/0609039.

32. Jefferson Lab Hall A Collaboration (X. Zheng et al.), Phys. Rev. C 70, 065207 (2004) arXiv:nucl-ex/0405006.

33. CLAS Collaboration (K. Dharmawardane et al.), Phys. Lett. B 641, 11 (2006) arXiv:nucl-ex/0605028.

34. E142 Collaboration (P. Anthony et al.), Phys. Rev. D 54, 6620 (1996) arXiv:hep-ex/9610007.

35. E143 Collaboration (K. Abe et al.), Phys. Rev. D 58, 112003 (1998) arXiv:hep-ph/9802357.

36. E154 Collaboration (K. Abe et al.), Phys. Rev. Lett. 79, 26 (1997) arXiv:hep-ex/9705012.

37. E155 Collaboration (P. Anthony et al.), Phys. Lett. B 463, 339 (1999) arXiv:hep-ex/9904002.

38. E155 Collaboration (P. Anthony et al.), Phys. Lett. B 493, 19 (2000) arXiv:hep-ph/0007248.

39. M. Hirai, S. Kumano, T.-H. Nagai, K. Sudoh, Phys. Rev. D 75, 094009 (2007) arXiv:hep-ph/0702250.

40. D. de Florian, R. Sassot, M. Stratmann, Phys. Rev. D 76, 074033 (2007) arXiv:0707.1506.

41. D. de Florian, R. Sassot, M. Stratmann, Phys. Rev. D 75, 114010 (2007) arXiv:hep-ph/0703242.

42. S. Albino, B. Kniehl, G. Kramer, Nucl. Phys. B 803, 42 (2008) arXiv:0803.2768.

43. D. de Florian et al., arXiv:1410.6027 (2014).

44. Spin Muon Collaboration (B. Adeva et al.), Phys. Lett. B 420, 180 (1998) arXiv:hep-ex/9711008.

45. COMPASS Collaboration (M. Alekseev et al.), Phys. Lett. B 660, 458 (2008) arXiv:0707.4077.

46. COMPASS Collaboration (M. Alekseev et al.), Phys. Lett. B 693, 227 (2010) arXiv:1007.4061.

47. HERMES Collaboration (A. Airapetian et al.), Phys. Rev. D 71, 012003 (2005) arXiv:hep-ex/0407032.

48. PHENIX Collaboration (S. Adler et al.), Phys. Rev. Lett. 93, 202002 (2004) arXiv:hep-ex/0404027.

49. PHENIX Collaboration (A. Adare et al.), Phys. Rev. D 76, 051106 (2007) arXiv:0704.3599.

50. PHENIX Collaboration (A. Adare et al.), Phys. Rev. Lett. 103, 012003 (2009) arXiv:0810.0694. 
51. STAR Collaboration (B. Abelev et al.), Phys. Rev. D 80, 111108 (2009) arXiv:0911.2773.

52. PHENIX Collaboration (A. Adare et al.), Phys. Rev. D 90, 012007 (2014) arXiv:1402.6296.

53. STAR Collaboration (B. Abelev et al.), Phys. Rev. Lett. 97, 252001 (2006) arXiv:hep-ex/0608030.

54. STAR Collaboration (B. Abelev et al.), Phys. Rev. Lett. 100, 232003 (2008) arXiv:0710.2048.

55. STAR Collaboration (M. Sarsour), AIP Conf. Proc. 1149, 389 (2009) arXiv:0901.4061.

56. STAR Collaboration (P. Djawotho), J. Phys. Conf. Ser. 295, 012061 (2011).

57. STAR Collaboration (L. Adamczyk et al.), arXiv:1405. 5134 (2014).

58. PHENIX Collaboration (A. Adare et al.), Phys. Rev. Lett. 106, 062001 (2011) arXiv:1009.0505.

59. STAR Collaboration (M. Aggarwal et al.), Phys. Rev. Lett. 106, 062002 (2011) arXiv:1009.0326.

60. STAR Collaboration (L. Adamczyk et al.), Phys. Rev. Lett. 113, 072301 (2014) arXiv:1404.6880.

61. PHENIX Collaboration (C. Gal), Phys. Part. Nucl. 45, 76 (2014)

62. D. de Florian, R. Sassot, M. Stratmann, W. Vogelsang, Phys. Rev. Lett. 101, 072001 (2008) arXiv:0804.0422.

63. D. de Florian, R. Sassot, M. Stratmann, W. Vogelsang, Phys. Rev. D 80, 034030 (2009) arXiv:0904.3821.

64. D. de Florian, R. Sassot, M. Stratmann, W. Vogelsang, Phys. Rev. Lett. 113, 012001 (2014) arXiv:1404.4293.

65. NNPDF Collaboration (E.R. Nocera et al.), Nucl. Phys. B 887, 276 (2014) arXiv:1406.5539.

66. G. Bunce, N. Saito, J. Soffer, W. Vogelsang, Annu. Rev. Nucl. Part. Sci. 50, 525 (2000) arXiv:hep-ph/0007218.

67. S. Kumano, Phys. Rep. 303, 183 (1998).

68. D. de Florian, W. Vogelsang, Phys. Rev. D 81, 094020 (2010) arXiv:1003.4533.

69. N. Cabibbo, E.C. Swallow, R. Winston, Annu. Rev. Nucl. Part. Sci. 53, 39 (2003) arXiv:hep-ph/0307298.

70. M.J. Savage, J. Walden, Phys. Rev. D 55, 5376 (1997) arXiv:hep-ph/9611210.

71. S.-L. Zhu, G. Sacco, M. Ramsey-Musolf, Phys. Rev. D 66, 034021 (2002) arXiv:hep-ph/0201179.

72. P.G. Ratcliffe, Czech. J. Phys. 54, B11 (2004) arXiv:hep$\mathrm{ph} / 0402063$.

73. S. Sasaki, T. Yamazaki, Phys. Rev. D 79, 074508 (2009) arXiv:0811.1406.

74. E. Leader, A.V. Sidorov, D.B. Stamenov, Phys. Rev. D 84, 014002 (2011) arXiv:1103.5979.

75. E.C. Aschenauer, R. Sassot, M. Stratmann, Phys. Rev. D 86, 054020 (2012) arXiv:1206.6014.

76. L. Mankiewicz, A. Schafer, M. Veltri, Comput. Phys. Commun. 71, 305 (1992).

77. J. Bjorken, Phys. Rev. 179, 1547 (1969).

78. E.C. Aschenauer et al., Phys. Rev. D 88, 114025 (2013) arXiv:1309.5327.

79. M. Anselmino et al., J. Phys. Conf. Ser. 295, 012062 (2011) arXiv:1012.3565.

80. HERMES Collaboration (A. Airapetian et al.), Phys. Rev. Lett. 94, 012002 (2005) arXiv:hep-ex/0408013.

81. COMPASS Collaboration (M. Alekseev et al.), Phys. Lett. B 673, 127 (2009) arXiv:0802.2160.

82. Jefferson Lab Hall A Collaboration (X. Qian et al.), Phys. Rev. Lett. 107, 072003 (2011) arXiv:1106.0363.
83. S.J. Brodsky, D.S. Hwang, I. Schmidt, Phys. Lett. B 530, 99 (2002).

84. J.C. Collins, Phys. Lett. B 536, 43 (2002).

85. A.V. Belitsky, X. Ji, F. Yuan, Nucl. Phys. B 656, 165 (2003).

86. D. Boer, P.J. Mulders, F. Pijlman, Nucl. Phys. B 667, 201 (2003) arXiv:hep-ph/0303034.

87. S.M. Aybat, J.C. Collins, J.-W. Qiu, T.C. Rogers, Phys. Rev. D 85, 034043 (2012) arXiv:1110.6428.

88. S.M. Aybat, A. Prokudin, T.C. Rogers, Phys. Rev. Lett. 108, 242003 (2012) arXiv:1112.4423.

89. P. Sun, F. Yuan, Phys. Rev. D 88, 034016 (2013) arXiv:1304.5037.

90. P. Sun, F. Yuan, Phys. Rev. D 88, 114012 (2013) arXiv:1308.5003.

91. M.G. Echevarria, A. Idilbi, Z.-B. Kang, I. Vitev, Phys. Rev. D 89, 074013 (2014) arXiv:1401.5078.

92. P. Su, J. Isaacson, C.P. Yuan, F. Yuan, arXiv:1406.3073 (2014).

93. X. Ji, J.-W. Qiu, W. Vogelsang, F. Yuan, Phys. Rev. Lett. 97, 082002 (2006).

94. A. Bacchetta, D. Boer, M. Diehl, P.J. Mulders, JHEP 08, 023 (2008).

95. S. Meissner, A. Metz, K. Goeke, Phys. Rev. D 76, 034002 (2007) arXiv:hep-ph/0703176.

96. T. Burton, arXiv:1212.3590 (2012).

97. J. Arrington, K. de Jager, C.F. Perdrisat, J. Phys. Conf. Ser. 299, 012002 (2011) arXiv:1102.2463.

98. M. Strikman, C. Weiss, Phys. Rev. D 69, 054012 (2004) arXiv:hep-ph/0308191.

99. M. Strikman, C. Weiss, Phys. Rev. D 80, 114029 (2009) arXiv:0906.3267.

100. M. Burkardt, Int. J. Mod. Phys. A 18, 173 (2003) arXiv:hep-ph/0207047.

101. M. Diehl, Eur. Phys. J. C 25, 223 (2002) arXiv:hep$\mathrm{ph} / 0205208$.

102. M. Burkardt, Nucl. Phys. A 735, 185 (2004) arXiv:hep$\mathrm{ph} / 0302144$.

103. L. Gamberg, M. Schlegel, AIP Conf. Proc. 1374, 309 (2011) arXiv:1012.3395.

104. M. Burkardt, G. Schnell, Phys. Rev. D 74, 013002 (2006) arXiv:hep-ph/0510249.

105. X.-D. Ji, J. Phys. G 24, 1181 (1998) arXiv:hep-ph/ 9807358.

106. M. Burkardt, Phys. Rev. D 72, 094020 (2005) arXiv:hep$\mathrm{ph} / 0505189$.

107. J.C. Collins, L. Frankfurt, M. Strikman, Phys. Rev. D 56, 2982 (1997).

108. J.C. Collins, A. Freund, Phys. Rev. D 59, 074009 (1999).

109. K. Kumericki, D. Mueller, K. Passek-Kumericki, Nucl. Phys. B 794, 244 (2008) arXiv:hep-ph/0703179.

110. B. Pire, L. Szymanowski, J. Wagner, Phys. Rev. D 83 , 034009 (2011) arXiv:1101.0555.

111. H. Moutarde et al., Phys. Rev. D 87, 054029 (2013) arXiv:1301.3819.

112. A. Belitsky, A. Radyushkin, Phys. Rep. 418, 1 (2005) arXiv:hep-ph/0504030.

113. V. Braun, A. Manashov, JHEP 01, 085 (2012) arXiv:1111.6765.

114. V. Braun, A. Manashov, B. Pirnay, Phys. Rev. Lett. 109, 242001 (2012) arXiv:1209.2559.

115. V.M. Braun, A.N. Manashov, D. Mueller, B.M. Pirnay, Phys. Rev. D 89, 074022 (2014) arXiv:1401.7621. 
116. A.V. Belitsky, D. Mueller, A. Kirchner, Nucl. Phys. B 629, 323 (2002).

117. A.V. Belitsky, D. Mueller, Y. Ji, Nucl. Phys. B 878, 214 (2014) arXiv:1212.6674.

118. A. Goritschnig, B. Pire, J. Wagner, arXiv:1404.0713 (2014).

119. P. Kroll, H. Moutarde, F. Sabatie, Eur. Phys. J. C 73, 2278 (2013) arXiv:1210.6975.

120. D. Ivanov, A. Schafer, L. Szymanowski, G. Krasnikov, Eur. Phys. J. C 34, 297 (2004) arXiv:hep-ph/0401131.

121. M. Diehl, W. Kugler, Eur. Phys. J. C 52, 933 (2007) arXiv:0708.1121.

122. S. Goloskokov, P. Kroll, Eur. Phys. J. C 50, 829 (2007) arXiv:hep-ph/0611290.

123. M. Diehl, W. Kugler, A. Schäfer, C. Weiss, Phys. Rev. D 72, 034034 (2005) arXiv:hep-ph/0506171.

124. S. Goloskokov, P. Kroll, Eur. Phys. J. C 53, 367 (2008) arXiv:0708.3569.

125. S. Ahmad, G.R. Goldstein, S. Liuti, Phys. Rev. D 79, 054014 (2009) arXiv:0805.3568.

126. S. Goloskokov, P. Kroll, Eur. Phys. J. A 47, 112 (2011) arXiv:1106.4897.

127. N. Warkentin, M. Diehl, D. Ivanov, A. Schäfer, Eur. Phys. J. A 32, 273 (2007) arXiv:hep-ph/0703148.

128. D.Y. Ivanov, B. Pire, L. Szymanowski, O. Teryaev, Phys. Lett. B 550, 65 (2002) arXiv:hep-ph/0209300.

129. D. Amrath, M. Diehl, J.-P. Lansberg, Eur. Phys. J. C 58, 179 (2008) arXiv:0807.4474

130. L. Frankfurt, M. Strikman, C. Weiss, Phys. Rev. D 69, 114010 (2004) arXiv:hep-ph/0311231.

131. M. Diehl, D. Ostermeier, A. Schäfer, JHEP 03, 089 (2012) arXiv:1111.0910.

132. K. Kumericki, D. Mueller, Nucl. Phys. B 841, 1 (2010) arXiv:0904.0458.

133. M. Diehl, Proceedings of DIS 2012, arXiv:1206.0844.

134. S. Fazio, arXiv:1212.3584 (2012).

135. E.-C. Aschenauer, S. Fazio, K. Kumericki, D. Mueller, JHEP 09, 093 (2013) arXiv:1304.0077.

136. J. Bartels, H. Kowalski, Eur. Phys. J. C 19, 693 (2001) arXiv:hep-ph/0010345.

137. K. Kumerički, D. Müller, Proceedings of DIS 2012, arXiv:1205.6967.

138. A. Bacchetta, U. D'Alesio, M. Diehl, C.A. Miller, Phys. Rev. D 70, 117504 (2004) arXiv:hep-ph/0410050.

139. J. Koempel, P. Kroll, A. Metz, J. Zhou, Phys. Rev. D 85. 051502 (2012) arXiv:1112.1334.

140. A. Malki et al., Phys. Rev. C 65, 015207 (2002) arXiv:nucl-ex/0005006.

141. R. Subedi et al., Science 320, 1476 (2008) arXiv:0908.1514.

142. L. Frankfurt, M. Sargsian, M. Strikman, Int. J. Mod. Phys. A 23, 2991 (2008) arXiv:0806.4412.

143. D.J. Gross, F. Wilczek, Phys. Rev. Lett. 30, 1343 (1973).

144. H.D. Politzer, Phys. Rev. Lett. 30, 1346 (1973).

145. E. Iancu, A. Leonidov, L.D. McLerran, Phys. Lett. B 510, 133 (2001).

146. I.I. Balitsky, L.N. Lipatov, Sov. J. Nucl. Phys. 28, 822 (1978).

147. E. Kuraev, L. Lipatov, V.S. Fadin, Sov. Phys. JETP 45, 199 (1977).

148. M. Froissart, Phys. Rev. 123, 1053 (1961).

149. I. Balitsky, Nucl. Phys. B 463, 99 (1996) arXiv:hepph/9509348.
150. Y.V. Kovchegov, Phys. Rev. D 60, 034008 (1999).

151. Y.V. Kovchegov, Phys. Rev. D 61, 074018 (2000).

152. L.V. Gribov, E.M. Levin, M.G. Ryskin, Phys. Rep. 100 1 (1983).

153. A.H. Mueller, J.-W. Qiu, Nucl. Phys. B 268, 427 (1986).

154. J. Jalilian-Marian, A. Kovner, A. Leonidov, H. Weigert, Phys. Rev. D 59, 014014 (1998).

155. J. Jalilian-Marian, A. Kovner, A. Leonidov, H. Weigert, Phys. Rev. D 59, 034007 (1999).

156. J. Jalilian-Marian, A. Kovner, H. Weigert, Phys. Rev. D 59, 014015 (1999).

157. E. Iancu, A. Leonidov, L.D. McLerran, Nucl. Phys. A 692, 583 (2001).

158. E. Iancu, K. Itakura, L. McLerran, Nucl. Phys. A 708, 327 (2002).

159. A.H. Mueller, D.N. Triantafyllopoulos, Nucl. Phys. B 640, 331 (2002).

160. L.D. McLerran, R. Venugopalan, Phys. Rev. D 49, 2233 (1994).

161. Y.V. Kovchegov, Phys. Rev. D 54, 5463 (1996).

162. J. Jalilian-Marian, A. Kovner, L.D. McLerran, H. Weigert, Phys. Rev. D 55, 5414 (1997).

163. A.H. Mueller, Nucl. Phys. B 335, 115 (1990).

164. J.L. Albacete, Phys. Rev. Lett. 99, 262301 (2007).

165. J.L. Albacete, N. Armesto, J.G. Milhano, C.A. Salgado, Phys. Rev. D 80, 034031 (2009) arXiv:0902.1112.

166. J.L. Albacete et al., Eur. Phys. J. C 71, 1705 (2011) arXiv:1012.4408.

167. K. Golec-Biernat, M. Wüsthoff, Phys. Rev. D 59, 014017 (1999).

168. K.J. Golec-Biernat, M. Wusthoff, Phys. Rev. D 60 , 114023 (1999).

169. J. Jalilian-Marian, Y.V. Kovchegov, Prog. Part. Nucl. Phys. 56, 104 (2006).

170. H. Weigert, Prog. Part. Nucl. Phys. 55, 461 (2005) arXiv:hep-ph/0501087.

171. E. Iancu, R. Venugopalan, arXiv:hep-ph/0303204 (2003).

172. F. Gelis, E. Iancu, J. Jalilian-Marian, R. Venugopalan, Annu. Rev. Nucl. Part. Sci. 60, 463 (2010) arXiv:1002.0333.

173. Y.V. Kovchegov, E. Levin, Quantum Chromodynamics at High Energy (Cambridge University Press, 2012).

174. H. Kowalski, D. Teaney, Phys. Rev. D 68, 114005 (2003).

175. N.N. Nikolaev, B. Zakharov, Phys. Lett. B 332, 184 (1994) arXiv:hep-ph/9403243.

176. K. Eskola, H. Paukkunen, C. Salgado, JHEP 04, 065 (2009) arXiv:0902.4154.

177. K.J. Eskola, V.J. Kolhinen, C.A. Salgado, Eur. Phys. J. C 9, 61 (1999).

178. M. Hirai, S. Kumano, T.-H. Nagai, Phys. Rev. C $\mathbf{7 6}$ 065207 (2007) arXiv:0709.3038.

179. D. de Florian, R. Sassot, Phys. Rev. D 69, 074028 (2004) arXiv:hep-ph/0311227.

180. I. Balitsky, Phys. Rev. D 75, 014001 (2007) arXiv:hepph/0609105.

181. Y.V. Kovchegov, H. Weigert, Nucl. Phys. A 784, 188 (2007).

182. E. Gardi, J. Kuokkanen, K. Rummukainen, H. Weigert, Nucl. Phys. A 784, 282 (2007).

183. J.L. Albacete, Y.V. Kovchegov, Phys. Rev. D 75, 125021 (2007).

184. L.L. Frankfurt, M.I. Strikman, Phys. Rep. 160, 235 (1988). 
185. A.P. Bukhvostov, G.V. Frolov, L.N. Lipatov, E.A. Kuraev, Nucl. Phys. B 258, 601 (1985).

186. J. Bartels, K. Golec-Biernat, L. Motyka, Phys. Rev. D 81, 054017 (2010) arXiv:0911.1935.

187. K.J. Golec-Biernat, M. Wusthoff, Phys. Rev. D 59, 014017 (1998) arXiv:hep-ph/9807513.

188. S. Munier, A.M. Stasto, A.H. Mueller, Nucl. Phys. B 603, 427 (2001).

189. H. Abramowicz, A. Caldwell, Rev. Mod. Phys. 71, 1275 (1999) arXiv:hep-ex/9903037.

190. A. Martin, W. Stirling, R. Thorne, G. Watt, Eur. Phys. J. C 63, 189 (2009) arXiv:0901.0002.

191. D. Kharzeev, E. Levin, L. McLerran, Nucl. Phys. A 748, 627 (2005).

192. F. Dominguez, C. Marquet, B.-W. Xiao, F. Yuan, Phys. Rev. D 83, 105005 (2011) arXiv:1101.0715.

193. F. Dominguez, B.-W. Xiao, F. Yuan, Phys. Rev. Lett. 106, 022301 (2011).

194. L. Zheng, E. Aschenauer, J. Lee, B.-W. Xiao, Phys. Rev. D 89, 074037 (2014) arXiv:1403.2413.

195. PHENIX Collaboration (A. Adare et al.), Phys. Rev. Lett. 107, 172301 (2011) arXiv:1105.5112.

196. STAR Collaboration (E. Braidot), Nucl. Phys. A 854, 168 (2011) arXiv:1008.3989.

197. T. Sjostrand, S. Mrenna, P.Z. Skands, JHEP 05, 026 (2006) arXiv:hep-ph/0603175.

198. S. Roesler, R. Engel, J. Ranft, arXiv:hep-ph/0012252 (2000) p. 1033.

199. C.A. Salgado, U.A. Wiedemann, Phys. Rev. D 68, 014008 (2003) arXiv:hep-ph/0302184.

200. B. Kopeliovich, Phys. Rev. C 68, 044906 (2003) arXiv:nucl-th/0306044.

201. H. Kowalski, T. Lappi, C. Marquet, R. Venugopalan, Phys. Rev. C 78, 045201 (2008) arXiv:0805.4071.

202. H. Kowalski, T. Lappi, R. Venugopalan, Phys. Rev. Lett. 100, 022303 (2008).

203. T. Toll, T. Ullrich, Phys. Rev. C 87, 024913 (2013) arXiv:1211.3048.

204. T. Toll, T. Ullrich, arXiv:1307.8059 (2013).

205. W. Buchmuller, M. McDermott, A. Hebecker, Nucl. Phys. B 487, 283 (1997) arXiv:hep-ph/9607290.

206. Y.V. Kovchegov, L.D. McLerran, Phys. Rev. D 60, 054025 (1999).

207. L. Frankfurt, V. Guzey, M. Strikman, Phys. Lett. B 586, 41 (2004).

208. L. Frankfurt, V. Guzey, M. Strikman, Phys. Rep. 512, 255 (2012) arXiv:1106.2091.

209. ZEUS Collaboration (J. Breitweg et al.), Eur. Phys. J. C 6, 43 (1999) arXiv:hep-ex/9807010.

210. H. Kowalski, L. Motyka, G. Watt, Phys. Rev. D 74, 074016 (2006).

211. F. Aaron et al., Eur. Phys. J. C 72, 1836 (2012).

212. CLAS Collaboration (W. Brooks et al.), AIP Conf. Proc. 1441, 211 (2012) arXiv:1110.3268.

213. European Muon Collaboration (J.J. Aubert et al.), Phys. Lett. B 123, 275 (1983).

214. S.J. Brodsky, H.J. Lu, Phys. Rev. Lett. 64, 1342 (1990).

215. A.H. Mueller, J.-W. Qiu, Nucl. Phys. B 268, 427 (1986).

216. J.-W. Qiu, I. Vitev, Phys. Rev. Lett. 93, 262301 (2004) arXiv:hep-ph/0309094.

217. D. d'Enterria, arXiv:0902.2011 (2009).

218. X.-N. Wang, M. Gyulassy, Phys. Rev. Lett. 68, 1480 (1992).
219. T. Kneesch, B. Kniehl, G. Kramer, I. Schienbein, Nucl. Phys. B 799, 34 (2008) arXiv:0712.0481.

220. X.-F. Guo, X.-N. Wang,, Phys. Rev. Lett. 85, 3591 (2000).

221. PHENIX Collaboration (S. Tarafdar), arXiv:1208.0456 (2012).

222. B.Z. Kopeliovich, J. Nemchik, E. Predazzi, A. Hayashigaki, Nucl. Phys. A 740, 211 (2004).

223. A. Accardi, D. Grunewald, V. Muccifora, H.J. Pirner, Nucl. Phys. A 761, 67 (2005).

224. G. Bertsch, S.J. Brodsky, A. Goldhaber, J. Gunion, Phys. Rev. Lett. 47, 297 (1981).

225. S.J. Brodsky, A.H. Mueller, Phys. Lett. B 206, 685 (1988).

226. L. Frankfurt, G. Miller, M. Strikman, Annu. Rev. Nucl. Part. Sci. 44, 501 (1994) arXiv:hep-ph/9407274.

227. D. Dutta, K. Hafidi, M. Strikman, arXiv:1211.2826 (2012).

228. X. Guo, J. Li, arXiv:0705.4211 (2007).

229. E. Wang, X.-N. Wang, Phys. Rev. Lett. 89, 162301 (2002).

230. B.-W. Zhang, E. Wang, X.-N. Wang, Nucl. Phys. A 757, 493 (2005) arXiv:hep-ph/0412060.

231. J. Raufeisen, Phys. Lett. B 557, 184 (2003) arXiv:hep$\mathrm{ph} / 0301052$.

232. T. Liou, A. Mueller, B. Wu, Nucl. Phys. A 916, 102 (2013) arXiv:1304.7677.

233. D. Pitonyak, J.-W. Qiu, in preparation.

234. F. Caola, S. Forte, J. Rojo, Nucl. Phys. A 854, 32 (2011) arXiv:1007.5405.

235. BRAHMS Collaboration (I. Arsene et al.), Phys. Rev. Lett. 93, 242303 (2004) arXiv:nucl-ex/0403005.

236. PHENIX Collaboration (S.S. Adler et al.), Phys. Rev. Lett. 98, 172302 (2007).

237. STAR Collaboration (J. Adams et al.), Phys. Rev. Lett. 97, 152302 (2006) arXiv:nucl-ex/0602011.

238. ALICE Collaboration (B. Abelev et al.), Phys. Rev. Lett. 110, 082302 (2013) arXiv:1210.4520.

239. A. Dumitru, A. Hayashigaki, J. Jalilian-Marian, Nucl. Phys. A 765, 464 (2006) arXiv:hep-ph/0506308.

240. J.L. Albacete, C. Marquet, Nucl. Phys. A 854, 154 (2011) arXiv:1009.3215.

241. D. Kharzeev, E. Levin, L. McLerran, Phys. Lett. B 561, 93 (2003) arXiv:hep-ph/0210332.

242. J.L. Albacete et al., Phys. Rev. D 71, 014003 (2005).

243. D. Kharzeev, Y.V. Kovchegov, K. Tuchin, Phys. Rev. D 68, 094013 (2003).

244. D. Kharzeev, Y.V. Kovchegov, K. Tuchin, Phys. Lett. B 599, 23 (2004)

245. V. Guzey, M. Strikman, W. Vogelsang, Phys. Lett. B 603 , 173 (2004) arXiv:hep-ph/0407201.

246. B. Kopeliovich et al., Phys. Rev. C 72, 054606 (2005) arXiv:hep-ph/0501260.

247. C. Marquet, Nucl. Phys. A 796, 41 (2007).

248. J.L. Albacete, C. Marquet, Phys. Rev. Lett. 105, 162301 (2010).

249. STAR Collaboration (J. Adams et al.), Phys. Rev. Lett. 95, 152301 (2005) arXiv:nucl-ex/0501016.

250. PHENIX Collaboration (A. Adare et al.), Phys. Rev. C 78, 014901 (2008) arXiv:0801.4545. 
251. PHOBOS Collaboration (B. Alver et al.), Phys. Rev. Lett. 104, 062301 (2010) arXiv:0903.2811.

252. STAR Collaboration (B. Abelev et al.), Phys. Rev. C 80, 064912 (2009) arXiv:0909.0191.

253. CMS Collaboration (V. Khachatryan et al.), JHEP 09, 091 (2010) arXiv:1009.4122.

254. CMS Collaboration (S. Chatrchyan et al.), Phys. Lett. B 718, 795 (2013) arXiv:1210.5482.

255. ALICE Collaboration (B. Abelev et al.), Phys. Lett. B 719, 29 (2013) arXiv:1212.2001.

256. CMS Collaboration (S. Chatrchyan et al.), Phys. Lett. B 724, 213 (2013) arXiv:1305.0609.

257. A. Dumitru, F. Gelis, L. McLerran, R. Venugopalan, Nucl. Phys. A 810, 91 (2008) arXiv:0804.3858.

258. A. Dumitru et al., Phys. Lett. B 697, 21 (2011) arXiv:1009.5295.

259. K. Dusling, R. Venugopalan, Phys. Rev. D 87, 054014 (2013) arXiv:1211.3701

260. A. Kovner, M. Lublinsky, Int. J. Mod. Phys. E 22, 1330001 (2013) arXiv:1211.1928.

261. Y.V. Kovchegov, D.E. Wertepny, Nucl. Phys. A 906, 50 (2013) arXiv:1212.1195.

262. K. Dusling, R. Venugopalan, Phys. Rev. D 87, 094034 (2013) arXiv:1302.7018.

263. ALICE Collaboration (B.B. Abelev et al.), arXiv:1404. 1194 (2014).

264. B.Z. Kopeliovich, A.V. Tarasov, A. Schafer, Phys. Rev. C 59, 1609 (1999) arXiv:hep-ph/9808378.

265. A. Accardi et al., arXiv:hep-ph/0308248 (2004).

266. PHENIX Collaboration (K. Adcox et al.), Nucl. Phys. A 757, 184 (2005).

267. STAR Collaboration (J. Adams et al.), Nucl. Phys. A 757, 102 (2005)

268. PHOBOS Collaboration (B.B. Back et al.), Nucl. Phys. A 757, 28 (2005).

269. BRAHMS Collaboration (I. Arsene et al.), Nucl. Phys. A 757, 1 (2005).

270. M. Gyulassy, L. McLerran, Nucl. Phys. A 750, 30 (2005).

271. I. Tserruya, AIP Conf. Proc. 1422, 166 (2012) arXiv: 1110.4047 .

272. J.M. Maldacena, Adv. Theor. Math. Phys. 2, 231 (1998) hep-th/9711200.

273. O. Aharony et al., Phys. Rep. 323, 183 (2000) hepth/9905111.

274. P.F. Kolb, U.W. Heinz, arXiv:nucl-th/0305084 (2003).

275. D. Teaney, J. Lauret, E.V. Shuryak, Phys. Rev. Lett. 86, 4783 (2001) arXiv:nucl-th/0011058.

276. M. Luzum, P. Romatschke, Phys. Rev. C 79, 039903 (2009).

277. A. Kovner, L.D. McLerran, H. Weigert, Phys. Rev. D 52, 6231 (1995) arXiv:hep-ph/9502289.

278. A. Krasnitz, R. Venugopalan, Nucl. Phys. B 557, 237 (1999) arXiv:hep-ph/9809433.

279. S. Mrowczynski, Phys. Lett. B 314, 118 (1993).

280. P.B. Arnold, J. Lenaghan, G.D. Moore, JHEP 08, 002 (2003) arXiv:hep-ph/0307325.

281. Y.V. Kovchegov, Rept. Prog. Phys. 75, 124301 (2012) arXiv:1112.5403.

282. P.M. Chesler, L.G. Yaffe, Phys. Rev. Lett. 106, 021601 (2011) arXiv:1011.3562.

283. J. Bartels, K.J. Golec-Biernat, H. Kowalski, Phys. Rev. D 66, 014001 (2002).

284. J.L. Albacete, A. Dumitru, arXiv:1011.5161 (2010).
285. PHOBOS Collaboration (B.B. Back et al.), Phys. Rev. C 65, 061901 (2002).

286. The ALICE Collaboration (B. Abelev et al.), Phys. Rev. Lett. 105, 252301 (2010) arXiv:1011.3916.

287. B. Schenke, P. Tribedy, R. Venugopalan, Phys. Rev. C 86, 034908 (2012) arXiv:1206.6805.

288. B. Schenke, P. Tribedy, R. Venugopalan, Phys. Rev. Lett. 108, 252301 (2012) arXiv:1202.6646.

289. J.Y. Ollitrault, Phys. Rev. D 46, 229 (1992).

290. D. Kharzeev, E. Levin, M. Nardi, Nucl. Phys. A 743, 329 (2004).

291. G. Policastro, D.T. Son, A.O. Starinets, Phys. Rev. Lett. 87, 081601 (2001) hep-th/0104066.

292. P. Kovtun, D.T. Son, A.O. Starinets, Phys. Rev. Lett. 94, 111601 (2005) hep-th/0405231.

293. B. Schenke, S. Jeon, C. Gale, Phys. Rev. C 85, 024901 (2012) arXiv:1109.6289.

294. PHENIX Collaboration (A. Adare et al.), Phys. Rev. Lett. 107, 252301 (2011) arXiv:1105.3928.

295. C. Marquet, H. Weigert, Nucl. Phys. A 843, 68 (2010) arXiv:1003.0813.

296. HERMES Collaboration (A. Airapetian et al.), Nucl. Phys. B 780, 1 (2007).

297. W.K. Brooks, H. Hakobyan, Nucl. Phys. A 830, 361c (2009).

298. C.A. Bertulani, S.R. Klein, J. Nystrand, Annu. Rev. Nucl. Part. Sci. 55, 271 (2005) arXiv:nucl-ex/0502005.

299. ALICE Collaboration (E. Abbas et al.), Eur. Phys. J. C 73, 2617 (2013) arXiv:1305.1467.

300. V. Goncalves, M. Machado, J. Phys. G 32, 295 (2006) arXiv:hep-ph/0506331.

301. L.A. Anchordoqui, A.M. Cooper-Sarkar, D. Hooper, S. Sarkar, Phys. Rev. D 74, 043008 (2006) arXiv:hepph/0605086.

302. E.M. Henley, J. Jalilian-Marian, Phys. Rev. D 73, 094004 (2006) arXiv:hep-ph/0512220.

303. K. Kutak, J. Kwiecinski, Eur. Phys. J. C 29, 521 (2003) arXiv:hep-ph/0303209.

304. Pierre Auger Collaboration (P. Abreu et al.), arXiv:1107. 4804 (2011).

305. IceCube Collaboration (R. Abbasi et al.), arXiv:1208. 2979 (2012).

306. H1 Collaboration (C. Adloff et al.), Eur. Phys. J. C 11, 447 (1999) arXiv:hep-ex/9907002.

307. ZEUS Collaboration (S. Chekanov et al.), Eur. Phys. J. C 44, 463 (2005) arXiv:hep-ex/0501070.

308. H1 Collaboration (A. Aktas et al.), Eur. Phys. J. C 52 , 833 (2007) arXiv:hep-ex/0703004.

309. C.H. Albright, M.-C. Chen, Phys. Rev. D 77, 113010 (2008) arXiv:0802.4228.

310. W. Buchmuller, R. Ruckl, D. Wyler, Phys. Lett. B 191, 442 (1987).

311. M. Gonderinger, M.J. Ramsey-Musolf, JHEP 11, 045 (2010) arXiv:1006.5063.

312. ATLAS Collaboration (G. Aad et al.), Phys. Lett. B 716 , 1 (2012) arXiv:1207.7214.

313. CMS Collaboration (S. Chatrchyan et al.), Phys. Lett. B 716, 30 (2012) arXiv:1207.7235.

314. Qweak Collaboration (D. Androic et al.), Phys. Rev. Lett. 111, 141803 (2013) arXiv:1307.5275.

315. M. Ramsey-Musolf, Phys. Rev. C 60, 015501 (1999) arXiv:hep-ph/9903264. 
316. R.D. Young, R.D. Carlini, A.W. Thomas, J. Roche, Phys. Rev. Lett. 99, 122003 (2007) arXiv:0704.2618.

317. J. Erler, S. Su, Prog. Part. Nucl. Phys. 71, 119 (2013) arXiv:1303.5522.

318. V. Cirigliano, M.J. Ramsey-Musolf, Prog. Part. Nucl. Phys. 71, 2 (2013) arXiv:1304.0017.

319. K. Kumar, S. Mantry, W. Marciano, P. Souder, Annu. Rev. Nucl. Part. Sci. 63, 237 (2013) arXiv:1302.6263.

320. PVDIS Collaboration (D. Wang et al.), Nature 506, 67 (2014).

321. M.R. Buckley, M.J. Ramsey-Musolf, Phys. Lett. B 712, 261 (2012) arXiv:1203.1102.

322. E.C. Aschenauer et al., arXiv:1409.1633 (2014).

323. S. Abeyratne et al., arXiv:1504.07961 (2015).

324. Y. Zhang, J. Bisognano, arXiv:1209.0757 (2012).

325. Y. Derbenev, G. Krafft, B. Yunn, Y. Zhang, Proceedings of HB2010, Morschach, Switzerland (2010) (Paul Scherrer Institute, Villigen, Switzerland, 2011) p. 49.

326. Y. Derbenev, Y. Zhang, Proceedings of COOL09, Lanzhou, China (2009) (Inst. Mod. Phys., Lanzhou, P.R. China, 2010) p. 181.

327. Y. Derbenev, University of Michigan Report No. UM-HE96-05, 1996 (unpublished).

328. T. Clegg et al., Nucl. Instrum. Methods Phys. Res. A 357, 200 (1995).

329. J. Alessi et al., Proceedings of LINAC 2006, Knoxville, Tennessee, USA (2006) (ORNL, Oak Ridge, USA, 2006) p. 385 .

330. B. Mustapha, P. Ostroumov, J. Nolen, A driver linac for the advanced exotic beam laboratory: Physics design and beam dynamics simulations, in 22nd Particle Accelerator Conference (PAC 07) Vol. 1 (IEEE, Piscataway, USA, 2007) p. 1661.

331. V. Morozov, R. Ent, P. Nadel-Turonski, C. Hyde, Proceedings of IPAC'12, New Orleans, USA (2012) (IEEE, Piscataway, USA, 2012) p. 2011.

332. V. Morozov, Y.S. Derbenev, F. Lin, R. Johnson, Phys. Rev. ST 16, 011004 (2013).

333. Y.S. Derbenev et al., Proceedings of PSTP'13, Charlottesville, VA, USA, PoS(PSTP2013)026 (2013).
334. P. Chevtsov, Y. Derbenev, G. Krafft, Y. Zhang, Thomas Jefferson National Accelerator Facility Report No. JLabTN-10-026, 2010 (unpublished).

335. F. Lin et al., Proceedings of PSTP'13, Charlottesville, VA, USA, PoS(PSTP2013)025 (2013).

336. S. Nagaitsev et al., Phys. Rev. Lett. 96, 044801 (2006).

337. J. Dietrich et al., Proceedings of IPAC'10, Kyoto, Japan (2010) (JACoW, Geneva, Switzerland, 2010) p. 843.

338. F. Jacquet, A. Blondel, DESY 79/48, in Proceedings of the Study of an ep Facility for Europe, edited by U. Amaldi (DESY, Hamburg, Germany, 1979).

339. U. Bassler, G. Bernardi, Nucl. Instrum. Methods A 361 , 197 (1995) arXiv:hep-ex/9412004.

340. http://www.desy.de/pol2000/Welcome.html.

341. https://wiki.bnl.gov/rhicspin/Polarimetry.

342. C. Aidala et al., sPHENIX: An Upgrade Concept from the PHENIX Collaboration, arXiv:1207.6378 (2012).

343. M. Shao, Nucl. Instrum. Methods A 558, 419 (2006).

344. PHENIX Collaboration (A. Adare et al.), arXiv:1402. 1209 (2014).

345. eSTAR Letter of Intent, https://wiki.bnl.gov/ rhicspin/Polarimetry.

346. EicRoot simulation framework, http://svn.racf.bnl. gov/svn/eic/eicroot.

347. F. Sabatie, Progress report for the EIC Detector RED Committee, https://wiki.bnl.gov/conferences/ images/1/1a/EIC-RD-MIT-Saclay-Temple_v3.pdf (2013).

348. G. Gaycken et al., Nucl. Instrum. Methods A 560, 44 (2006).

349. C. Hu-Guo et al., JINST 4, P04012 (2009).

350. STAR Collaboration (J. Kapitan), Indian J. Phys. 85, 177 (2011) arXiv:0806.2266.

351. PANDA Collaboration, arXiv:0810.1216 (2008).

352. CMS Collaboration (Y. Musienko), Nucl. Instrum. Methods A 494, 308 (2002).

353. E.C. Aschenauer et al., REDD Proposal for an electron polarimeter, a luminosity monitor and a low Q2-tagger, https://wiki.bnl.gov/conferences/images/8/82/ RD2013-6_Aschenauer.pdf (2014). 